\title{
Rigidity and uniruling for Lagrangian submanifolds
}

\author{
PAUL BIRAN \\ OCTAV CORNEA
}

\begin{abstract}
This paper explores the topology of monotone Lagrangian submanifolds $L$ inside a symplectic manifold $M$ by exploiting the relationships between the quantum homology of $M$ and various quantum structures associated to the Lagrangian $L$.
\end{abstract}

53D12; 53D05

\section{Introduction}

The purpose of this paper is to explore the topology of monotone Lagrangian submanifolds $L$ inside a symplectic manifold $M$ by exploiting the relationships between the quantum homology of $M$ and various quantum structures associated to the Lagrangian $L$. We show that the class of monotone Lagrangians satisfies a number of structural rigidity properties which are particularly strong when the ambient symplectic manifold contains enough genus-zero pseudo-holomorphic curves. Indeed, we will see that (very often) if $M$ is "highly" uniruled by curves of area $A$, then $(M, L)$ (or just $L$ ) is uniruled by curves of area strictly smaller than $A$ (see Section 1.1.2 for the definition of the appropriate notions of uniruling).

\subsection{Setting}

All our symplectic manifolds will be implicitly assumed to be connected and tame (see Audin, Lalonde and Polterovich [3]). The main examples of such manifolds are closed symplectic manifolds, manifolds which are symplectically convex at infinity as well as products of such. All the Lagrangian submanifolds will be assumed to be connected and closed (ie compact, without boundary).

We start by emphasizing that our results apply to monotone Lagrangians. These are characterized by the fact that the morphisms

$$
\omega: \pi_{2}(M, L) \rightarrow \mathbb{R}, \quad \mu: \pi_{2}(M, L) \rightarrow \mathbb{Z},
$$


the first given by integration and the second by the Maslov index, are proportional with a positive proportionality constant $\omega=\eta \mu$ with $\eta>0$. Moreover, we will include here in the definition of the monotonicity the assumption that the minimal Maslov index

$$
N_{L}=\min \left\{\mu(\alpha) \mid \alpha \in \pi_{2}(M, L), \mu(\alpha)>0\right\}
$$

of a homotopy class of strictly positive Maslov index is at least two, $N_{L} \geq 2$. If $L$ is monotone, then $M$ is also monotone and $N_{L}$ divides $2 C_{M}$ where $C_{M}$ is the minimal Chern number of $M$

$$
C_{M}=\min \left\{c_{1}(\alpha) \mid \alpha \in \pi_{2}(M), c_{1}(\alpha)>0\right\} .
$$

\subsubsection{Size of Lagrangians Fix a Lagrangian submanifold $L \subset M$.}

We say that a symplectic embedding of the closed, standard symplectic ball of radius $r$, $e:\left(B^{2 n}(r), \omega_{\mathrm{std}}\right) \rightarrow(M, \omega)$, is relative to $L$ if

$$
e^{-1}(L)=B^{2 n}(r) \cap \mathbb{R}^{n} .
$$

These types of embeddings were first introduced and used by Barraud and Cornea $[4 ; 5]$.

Consider now a vector $v_{p, q}=\left(r_{1}, \ldots, r_{p} ; \rho_{1}, \ldots, \rho_{q}\right) \in\left(\mathbb{R}^{+}\right)^{p+q}$. We will not allow for both $p$ and $q$ to vanish. If just one does, say $p=0$, we will use the notation $v_{0, q}=\left(\varnothing ; \rho_{1}, \ldots, \rho_{q}\right)$.

Definition 1.1.1 The mixed symplectic packing number, $w\left(M, L: v_{p, q}\right)$, of type $v_{p, q}=\left(r_{1}, \ldots, r_{p} ; \rho_{1}, \ldots, \rho_{q}\right)$ of $(M, L)$ is defined by

$$
w\left(M, L: v_{p, q}\right)=\sup _{\tau>0}\left(\sum_{i=1}^{p} \pi\left(\tau r_{i}\right)^{2}+\frac{1}{2} \sum_{j=1}^{q} \pi\left(\tau \rho_{j}\right)^{2}\right)
$$

where the supremum is taken over all $\tau$ such that there are mutually disjoint symplectic embeddings

$$
f_{i}:\left(B^{2 n}\left(\tau r_{i}\right), \omega_{0}\right) \rightarrow(M \backslash L), 1 \leq i \leq p, \quad e_{j}:\left(B^{2 n}\left(\tau \rho_{j}\right), \omega_{0}\right) \rightarrow M, 1 \leq j \leq q
$$

so that the $e_{j}$ 's are embeddings relative to $L$.

The most widespread examples of such vectors $v_{p, q}$ have all their components equal to 1 . We also notice that $w(M):=w(M, \varnothing:(1 ; \varnothing))$ is the well-known Gromov width of $M$ : the supremum of $\pi r^{2}$ over all symplectic embeddings of $B^{2 n}(r)$ into $M$. A similar notion has been introduced by Barraud and Cornea [4] (see also Cornea and Lalonde [25]) to "measure" Lagrangians: the width of a Lagrangian, $w(L)$, is the 
supremum of $\pi r^{2}$ over all symplectic embeddings of $B^{2 n}(r)$ which are relative to $L$. With our conventions, $w(L)=2 w(M, L:(\varnothing ; 1))$. Moreover, $w(M \backslash L)$, the Gromov width of the complement of $L$, is given by $w(M, L:(1 ; \varnothing))$.

1.1.2 Uniruling The main technique used to prove width and packing estimates is based on establishing uniruling results.

Definition 1.1.2 We say that $(M, L)$ is uniruled of type $(p, q)$ and order $k$ (or shorter, $(M, L)$ is $(p, q)$-uniruled of order $k)$ if for any $p$ distinct points $P_{i} \in M \backslash L, 1 \leq i \leq p$, and any $q$ distinct points, $Q_{j} \in L, 1 \leq j \leq q$, there exists a Baire second category (generic) family of almost complex structures $\mathcal{J}$ with the property that for each $J \in \mathcal{J}$ there exists a nonconstant $J$-holomorphic disk $u:\left(D^{2}, \partial D^{2}\right) \rightarrow(M, L)$ so that $P_{i} \in u\left(\operatorname{Int}\left(D^{2}\right)\right)$ for all $i, Q_{j} \in u\left(\partial D^{2}\right)$ for all $j$, and $\mu(u) \leq k$. In case $L$ is void, we take $q=0$, and instead of a disk, $u$ is required to be a nonconstant $J$-holomorphic sphere so that $P_{i} \in u\left(S^{2}\right)$, for all $i$.

If $(M, \varnothing)$ is $(p, 0)$-uniruled we will say that $M$ is uniruled of type $p$. Thus the usual notion of uniruling for a symplectic manifold $-M$ is uniruled if through each point of $M$ passes a $J$-sphere in some fixed homotopy class in $\pi_{2}(M)$ - is equivalent in our terminology with $M$ being 1 -uniruled. Similarly, in case $(M, L)$ is $(0, q)$-uniruled we will say that $L$ is $q$-uniruled. Additionally, if $q=1$ we say that $L$ is uniruled.

The relation with packing is given by the following fact:

Lemma 1.1.3 If the pair $(M, L)$ is $(p, q)$-uniruled of order $k$, then for any vector $v_{p, q}=\left(r_{1}, \ldots r_{p} ; \rho_{1}, \ldots \rho_{q}\right)$ the mixed symplectic packing number $w\left(M, L: v_{p, q}\right)$ satisfies

$$
w\left(M, L: v_{p, q}\right) \leq \eta k
$$

where $\eta$ is the monotonicity constant, $\eta=\omega / \mu$.

The proof of this is standard and is a small modification of an argument of Gromov [35]. It comes down to the following simple remark which also explains the $1 / 2$ factor in the definition of $w\left(M, L: v_{p, q}\right)$. If a $J$-curve $u$ with boundary on a Lagrangian goes through the center of a standard symplectic ball or radius $r$ embedded in $M$ relative to $L$ so that $J$ coincides with the standard almost complex structure inside the ball, then we have $\pi r^{2} / 2 \leq \int u^{*} \omega$. This is in contrast to the case when $u$ has no boundary, when the inequality is, as is well-known, $\pi r^{2} \leq \int u^{*} \omega$.

The simplest way to detect algebraically that $M$ is $p$-uniruled is to find some class $\alpha \in \pi_{2}(M)$ and $r \geq 1$ so that, for distinct points $P_{1}, \ldots, P_{p}$, and a generic $J$, the 
evaluation at $r$ distinct points on the $J$-spheres of class $\alpha$ which pass through the fixed points $P_{i}, 1 \leq i \leq p$, has a homologically nontrivial image in the product $M^{\times r}$. This can be translated in terms of Gromov-Witten invariants: if there exist $\alpha \in \pi_{2}(M)$ and classes $a_{i} \in H_{*}\left(M ; \mathbb{Z}_{2}\right), 1 \leq i \leq r$, so that

$$
\mathrm{GW}\left(\mathrm{pt}, \ldots, \mathrm{pt}, a_{1}, \ldots, a_{r} ; \alpha\right) \neq 0
$$

where the class of the point, pt $\in H_{0}\left(M ; \mathbb{Z}_{2}\right)$, appears $p$ times, then $M$ is clearly $p$-uniruled (we recall that the Gromov-Witten invariant $\mathrm{GW}\left(b_{1}, \ldots b_{s} ; \alpha\right)$ counts in this paper with $\mathbb{Z}_{2}$ coefficients - the number of $J$-spheres in the homotopy class $\alpha \in \pi_{2}(M)$ which each pass through generic cycles representing the homology classes $\left.b_{i} \in H_{*}\left(M ; \mathbb{Z}_{2}\right)\right)$.

Remark 1.1.4 In case $p=1$ the condition in (1) gives the notion of "strong uniruled" which appears in McDuff [39] (with the additional constraint that the degree of the homology classes $a_{i}$ are even).

If we fix $p \geq 2$ and add the requirement that $r=1$, then, by the splitting property of Gromov-Witten invariants, the uniruling condition implies $\mathrm{GW}\left(\mathrm{pt}, \mathrm{pt}, a ; \alpha^{\prime}\right) \neq 0$ for some choices of $a \in H_{*}\left(M ; \mathbb{Z}_{2}\right)$ and $\alpha^{\prime} \in \pi_{2}(M)$. Of course, this can be reinterpreted in quantum homology as the relation $[\mathrm{pt}] * a=[M] e^{\alpha^{\prime}}+\cdots$ where $[\mathrm{pt}] \in H_{0}\left(M ; \mathbb{Z}_{2}\right)$ represents the point, $[M] \in H_{2 n}\left(M, \mathbb{Z}_{2}\right)$ is the fundamental class, and the Novikov ring used is $\mathbb{Z}_{2}\left[\pi_{2}(M)\right]$.

A stronger condition will play a key role in the following. Consider the quantum homology of $M$ with coefficients in $\Gamma=\mathbb{Z}_{2}\left[s^{-1}, s\right]$ with $\operatorname{deg}(s)=-2 C_{M}$ (where $C_{M}$ is the minimal Chern number). This is $Q H_{*}(M)=H_{*}\left(M ; \mathbb{Z}_{2}\right) \otimes \Gamma$.

Definition 1.1.5 With the notation above we say that $M$ is point invertible if [pt] is invertible in $Q H(M)$. This implies that there exists $0 \neq a_{0} \in H_{*}\left(M ; \mathbb{Z}_{2}\right), a_{1} \in$ $H_{*}\left(M ; \mathbb{Z}_{2}\right) \otimes \mathbb{Z}_{2}[s]$, and $k \in \mathbb{N}$ so that, if we put $a=a_{0}+a_{1} s$, then in $Q H_{*}(M)$ we have

$$
[\mathrm{pt}] * a=[M] s^{k / 2 C_{M}} .
$$

The natural number $k$ above is uniquely defined and we specify it by saying that $M$ is point invertible of order $k$.

Of course, as indicated above, a point invertible manifold is 2 -uniruled. The class of point invertible manifolds includes, for example, $\mathbb{C} P^{n}$ and the quadric $Q^{2 n} \subset \mathbb{C} P^{n+1}$. Moreover, in view of the product formula for Gromov-Witten invariants, this class is closed with respect to products. 
In general, no such direct algebraic criteria can be found to test the existence of mixed uniruling of the pair $(M, L)$ or even whether $L$ itself is uniruled because relative Gromov-Witten invariants are not well-defined in full generality.

\subsection{Main results}

Recall that by the work of Oh [42] if $L \subset M$ is a monotone Lagrangian, which we will assume from now on, then the Floer homology $H F(L):=H F(L, L)$ with $\mathbb{Z}_{2}$-coefficients is well-defined (the construction will be briefly recalled later in the paper). Floer homology is easily seen to be isomorphic (in general not canonically) to a quotient of a sub-vector space of $H\left(L ; \mathbb{Z}_{2}\right) \otimes \Lambda$. Here $H\left(L ; \mathbb{Z}_{2}\right)$ is singular homology and $\Lambda=\mathbb{Z}_{2}\left[t^{-1}, t\right]$ where the degree of $t$ is $|t|=-N_{L}$ (see Section $3.2(\mathrm{~g}$ ) for the precise definition). Thus, there are two extremal cases:

Definition 1.2.1 If $H F(L)=0$ we say that $L$ is narrow; if there exists an isomorphism $H F(L) \cong H\left(L ; \mathbb{Z}_{2}\right) \otimes \Lambda$, then we call $L$ wide. Note that the latter isomorphism is not required to be canonical in any sense.

Remarkably, all known monotone Lagrangians are either narrow or wide. We will see that the dichotomy narrow-wide plays a key role in structuring the properties of monotone Lagrangians. In particular, narrow Lagrangians tend to be small in the sense that their width is bounded and non-narrow ones tend to be barriers in the sense of Biran [7]: the width of their complement tends to be smaller than that of the ambient manifold. Wide Lagrangians are even more rigid.

1.2.1 Geometric rigidity We start with one result concerning narrow Lagrangians which also shows that the "narrow-wide" dichotomy holds in a variety of cases (related results are due to Buhovsky [15]):

Theorem 1.2.2 Let $L^{n} \subset M^{2 n}$ be a monotone Lagrangian. Assume that its singular homology $H_{*}\left(L ; \mathbb{Z}_{2}\right)$ is generated as a ring (with the intersection product) by $H_{\geq n-l}\left(L ; \mathbb{Z}_{2}\right)$.

(i) If $N_{L}>l$, then $L$ is either wide or narrow. Moreover, if $N_{L}>l+1$, then $L$ is wide.

(ii) In case $L$ is narrow, then $L$ is uniruled of order $K$ with $K=\max \{l+1$, $\left.n+1-N_{L}\right\}$ if $N_{L}<l+1$, and $K=l+1$ if $N_{L}=l+1$. Moreover, $w(L) \leq$ $2 K \eta$ where $\eta$ is the monotonicity constant. In particular, the width of narrow monotone Lagrangians $L$ is "universally" bounded: $w(L) \leq 2(n+1) \eta$. In case $L$ is narrow and not a homology sphere the bound can be improved to $w(L) \leq 2 n \eta$. 
Note that the finiteness of $w(L)$ from point (ii) is not trivial since $M$ is not assumed to be compact nor of finite volume or width. Moreover, when $L$ is not narrow, $w(L)$ might be infinite. For example, zero-sections in cotangent bundles (which are wide) have infinite width. A class of Lagrangians for which Theorem 1.2.2 gives nontrivial information is that of monotone Lagrangian tori. In this case $H_{*}\left(L ; \mathbb{Z}_{2}\right)$ is generated by $H_{\geq n-1}\left(L ; \mathbb{Z}_{2}\right)$ hence we can take $l=1$. As $N_{L} \geq 2>l$ we see that any monotone Lagrangian torus is either narrow or wide. In case such a Lagrangian is narrow we have $w(L) \leq 4 \eta$.

To obtain any meaningful uniruling results for Lagrangians which are not narrow, the same example of zero sections in cotangent bundles shows that some additional conditions need to be imposed on the ambient manifold $M$.

Theorem 1.2.3 Let $L$ be a monotone Lagrangian in a symplectic manifold $M$ which is point invertible of order $k$.

(i) If $L$ is not narrow, then $(M, L)$ is uniruled of type $(1,0)$ of order $<k$. In particular,

$$
w(M \backslash L) \leq\left(k-N_{L}\right) \eta .
$$

(ii) If $L$ is wide, then $L$ is uniruled of order $<k$ and we have

$$
w(L)+2 w(M \backslash L) \leq 2 k \eta .
$$

We emphasize that the somewhat surprising part of the statement is that the uniruling involving $L$ is of order strictly lower than $k$ whenever $M$ is point invertible of order precisely $k$ (in particular, it might happen that $M$ itself is uniruled of order precisely $k$ ).

Remark 1.2.4 (a) There are a few additional immediate inequalities that are worth mentioning: as $M$ is uniruled we have $w(M) \leq k \eta$ and so $w(L) \leq k \eta$. Moreover, as $M$ is 2 -uniruled, we have $w\left(M, \varnothing ;\left(r_{1}, r_{2} ; \varnothing\right)\right) \leq k \eta$. Obviously, we always have $w\left(M, \varnothing ;\left(r_{1}, r_{2} ; \varnothing\right)\right) \geq w\left(M, L ;\left(r_{1} ; r_{2}\right)\right)$.

(b) These general inequalities do not imply the inequality (2). Indeed, in contrast to $w\left(M, L ;\left(r_{1} ; r_{2}\right)\right)$, the two balls involved in estimating separately the width of $L$ and that of its complement are not required to be disjoint !

(c) A nontrivial consequence of point (i) of the Theorem is that if $M$ is point invertible of order $k$ and $L$ is non-narrow, then $N_{L} \leq k / 2$.

(d) Assuming the setting of the point (ii) of the Theorem we deduce from the fact that $L$ is uniruled of order $<k$, that $w(L) \leq 2\left(k-N_{L}\right) \eta$. However, this inequality lacks interest because $2\left(k-N_{L}\right) \geq k$ (since $\left.k \geq 2 N_{L}\right)$. 
1.2.2 Corollaries for Lagrangians in $\mathbb{C} P^{n}$ We endow $\mathbb{C} P^{n}$ with the standard Kähler symplectic structure $\omega_{\mathrm{FS}}$ normalized so that $\int_{\mathbb{C} P^{1}} \omega_{\mathrm{FS}}=1$. With this normalization we have $\mathbb{C} P^{n} \backslash \mathbb{C} P^{n-1} \approx \operatorname{Int} B^{2 n}(1 / \sqrt{\pi})$ hence $w\left(\mathbb{C} P^{n}\right)=1$. Note also that for every monotone Lagrangian $L \subset \mathbb{C} P^{n}$ we have $\eta=1 /(2 n+2)$ and that $\mathbb{C} P^{n}$ is point invertible of order $k=2 n+2$.

Corollary 1.2.5 Let $L$ be a monotone Lagrangian in $\mathbb{C} P^{n}$.

(i) At least one of the following inequalities is satisfied:

(a) $w(L) \leq n /(n+1)$.

(b) $\quad w\left(\mathbb{C} P^{n} \backslash L\right) \leq n /(n+1)$. Moreover, if $L$ is not narrow then possibility (b) holds and in fact we have

$$
w\left(\mathbb{C} P^{n} \backslash L\right) \leq \frac{\left[\frac{2 n}{N_{L}}\right] N_{L}}{2(n+1)} .
$$

(ii) If $L$ is wide, then we have

$$
w(L)+2 w\left(\mathbb{C} P^{n} \backslash L\right) \leq 2 .
$$

In case $L$ is not narrow, the inequality $w\left(\mathbb{C} P^{n} \backslash L\right) \leq n /(n+1)$ follows directly from Theorem 1.2.3. If $L$ is narrow, as $L$ cannot be a homology sphere (see eg Biran and Cieliebak [9]) we can take $l=n-1$ in Theorem 1.2.2 which then implies the inequality at (i) (a) above. Point (ii) of the Corollary follows from point (ii) of Theorem 1.2.3.

Corollary 1.2.5 implies in particular that for any monotone Lagrangian in $\mathbb{C} P^{n}$ we have

$$
w(L)+w\left(\mathbb{C} P^{n} \backslash L\right) \leq 1+\frac{n}{n+1}=2-\frac{1}{n+1}
$$

or, in other words, any monotone Lagrangian in $\mathbb{C} P^{n}$ is either a barrier (in the sense of [7]) or its width is strictly smaller than that of the ambient manifold. For example, $\mathbb{R} P^{n} \subset \mathbb{C} P^{n}$ satisfies $w\left(\mathbb{R} P^{n}\right)=1$ and $w\left(\mathbb{C} P^{n} \backslash \mathbb{R} P^{n}\right)=1 / 2$; for the Clifford torus

$$
\mathbb{T}_{\text {clif }}^{n}=\left\{\left[z_{0}: \cdots: z_{n}\right] \in \mathbb{C} P^{n}|| z_{0}|=\cdots| z_{n} \mid\right\}
$$

we have $w\left(\mathbb{T}_{\text {clif }}^{n}\right) \leq 2 /(n+1)$ (an explicit construction due to Buhovsky [16] shows that we actually have an equality here) and $w\left(\mathbb{C} P^{n} \backslash \mathbb{T}_{\text {clif }}^{n}\right)=n /(n+1)$ so that for $n=2$ both (a) and (b) are sharp. Both $\mathbb{R} P^{n}$ and $\mathbb{T}_{\text {clif }}^{n}$ show that the inequality at (ii) is sharp. We do not know if the inequality (3) is sharp. 
1.2.3 Spectral rigidity To summarize the results above, monotone non-narrow Lagrangians (at least) in appropriately uniruled symplectic manifolds are geometrically rigid. Of course, by standard Floer intersection theory, monotone Lagrangians which are not narrow, are also rigid in the sense that such a Lagrangian cannot be disjoined from itself by Hamiltonian deformation. We now present a different type of rigidity.

Let $\widetilde{\operatorname{Ham}}(M)$ be the universal cover of the Hamiltonian diffeomorphism group of a symplectic manifold $M$. Recall that, by work of Oh [47] and Schwarz [49] we can associate to any $\phi \in \widetilde{\operatorname{Ham}}(M)$ and any singular homology class $\alpha \in H_{*}\left(M ; \mathbb{Z}_{2}\right)$ a spectral invariant, $\sigma(\alpha, \phi) \in \mathbb{R}$. See Section 5.3 for the definition.

Here are two natural notions measuring the variation of an element $\phi \in \widetilde{\operatorname{Ham}}(M)$ on a Lagrangian submanifold $L \subset M$.

Definition 1.2.6 The depth and, respectively, the height of $\phi$ on $L$ are

$$
\begin{aligned}
\operatorname{depth}_{L}(\phi) & =\sup _{[H]=\phi} \inf _{\gamma \in \Gamma(L)} \int_{S^{1}} H(\gamma(t), t) d t \\
\text { height }_{L}(\phi) & =\inf _{[H]=\phi} \sup _{\gamma \in \Gamma(L)} \int_{S^{1}} H(\gamma(t), t) d t,
\end{aligned}
$$

where $\Gamma(L)$ stands for the space of smooth loops $\gamma: S^{1} \rightarrow L, H: M \times S^{1} \rightarrow \mathbb{R}$ is a normalized Hamiltonian, and the equality $[H]=\phi$ means that the path of Hamiltonian diffeomorphisms induced by $H, \phi_{t}^{H}$, is in the (fixed ends) homotopy class $\phi$.

Theorem 1.2.7 Let $L \subset M$ be a monotone non-narrow Lagrangian. Then for every $\phi \in \widetilde{\operatorname{Ham}}(M)$ :

(i) We have $\sigma([M], \phi) \geq \operatorname{depth}_{L}(\phi)$.

(ii) If $M$ is point invertible of order $k$, then

$$
\sigma([\mathrm{pt}], \phi) \geq \operatorname{depth}_{L}(\phi)-k \eta
$$

We will actually prove a more general statement than the one contained in Theorem 1.2.7, however, even this already has a nontrivial consequence.

Corollary 1.2.8 Any two non-narrow monotone Lagrangians in $\mathbb{C} P^{n}$ intersect.

Here is a quick proof of this Corollary. First, the theory of spectral invariants shows that for any manifold $M$ so that $Q H_{2 n}(M)=\mathbb{Z}_{2}[M]$ and any $\phi \in \widetilde{\operatorname{Ham}}(M)$ we have $\sigma\left([\mathrm{pt}], \phi^{-1}\right)=-\sigma([M], \phi)$. This is the case for $M=\mathbb{C} P^{n}$ and thus, as for $\mathbb{C} P^{n}$ we have $k=2 n+2, \eta=1 /(2 n+2)$, by Theorem 1.2.7 (ii) we deduce for any $\phi$ : 
$\sigma\left(\left[\mathbb{C} P^{n}\right], \phi\right)=-\sigma\left([\mathrm{pt}], \phi^{-1}\right) \leq-\operatorname{depth}_{L}\left(\phi^{-1}\right)+1=\operatorname{height}_{L}(\phi)+1$. Therefore, we have the inequalities

$$
\operatorname{depth}_{L}(\phi) \leq \sigma\left(\left[\mathbb{C} P^{n}\right], \phi\right) \leq \operatorname{height}_{L}(\phi)+1 .
$$

Assume now that $L_{0}$ and $L_{1}$ are two non-narrow Lagrangians in $\mathbb{C} P^{n}$ and $L_{0} \cap L_{1}=$ $\varnothing$. In this case, for any two constants $C_{0}, C_{1} \in \mathbb{R}$ we may find a normalized Hamiltonian $H$ which is constant equal to $C_{0}$ on $L_{0}$ and is constant and equal to $C_{1}$ on $L_{1}$. We pick $C_{1}>C_{0}+1$. Applying the first inequality in (4) to $L_{1}$ and the second to $L_{0}$ we get

$$
C_{1} \leq \operatorname{depth}_{L_{1}}(\phi) \leq \sigma\left(\left[\mathbb{C} P^{n}\right], \phi^{H}\right) \leq \operatorname{height}_{L_{0}}(\phi)+1 \leq C_{0}+1
$$

which leads to a contradiction.

A more general intersection result based on a somewhat different argument is stated later in the paper, in Section 2.4.

Remark 1.2.9 (a) We expect that, at least under possibly stronger assumptions, the $\mathbb{Z}_{2}$-Floer homology of the two Lagrangians involved (when defined) is not zero. We have a different, more algebraic approach [11] to the result in Corollary 1.2.8 which should be helpful in settling this issue. However, this approach goes beyond the scope of this paper and so it will not be further discussed here (see also Remark 2.4.2).

(b) The argument for the proof given above to Corollary 1.2.8 has been first used by Albers in [2] in order to detect Lagrangian intersections and by Entov and Polterovich [30]; Entov and Polterovich first noticed that this Corollary follows from an early version of our theorem in [12] combined with the results in [30]. Using the terminology of [30], Theorem 1.2.7 implies that a monotone non-narrow Lagrangian is heavy. This is because $[M]$ is an idempotent which satisfies $\sigma([M], \phi) \geq \operatorname{depth}_{L}(\phi)$ for all $\phi$. Assume now, additionally, that $M$ is point invertible of order $k$ and moreover that for any $\phi \in \widetilde{\operatorname{Ham}}(M), \sigma\left([\mathrm{pt}], \phi^{-1}\right)=-\sigma([M], \phi)$. In this case, we deduce $\sigma([M], \phi)=-\sigma\left([\mathrm{pt}], \phi^{-1}\right) \leq-\operatorname{depth}_{L}\left(\phi^{-1}\right)+k \eta=\operatorname{height}_{L}(\phi)+k \eta$ so that $L$ is even super-heavy.

1.2.4 Existence of narrow Lagrangians Clearly, a displaceable Lagrangian is narrow. For general symplectic manifolds this is the only criterion for the vanishing of Floer homology that we are aware of. Unfortunately, except in very particular cases, this is not very efficient as, for a given Lagrangian it is very hard to test the existence of disjoining Hamiltonian diffeomorphisms. Because of this, till now there are very few examples of monotone, narrow Lagrangians inside closed symplectic manifolds. One very simple example is a contractible circle embedded in a surface of genus $\geq 1$. However, even in $\mathbb{C} P^{n}$ it is nontrivial to detect such examples. Corollary 1.2.8 yields 
as a byproduct many examples of such narrow monotone Lagrangians: if one monotone Lagrangian which is not narrow is known, it suffices to produce another monotone Lagrangian which is disjoint from it.

Example 1.2.10 There are narrow monotone Lagrangians in $\mathbb{C} P^{n}, n \geq 2$.

Such Lagrangians are obtained using the Lagrangian circle bundle construction from Biran [8]. Namely, we take any monotone Lagrangian $L_{0} \subset Q^{2 n-2}$ in the quadric hypersurface (eg a Lagrangian sphere) and then push it up to the normal circle bundle of the complex quadric hypersurface $Q^{2 n-2} \subset \mathbb{C} P^{n}$ of appropriate radius such as to get a monotone Lagrangian $L \subset \mathbb{C} P^{n}$ which is an $S^{1}$-bundle over $L_{0}$. As we will see, this produces a Lagrangian that does not intersect $\mathbb{R} P^{n}$, which in turn is wide. A detailed construction of narrow Lagrangians in $\mathbb{C} P^{n}$ along these lines is given in Section 6.4.

1.2.5 Methods of proof and homological calculations All our results are based on exploiting the following machinery. It is well-known that counting pseudo-holomorphic disks with Lagrangian boundary conditions (and appropriate incidence conditions) does not lead, in general, to Gromov-Witten type invariants as these counts strongly depend on the choices of auxiliary data involved (almost complex structures, cycles etc). However, the moduli spaces of pseudo-holomorphic disks are sufficiently well structured so that these counts appropriately understood can be used to define a chain complex which we call the pearl complex (this construction was initially proposed by Oh [44] following an idea of Fukaya and is a particular case of the more recent cluster complex of Cornea and Lalonde [24] called there linear clusters). The resulting homology $Q H(L)$ is an invariant which we call the quantum homology of $L$. The key bridge between the properties of the ambient manifold and those of the Lagrangian is provided by the fact that $Q H(L)$ has the structure of an augmented two-sided algebra over the quantum homology of the ambient manifold, $Q H(M)$, and, with adequate coefficients, is endowed with duality. At the same time, again with appropriate coefficients, $Q H(L)$ is isomorphic to the Floer homology $\operatorname{HF}(L, L)$ of the Lagrangian $L$ with itself. Moreover, many of the additional algebraic structures also have natural correspondents in Floer theory. However, the models based on actual pseudo-holomorphic disks rather than on Floer trajectories are much more efficient from the point of view of applications: they provide a passage from geometry to algebra which is sufficiently explicit so that, together with sometimes delicate algebraic arguments, they lead to the structural theorems listed before. Actually, in this paper we will not make any essential use of the fact that the Lagrangian quantum homology can be identified with the Floer homology.

The deeper reason why the models based on pseudo-holomorphic disks are so efficient has to do with the fact that they carry an intrinsic "positivity" which is algebraically 
useful and is inherited from the positivity of area (and Maslov index, in our monotone case) of $J$-holomorphic curves. These methods also allow us to compute explicitly the various structures involved in several interesting cases. In particular, for the Clifford torus in $\mathbb{T}_{\text {clif }} \subset \mathbb{C} P^{n}$, for Lagrangians, $L \subset \mathbb{C} P^{n}$ with $2 H_{1}(L ; \mathbb{Z})=0$, and for simply connected Lagrangians in the quadric $Q$. The results of these calculations will be stated in three Theorems in Section 2.3 once the algebraic structures involved are introduced. However, these calculations imply a number of homological rigidity results as well as some uniruling consequences which can be stated without further preparation and so we review these just below.

The first such corollary deals with Lagrangian submanifolds $L \subset \mathbb{C} P^{n}$ for which every $a \in H_{1}(L ; \mathbb{Z})$ satisfies $2 a=0$ (in short: " $2 H_{1}(L ; \mathbb{Z})=0$ "). It extends some earlier results obtained by other methods in Seidel [51] and Biran [8]. Before stating the result let us recall the familiar example of $\mathbb{R} P^{n} \subset \mathbb{C} P^{n}, n \geq 2$, which satisfies $2 H_{1}\left(\mathbb{R} P^{n} ; \mathbb{Z}\right)=0$.

Corollary 1.2.11 Let $L \subset \mathbb{C} P^{n}$ be a Lagrangian submanifold with $2 H_{1}(L ; \mathbb{Z})=0$. Then $L$ is monotone with $N_{L}=n+1$ and the following holds:

(i) There exists a map $\phi: L \rightarrow \mathbb{R} P^{n}$ which induces an isomorphism of rings on $\mathbb{Z}_{2}-$ homology: $\phi_{*}: H_{*}\left(L ; \mathbb{Z}_{2}\right) \stackrel{\cong}{\rightrightarrows} H_{*}\left(\mathbb{R} P^{n} ; \mathbb{Z}_{2}\right)$, the ring structures being defined by the intersection product. In particular we have $H_{i}\left(L ; \mathbb{Z}_{2}\right)=\mathbb{Z}_{2}$ for every $0 \leq i \leq n$, and $H_{*}\left(L ; \mathbb{Z}_{2}\right)$ is generated as a ring by $H_{n-1}\left(L ; \mathbb{Z}_{2}\right)$.

(ii) $L$ is wide. Therefore, as $N_{L}=n+1$ and in view of point (i) just stated, we have $H F_{i}(L, L) \cong \mathbb{Z}_{2}$ for every $i \in \mathbb{Z}$.

(iii) Denote by $h=\left[\mathbb{C} P^{n-1}\right] \in H_{2 n-2}\left(\mathbb{C} P^{n} ; \mathbb{Z}_{2}\right)$ the generator. Then $h \cap_{L}[L]$ is the generator of $H_{n-2}\left(L ; \mathbb{Z}_{2}\right)$. Here $\cap_{L}$ stands for the intersection product between elements of $H_{*}\left(\mathbb{C} P^{n} ; \mathbb{Z}_{2}\right)$ and $H_{*}\left(L ; \mathbb{Z}_{2}\right)$.

(iv) Denote by inc ${ }_{*}: H_{i}\left(L ; \mathbb{Z}_{2}\right) \rightarrow H_{i}\left(\mathbb{C} P^{n} ; \mathbb{Z}_{2}\right)$ the homomorphism induced by the inclusion $L \subset \mathbb{C} P^{n}$. Then inc $*$ is an isomorphism for every $0 \leq i=$ even $\leq n$.

(v) $\left(\mathbb{C} P^{n}, L\right)$ is $(1,0)$-uniruled of order $n+1$.

(vi) $L$ is $2-$ uniruled of order $n+1$. Moreover, given two distinct points $x, y \in L$, for generic $J$ there is an even but nonvanishing number of disks of Maslov index $n+1$ each of whose boundary passes through $x$ and $y$.

(vii) For $n=2,\left(\mathbb{C} P^{2}, L\right)$ is $(1,2)$-uniruled of order 6 .

Other than $L=\mathbb{R} P^{n}$ we are not aware of any other Lagrangian $L \subset \mathbb{C} P^{n}$ satisfying $2 H_{1}(L ; \mathbb{Z})=0$. In view of Corollary 1.2 .11 it is tempting to conjecture that the only 
Lagrangians $L \subset \mathbb{C} P^{n}$ with $2 H_{1}(L ; \mathbb{Z})=0$ are homeomorphic (or diffeomorphic) to $\mathbb{R} P^{n}$, or more daringly symplectically isotopic to the standard embedding of $\mathbb{R} P^{n} \hookrightarrow$ $\mathbb{C} P^{n}$. Note however that in $\mathbb{C} P^{3}$ there exists a Lagrangian submanifold $L$, not diffeomorphic to $\mathbb{R} P^{3}$, with $H_{i}\left(L ; \mathbb{Z}_{2}\right)=\mathbb{Z}_{2}$ for every $i$. This Lagrangian is a quotient of $\mathbb{R} P^{3}$ by the dihedral group $D_{3}$. It has $H_{1}(L ; \mathbb{Z}) \cong \mathbb{Z}_{4}$. This example is due to Chiang [20].

Our second corollary is concerned with the Clifford torus,

$$
\mathbb{T}_{\text {clif }}^{n}=\left\{\left[z_{0}: \cdots: z_{n}\right] \in \mathbb{C} P^{n}|| z_{0}|=\cdots=| z_{n} \mid\right\} \subset \mathbb{C} P^{n} .
$$

This torus is monotone and has minimal Maslov number $N_{\mathbb{T}_{\text {clif }}^{n}}=2$. As before, we endow $\mathbb{C} P^{n}$ with the standard symplectic structure $\omega_{\mathrm{FS}}$ normalized so that $\int_{\mathbb{C} P^{1}} \omega_{\mathrm{FS}}=1$.

Corollary 1.2.12 The Clifford torus $\mathbb{T}_{\text {clif }}^{n} \subset \mathbb{C} P^{n}$ is wide, $\left(\mathbb{C} P^{n}, \mathbb{T}_{\text {clif }}^{n}\right)$ is $(1,0)$ uniruled of order $2 n$ and $\mathbb{T}_{\text {clif }}^{n}$ is uniruled of order 2 . For $n=2,\left(\mathbb{C} P^{2}, \mathbb{T}_{\text {clif }}^{2}\right)$ is $(1,1)$-uniruled of order 4 . In particular, $w\left(\mathbb{C} P^{2}, \mathbb{T}_{\text {clif }}^{2}:(r, \rho)\right) \leq 2 / 3$.

Finally, we also indicate a result concerning Lagrangians in the smooth complex quadric hypersurface $Q^{2 n} \subset \mathbb{C} P^{n+1}$ endowed with the symplectic structure induced from $\mathbb{C} P^{n}$. The next corollary is concerned with Lagrangians $L \subset Q^{2 n}$ with $H_{1}(L ; \mathbb{Z})=0$. We recall the familiar example of a Lagrangian sphere in $Q^{2 n}$ which can be realized for example as a real quadric.

Corollary 1.2.13 Let $L \subset Q^{2 n}, n \geq 2$, be a Lagrangian submanifold with $H_{1}(L ; \mathbb{Z})=$ 0 . Then $L$ is wide and $(Q, L)$ is $(1,1)$-uniruled of order $2 n$. In particular, $w(Q, L$ : $(r, \rho)) \leq 1$. If we assume in addition that $n=\operatorname{dim}_{\mathbb{C}} Q$ is even, then we also have:

(i) $H_{*}\left(L ; \mathbb{Z}_{2}\right) \cong H_{*}\left(S^{n} ; \mathbb{Z}_{2}\right)$.

(ii) $L$ is 3 -uniruled of order $2 n$ (an so $\left.w\left(Q, L:\left(\varnothing ; \rho_{1}, \rho_{2}, \rho_{3}\right)\right) \leq 1\right)$.

\subsection{Structure of the paper}

The main results of the paper are stated in the introduction and in Section 2. Namely, in the second section, after some algebraic preliminaries we review in Section 2.2 the structure of Lagrangian quantum homology. This structure is needed to state in Section 2.3 three theorems containing explicit computations. Each one of the three corollaries already described in Section 1.2.5 is a consequence of one of these theorems. Section 2 concludes - in Section 2.4 - with the statement of a Lagrangian intersection result which is a strengthening of Corollary 1.2.8. 
In Section 3 and Section 4 we develop the tools necessary to prove the results stated in the first two sections. More precisely, Section 3 contains the justification of the structure of Lagrangian quantum homology. While we indicate the basic steps necessary to establish this structure, certain technical details are omitted. These details are contained in our preprint [12] and we have decided not to include them here because they are quite tedious and long and relatively unsurprising for specialists. The fourth section contains a number of auxiliary results which provide additional tools which are necessary to prove the theorems of the paper.

The actual proofs of the results stated in Section 1 and Section 2 are contained in Sections 5 and 6 . Namely, the fifth section contains the proofs of the three main structural Theorems stated in the introduction as well as that of the Lagrangian intersection result stated in Section 2.4 and the sixth section contains the proofs of the three "computational" theorems stated in Section 2.3 and that of their corresponding three Corollaries from Section 1.2.5. The construction of the example mentioned in Section 1.2.4 is also included here as well as a few other related examples.

Finally, in Section 7 we discuss some open problems derived from our work.

Acknowledgments The first author would like to thank Kenji Fukaya, Hiroshi Ohta and Kaoru Ono for valuable discussions on the gluing procedure for holomorphic disks. He would also like to thank Martin Guest and Manabu Akaho for interesting discussions and great hospitality at the Tokyo Metropolitan University during the summer of 2006.

Special thanks from both of us to Leonid Polterovich for interesting comments and his interest in this project from its early stages as well as for having pointed out a number of imprecisions in earlier versions of the paper. We thank Laurent Lazzarini, Peter Albers, Misha Entov and Joseph Bernstein for useful discussions. We also thank the FIM at ETH Zurich and the CRM at the University of Montreal for providing a stimulating working atmosphere which allowed us to pursue our collaboration in the academic year 2007-8. We thank the referee for a careful reading of the paper and for comments which helped to improve the exposition.

While working on this project our two children, Zohar and Robert, were born and by the time we finally completed this paper they had already celebrated their first birthdays. We would like to dedicate this work to them and to their lovely mothers, Michal and Alina.

The first author was partially supported by the ISRAEL SCIENCE FOUNDATION (grant No. 1227/06*); the second author was supported by an NSERC Discovery grant and a FQRNT Group Research grant. 


\section{Lagrangian quantum structures}

In this section we introduce the algebraic structures and invariants essential for our applications. We will then indicate the main ideas in the proof of the related statements as well as a few technical aspects. Full details appear in [12].

\subsection{Algebraic preliminaries}

We fix here algebraic notation and conventions which will be used in the paper.

2.1.1 Graded modules and chain complexes Let $\mathcal{R}$ be a commutative graded ring, ie $\mathcal{R}$ is a commutative ring with unity, $\mathcal{R}$ splits as $\mathcal{R}=\bigoplus_{i \in \mathbb{Z}} \mathcal{R}_{i}$, for every $i, j \in \mathbb{Z}$ we have $\mathcal{R}_{i} \cdot \mathcal{R}_{j} \subset \mathcal{R}_{i+j}$ and $1 \in \mathcal{R}_{0}$. By a graded $\mathcal{R}$-module we mean an $\mathcal{R}$-module $M$ which is graded $M=\bigoplus_{i \in \mathbb{Z}} M_{i}$ with each component $M_{i}$ being an $\mathcal{R}_{0}$-module and moreover for every $i, j \in \mathbb{Z}$ we have $\mathcal{R}_{i} \cdot M_{j} \subset M_{i+j}$.

The chain complexes $(\mathcal{C}, d)$ we will deal with will often be of the following type. Their underlying space $\mathcal{C}=\bigoplus_{i \in \mathbb{Z}} \mathcal{C}_{i}$ will be a graded $\mathcal{R}$-module, and moreover the differential $d$, when viewed as a map of the total space $d: \mathcal{C} \rightarrow \mathcal{C}$, is $\mathcal{R}$-linear. Since it is not justified to call such complexes $\mathcal{C}$ "chain complexes over $\mathcal{R}$ " (as each $\mathcal{C}_{i}$ is not an $\mathcal{R}$-module) we have chosen to call them $\mathcal{R}$-complexes. Note that $(\mathcal{C}, d)$ is in particular also a chain complex of $\mathcal{R}_{0}$-modules in the usual sense. Note also that the homology $H(\mathcal{C}, d)$ is obviously a graded $\mathcal{R}$-module.

Most of our chain complexes $(\mathcal{C}, d)$ will be free $\mathcal{R}$-complexes. By this we mean that (the total space of) the $\mathcal{R}$-complex $\mathcal{C}$ is a finite rank free module over $\mathcal{R}$. In other words $\mathcal{C}=G \otimes \mathcal{R}$ where $G$ is a graded finite dimensional $\mathbb{Z}_{2}$-vector space and the grading on $\mathcal{C}$ is induced from the grading of $G$ and from the grading of $\mathcal{R}$. The differential $d$ on $\mathcal{C}$ of course does not need to have the form $d=d_{G} \otimes 1$. In fact we can split $d$, in a unique way, as a (finite) sum of operators $d=\sum_{l \in \mathbb{Z}} \delta_{l}$ where $\delta_{l}: G_{*} \rightarrow G_{*-1+l} \otimes \mathcal{R}_{-l}$. (Here $G_{*}$ is identified with $G_{*} \otimes 1 \subset G_{*} \otimes \mathcal{R}_{0}$ and the operators $\delta_{l}$ are extended to $\mathcal{C}$ by linearity over $\mathcal{R}$ ). In most of the complexes below the operators $\delta_{l}$ will actually be given as $\delta_{l}=\sum_{j} \partial_{l, j} \otimes r_{l, j}$ with $\partial_{l, j}: G_{*} \rightarrow G_{*-1+l}$ and $r_{l, j} \in \mathcal{R}_{-l}$.

Finally, we say that the differential $d$ of a free $\mathcal{R}$-complex $(\mathcal{C}, d)$ is positive if $\delta_{l}=0$ for every $l<0$. In that case we will call the operator $\delta_{0}$ the classical component of $d$.

2.1.2 Coefficient rings Denote by $H_{2}^{D}(M, L) \subset H_{2}(M, L ; \mathbb{Z})$ the image of the Hurewicz homomorphisms $\pi_{2}(M, L) \longrightarrow H_{2}(M, L)$. Let $H_{2}^{D}(M, L)^{+}$be the monoid of all the elements $u$ so that $\omega(u) \geq 0$. Put $\Lambda^{+}=\mathbb{Z}_{2}\left[H_{2}^{D}(M, L)^{+} / \sim\right]$ with $\sim$ 
the equivalence relation $u \sim v$ if and only if $\mu(u)=\mu(v)$ and similarly $\Lambda=$ $\mathbb{Z}_{2}\left[H_{2}^{D}(M, L) / \sim\right]$. We grade these rings so that the degree of $u$ equals $-\mu(u)$. In practice we will use the following natural identifications: $\Lambda^{+} \cong \mathbb{Z}_{2}[t], \Lambda \cong \mathbb{Z}_{2}\left[t^{-1}, t\right]$ induced by $H_{2}^{D}(M, L) \ni u \rightarrow t^{\mu(u) / N_{L}}$. The grading here is chosen so that $\operatorname{deg} t=-N_{L}$.

As mentioned in the introduction, the quantum homology of the ambient manifold is naturally a module over the ring $\Gamma=\mathbb{Z}_{2}\left[s^{-1}, s\right]$ where the degree of $s$ is $-2 C_{M}$. There is an obvious embedding of rings $\Gamma \hookrightarrow \Lambda$ which is defined by $s \rightarrow t^{\left(2 C_{M}\right) / N_{L}}$. The same embedding also identifies the ring $\Gamma^{+}=\mathbb{Z}_{2}[s]$ with its image in $\Lambda^{+}$. Using this embedding we regard $\Lambda$ (respectively $\Lambda^{+}$) as a module over $\Gamma$ (respectively, over $\Gamma^{+}$) and we define the following obvious extensions of the quantum homology:

$$
Q H(M ; \Lambda)=H_{*}\left(M ; \mathbb{Z}_{2}\right) \otimes \Lambda=Q H_{*}(M) \otimes_{\Gamma} \Lambda, Q H\left(M ; \Lambda^{+}\right)=H_{*}\left(M ; \mathbb{Z}_{2}\right) \otimes \Lambda^{+} \text {. }
$$

We endow $Q H(M ; \Lambda)$ and $Q H\left(M ; \Lambda^{+}\right)$with the quantum intersection product $*$ (see McDuff and Salamon [40] for the definition). Notice that we work here with quantum homology (not cohomology), hence the quantum product $*: Q H_{k}(M ; \Lambda) \otimes$ $Q H_{l}(M ; \Lambda) \rightarrow Q H_{k+l-2 n}(M ; \Lambda)$ has degree $-2 n$. The unit is $[M] \in Q H_{2 n}(M ; \Lambda)$, thus of degree $2 n$.

While we will essentially stick with $\Lambda, \Lambda^{+}$in this paper, for certain applications it can be useful to also use larger rings which distinguish explicitly the elements in $H_{2}^{D}(M, L)$. This is done as follows. Let $H_{2}^{S}(M, L) \subset H_{2}(M ; \mathbb{Z})$ be the image of the Hurewicz homomorphism $\pi_{2}(M) \rightarrow H_{2}(M ; \mathbb{Z})$, and let $H_{2}^{S}(M)^{+} \subset H_{2}^{S}(M)$ be the semigroup consisting of classes $A$ with $c_{1}(A)>0$. Similarly, denote by $H_{2}^{D}(M, L)^{+} \subset H_{2}^{D}(M, L)$ the semigroup of elements $A$ with $\mu(A)>0$. Let $\widetilde{\Gamma}^{+}=$ $\mathbb{Z}_{2}\left[H_{2}^{S}(M)^{+}\right] \cup\{1\}$ be the unitary ring obtained by adjoining a unit to the nonunitary group ring $\mathbb{Z}_{2}\left[H_{2}^{S}(M)^{+}\right]$. Similarly we put $\tilde{\Lambda}^{+}=\mathbb{Z}_{2}\left[H_{2}^{D}(M, L)^{+}\right] \cup\{1\}$. We write elements $Q \in \widetilde{\Gamma}^{+}$and $P \in \widetilde{\Lambda}^{+}$as "polynomials" in the formal variables $S$ and $T$ :

$$
Q(S)=a_{0}+\sum_{c_{1}(A)>0} a_{A} S^{A}, \quad P(T)=b_{0}+\sum_{\mu(B)>0} b_{B} T^{B}, \quad a_{0}, a_{A}, b_{0}, b_{B} \in \mathbb{Z}_{2} .
$$

We endow these rings with the following grading:

$$
\operatorname{deg} S^{A}=-2 c_{1}(A), \quad \operatorname{deg} T^{B}=-\mu(B) .
$$

Note that these rings are smaller than the rings $\hat{\Gamma}^{\geq 0}=\mathbb{Z}_{2}\left[\left\{A \mid c_{1}(A) \geq 0\right\}\right]$ and $\hat{\Lambda}^{\geq 0}=\mathbb{Z}_{2}[\{B \mid \mu(B) \geq 0\}]$. For example, $\hat{\Lambda}^{\geq 0}$ and $\hat{\Gamma}^{\geq 0}$ might have many nontrivial elements in degree 0 , whereas in $\widetilde{\Gamma}^{+}$and $\widetilde{\Lambda}^{+}$the only such element is 1 .

Let $Q H\left(M ; \widetilde{\Gamma}^{+}\right)=H\left(M ; \mathbb{Z}_{2}\right) \otimes \widetilde{\Gamma}^{+}$be the quantum homology of $M$ with coefficients in $\widetilde{\Gamma}^{+}$endowed with the quantum product, which we still denote by $*$ (note that now $*$ 
takes into account the actual classes of holomorphic spheres not only their Chern numbers). We have a natural map $H_{2}^{S}(M)^{+} \rightarrow H_{2}^{D}(M, L)^{+}$which induces on $\tilde{\Lambda}^{+}$ a structure of a $\widetilde{\Gamma}^{+}$-module. Put $Q H\left(M ; \widetilde{\Lambda}^{+}\right)=Q H\left(M ; \widetilde{\Gamma}^{+}\right) \otimes_{\tilde{\Gamma}^{+}} \widetilde{\Lambda}^{+}$and endow it with the quantum intersection product, still denoted $*$. Note that the quantum product is well defined with this choice of coefficients, since by monotonicity Chern numbers of pseudo-holomorphic spheres are nonnegative and the only possible pseudo-holomorphic sphere with Chern number 0 is constant. We grade this ring with the obvious grading coming from the two factors.

The most general rings of coefficients relevant for this paper are rings $\mathcal{R}$ that are graded commutative $\widetilde{\Lambda}^{+}$-algebras. We will usually endow a graded commutative ring $\mathcal{R}$ with the structure of $\widetilde{\Lambda}^{+}$-algebra by specifying a graded ring homomorphism $q: \widetilde{\Lambda}^{+} \rightarrow \mathcal{R}$. Here are a few examples of such rings $\mathcal{R}$ which are useful in applications.

(1) Take $\mathcal{R}=\Lambda=\mathbb{Z}_{2}\left[t^{-1}, t\right]$, and define $q$ by $q\left(T^{A}\right)=t^{\mu(A) / N_{L}}$.

(2) Take $\mathcal{R}=\Lambda^{+}=\mathbb{Z}_{2}[t]$, and define $q$ as in (1).

(3) Take $\mathcal{R}=\mathbb{Z}_{2}\left[H_{2}^{D}(M, L)\right]$ with the obvious $\tilde{\Lambda}^{+}$-algebra structure. We denote this ring by $\hat{\Lambda}$.

Given a graded commutative $\widetilde{\Lambda}^{+}$-algebra $\mathcal{R}$ we extend the coefficients of the quantum homology of the ambient manifold by $Q H(M ; \mathcal{R})=Q H\left(M ; \tilde{\Lambda}^{+}\right) \otimes \tilde{\Lambda}^{+} \mathcal{R}$.

2.1.3 A useful filtration There is a natural decreasing filtration of $\Lambda^{+}$and $\Lambda$ by the degrees of $t$, ie

$$
\mathcal{F}^{k} \Lambda=\left\{P \in \mathbb{Z}_{2}\left[t, t^{-1}\right] \mid P(t)=a_{k} t^{k}+a_{k+1} t^{k+1}+\cdots\right\} .
$$

We will call this filtration the degree filtration. In a similar way we can define the analogous filtrations on any graded $\tilde{\Lambda}^{+}$-algebra $\mathcal{R}$. This filtration induces an obvious filtration on any free $\mathcal{R}$-module.

\subsection{Structure of Lagrangian quantum homology}

Let $f: L \rightarrow \mathbb{R}$ be a Morse function on $L$ and let $\rho$ be a Riemannian metric on $L$ so that the pair $(f, \rho)$ is Morse-Smale. We grade the elements of $\operatorname{Crit}(f)$ by $|x|=\operatorname{ind}_{f}(x)$. Fix also a generic almost complex structure $J$ compatible with $\omega$. We recall that as we work in the monotone case (which, with the conventions of this paper includes $\left.N_{L} \geq 2\right)$, the Floer homology $H F_{*}(L ; \mathcal{R})=H F_{*}(L, L ; \mathcal{R})$ is well defined and invariant whenever $\mathcal{R}$ is a commutative $\mathbb{Z}_{2}\left[H_{2}^{D}(M, L)\right]$-algebra (see Section 3.2 (g) for a rapid review of the construction). 
Theorem $\mathbf{A}$ Let $\mathcal{R}$ be a graded commutative $\tilde{\Lambda}^{+}$-algebra (eg $\mathcal{R}=\Lambda, \Lambda^{+}$, or $\hat{\Lambda}$ ). For a generic choice of the triple $(f, \rho, J)$ there exists a finite rank, free $\mathcal{R}$-chain complex

$$
\mathcal{C}(L ; \mathcal{R} ; f, \rho, J)=\left(\mathbb{Z}_{2}\langle\operatorname{Crit}(f)\rangle \otimes \mathcal{R}, d^{\mathcal{R}}\right)
$$

with grading induced by Morse indices on the left factor and the grading of $\mathcal{R}$ on the right. The differential $d^{\mathcal{R}}$ of this complex is positive (see Section 2.1.1) and its classical component coincides with the Morse-homology differential $d^{\text {Morse }} \otimes 1$ (see Section 2.1.1). Moreover, this complex has the following properties:

(i) The homology of this chain complex is a graded $\mathcal{R}$-module and is independent of the choices of $(f, \rho, J)$, up to canonical comparison isomorphisms. It will be denoted by $Q H_{*}(L ; \mathcal{R})$. There exists a canonical (degree preserving) augmentation $\epsilon_{L}: Q H_{*}(L ; \mathcal{R}) \rightarrow \mathcal{R}$ which is an $\mathcal{R}$-module map. Moreover, for $\mathcal{R}=\Lambda$ the augmentation $\epsilon_{L}$ is nontrivial whenever $Q H(L ; \Lambda) \neq 0$.

(ii) The homology $Q H(L ; \mathcal{R})$ has the structure of a two-sided algebra with a unity over the quantum homology of $M, Q H(M ; \mathcal{R})$. More specifically, for every $i, j, k \in \mathbb{Z}$ there exist $\mathcal{R}$-bilinear maps

$$
\begin{aligned}
& Q H_{i}(L ; \mathcal{R}) \otimes Q H_{j}(L ; \mathcal{R}) \rightarrow Q H_{i+j-n}(L ; \mathcal{R}), \quad \alpha \otimes \beta \mapsto \alpha \circ \beta, \\
& Q H_{k}(M ; \mathcal{R}) \otimes Q H_{j}(L ; \mathcal{R}) \rightarrow Q H_{k+j-2 n}(L ; \mathcal{R}), \quad a \otimes \alpha \mapsto a \circledast \alpha,
\end{aligned}
$$

where $n=\operatorname{dim} L$. The first map endows $Q H(L ; \mathcal{R})$ with the structure of a ring with unity. This ring is in general not commutative. The second map endows $Q H(L ; \mathcal{R})$ with the structure of a module over the quantum homology ring $Q H(M ; \mathcal{R})$. Moreover, when viewing these two structures together, the ring $Q H(L ; \mathcal{R})$ becomes a two-sided algebra over the ring $Q H(M ; \mathcal{R})$. (The definition of a two-sided algebra is given below, after the statement of the theorem.) The unity of $Q H(L ; \mathcal{R})$ has degree $n=\operatorname{dim} L$ and will be denoted by $[L]$.

(iii) There exists a map

$$
i_{L}: Q H_{*}(L ; \mathcal{R}) \rightarrow Q H_{*}(M ; \mathcal{R})
$$

which is a $Q H_{*}(M ; \mathcal{R})$-module morphism and which is induced by a chain map which is a deformation of the singular inclusion (viewed as a map between Morse complexes). Moreover, this map is determined by the relation

$$
\left\langle\mathrm{PD}(h), i_{L}(x)\right\rangle=\epsilon_{L}(h \circledast x)
$$

for $x \in Q H(L ; \mathcal{R}), h \in H_{*}(M)$, with $\mathrm{PD}(-)$ Poincaré duality and $\langle-,-\rangle$ the $\mathcal{R}$-linear extension of the Kronecker pairing (ie $\left\langle\mathrm{PD}(h), \sum_{r} z_{r} T^{r}\right\rangle=$ $\left.\sum_{r}\left\langle\mathrm{PD}(h), z_{r}\right\rangle T^{r}\right)$. 
(iv) The differential $d^{\mathcal{R}}$ respects the degree filtration and all the structures above are compatible with the resulting spectral sequences.

(v) The differential $d^{\mathcal{R}}$ is in fact defined over $\tilde{\Lambda}^{+}$in the sense that the relation between $\mathcal{C}(L ; \mathcal{R} ; f, \rho, J)$ and $\mathcal{C}\left(L ; \Lambda^{+} ; f, \rho, J\right)$ is that $\mathcal{C}(L ; \mathcal{R} ; f, \rho, J) \cong$ $\mathcal{C}\left(L ; \Lambda^{+} ; f, \rho, J\right) \otimes \tilde{\Lambda}^{+} \mathcal{R}$ and $d^{\mathcal{R}} \cong d^{\tilde{\Lambda}^{+}} \otimes$ id. Moreover, any graded $\tilde{\Lambda}^{+}$ algebra homomorphism $\mathcal{R} \rightarrow \mathcal{R}^{\prime}$ (eg the inclusion $\Lambda^{+} \rightarrow \Lambda$ ) induces in homology a canonical morphism $Q H(L ; \mathcal{R}) \rightarrow Q H\left(L ; \mathcal{R}^{\prime}\right)$.

(vi) If $\mathcal{R}$ is a commutative $\mathbb{Z}_{2}\left[H_{2}^{D}(M, L)\right]$-algebra (eg $\mathcal{R}=\Lambda$ ), then there exists an isomorphism

$$
Q H_{*}(L ; \mathcal{R}) \rightarrow H F_{*}(L ; \mathcal{R})
$$

which is canonical up to a shift in grading.

The existence of the morphism $Q H(L ; \mathcal{R}) \rightarrow Q H\left(L ; \mathcal{R}^{\prime}\right)$ at point $(\mathrm{v})$ of the Theorem is not a purely algebraic statement about extension of coefficients. Rather, it means that the canonical extension of coefficients morphisms $H_{*}(\mathcal{C}(L ; \mathcal{R} ; f, \rho, J)) \rightarrow$ $H_{*}\left(\mathcal{C}\left(L ; \mathcal{R}^{\prime} ; f, \rho, J\right)\right)$ do not depend on $(f, \rho, J)$ in the sense that they are compatible with the canonical comparison isomorphisms relating the homologies associated to any two triples $\left(f_{0}, \rho_{0}, J_{0}\right)$ and $\left(f_{1}, \rho_{1}, J_{1}\right)$. In view of point (v) we will denote from now on the differential $d^{\mathcal{R}}$ by $d$ whenever the ring $\mathcal{R}$ is fixed and there is no risk of confusion.

By a two-sided algebra $A$ over a ring $R$ we mean that $A$ is a module over $R$, that $A$ is also a (possibly noncommutative) ring, and the two structures satisfy the following compatibility conditions:

$$
\forall r \in R \text { and } a, b \in A \text { we have } r(a b)=(r a) b=a(r b) .
$$

In other words, the first identity means that $A$, when considered as a left module over $R$, is an algebra over $R$, and the second one means that $A$ continues to be an algebra over $R$ when viewed as a right module over $R$, where the left and right module operations are the same one.

Before going on any further we would like to point out that, the existence of a module structure asserted by Theorem A has already some nontrivial consequences. For instance, the fact that $Q H_{*}(L ; \Lambda)$ is a module over $Q H_{*}(M ; \Lambda)$ implies that if $a \in Q H_{k}(M ; \Lambda)$ is an invertible element of degree $k$, then the map $a \circledast(-)$ gives rise to isomorphisms $Q H_{i}(L ; \Lambda) \rightarrow Q H_{i+k-2 n}(L ; \Lambda)$ for every $i \in \mathbb{Z}$, or in other words, $Q H_{*}(L ; \Lambda)$ is $(k-2 n)$-periodic. In view of point (vi) of the theorem the same periodicity holds for the Floer homology $H F_{*}(L)$ too. Note that there is yet another obvious periodicity for $\mathrm{QH}_{*}(L)$ that always holds (regardless of the module structure). Namely multiplying 
by $t \in \Lambda$ always gives isomorphisms $Q H_{*}(L ; \Lambda) \cong Q H_{*-N_{L}}(L ; \Lambda)$. This follows immediately from the fact that $Q H(L ; \Lambda)$ is a graded $\Lambda$-module and that $t \in \Lambda_{-N_{L}}$ is invertible. The above two periodicities, when applied together, provide a powerful tool in the computations of our invariants.

In most of the applications below we will take the ring of coefficients $\mathcal{R}$ to be either $\Lambda$ or $\Lambda^{+}$. Therefore we will sometimes drop the ring of coefficients from the notation and use the following abbreviations:

$$
\begin{aligned}
\mathcal{C}(L ; f, \rho, J) & =\mathcal{C}(L ; \Lambda ; f, \rho, J), & Q H(L) & =Q H(L ; \Lambda), \\
\mathcal{C}^{+}(L ; f, \rho, J) & =\mathcal{C}\left(L ; \Lambda^{+} ; f, \rho, J\right), & Q^{+} H(L) & =Q H\left(L ; \Lambda^{+}\right) .
\end{aligned}
$$

We will call the complex $\mathcal{C}(L ; f, \rho, J)$ (respectively $\left.\mathcal{C}^{+}(L ; f, \rho, J)\right)$ the (positive) pearl complex associated to $f, \rho, J$ and we will call the resulting homology the (positive) quantum homology of $L$. In the perspective of [24; 25] the complex $\mathcal{C}(L ; f, \rho, J)$ corresponds to the linear cluster complex.

Remark 2.2.1 (a) The complex $\mathcal{C}(L ; f, \rho, J)$ was first suggested by Oh [44] (see also Fukaya [32]) and, from a more recent perspective, it is a particular case of the cluster complex as described by Cornea and Lalonde [24]. The module structure over $Q^{+} H(M)$ discussed at point (ii) is probably known by experts - at least in the Floer homology setting - but has not been explicitly described yet in the literature. The product at (ii) is a variant of the Donaldson product defined via holomorphic triangles - it might not be widely known in this form. The map $i_{L}$ at point (iii) is the analogue of a map first studied by Albers in [2] in the absence of bubbling. The spectral sequence appearing at (iv) is a variant of the spectral sequence introduced by $\mathrm{Oh}$ [43]. The compatibility of this spectral sequence with the product at point (ii) has been first mentioned and used by Buhovsky [15] and independently by Fukaya, Oh, Ohta and Ono [33]. The comparison map at (vi) is an extension of the PiunikinSalamon-Schwarz construction [48], it extends also the partial map constructed by Albers in [1] and a more general such map was described independently in [24] in the "cluster" context. We also remark that this comparison map (with coefficients in $\Lambda$ ) identifies all the algebraic structures described above with the corresponding ones defined in terms of the Floer complex.

(b) The isomorphism $Q H(L) \cong H F(L)$ at point (vi) of Theorem A is an important structural property of the Lagrangian quantum homology. However, we would like to point out that this property of $Q H(L)$ is in fact not used in any of the applications presented in this paper. There is only one minor exception to this rule. Namely, our definition of wide and narrow Lagrangians $L$ goes via $H F(L)$. However we could have defined these notions directly using $Q H(L)$, and actually in the rest of the paper 
this will be the more relevant definition. The reason we have chosen to define wide and narrow using Floer homology is two-fold. Firstly, Floer homology is already well known in symplectic topology, and we wanted to base the notions of wide and narrow on a familiar concept. Secondly, it is easier to produce examples of narrow Lagrangians this way, simply by using the fact that if a Lagrangian $L$ is Hamiltonianly displaceable then $H F(L)=0$.

We insist on separating between $H F$ and $Q H$ because we do not view our Lagrangian quantum homology as a Lagrangian intersections invariant. Moreover, the results in this paper suggest that Lagrangian quantum homology has applications beyond Lagrangian intersections and thus we believe that this homology should be developed and studied in its own right.

\subsection{Some computations}

Here we present a few explicit computations of the various quantum structures mentioned in Theorem A performed on three examples: Lagrangians $L \subset \mathbb{C} P^{n}$ with $2 H_{1}(L ; \mathbb{Z})=0\left(\right.$ eg $\left.L=\mathbb{R} P^{n}\right)$, the Clifford torus $\mathbb{T}_{\text {clif }}^{2} \subset \mathbb{C} P^{2}$ and Lagrangians $L$ in the quadric with $H_{1}(L ; \mathbb{Z})=0$ (eg spheres). The proofs of the three results listed here are given in Section 6. More results in this direction can be found in [12].

We work here over the ring $\Lambda$. We start with Lagrangians $L \subset \mathbb{C} P^{n}$ that satisfy $2 H_{1}(L ; \mathbb{Z})=0$. Recall from Corollary 1.2.11 that $Q H_{i}(L) \cong H F_{i}(L) \cong \mathbb{Z}_{2}$ for every $i \in \mathbb{Z}$. Denote by $\alpha_{i} \in Q H_{i}(L)$ the generator. Denote by $h=\left[\mathbb{C} P^{n-1}\right] \in$ $H_{2 n-2}\left(\mathbb{C} P^{n} ; \mathbb{Z}_{2}\right)$ the class of a hyperplane. Recall also that in the quantum homology $Q H\left(\mathbb{C} P^{n}\right)$ we have

$$
h^{* j}= \begin{cases}h^{\cap j}, & 0 \leq j \leq n, \\ {\left[\mathbb{C} P^{n}\right] s,} & j=n+1 .\end{cases}
$$

As we will see (and is stated in Corollary 1.2.11) $N_{L}=n+1$, thus the embedding $\Gamma \hookrightarrow \Lambda$ is given by $s \rightarrow t^{2}$. It follows that in $Q H\left(\mathbb{C} P^{n} ; \Lambda\right)$ the last relation of (7) becomes $h^{*(n+1)}=\left[\mathbb{C} P^{n}\right] t^{2}$. Finally note that both $h$ and $[\mathrm{pt}]$ are invertible elements in $Q H\left(\mathbb{C} P^{n}\right)$.

Theorem 2.3.1 Let $L \subset \mathbb{C} P^{n}$ be a Lagrangian with $2 H_{1}\left(L ; \mathbb{Z}_{2}\right)=0$. Then:

(i) For every $i, j \in \mathbb{Z}, \alpha_{i} \circ \alpha_{j}=\alpha_{i+j-n}$.

(ii) For every $i \in \mathbb{Z}, h \circledast \alpha_{i}=\alpha_{i-2}$.

Furthermore, denote by $h_{j} \in H_{j}\left(\mathbb{C} P^{n} ; \mathbb{Z}_{2}\right)$ the generator (so that $h_{2 n-2}=h, h_{2 k}=$ $h^{\cap(n-k)}, \forall 0 \leq k \leq n, h_{\text {odd }}=0$ etc.) then: 
(iii) For $n=$ even we have:

$$
\begin{array}{ll}
i_{L}\left(\alpha_{2 k}\right)=h_{2 k}, & \forall 0 \leq 2 k \leq n, \\
i_{L}\left(\alpha_{2 k+1}\right)=h_{2 k+n+2} t, & \forall 1 \leq 2 k+1 \leq n-1 .
\end{array}
$$

(iv) For $n=$ odd we have:

$$
\begin{array}{ll}
i_{L}\left(\alpha_{2 k}\right)=h_{2 k}+h_{2 k+n+1} t, & \forall 0 \leq 2 k \leq n, \\
i_{L}\left(\alpha_{2 k+1}\right)=0, & \forall k \in \mathbb{Z} .
\end{array}
$$

The next result describes our computations for, mainly, the 2-dimensional Clifford torus $\mathbb{T}_{\text {clif }}^{2} \subset \mathbb{C} P^{2}$.

Theorem 2.3.2 The Clifford torus $\mathbb{T}_{\text {clif }}^{n}$ is wide for every $n \geq 1$. Let

$$
w \in H_{2}\left(\mathbb{T}_{\text {clif }}^{2} ; \mathbb{Z}_{2}\right) \hookrightarrow Q H_{2}\left(\mathbb{T}_{\text {clif }} ; \mathbb{Z}_{2}\right)
$$

be the fundamental class. There are generators $a, b \in H_{1}\left(\mathbb{T}_{\text {clif }}^{2}, \mathbb{Z}_{2}\right) \cong Q H_{1}\left(\mathbb{T}_{\text {clif }}^{2}\right)$, and $m \in Q H_{0}\left(\mathbb{T}_{\text {clif }}^{2}\right)$ which together with $w$ generate $Q H\left(\mathbb{T}_{\text {clif }}^{2}\right)$ as a $\Lambda$-module and satisfy the following relations:

(i) $a \circ b=m+w t, b \circ a=m, a \circ a=b \circ b=w t, m \circ m=m t+w t^{2}$.

(ii) $h \circledast a=a t, h \circledast b=b t, h \circledast w=w t, h \circledast m=m t$. Here $h=\left[\mathbb{C} P^{1}\right] \in$ $H_{2}\left(\mathbb{C} P^{2} ; \mathbb{Z}_{2}\right)$ is the class of a projective line.

(iii) $i_{L}(m)=[\mathrm{pt}]+h t+\left[\mathbb{C} P^{2}\right] t^{2}, \quad i_{L}(a)=i_{L}(b)=i_{L}(w)=0$.

We remark that, as the formulas in (i) indicate, the quantum product on $Q H(L)$ is in general noncommutative (even if we work over $\mathbb{Z}_{2}$ ).

Remark 2.3.3 (a) The fact that the Clifford torus is wide and point (i) of Theorem 2.3.2 have been obtained before by Cho in $[21 ; 22]$ by a different approach. From the perspective of [22] the Clifford torus is a special case of a torus which appears as a fibre of the moment map defined on a toric variety. See also Cho [23] for related results in this direction.

(b) Given that $\mathbb{T}_{\text {clif }}^{2}$ is wide we have $Q H_{*}\left(\mathbb{T}_{\text {clif }}^{2}\right) \cong H_{*}\left(\mathbb{T}_{\text {clif }}^{2} ; \mathbb{Z}_{2}\right) \otimes \Lambda$. Note however that such an isomorphisms cannot be made canonical in all degrees (see also Section 6.2). Nevertheless there is a canonical embedding $H_{2}\left(\mathbb{T}_{\text {clif }}^{2}\right) \hookrightarrow Q H_{2}\left(\mathbb{T}_{\text {clif }}^{2}\right)$ and the isomorphism $Q H_{1}\left(\mathbb{T}_{\text {clif }}^{2}\right) \cong H_{1}\left(\mathbb{T}_{\text {clif }}^{2} ; \mathbb{Z}_{2}\right.$ ) is canonical. (See our papers [12;13] for more on this.) 
We now turn to the third example: Lagrangians in the quadric. Let $L \subset Q^{2 n}$ be a Lagrangian submanifold of the quadric (where $\operatorname{dim}_{\mathbb{R}} Q=2 n$ ) that satisfies $H_{1}(L ; \mathbb{Z})=$ 0 . Such Lagrangians are monotone and the minimal Maslov number is $N_{L}=2 n$. Recall that by Corollary 1.2.13 $L$ is wide hence $Q H_{*}(L) \cong\left(H\left(L ; \mathbb{Z}_{2}\right) \otimes \Lambda\right)_{*}$. As $\operatorname{deg} t=-2 n$ we have $Q H_{0}(L) \cong H_{0}\left(L ; \mathbb{Z}_{2}\right)$ and $Q H_{n}(L) \cong H_{n}\left(L ; \mathbb{Z}_{2}\right)$. Denote by $\alpha_{0} \in Q H_{0}(L)$ and $\alpha_{n} \in Q H_{n}(L)$ the respective generators. Finally, denote by $[\mathrm{pt}] \in H_{0}\left(Q ; \mathbb{Z}_{2}\right)$ the class of a point.

Theorem 2.3.4 Let $L \subset Q$ be as above. Then:

(i) $[\mathrm{pt}] \circledast \alpha_{0}=-\alpha_{0} t,[\mathrm{pt}] \circledast \alpha_{n}=-\alpha_{n} t$.

(ii) $i_{L}\left(\alpha_{0}\right)=[\mathrm{pt}]-[Q] t$, where $[Q] \in H_{2 n}\left(Q ; \mathbb{Z}_{2}\right)$ is the fundamental class.

(iii) If $n$ is even then $\alpha_{0} \circ \alpha_{0}=\alpha_{n} t$.

Remark 2.3.5 The significance of the signs in the formulae above comes from the fact that we expect our machinery to hold with coefficients in $\mathbb{Z}$ and, if so, these are the signs that we obtain when taking into account orientations. As we shall see these signs play a significant role in some applications - see Corollary* 7.0.2.

\subsection{A criterion for Lagrangian intersections}

We describe here a criterion for Lagrangian intersections which is somewhat more general than Corollary 1.2.8 and which is stated in terms of the machinery described in Theorem A.

Let $L_{0}, L_{1} \subset M$ be two monotone Lagrangian submanifolds. Let $\Lambda_{0}=\mathbb{Z}_{2}\left[t_{0}^{-1}, t_{0}\right]$, $\Lambda_{1}=\mathbb{Z}_{2}\left[t_{1}^{-1}, t_{1}\right]$ be the associated rings, graded by $\operatorname{deg} t_{0}=-N_{L_{0}}$ and $\operatorname{deg} t_{1}=-N_{L_{1}}$. Recall from Section 2.1.2 that we also have the ring $\Gamma=\mathbb{Z}_{2}\left[s^{-1}, s\right], \operatorname{deg} s=-2 C_{M}$, and that $\Lambda_{0}, \Lambda_{1}$ are $\Gamma$-modules. Consider now the ring $\Lambda_{0,1}=\Lambda_{0} \otimes_{\Gamma} \Lambda_{1}$ with the grading induced from both factors (it is easy to see that this grading is well defined). Equivalently,

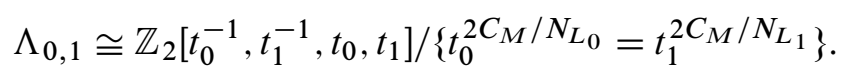

Note that $\Lambda_{0,1}$ is a $\Lambda_{0}$-algebra, a $\Lambda_{1}$-algebra as well as $\Gamma$-algebra. Thus we have well defined quantum homologies $Q H\left(L_{0} ; \Lambda_{0,1}\right), Q H\left(L_{1} ; \Lambda_{0,1}\right)$ as well as $Q H\left(M ; \Lambda_{0,1}\right)$.

With the above notation we have two canonical maps. The first one is the quantum inclusion $i_{L_{0}}: Q H_{*}\left(L_{0} ; \Lambda_{0,1}\right) \rightarrow Q H_{*}\left(M ; \Lambda_{0,1}\right)$, mentioned at point (iii) of Theorem A. The second map is $j_{L_{1}}: Q H_{*}\left(M ; \Lambda_{0,1}\right) \rightarrow Q H_{*-n}\left(L_{1} ; \Lambda_{0,1}\right)$, defined by $j_{L_{1}}(a)=a \circledast\left[L_{1}\right]$. Consider the composition

$$
j_{L_{1}} \circ i_{L_{0}}: Q H_{*}\left(L_{0} ; \Lambda_{0,1}\right) \longrightarrow Q H_{*-n}\left(L_{1} ; \Lambda_{0,1}\right) .
$$


Theorem 2.4.1 If $j_{L_{1}} \circ i_{L_{0}} \neq 0$, then $L_{0} \cap L_{1} \neq \varnothing$.

Remark 2.4.2 (a) We expect by [11] (also by [13]) that the condition $j_{L_{1}} \circ i_{L_{0}} \neq 0$ implies the nonvanishing of the Floer homology $\operatorname{HF}\left(L_{0}, L_{1}\right)$ (when defined and possibly under some additional restrictions).

(b) The map $j_{L_{1}}$ has appeared before in a different setting in the work of Albers [2].

Here is a consequence of this theorem which provides a different proof of Corollary 1.2.8. To state it we fix some more notation. As discussed before, for any Lagrangian submanifold the inclusion of the associated coefficient rings $\Lambda^{+} \rightarrow \Lambda$ induces a map of pearl complexes (when defined) $p: \mathcal{C}\left(L ; \Lambda^{+} ; f, \rho, J\right) \rightarrow \mathcal{C}(L ; \Lambda ; f, \rho, J)$ which is canonical in homology. Denote by $I Q^{+}(L)$ the image of $p_{*}: Q H\left(L ; \Lambda^{+}\right) \rightarrow$ $Q H(L ; \Lambda)$, the map induced in homology by $p$, and notice that $I Q^{+}(L)$ is a $\Lambda^{+}{ }_{-}$ module so that it makes sense to say whether a class $z \in I Q^{+}(L)$ is divisible by $t$ in $I Q^{+}(L)$ : this means that there is some $z^{\prime} \in I Q^{+}(L)$ so that $z=t z^{\prime}$.

Corollary 2.4.3 Let $L \subset M$ be a non-narrow monotone Lagrangian submanifold. Let $[\mathrm{pt}] \in Q H(M ; \Lambda)$ be the class of the point. If the product $[\mathrm{pt}] \circledast[L]$ is not divisible by $t^{2 C_{M} / N_{L}}$ in $I Q^{+}(L)$ then $L$ must intersect any non-narrow monotone Lagrangian in $M$.

Any non-narrow monotone Lagrangian $L \subset \mathbb{C} P^{n}$ satisfies the condition in the statement and so Corollary 2.4.3 implies Corollary 1.2.8. Indeed, put $z=[\mathrm{pt}] \circledast[L] \in I Q_{-n}^{+}(L)$. Assume that $z=t^{2 C_{\mathbb{C} P} / N_{L}} z^{\prime}$ for some $z^{\prime} \in I Q^{+}(L)$. We have $2 C_{\mathbb{C} P^{n}}=2 n+2$ and $\left|t^{2 C_{\mathbb{C} P}} / N_{L}\right|=-(2 n+2)$. Therefore, $\left|z^{\prime}\right|=-n+2 n+2=n+2$. But for degree reasons $I Q_{l}^{+}(L)=0$ for every $l>n$ and so $z^{\prime}=0$. In particular $z=0$. On the other hand as $[\mathrm{pt}] \in Q H(M ; \Lambda)$ is invertible and $[L] \neq 0$ we must have $z \neq 0$. A contradiction.

The proof of Corollary 2.4.3 is given in Section 5.4 after the proof of Theorem 2.4.1.

Remark 2.4.4 (a) By Theorem A, $L$ is non-narrow if and only if $[L] \neq 0 \in Q H(L)$. The reason is that $[L]$ is the unity of $Q H(L)$ when viewed as a ring. Moreover, whenever $M$ is point invertible and $L$ is not narrow the product $[\mathrm{pt}] \circledast[L]$ does not vanish. Of course, the nondivisibility condition in the statement of Corollary 2.4.3 is an additional strong restriction.

(b) The criterion in Corollary 2.4.3 does not apply to Lagrangians $L$ in the quadric which satisfy $H_{1}(L ; \mathbb{Z})=0$ so it does not lead to intersection results in this case. However, later in the paper (in Corollary* 7.0.2) we will see that Theorem 2.4.1 can also be applied to this setting but by working with integer coefficients, thus under the assumption that our machinery continues to work when taking into account orientations. 


\subsection{Simplification of notation}

As mentioned before, whenever we use the rings $\Lambda$ and $\Lambda^{+}$we will drop them from the notation in the following way:

$$
\begin{aligned}
\mathcal{C}(L ; f, \rho, J) & =\mathcal{C}(L ; \Lambda ; f, \rho, J), & Q H(L) & =Q H(L ; \Lambda), \\
\mathcal{C}^{+}(L ; f, \rho, J) & =\mathcal{C}\left(L ; \Lambda^{+} ; f, \rho, J\right), & Q^{+} H(L) & =Q H\left(L ; \Lambda^{+}\right) .
\end{aligned}
$$

Another simplification is the following. Theorem A involves three different algebraic operations: the quantum intersection product $*$, the Lagrangian quantum product $\circ$, and the external module operation $\circledast$ :

$$
\begin{aligned}
& *: Q H_{k}(M ; \mathcal{R}) \otimes Q H_{l}(M ; \mathcal{R}) \rightarrow Q H_{k+l-2 n}(M ; \mathcal{R}), \\
& \circ: Q H_{i}(L ; \mathcal{R}) \otimes Q H_{j}(L ; \mathcal{R}) \rightarrow Q H_{i+j-n}(L ; \mathcal{R}), \\
& \circledast: Q H_{k}(M ; \mathcal{R}) \otimes Q H_{j}(L ; \mathcal{R}) \rightarrow Q H_{i+j-2 n}(L ; \mathcal{R}) .
\end{aligned}
$$

As all these operations commute in the sense that $Q H(L ; \mathcal{R})$ is an algebra over $Q H(M ; \mathcal{R})$ we will sometimes denote all these operations by $*$.

\section{Sketch of proof for Theorem A}

We will explain the ideas behind the proof but, as mentioned in the introduction, we will not prove here this theorem in full. However, all the technical details which are omitted here can be found in [12]. The reason for proceeding in this way is that, on one hand, many of the actual technical verifications are not novel for specialists but quite long so including them here does not seem judicious. On the other hand, it is not possible to apply efficiently this theorem in the absence of a good understanding of the underlying moduli spaces and thus it is important to give a sufficiently detailed description of the construction of our machinery. We will also shortly review the main ideas behind the proof of transversality as well as the basic argument needed to prove the identities contained in the statement of the theorem.

\subsection{The moduli spaces}

It is useful to view our further constructions as a "quantum" version of standard constructions in Morse theory. In particular, in Morse theory, the Morse differential is modeled by a tree with one entry and one exit but no interior vertex. The same is true for a Morse morphism which relates two Morse complexes. The intersection product is modeled on trees with two entries and one exit. For the associativity of this product, are required trees with three entries and one exit. The quantum version of this construction 
consists in allowing each edge in these simple trees to be subdivided by a finite number of quantum contributions represented by pseudo-holomorphic disks or spheres. Such contributions can also appear at the vertices of the trees. Obviously, a more precise definition is required and we proceed to give one below.

A. Combinatorial preliminaries The trees needed here are of a reasonably simple type because we only use some rather elementary algebraic structures. The vertices of these trees will be of two types, corresponding to $J$-holomorphic disks (with boundary on $L$ ) or $J$-holomorphic spheres, and the edges will correspond to flow lines of Morse functions some defined on $L$ and some on $M$. The entries and the exit will correspond to critical points of these Morse functions. Here is a more precise description, unavoidably quite tedious. Conditions (i)-(iii) below simply model the data: each edge in the tree needs to carry a label (which geometrically corresponds to a particular Morse function). Each interior vertex will correspond to some $J$-holomorphic sphere or disk so that it needs to carry a label given by some homotopy class etc. A stability restriction is needed and is added as condition (iv). In the compactifications of such moduli spaces appear configurations where one (or more) edges are represented by flow lines of zero length. The corresponding geometric objects also appear by disk (or sphere) bubbling off. For our construction it is crucial that each configuration of this type appears exactly twice: once by bubbling off and once by the degeneration of a flow line. The purpose of condition (v) is to insure precisely this property. Point (vi) describes how the flow lines arriving at a vertex represented by a $J$-holomorphic curve are anchored to that curve.

Here are the precise details of the construction: We consider connected trees $\mathcal{T}$ with oriented edges embedded in $\mathbb{R} \times[0,1] \subset \mathbb{R}^{2}$ with entries lying on the line $\mathbb{R} \times\{1\}$ and a single exit which is situated on the line $\mathbb{R} \times\{0\}$ and so that the edges strictly decrease the $y$-coordinate. Clearly, at each internal vertex there is precisely one "exiting" (or departing) edge and at least one "entering" (or arriving) edge. There will be at most three entries and one exit. We call such a tree, $\mathcal{T},(M, L)$-labeled if the following additional structure is given:

- The entries and the exit have valence one (and they are the only vertices with this property). The vertices of the tree - except for the entries and the exit - are labeled by elements $\lambda \in H_{2}^{D}(M, L)$ or by elements $\mu \in H_{2}^{S}(M)$ with $\omega(\lambda) \geq 0$, $\omega(\mu) \geq 0$. The first kind of vertex will be called of disk type and the second will be called spherical. The set of vertices of $\mathcal{T}$ (including entries and the exit) is denoted by $v(\mathcal{T})$, the set of the spherical vertices is denoted by $v_{S}(\mathcal{T})$ and the set of disk type vertices is denoted by $v_{D}(\mathcal{T})$. The set of interior vertices will be denoted by $v_{\text {int }}(\mathcal{T})=v_{D}(\mathcal{T}) \cup v_{S}(\mathcal{T})$. The class of an interior vertex $v$ will be denoted by $[v] \in H_{2}^{D}(M, L)$ or $\in H_{2}^{S}(M)$. 
Let $\mathcal{F}_{L}$ be a finite set of Morse functions defined on $L$ and let $\mathcal{F}_{M}$ be a finite set of Morse functions defined on $M$. Put $\mathcal{F}=\mathcal{F}_{L} \cup \mathcal{F}_{M}$. An $(M, L)$-labeled tree $\mathcal{T}$ is called $\mathcal{F}$-colored if it satisfies the following three properties:

- The set of edges of $\mathcal{T}$ is denoted by $e(\mathcal{T})$ and is partitioned into two classes, the edges of type $L, e_{L}(\mathcal{T})$, and the edges of type $M, e_{M}(\mathcal{T})$. Each edge $e$ of type $L$ is colored by a Morse function $f_{e} \in \mathcal{F}_{L}$ and each edge $e$ of type $M$ is colored by a Morse function $f_{e} \in \mathcal{F}_{M}$. For $v \in v(\mathcal{T})$ we let $n_{L}(v)$ be the number of edges of type $L$ which are incident to $v$ and we let $n_{M}(v)$ be the number of those edges of type $M$. For an edge $e$ we let $e_{-} \in v(\mathcal{T})$ be the (initial) vertex where $e$ starts and we let $e_{+}$be the end (or final) vertex of $e$. If a vertex $v \in v_{S}(\mathcal{T})$, then $n_{L}(v)=0$. If $v \in v_{D}(\mathcal{T})$, then $n_{L}(v) \geq 1$. If $e \in e_{L}(\mathcal{T})$ and $e_{-}$(respectively $e_{+}$) is not an entry (respectively, not the exit), then $e_{-}$ (respectively $e_{+}$) belongs to $v_{D}(\mathcal{T})$.

- Each entry as well as the exit is labeled by a critical point of the Morse function corresponding to the incident edge. In other words, for all edges $e$, if $e_{-}$is an entry, then this implies that $e_{-}$is labeled by a critical point of the function $f_{e}$ and similarly for the exit. Any two distinct entries correspond to critical points of different Morse functions.

- At each vertex, distinct arriving edges are labeled by different Morse functions (but the exiting edge might be labeled with the same function as one of the arriving edges). If a vertex $v \in v_{D}(\mathcal{T})$ has the property $\omega([v])=0$ and $n_{L}(v) \leq 2$, then $n_{M}(v) \geq 1$. If a vertex $v \in v_{S}(\mathcal{T})$ has the property $\omega([v])=0$, then $n_{M}(v) \geq 3$.

The coloring of our trees will be usually described by means of an exit rule. Namely, fix as before a collection $\mathcal{F}$ of Morse functions (some on $L$, some on $M$ ). Notice that, for a planar tree $\mathcal{T}$, at each vertex $v$, the planarity of the tree induces an order among the arriving edges (by the values of the $x$-coordinates of the intersections of these edges with a horizontal line close to the vertex but above it).

- An exit rule $\Theta$ associates to each ordered vector, $\left(f_{1}, \ldots, f_{s}\right)$ with $f_{i} \in \mathcal{F}$, and symbol $S$ which can be either $L$ or $M$, a new function $\Theta\left(f_{1}, \ldots f_{s} ; S\right) \in \mathcal{F}$. An $\mathcal{F}$-colored tree $\mathcal{T}$ is called $\Theta$-admissible if, for each vertex of $\mathcal{T}$ whose exit edge is of type $S$ and whose arriving edges are colored, in order, by $\left(f_{1}, \ldots, f_{s}\right)$, the departing edge is colored by $\Theta\left(f_{1}, \ldots, f_{s} ; S\right) \in \mathcal{F}_{S}$.

Given an exit rule $\Theta$ notice that, for any $(M, L)$-labeled tree $\mathcal{T}$, if a coloring of the entry edges is given, then there exists a unique $\mathcal{F}$-coloring of $\mathcal{T}$ that is $\Theta$-admissible. Note also that, in order to color $\mathcal{T}$ in this way, we do not always need to know the 
value of $\Theta$ on all possible configurations (since some of them might not appear in any relevant trees).

We recall that the moduli spaces that we intend to construct consist of $J$-holomorphic disks and spheres joined by Morse trajectories. To proceed from trees to these moduli spaces we need an additional structure which describes how the flow lines are "anchored" to the $J$-curves. The structure in question is as follows:

- A marked point selector for an $\mathcal{F}$-colored tree $\mathcal{T}$ is given by an assignment $Q$ which associates to each vertex $v \in v_{S}(\mathcal{T})$ a collection $Q_{v}$ of distinct points in $S^{2}$ which is in 1-1 correspondence with the incident edges and, similarly, $Q$ associates to a vertex $v \in v_{D}(\mathcal{T})$ a collection $Q_{v} \subset D$ so that if an edge $e$ is of type $M$ its corresponding marked point is in $\operatorname{Int}(D)$ and if the edge $e$ is of type $L$ the corresponding marked point is in $\partial D$. Moreover, for $v \in v_{D}(\mathcal{T})$ the order among the marked points in $\partial D$ matches the order of the incident edges of type $L$ clockwise around the circle. If $e$ is an arriving edge (at some internal vertex) the respective marked point is denoted by $q_{+}(e)$ and if the edge is the exiting one, then the marked point is denoted by $q_{-}(e)$.

We denote $\mathcal{F}$-colored trees together with a marked point selector $Q$ by $(\mathcal{T}, Q)$ and we refer to the pair $(\mathcal{T}, Q)$ as an $\mathcal{F}$-colored tree with marked points. The marked point selectors that will be used here satisfy an additional property: they only depend on the type of the edge $e$, the valence of the vertex $v$, on whether the edge $e$ is an exit edge or an entry one and, in this last case, on the planar order of the edge among the arriving edges at the vertex $v$. In other words, we can view such a marked point selector as an abstract rule which associates a certain marked point to each edge incident to a vertex of any $\mathcal{F}$-colored tree. In view of this, if $Q$ and $Q^{\prime}$ are marked point selectors we can write $Q=Q^{\prime}$ if the two corresponding rules agree.

For a tree $\mathcal{T}$ we indicate its entries and the exit by a symbol like $(x, y, z: w)$ where the first components - in this case, they are three - are the labels of the entries written in the planar order and the last component indicates the label of the exit. We call this data the symbol of the tree $\mathcal{T}$. We denote the symbol of the $\mathcal{F}$-colored tree $\mathcal{T}$ by $\operatorname{symb}(\mathcal{T})$. The class of the tree $\mathcal{T},[\mathcal{T}] \in H_{2}^{D}(M, L)$ is defined to be the sum of the classes of the interior vertices.

B. Construction of the moduli spaces Fix an $\mathcal{F}$-colored tree with marked points $(\mathcal{T}, Q)$. Fix also a pair $\rho=\left(\rho_{M}, \rho_{L}\right)$ where $\rho_{L}$ is a Riemannian metric on $L$ and $\rho_{M}$ is a Riemannian metric on $M$. For every $f \in \mathcal{F}$ let $\gamma_{t}^{f}$ be the associated negative gradient flows (with respect to the metric $\rho_{L}$ for the functions defined on $L$ and with respect to the metric $\rho_{M}$ for the functions defined on $\left.M\right)$. Denote by $\left(x_{1}, \ldots, x_{l}: y\right)$ the symbol of $\mathcal{T}$. 
For an $\omega$-compatible almost complex structure $J$ and a class $\lambda \in H_{2}^{D}(M, L)$ (or in $H_{2}^{S}(M)$ ) let $\mathcal{M}(\lambda, J)$ be the moduli space of parametrized $J$-disks (respectively $J$-spheres) in the class $\lambda$.

The pearl moduli space modeled on $(\mathcal{T}, Q)$ will be denoted by $\mathcal{P}_{\mathcal{T}, Q}(J, \rho)$ (or, if the data involved is clear from the context, just $\mathcal{P}_{\mathcal{T}}$ ) and it is defined as follows. If $\mathcal{T}$ has no interior vertex or, equivalently, it consists of precisely one edge $e$ connecting the entry (which is labeled by a critical point $x=x_{1}$ of $\left.f_{e}\right)$ to the exit labeled by $y \in \operatorname{Crit}\left(f_{e}\right)$, then $\mathcal{P}_{\mathcal{T}}$ is the unparametrized moduli space of flow lines of $\gamma^{f_{e}}$ connecting $x$ to $y$. In case $\mathcal{T}$ contains an internal vertex, consider the product

$$
\Pi(\mathcal{T})=\prod_{v \in v_{\text {int }}(\mathcal{T})} \mathcal{M}([v], J)
$$

and let $S_{\mathcal{T}, Q}$ consist of all $\left\{u_{v}\right\}_{v \in v_{\text {int }}(\mathcal{T})} \in \Pi(\mathcal{T})$ subject to the constraints:

- For each internal edge $e \in e(\mathcal{T})$ there is $t \geq 0$ (called the length of $e$ ) such that

$$
\gamma_{t}^{f_{e}}\left(u_{e_{-}}\left(q_{-}(e)\right)\right)=u_{e_{+}}\left(q_{+}(e)\right) .
$$

- For an entry edge, $e$, let $x_{i}$ be the critical point labeling the vertex $e_{-}$. We have

$$
\lim _{t \rightarrow-\infty} \gamma_{t}^{f_{e}}\left(u_{e_{+}}\left(q_{+}(e)\right)\right)=x_{i}
$$

- For the exit edge $e$ we have

$$
\lim _{t \rightarrow \infty} \gamma_{t}^{f_{e}}\left(u_{e_{-}}\left(q_{-}(e)\right)\right)=y .
$$

Finally, define $\mathcal{P}_{\mathcal{T}, Q}=S_{\mathcal{T}, Q} / \sim$ where $\sim$ is given by the action of the obvious reparametrization groups which act on the $\mathcal{M}([v], J)$ 's and preserve the marked points. See Figure 1 for an example.

The moduli space $\mathcal{P}_{\mathcal{T}, Q}$ has a virtual dimension which only depends on the structure encoded in the definition of the colored trees with marked points. This virtual dimension will be denoted by $\delta(\mathcal{T})$. When transversality is achieved, it coincides with the actual manifold dimension of $\mathcal{P}_{\mathcal{T}, Q}$. As we will see in the next section, under this transversality assumption, the space $\mathcal{P}_{\mathcal{T}, Q}$ is a manifold, in general noncompact, with a boundary consisting of configurations where some edge of $\mathcal{T}$ has 0 -length.

Assume that the symbol of $\mathcal{T}$ is $\left(x_{1}, \ldots, x_{k}: y\right)$ and that there are $s$ entries among the $x_{i}$ 's which are critical points of functions in $\mathcal{F}_{M}$. Then the formula giving this virtual dimension is

$$
\delta(\mathcal{T})=\sum_{i}\left|x_{i}\right|-|y|+\mu[\mathcal{T}]+\epsilon(k)-(s+k-1) n
$$



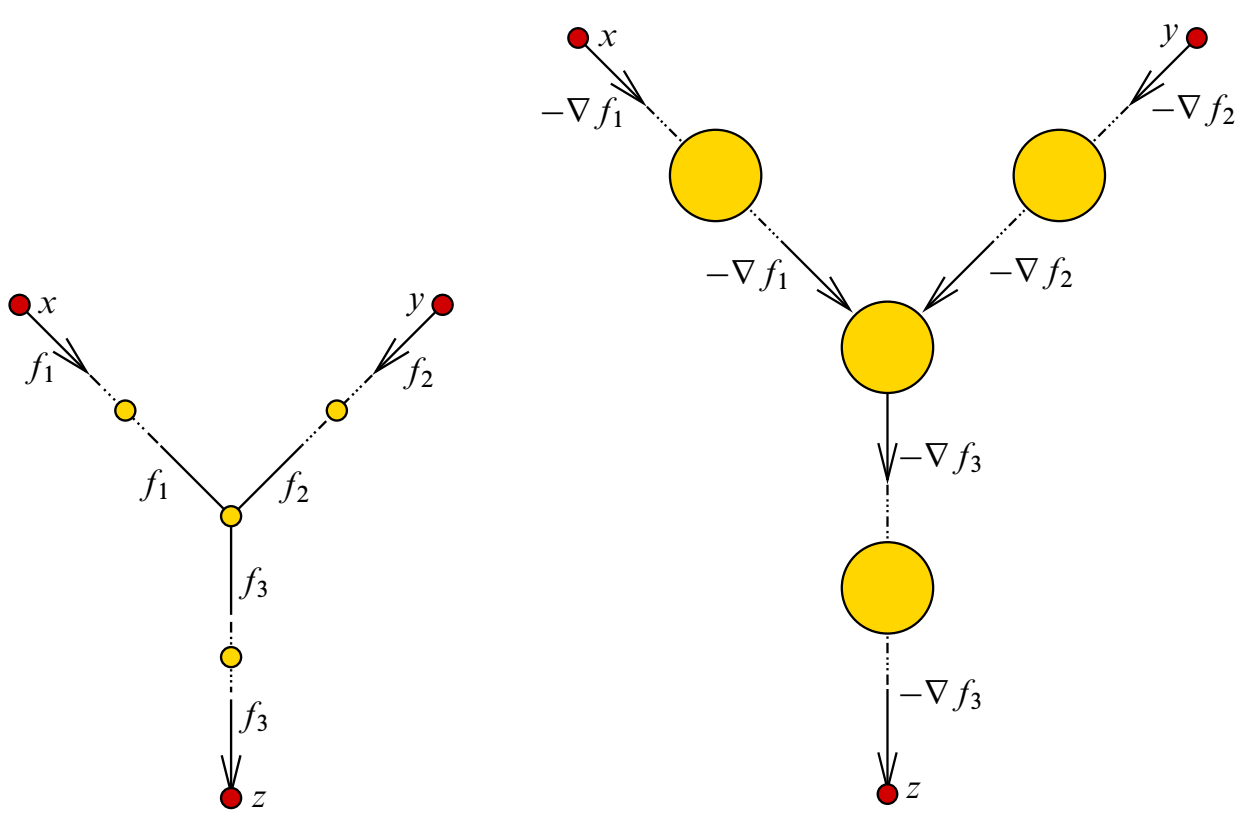

Figure 1: A tree of symbol $(x, y: z)$ on the left and a pearly trajectory corresponding to it on the right. See Section 3.2 (b) below for the choice of the labeling of the edges.

where $\epsilon(k)=-1$ if $k=1, y \in L$, and $\epsilon(k)=0$ otherwise.

C. Equivalence of trees In the sequel two $\mathcal{F}$-colored trees will be viewed as equivalent if the underlying topological trees are isomorphic by a tree isomorphism which preserves the order of the entering edges at each vertex and which also preserves the labels and the coloring.

Remark 3.1.1 Most of our moduli spaces are constructed according to the recipe above. In particular, they are all modeled on $(M, L)$-labeled trees. However, sometimes we need to work with variants of the last part of the construction. For example, we might use instead of Morse functions, Morse cobordisms; instead of a single almost complex structure we might require a family of such structures. Moreover, sometimes, some of the curves used in the construction satisfy a perturbed Cauchy-Riemann equation or the domains of some of the "vertices" in our trees will not be spheres or disks but rather, cylinders or strips etc. In all these cases we will describe explicitly the (generally minor) modifications that are needed in the construction above. 


\subsection{Definition of the algebraic structures}

The formalism given above allows us to define all the particular moduli spaces needed for our various operations and we will describe all these constructions below. In all these cases, we indicate the relevant moduli spaces by following the scheme above. In each case we will describe the various structures involved, namely, the class of Morse functions $\mathcal{F}$, the exit rule $\Theta$ (we will give its values only over that part of its domain which is relevant), the marked point selector $Q$ as well as the $\operatorname{symbol} \operatorname{symb}(\mathcal{T})$ of the relevant trees. We will also indicate in each case the formula for the virtual dimension of the respective moduli spaces.

The definitions of our operations and their properties depend on the transversality results which will be reviewed in the next section. Moreover, the various relations that need to be proved require to understand the compactification of these moduli spaces, a description of their boundary and a gluing formula. This part will be discussed in the last subsection.

We write the formulas below over $\tilde{\Lambda}^{+}-$see Section 2.1.2. Given any $\tilde{\Lambda}^{+}$-algebra $\mathcal{R}$ given by a graded ring homomorphism $q: \tilde{\Lambda}^{+} \rightarrow \mathcal{R}$, these formulas induce corresponding ones over $\mathcal{R}$ by simply replacing the formal variables $T^{A}, A \in H_{2}^{D}(M, L)$, by their values $q\left(T^{A}\right)$. As before, we fix a pair $\rho=\left(\rho_{L}, \rho_{M}\right)$ of Riemannian metrics on $L$ and on $M$ as well as an almost complex structure $J$ compatible with $\omega$.

(a) (The pearl complex and its differential) Here and in the points (b) and (c) below all the internal vertices are of disk type and all internal edges are of type $L$ so that we omit from the notation of $\Theta$ the symbol $S$ as $S=L$ in these three cases.

Consider a single Morse function $f: L \rightarrow \mathbb{R}$ and put $\mathcal{F}=\{f\}$. The pearl complex is

$$
\mathcal{C}\left(L ; \mathcal{R} ; f, \rho_{L}, J\right)=\left(\mathbb{Z}_{2}\langle\operatorname{Crit}(f)\rangle \otimes \mathcal{R}, d\right) .
$$

The differential $d$ is defined for generic choices of our data. To describe it, we consider $\mathcal{F}$-colored trees with marked points, $(\mathcal{T}, Q)$, with symbol $(x: y)$ with $x, y \in \operatorname{Crit}(f)$ and so that the marked point selector associates to each $e \in e(\mathcal{T}), q_{-}(e)=+1 \in \partial D$ and $q_{+}(e)=-1 \in \partial D$. See Figure 2 . It is easy to see that the virtual dimension of the associated moduli spaces is given by $\delta(\mathcal{T})=|x|-|y|+\mu[\mathcal{T}]-1$.

We now put

$$
d x=\sum_{y,(\mathcal{T}, Q)} \#_{2}\left(\mathcal{P}_{\mathcal{T}, Q}\right) y T^{[\mathcal{T}]}
$$

where, $y,(\mathcal{T}, Q)$ go over all the trees $(\mathcal{T}, Q)$ as above and we only count elements in $\mathcal{P}_{\mathcal{T}, Q}$ when the associated virtual dimension is 0 (we will use the same convention in 


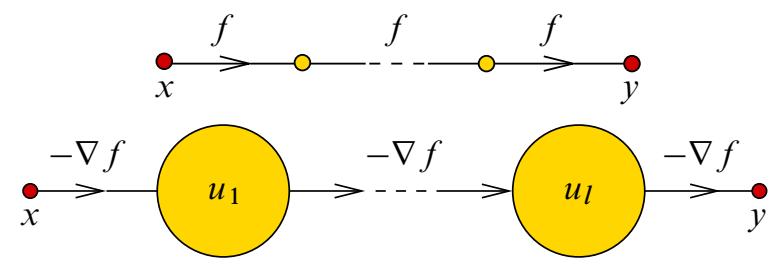

Figure 2: A tree of symbol $(x: y)$ at the top and a pearly trajectory corresponding to it at the bottom

the other examples below). The relation $d^{2}=0$ is based on the properties of the same type of moduli spaces but with virtual dimension equal to 1 . The necessary ingredients for this verification and the outline of the proof will be indicated in Section 3.3 and Section 3.4. Notice that if $f$ has a single maximum, $P$, then, for degree reasons, $P$ is a cycle in the pearl complex $\mathcal{C}\left(L ; \mathcal{R} ; f, \rho_{L}, J\right)$ (the point here is that the differential is defined over $\widetilde{\Lambda}^{+}$).

We will omit $L, J, \rho, \mathcal{R}$ from the notation if they are clear from the context.

(b) (The quantum product) In this case $\mathcal{F}=\left\{f_{1}, f_{2}, f_{3}\right\}$ with the three functions $f_{i}$ all defined on $L$. The product is defined by:

$$
\circ: \mathcal{C}\left(f_{1}\right) \otimes_{\mathcal{R}} \mathcal{C}\left(f_{2}\right) \rightarrow \mathcal{C}\left(f_{3}\right), \quad x \circ y=\sum_{z,(\mathcal{T}, Q)}\left(\#_{2} \mathcal{P}_{\mathcal{T}, Q}\right) z T^{[\mathcal{T}]}
$$

where the sum is taken over all the $\mathcal{F}$-colored trees with marked points $(\mathcal{T}, Q)$ of symbol $(x, y: z)$ with $x \in \operatorname{Crit}\left(f_{1}\right), y \in \operatorname{Crit}\left(f_{2}\right)$ and $z \in \operatorname{Crit}\left(f_{3}\right)$ which are $\Theta$-admissible with $Q$ and $\Theta$ as follows. First, the marking selector satisfies: if $e_{+}$is of valence at most 2 then $q_{+}(e)=-1 \in \partial D$; if $e_{-}$is of valence at most 3 , $q_{-}(e)=+1 \in \partial D$; if $e_{+}$is of valence 3 , and $e$ is the $j$-th entering edge (in the planar order) at the vertex $e_{+}$(clearly, $j \in\{1,2\}$ ), then $q_{+}(e)=e^{-2 \pi j / 3 i} \in \partial D$. In other words, at a vertex of valence 3 , the marked (or incidence) points are the roots of order three of the unity. Finally, the exit rule is $\Theta\left(f_{i}\right)=f_{i} \forall i \in\{1,2,3\}, \Theta\left(f_{1}, f_{2}\right)=f_{3}$. The virtual dimension in this case is $\delta(\mathcal{T})=|x|+|y|-|z|-n+\mu[\mathcal{T}]$. Schematically, the trees used here and the associated configurations are depicted in Figure 1.

Similar moduli spaces but of virtual dimension 1 are used to show that the linear map defined by (12) defines a chain morphism and thus descends to homology.

A useful remark here is that we can also use instead of the three functions $f_{1}, f_{2}, f_{3}$ only two function $f_{1}$ and $f_{2}$ with the same exit rule as above except that for the vertex of valence 3 we require $\Theta\left(f_{1}, f_{2}\right)=f_{2}$. It is easy to see that this definition provides 
a product

$$
\circ: \mathcal{C}\left(f_{1}\right) \otimes_{\mathcal{R}} \mathcal{C}\left(f_{2}\right) \rightarrow \mathcal{C}\left(f_{2}\right)
$$

which coincides in homology with the product given before (see also the invariance properties described at point (e)). This is particularly useful in verifying the associativity of the product as described at point (f) below as it allows one to work in that verification with only three Morse functions. Another reason why this description of the product is useful is that, assuming that $f_{1}$ has a single maximum $P$, we see that if a moduli space $\mathcal{P}_{\mathcal{T}, Q}$ used to define (13) is of symbol $(P, y: z)$ and of dimension 0 , then $y=z$ and $\mathcal{P}_{\mathcal{T}, Q}$ consists of the unique Morse trajectory of $f_{1}$ joining $P$ to $y$. Thus $P \circ y=y$ hence $P$ is a unity at the chain level for the product defined in (13).

(c) (The module structure) We now have $\mathcal{F}=\left\{f_{1}, f_{2}\right\}$ with one Morse function $f_{1}: M \rightarrow \mathbb{R}$ and one Morse function $f_{2}: L \rightarrow \mathbb{R}$. Let $C M\left(f_{1} ; \mathcal{R}\right)=\mathbb{Z}_{2}\left\langle\operatorname{Crit}\left(f_{1}\right)\right\rangle \otimes \mathcal{R}$ be the Morse complex of $f_{1}$ tensored with the ring $\mathcal{R}$ (endowed with the Morse differential $d=d_{\text {Morse }} \otimes 1$ ). The module action is defined by

$$
\circledast: C M\left(f_{1}\right) \otimes_{\mathcal{R}} \mathcal{C}\left(f_{2}\right) \rightarrow \mathcal{C}\left(f_{2}\right), \quad a \circledast x=\sum_{y,(\mathcal{T}, Q)}\left(\#_{2} \mathcal{P}_{\mathcal{T}}\right) \text { y } T^{[\mathcal{T}]}
$$

where the sum is taken over all the $\mathcal{F}$-colored trees $(\mathcal{T}, Q)$ of symbol $(a, x: y)$ with $a \in \operatorname{Crit}\left(f_{1}\right)$ and $x, y \in \operatorname{Crit}\left(f_{2}\right)$ which are $\Theta$-admissible for $Q$ and $\Theta$ defined as follows: for all edges $e$ of type $L, q_{+}(e)=-1 \in \partial D, q_{-}(e)=+1 \in \partial D$; if $e$ is an edge of type $M$ (there can in fact be at most one such edge), then $q_{+}(e)=0 \in D$; $\Theta\left(f_{2}\right)=f_{2}, \Theta\left(f_{1}, f_{2}\right)=f_{2}$. See Figure 3. The virtual dimension in this case is $\delta=|a|+|x|-|y|-2 n+\mu([\mathcal{T}])$.

The same type of moduli spaces but of virtual dimension 1 serve to prove that this operation passes to homology. However, at this step a modification is needed and has to do with the proof of transversality: we need that in these moduli spaces if a vertex $v$ is of valence three, then the corresponding curve $u_{v}$ is not pseudo-holomorphic but rather it carries a small Hamiltonian perturbation of type

$$
\left\{\begin{array}{l}
u:(D, \partial D) \rightarrow(M, L), \\
\partial_{s} u+J(u) \partial_{t} u=-X_{F}(s, t, u)-J(u) X_{G}(s, t, u)
\end{array}\right.
$$

with $F, G: D \times M \rightarrow \mathbb{R}$ well chosen Hamiltonians and $X_{F}$ and $X_{G}$ the respective Hamiltonian vector fields (see McDuff and Salamon [40] and Biran and Cornea [12] for details). The reason why these perturbations are needed will be explained in the next section and we refer to [12] for the full construction.

(d) (The inclusion $i_{L}$ ) In this case we use one Morse function $f_{1}: L \rightarrow \mathbb{R}$ and another Morse function $f_{2}: M \rightarrow \mathbb{R}$ and $\mathcal{F}=\left\{f_{1}, f_{2}\right\}$. The relevant $\mathcal{F}$-colored trees 


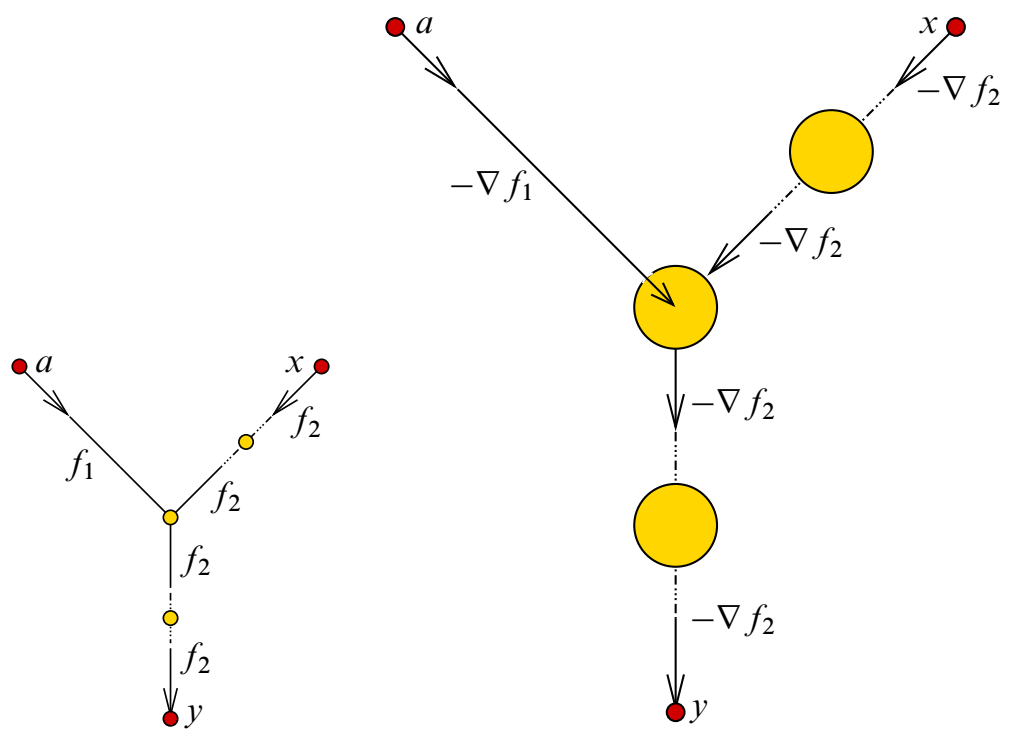

Figure 3: A tree of symbol $(a, x: y)$ on the left and a pearly trajectory corresponding to it on the right

with marked points have symbol $(x: a)$ with $x \in \operatorname{Crit}\left(f_{1}\right), a \in \operatorname{Crit}\left(f_{2}\right)$. The marking is chosen as follows: for all the edges $e$ of type $L, q_{-}(e)=+1, q_{+}(e)=-1$; for the edge $e$ of type $M, q_{-}(e)=0 \in D$ (it is easy to see that the stability condition (iv) in Section 3.1 together with the form of the symbol imply that there can only be a unique edge of type $M)$. The exit rule is $\Theta\left(f_{1} ; L\right)=f_{1}, \Theta\left(f_{1} ; M\right)=f_{2}$ (notice that, this is the first place where the symbol $S$ in the definition of the exit rule at point (v) in Section 3.1 is of use; moreover, because the symbol is $(x: a)$, the only disk type vertex with the exit edge of type $M$ is the one just before the end of the tree). The virtual dimension is in this case $\delta=|x|-|y|+\mu([\mathcal{T}])$ and the quantum inclusion is defined by

$$
i_{L}: \mathcal{C}\left(f_{1}\right) \rightarrow C M\left(f_{2} ; \mathcal{R}\right) ; \quad i_{L}(x)=\sum\left(\#_{2} \mathcal{P}_{\mathcal{T}, Q}\right) a T^{[\mathcal{T}]} .
$$

(e) (Invariance) Assume given two sets of data $\left(f, \rho_{L}, J\right)$ and $\left(f^{\prime}, \rho_{L}^{\prime}, J^{\prime}\right)$ so that the pearl complexes $\mathcal{C}\left(L ; \mathcal{R} ; f, \rho_{L}, J\right)$ and $\mathcal{C}\left(L ; \mathcal{R} ; f^{\prime}, \rho_{L}^{\prime}, J^{\prime}\right)$ are defined. We now need to construct a chain morphism

$$
\phi^{F, \tilde{\rho}_{L}, \widetilde{J}}: \mathcal{C}\left(L ; f, \rho_{L}, J\right) \rightarrow \mathcal{C}\left(L ; f^{\prime}, \rho_{L}^{\prime}, J^{\prime}\right)
$$

which induces a canonical isomorphism in homology (we omit the ring $\mathcal{R}$ from the notation). This morphism is associated to: $\widetilde{J}=\left\{J_{t}\right\}$, a smooth one parametric family of almost complex structures with $J_{0}=J, J_{1}=J^{\prime}, F: L \times[0,1] \rightarrow \mathbb{R}$, a Morse 
homotopy (see Biran and Cornea [12] as well as Cornea and Ranicki [26]) between $f$ and $f^{\prime}, \tilde{\rho}_{L}$ a metric on $L \times[0,1]$ with $\left.\tilde{\rho}\right|_{L \times\{0\}}=\rho_{L}$ and $\left.\tilde{\rho}\right|_{L \times\{1\}}=\rho_{L}^{\prime}$. In other words, we use here a slight modification of our standard construction by taking $\mathcal{F}=\{F\}$ and using trees as at point (a), but with $F$ replacing $f, \tilde{\rho}_{L}$ replacing $\rho_{L}$ and $\widetilde{J}$ instead of $J$. The symbol is $(x: y)$ with $\left.x \in \operatorname{Crit}_{k+1}(F)\right|_{L \times\{0\}}=\operatorname{Crit}_{k}(f)$ and $\left.y \in \operatorname{Crit}_{s}(F)\right|_{L \times\{1\}}=\mathrm{Crit}_{s}\left(f^{\prime}\right)$. In particular, both the marked point selector $Q$ and the exit rule are the same as at point (a). The points (a), (b), (c), in Section 3.1 B are also modified as follows.

The set $S_{\mathcal{T}, Q}$ is now a subset of the product

$$
\Pi(\mathcal{T}, \widetilde{J})=\prod_{v \in v_{\text {int }}(\mathcal{T}), t \in[0,1]} \mathcal{M}_{t}([v], \widetilde{J})
$$

where

$$
\mathcal{M}_{t}([v], \widetilde{J})=\left\{u:(D, \partial D) \rightarrow(M \times\{t\}, L \times\{t\}) \mid \bar{\partial}_{J_{t}}(u)=0\right\} .
$$

The flow $\gamma_{t}^{f}$ is replaced by the negative gradient flow, $\gamma_{t}^{F}$, of $F$ with respect to $\tilde{\rho}_{L}$ (which is a flow on $L \times[0,1]$ ) and points (a), (b), (c) now apply without further modifications. In short, the curves which appear at the start (and respectively the end) of the edge $e$ are $J_{t}$-holomorphic where $t$ is determined by the second coordinate of the starting point (respectively, end) of the flow line of $-\nabla(F)$ which corresponds to $e$. Notice that in our construction all intervening curves are genuinely $J_{t_{0}}$-holomorphic for some $t_{0} \in[0,1]$ in contrast to the continuation method familiar in Floer theory.

The virtual dimension is $\delta=|x|-|y|+\mu[\mathcal{T}]$. The morphism is defined by

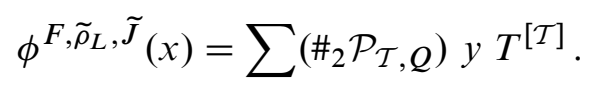

An additional parameter is required to show that the morphism induced in homology is canonical - by constructing a chain homotopy between any two morphisms as above which is associated to a Morse homotopy of Morse homotopies. Perfectly similar constructions provide chain homotopies which proves the invariance of the quantum product and of the module structure.

(f) (The associativity type relations) The purpose here is to define the moduli spaces needed to prove the associativity of the quantum product as well as the other relations at point (ii) of Theorem A.

For the associativity of the quantum product we will use three functions $f_{i}: L \rightarrow$ $\mathbb{R}, i \in\{1,2,3\}$ and the moduli spaces to be considered are modeled on trees $\mathcal{T}$ of symbol $\left(x_{1}, x_{2}, x_{3}: w\right)$ with $x_{i} \in \operatorname{Crit}\left(f_{i}\right)$ and $w \in \operatorname{Crit}\left(f_{3}\right)$; the exit rule is $\Theta\left(f_{k_{1}}, \ldots, f_{k_{i}}\right)=f_{\max \left\{k_{1}, \ldots, k_{i}\right\}}$. We will now define a particular family of marked point selectors $\bar{Q}=\left\{Q_{\theta}\right\}$ consisting of one marked point selector $Q_{\theta}$ for each 
$\theta \in(0,2 \pi / 3)$. This $Q_{\theta}$ is as in the definition of the quantum product for all vertices of valence 2 and 3 and in case one vertex $v$ is of valence 4 then the first two edges arriving at $v$ (in the planar order) and the exit edge are attached at the roots of the unity of order 3 -in the same way as for the vertices of valence 3 . The third arriving edge $e$ satisfies $q_{\theta,+}(e)=e^{i \theta}$. The moduli spaces used to prove the associativity of the quantum product are

$$
\mathcal{P}_{\mathcal{T}, \bar{Q}}=\bigcup_{\theta \in(0,2 \pi / 3)} \mathcal{P}_{\mathcal{T}, Q_{\theta}} \times\{\theta\}
$$

The resulting virtual dimension of this moduli space is $\delta=\left|x_{1}\right|+\left|x_{2}\right|+\left|x_{3}\right|-|w|+$ $\mu[\mathcal{T}]+1$ (the +1 comes from the additional parameter $\theta$ ).

Both $0-$ and 1-dimensional such moduli spaces are needed to verify associativity: the 0 -dimensional moduli spaces are used to define a chain homotopy $\eta: \mathcal{C}\left(f_{1}\right) \otimes_{\mathcal{R}}$ $\mathcal{C}\left(f_{2}\right) \otimes_{\mathcal{R}} \mathcal{C}\left(f_{3}\right) \rightarrow \mathcal{C}\left(f_{3}\right)$ and the 1 -dimensional moduli spaces are used to prove the relation $((-\circ-) \circ-)+(-\circ(-\circ-))=(d \eta+\eta d)(-\otimes-\otimes-)$. More details appear in [12].

To prove the relation $(a * b) \circledast x=a *(b \circledast x)$ with $a, b \in Q H(M ; \mathcal{R})$ and $x \in Q H(L ; \mathcal{R})$ we use two functions $f_{1}, f_{2}: M \rightarrow \mathbb{R}$ and $f_{3}: L \rightarrow \mathbb{R}$. The moduli spaces in question are modeled on trees $\mathcal{T}$ of symbol $(a, b, x: y)$ with $a \in \operatorname{Crit}\left(f_{1}\right), b \in \operatorname{Crit}\left(f_{2}\right)$, $x, y \in \operatorname{Crit}\left(f_{3}\right)$. The exit rule is $\Theta\left(f_{k_{1}}, \ldots, f_{k_{s}}\right)=f_{\max \left\{k_{1}, \ldots, k_{s}\right\}}$. Again we will need to define a special family of marked point selectors, denoted in this case by $\widetilde{Q}=\left\{Q_{\tau}\right\}$ for $\tau \in(-1,0)$. The marked point selector $Q_{\tau}$ is as at point (c) for all vertices of valence 2 or 3 . If a vertex is of valence 4 then the marked points are the same as at point (c) for the edges of type $L$. At this vertex there are also two entering edges of type $M$ and the respective marked points are as follows: for the edge $e_{1}$ colored with $f_{1}$, we put $q_{+}\left(e_{1}\right)=0 \in D^{2}$; for the edge $e_{2}$, colored with $f_{2}$, we put $q_{+}\left(e_{1}\right)=\tau \in(-1,0) \subset \operatorname{Int}\left(D^{2}\right)$. Finally the moduli spaces needed here are

$$
\mathcal{P}_{\mathcal{T}, \tilde{Q}}=\bigcup_{\tau \in(-1,0)} \mathcal{P}_{\mathcal{T}, Q_{\tau}} \times\{\tau\}
$$

We will again need moduli spaces of this sort and of dimensions 0 and 1 . As at point (c), to achieve transversality, some of the disks appearing in these moduli spaces will need to be perturbed by using perturbations as described by Equation (15). More precisely, in the moduli spaces of dimension 0 , if a vertex is of valence 4 , then its corresponding curve is a perturbed $J$-disk. In the moduli spaces of dimension 1 , the disks of valence 3 as well as the disk of valence 4 (if present) need to be perturbed. Again, for more details see our paper [12]. 
(g) (Comparison with Floer homology) The version of Floer homology that we need is defined in the presence of a generic Hamiltonian $H: M \times[0,1] \rightarrow \mathbb{R}$. Consider the path space $\mathcal{P}_{0}(L)=\left\{\gamma \in C^{\infty}([0,1], M) \mid \gamma(0) \in L, \gamma(1) \in L,[\gamma]=1 \in \pi_{2}(M, L)\right\}$ and inside it the set of (contractible) orbits, or chords, $\mathcal{O}_{H} \subset \mathcal{P}_{0}(L)$ of the Hamiltonian flow $X_{H}$. Assuming $H$ to be generic we have that $\mathcal{O}_{H}$ is a finite set. Fix a generic almost complex structure $J$.

There is a natural epimorphism $p: \pi_{1}\left(\mathcal{P}_{0}(L)\right) \rightarrow H_{2}^{D}(M, L)$ and we take $\widetilde{\mathcal{P}}_{0}(L)$ to be the regular, abelian cover associated to $\operatorname{ker}(p)$ so that $H_{2}^{D}(M, L)$ acts as the group of deck transformations for this covering. Consider all the lifts $\tilde{x} \in \widetilde{\mathcal{P}}_{0}(L)$ of the orbits $x \in \mathcal{O}_{H}$ and let $\widetilde{\mathcal{O}}_{H}$ be the set of these lifts. Fix a base point $\eta_{0}$ in $\widetilde{\mathcal{P}}_{0}(L)$ and define the degree of each element $\tilde{x}$ by $|\tilde{x}|=\mu\left(\tilde{x}, \eta_{0}\right)$ with $\mu$ being here the Viterbo-Maslov index. Let $\mathcal{R}$ be a commutative $\mathbb{Z}_{2}\left[H_{2}^{D}(M, L)\right]$-algebra (eg $\mathcal{R}=\Lambda$, or $\Lambda^{\prime}$ or $\mathbb{Z}_{2}\left[H_{2}^{D}(M, L)\right]$ itself but not $\Lambda^{+}$or $\left.\tilde{\Lambda}^{+}\right)$.

The Floer complex is the $\mathcal{R}$-module

$$
C F_{*}(L ; H, J)=\mathbb{Z}_{2}\left\langle\widetilde{\mathcal{O}}_{H}\right\rangle \otimes_{\mathbb{Z}_{2}\left[H_{2}^{D}(M, L)\right]} \mathcal{R} .
$$

The differential is given by $d \tilde{x}=\sum \# \mathcal{M}(\tilde{x}, \tilde{y}) \tilde{y}$ where $\mathcal{M}(\tilde{x}, \tilde{y})$ is the moduli space of solutions $u: \mathbb{R} \times[0,1] \rightarrow M$ of Floer's equation $\partial u / \partial s+J \partial u / \partial t+\nabla H(u, t)=0$ which satisfy $u(\mathbb{R} \times\{0\}) \subset L, u(\mathbb{R} \times\{1\}) \subset L$ and they lift in $\widetilde{\mathcal{P}}_{0}(L)$ to paths relating $\tilde{x}$ and $\tilde{y}$. Moreover, the sum is subject to the condition $\mu(\tilde{x}, \tilde{y})-1=0$.

The comparison map from the pearl complex

$$
\phi_{f, H}: \mathcal{C}\left(L ; f, \rho_{L}, J\right) \rightarrow C F(L ; H, J)
$$

is defined by the PSS method (see Piunikin, Salamon and Schwarz [48] and, in the Lagrangian case, Barraud and Cornea [4], Cornea and Lalonde [24] and Albers [1]) as well as the map in the opposite direction

$$
\psi_{H, f}: C F(L ; H, J) \rightarrow \mathcal{C}\left(L ; f, \rho_{L}, J\right) .
$$

In our language, the map $\phi_{f, H}$ is defined by counting elements in moduli spaces modeled on trees of symbol $(x: \gamma)$ with $x \in \operatorname{Crit}(f), \gamma \in \widetilde{\mathcal{O}}_{H}$ - thus notice a first modification of the "pearl" construction, the exit of the tree is labeled in this case by an orbit. There will be just one Morse function $f: L \rightarrow \mathbb{R}$ and the exit rule as well as the marked point selector are as at point (a) (in Section 3.2). However, the last vertex in the tree, the exit, will no longer correspond to a critical point but rather to a solution $u: \mathbb{R} \times[0,1] \rightarrow M$ of the equation

$$
\partial u / \partial s+J \partial u / \partial t+\beta(s) \nabla H(u, t)=0
$$


so that $\beta: \mathbb{R} \rightarrow[0,1]$ is an appropriate increasing smooth function supported in the interval $[-1,+\infty)$ and which is constant equal to 1 on $[1,+\infty)$. This solution $u$ has also to satisfy $u(\mathbb{R} \times\{0\}) \subset L, u(\mathbb{R} \times\{1\}) \subset L, \lim _{s \rightarrow \infty} u(s,-)=\gamma(-)$ and $\lim _{s \rightarrow-\infty} u(s,-)=P \in L$ so that condition (c) in Section $3.1 \mathrm{~B}$ which describes the geometric relation associated to the exit edge $e$, is replaced by: " $\exists t>0$ so that $\gamma_{t}^{f_{e}}\left(u_{e_{-}}\left(q_{-}(e)\right)\right)=P$ ". The map $\psi_{H, f}$ is given by using similar moduli spaces but with the first vertex being a perturbed one (the perturbation will use the function $\beta^{\prime}=1-\beta$ ) and starting from an element of $\widetilde{\mathcal{O}}_{H}$. Proving that these maps are chain morphisms and that their compositions induce inverse maps in homology depends, in the first instance, on using one-dimensional moduli spaces as above and, in the second, on yet some other moduli spaces which will produce the needed chain homotopies. For $\phi_{f, H} \circ \psi_{H, f}$ these moduli spaces are again modeled on trees with a single entry and exit, as in the differential of the pearl complex, but both the exit and entry vertices are of the perturbed type as in (16) (with a perturbation $\beta^{\prime}$ for the entry and $\beta$ for the exit). In the case of $\psi_{H, f} \circ \phi_{f, H}$ one of the internal vertices satisfies a perturbed equation but a function $\beta^{\prime \prime}$ with support in an interval of type $[-r, r]$ is used instead of $\beta$ (see again Albers [1] and Biran and Cornea [12] for details).

(h) (The augmentation) Fix a pearl complex $\mathcal{C}(L ; \mathcal{R} ; f, \rho, J)$ where $\mathcal{R}$ is a $\tilde{\Lambda}^{+}$ algebra (as in Section 2.1.2). Define

$$
\epsilon_{L}: \mathcal{C}(L ; \mathcal{R} ; f, \rho, J) \rightarrow \mathcal{R}
$$

by $\epsilon_{L}(x)=0$ for all critical points $x \in$ Crit $>0(f)$ and $\epsilon_{L}(x)=1$ for those critical points $x \in \operatorname{Crit}(f)$ with $|x|=0$. Notice that a (local) minimum $x_{0}$ cannot appear in the differential $d y=\sum a_{z, A} z T^{A}$ of any critical point $y$ except for $A=0$ and $|y|=1$. Indeed, a moduli space $\mathcal{P}_{\mathcal{T}}$ modeled on a tree $\mathcal{T}$ of symbol $\left(y: x_{0}\right)$ as at the point (a) in this section is of dimension $|y|-1+\mu[\mathcal{T}]$ and thus can only be of dimension 0 if $[\mathcal{T}]=0$. Since for each critical point of index 1 there are precisely two flow lines emanating from it, we deduce that $\epsilon_{L} \circ d=0$ and so $\epsilon_{L}$ is a chain map. The same type of argument, now applied to the comparison map constructed in the invariance argument at point (e) shows that, in homology, $\epsilon_{L}$ commutes with the canonical isomorphisms.

\subsection{Transversality}

As mentioned before we will not give here the full proof of transversality (we refer to [12] for that). However, we will review the main ideas.

Given an $\mathcal{F}$-colored tree with marked points $(\mathcal{T}, Q)$ as defined in Section 3.1 we discuss the proof of the fact that, for generic $J$, the associated moduli space $\mathcal{P}_{\mathcal{T}, Q}$ is a 
manifold of dimension equal to the virtual dimension $\delta(\mathcal{T})$. The finite family $\mathcal{F}$ of Morse functions defined on $L$ or on $M$ is fixed throughout the section and it contains at most three functions defined on $L$ and two defined on $M$. The only moduli spaces to be treated are those appearing in Section 3.2.

In the argument, slightly more general such moduli spaces will also be needed. As before, the numbers of entries will always be at most 3 and there will be a single exit. However, we will not impose any particular restriction on the exit rule (in particular, all possible exit rules will be allowed in the inductive argument below). Secondly, we will need to prove the regularity of moduli spaces of type

$$
\mathcal{P}_{\mathcal{T}, Q}=\bigcup_{s \in U} \mathcal{P}_{\mathcal{T}}, Q_{s} \times\{s\}
$$

where $Q=\left\{Q_{s}\right\}_{s \in U}$ is a family of marked point selectors $Q_{s}$ so that at most two of the marked points provided by $Q_{s}$ (and which are associated to vertices of valence at least 3 ) are allowed to take the values in the set $U$. Here $U=U_{1} \times U_{2}$ where both $U_{i} \subset D$ are connected submanifolds without boundary of dimension at most 2 . These types of moduli spaces have already appeared in the discussion of associativity at the point (f). in Section 3.2 and some additional ones will appear in the transversality argument. More precisely, our allowed choices for these sets $U_{i}$ are as follows. If $\operatorname{dim} U_{i}=0$, then $U_{i}$ coincides with one of the marked points appearing in the description of the marked point selectors in Section 3.2 (in other words, $U_{i}$ is one of the points $\left.+1,-1, e^{2 \pi i / 3}, e^{4 \pi i / 3}, 0 \in D\right)$; if $\operatorname{dim} U_{i}=1$, then $U_{i}$ is one of the following two choices $(-1,0) \subset D$ or $\left\{e^{i t}\right\}_{0 \leq t \leq 2 \pi / 3} \subset \partial D$ (both have been already used at point (f) in Section 3.2); finally, if $\operatorname{dim} U_{i}=2$, then $U_{i}=\operatorname{Int}(D)$. We will still refer to these moduli spaces by $\mathcal{P}_{\mathcal{T}, Q}$ and refer to them as $\mathcal{F}$-colored moduli spaces with marked points and, by a slight abuse of notation, $Q$ will still be referred to as a marked point selector. The virtual dimension of these moduli spaces is given by a formula similar to (10) to which is added another term depending on the dimension of the sets $U_{i}$ as above and on the valence of the vertices to which these marked points are associated. In view of this, we denote this virtual dimension by $\delta(\mathcal{T}, Q)$.

Let $\mathcal{P}_{\mathcal{T}, Q}^{*}$ be the moduli spaces associated to $\mathcal{F}$-colored trees with marked points $(\mathcal{T}, Q)$ which satisfy the additional condition that all the $J$-holomorphic curves $u_{v}$ corresponding to the internal vertexes $v \in v(\mathcal{T})$ have the property that they are simple and that they are absolutely distinct. We recall that a curve $u: \Sigma \rightarrow M$ is simple if it is injective at almost all points $z \in \operatorname{Int}(\Sigma)$ in the sense that $d u_{z} \neq 0$ and $u^{-1}(u(z))=\{z\}$. The curves $\left(u_{v}\right)$ are absolutely distinct if no single curve $u_{v}$ has its image included in the union of the images of the others, $\operatorname{Im}\left(u_{v}\right) \not \subset \cup_{v^{\prime} \in v(\mathcal{T}) \backslash\{v\}} \operatorname{Im}\left(u_{v^{\prime}}\right)$. By a straightforward adaptation of now standard techniques, as in [40] Chapter 3 in 
particular Proposition 3.4.2, we obtain that $\mathcal{P}_{\mathcal{T}, Q}^{*}$ is a manifold of dimension $\delta(\mathcal{T}, Q)$, in general noncompact, with a boundary consisting of configurations so that some edges in $\mathcal{T}$ are represented by gradient flow lines of 0 -length (recall that we allow the length of edges to be $\geq 0$ ). Notice that, in case some perturbed $J$-holomorphic curves appear also in the elements of $\mathcal{P}_{\mathcal{T}, Q}$ as at (c) in Section 3.2, there is no need to impose any similar condition to them: a choice of generic perturbations insures the needed transversality. To simplify the argument, we focus in the proof below on the case where just a single almost complex structure appears in the definition of our moduli spaces. However, if as for the invariance argument, point (e) in Section 3.2, we need to deal with a family $\widetilde{J}=\left\{J_{t}\right\}_{t \in[0,1]}$ of almost complex structures, then the "absolutely distinct" condition only needs to be verified for the disks that are $J_{t}$-holomorphic for each $t$ at a time and by taking this remark into account the argument below adapts easily to this setting.

The key point is to show that $\mathcal{P}_{\mathcal{T}, Q}^{*}=\mathcal{P}_{\mathcal{T}, Q}$ as long as $\delta(\mathcal{T}, Q) \leq 1$. In turn, the proof of this is by induction. To be more explicit, fix the $\operatorname{symbol} \operatorname{symb}(\mathcal{T})=\left(x_{1}, x_{2}, \ldots, x_{l}: y\right)$ of the tree $\mathcal{T}$. Fix some $k \in \mathbb{N}$. The combinatorial data used to define $\mathcal{F}$-colored trees with marked points $(\mathcal{T}, Q)$ so that $\mu[\mathcal{T}] \leq k$ is finite. Thus, up to isomorphism, there are only finitely many such trees. Suppose, by induction, that for all $\mathcal{F}$-colored trees with marked points $\left(\mathcal{T}^{\prime}, Q^{\prime}\right)$ of symbol of length at most 4 and with $\mu\left[\mathcal{T}^{\prime}\right]<\mu[\mathcal{T}]$ and $\delta\left(\mathcal{T}^{\prime}, Q^{\prime}\right) \leq 1$, we have

$$
\mathcal{P}_{\mathcal{T}^{\prime}, Q^{\prime}}^{*}=\mathcal{P}_{\mathcal{T}^{\prime}, Q^{\prime}}
$$

To prove identity (17) for $\mathcal{T}$ it suffices to show that the following simplification step is true:

$$
\mathcal{P}_{\mathcal{T}, Q} \neq \mathcal{P}_{\mathcal{T}, Q}^{*} \Rightarrow\left\{\begin{array}{l}
\exists\left(\mathcal{T}^{\prime}, Q^{\prime}\right) \text { such that } \\
\operatorname{symb}\left(\mathcal{T}^{\prime}\right)=\operatorname{symb}(\mathcal{T}), \mu\left(\left[\mathcal{T}^{\prime}\right]\right)<\mu([\mathcal{T}]), \\
\delta\left(\mathcal{T}^{\prime}, Q^{\prime}\right)<0, \mathcal{P}_{\mathcal{T}^{\prime}, Q^{\prime}} \neq \varnothing .
\end{array}\right.
$$

Indeed, if $\delta\left(\mathcal{T}^{\prime}, Q^{\prime}\right)<0$, the identity (17) together with the regularity of the moduli spaces consisting of simple, absolutely distinct curves implies that $\mathcal{P}_{\mathcal{T}^{\prime}, Q^{\prime}}=\varnothing$ and the conclusion follows by contradiction.

The key to prove (18) is a structural result concerning $J$-holomorphic disks which is the disk counterpart of the multiply-covered $\leftrightarrow$ almost everywhere injective dichotomy valid in the case of $J$-holomorphic spheres. One such result is due to Lazzarini [ 37 ; 38] (an alternative one is due to Kwon and Oh [36]). Here are more details on this point. 
Let $u:(D, \partial D) \rightarrow(M, L)$ be a nonconstant $J$-holomorphic disk. Put $\mathcal{C}(u)=$ $u^{-1}(\{d u=0\})$. Define a relation $\mathcal{R}_{u}$ on pairs of points $z_{1}, z_{2} \in \operatorname{Int} D \backslash \mathcal{C}(u)$ in the following way:

$$
z_{1} \mathcal{R}_{u} z_{2} \Longleftrightarrow\left\{\begin{array}{l}
\forall \text { neighborhoods } V_{1}, V_{2} \text { of } z_{1}, z_{2}, \\
\exists \text { neighborhoods } U_{1}, U_{2} \text { such that: } \\
\text { (i) } z_{1} \in U_{1} \subset V_{1}, z_{2} \in U_{2} \subset V_{2} . \\
\text { (ii) } u\left(U_{1}\right)=u\left(U_{2}\right) .
\end{array}\right.
$$

Denote by $\overline{\mathcal{R}}_{u}$ the closure of $\mathcal{R}_{u}$ in $D \times D$. Note that $\overline{\mathcal{R}}_{u}$ is reflexive and symmetric but it may fail to be transitive (see Lazzarini [37] for more details on this). Define the noninjectivity graph of $u$ to be:

$$
\mathcal{G}(u)=\left\{z \in D \mid \exists z^{\prime} \in \partial D \text { such that } z \overline{\mathcal{R}}_{u} z^{\prime}\right\} .
$$

It is proved in $[37 ; 38]$ that $\mathcal{G}(u)$ is indeed a graph (with a finite number of branching points) and its complement $D \backslash \mathcal{G}(u)$ has finitely many connected components. We use the following theorem due to Lazzarini (see his paper [37] as well as [38]).

Theorem 3.3.1 (Decomposition of disks [37; 38]) Let $u:(D, \partial D) \rightarrow(M, L)$ be a nonconstant $J$-holomorphic disk. Then for every connected component $\mathfrak{D} \subset D \backslash \mathcal{G}(u)$, there is a compact Riemann surface with boundary $\left(S_{\mathfrak{D}}, \partial S_{\mathfrak{D}}\right)$, a complex embedding $h_{\mathfrak{D}}:\left(S_{\mathfrak{D}}, \partial S_{\mathfrak{D}}\right) \rightarrow(D, \mathcal{G}(u))$ whose interior verifies $h_{\mathfrak{D}}\left(\operatorname{Int} S_{\mathfrak{D}}\right)=\mathfrak{D}$, a simple $J-$ holomorphic disk $v_{\mathfrak{D}}:\left(D^{\prime}, \partial D^{\prime}\right) \rightarrow(M, L)$, and a surjective map $\pi_{\mathfrak{D}}: S_{\mathfrak{D}} \rightarrow D^{\prime}$, holomorphic on Int $S_{\mathfrak{D}}$ and continuous on $S_{\mathfrak{D}}$ of well defined degree $m_{\mathfrak{D}} \in \mathbb{N}$, such that the following holds: $v_{\mathfrak{D}} \circ \pi_{\mathfrak{D}}=u \circ h_{\mathfrak{D}}$. Moreover, in $H_{2}^{D}(M, L ; \mathbb{Z})$ we have

$$
[u]=\sum_{\mathfrak{D}} m_{\mathfrak{D}}\left[v_{\mathfrak{D}}\right]
$$

where the sum is taken over all connected components $\mathfrak{D} \subset D \backslash \mathcal{G}(u)$.

The notion of a complex embedding just mentioned is taken from [37]. It is defined as follows. Let $(S, \partial S)$ be a compact Riemann surface with boundary and $\mathcal{G} \subset D$ an embedded graph (see Lazzarini [37; 38]). A complex embedding $h:(S, \partial S) \rightarrow(D, \mathcal{G})$ is a holomorphic map with the properties that $h(\operatorname{Int} S) \cap \mathcal{G}=\varnothing$ and $h^{-1}(h(z))=\{z\}$ for every $z \in \operatorname{Int} S$. (Thus $h$ need not be injective along $\partial S$.)

Two Lemmas, 3.3.2 and 3.3.3, to be stated a bit later, are easy consequences of the theorem above and, as we will see, they reduce our problem to a sequence of combinatorial verifications. 
Returning to the proof of (18) we proceed in two steps. First we discuss the argument insuring that all $J$-curves involved are simple. The second step will show that they can also be assumed to be absolutely distinct. We focus here on the case $\operatorname{dim}(L) \geq 3$ and will comment on the case $\operatorname{dim}(L) \leq 2$ at the end.

Thus, suppose that $u \in \mathcal{P}_{\mathcal{T}, Q}$ is so that $u=\left(u_{v}\right)_{v \in v_{\text {int }}(\mathcal{T})}$ and for some internal vertex $v \in v(\mathcal{T})$ the corresponding $J$-holomorphic curve $u_{v}$ is not simple.

In the trees used in this paper a sphere-type vertex does not carry more than three incidence points. Therefore, in case $u_{v}$ is a $J$-sphere it can clearly be replaced by a simple one $u_{v}^{\prime}$ and the marked point selector is not modified. This means that we may take in this case $\mathcal{T}^{\prime}$ to be topologically the same tree as $\mathcal{T}$ except that the label of the vertex $v$ is now $\left[u_{v}^{\prime}\right]$ instead of $\left[u_{v}\right]$. Thus we may now suppose that $u_{v}$ is a $J$-disk. To deal with this case we will make use of the following consequence of Theorem 3.3.1. We refer to [12] for the proof.

Lemma 3.3.2 Suppose $n=\operatorname{dim} L \geq 3$. Then there exists a second category subset $\mathcal{J}_{\text {reg }} \subset \mathcal{J}(M, \omega)$ such that for every $J \in \mathcal{J}_{\text {reg }}$ the following holds. For every nonconstant, nonsimple $J$-holomorphic disk $u:(D, \partial D) \rightarrow(M, L)$ there exists a $J$-holomorphic disk $u^{\prime}:(D, \partial D) \rightarrow(M, L)$ with the following properties:

(1) $u^{\prime}(D)=u(D)$ and $u^{\prime}(\partial D)=u(\partial D)$.

(2) $u^{\prime}$ is simple.

(3) $\omega\left(\left[u^{\prime}\right]\right)<\omega([u])$. In particular, if $L$ is monotone we also have $\mu\left(\left[u^{\prime}\right]\right)<\mu([u])$.

We apply Lemma 3.3.2 to replace the $J$-disk $u_{v}$ by the simple disk $u_{v}^{\prime}$ provided by the Lemma. Thus, to prove (18), the relevant tree $\mathcal{T}^{\prime}$ that we are looking for is identified with $\mathcal{T}$ except that the vertex $v$ will now be labeled by $\left[u_{v}^{\prime}\right]$. A slightly delicate point needs to be made concerning the marked point selector $Q^{\prime}$ corresponding to $\mathcal{T}^{\prime}$. The way this is constructed is the following: as $u_{v}^{\prime}(D)=u_{v}(D)$, and $u_{v}^{\prime}(\partial D)=u_{v}(\partial D)$, the points $u_{v}\left(q_{ \pm}(e)\right.$ ) (where $e$ is an incident edge at $v$ ) can be lifted to the domain of $u_{v}^{\prime}$ and used as marked points there. Of course, this works only if all these points, $u_{v}\left(q_{ \pm}(e)\right)$, are distinct. If this is not the case some additional vertices need to be included in the tree so that they correspond to constant disks or spheres which are related to the vertex $v$ by edges colored by functions in $\mathcal{F}$ and of 0 -length.

We still need to verify that $\delta\left(\mathcal{T}^{\prime}, Q^{\prime}\right)<0$. Given that $N_{L} \geq 2$ and so $\mu\left(u_{v}^{\prime}\right)<\mu\left(u_{v}\right)-1$ this inequality is automatic if $Q^{\prime}=Q$ because in this case $\delta\left(\mathcal{T}^{\prime}, Q^{\prime}\right) \leq \delta(\mathcal{T}, Q)-N_{L}$. This is the case if $v$ carries two or three marked points all on $\partial D$. The same is true also if $v$ carries two marked points, one on the boundary and one in the interior of $D$. Suppose now that $v$ carries two boundary marked points, -1 and +1 , and the interior 
marked point 0 (as at point (c) in Section 3.2). In this case the marked point selector for $\mathcal{T}^{\prime}$ cannot be assumed to be the same as that for $\mathcal{T}$ : the internal marked point for $u_{v^{\prime}}$ cannot be assumed anymore to be as assigned by $Q$ but can be anywhere inside $D$ - in other words in this case $Q^{\prime}=\left\{Q_{s}\right\}_{s \in \operatorname{Int}(D)}$. In this situation we have $\mu\left[\mathcal{T}^{\prime}\right] \leq \mu[\mathcal{T}]-N_{L}$ and it is easy to see that $\delta\left(\mathcal{T}^{\prime}, Q^{\prime}\right) \leq \delta(\mathcal{T}, Q)-N_{L}+1$. Thus, if $\delta(\mathcal{T}, Q)=0$ we still have $\delta\left(\mathcal{T}^{\prime}, Q^{\prime}\right)<0$ so that $(17)$ remains true for the moduli spaces needed to define the module structure without the need to use any perturbations. However, to prove the fact that the operation defined there is a chain morphism we need to use moduli spaces as before but which satisfy $\delta(\mathcal{T}, Q)=1$. This is precisely why we use perturbed $J$-holomorphic disks in this case: as mentioned before, the proof of the transversality of the relevant evaluation maps requires only the nonperturbed $J$-holomorphic curves to be simple and absolutely distinct. The same issue appears for both the 0 - and 1-dimensional moduli spaces used to prove the associativity of the module action as in the second part of point (f) in Section 3.2 and this shows that the perturbations indicated there are necessary. Full details for these arguments are found in [12].

We now pass to the second step: showing that the $J$-curves $\left\{u_{v}\right\}_{v}$ are absolutely distinct. The main tool is the next result which can be deduced too from Theorem 3.3.1.

Lemma 3.3.3 Suppose $n=\operatorname{dim} L \geq 3$. Then there exists a second category subset $\mathcal{J}_{\text {reg }} \subset \mathcal{J}(M, \omega)$ such that for every $J \in \mathcal{J}_{\text {reg }}$ the following holds. If $u, w:(D, \partial D) \rightarrow$ $(M, L)$ are simple $J$-holomorphic disks such that $u(D) \cap w(D)$ is an infinite set, then at least one of the following relations is valid:

- $u(D) \subset w(D)$ and $u(\partial D) \subset w(\partial D)$.

- $w(D) \subset u(D)$ and $w(\partial D) \subset u(\partial D)$.

This implies that if the $J$-curves in $\left\{u_{v}\right\}_{v \in v_{\text {int }}(\mathcal{T})}$ are not absolutely distinct, then there exist two vertices $v_{0}$ and $v_{1}$ both corresponding to $J$-holomorphic (unperturbed) curves so that $u_{v_{1}}(D) \subset u_{v_{0}}(D)$ and $u_{v_{1}}(\partial D) \subset u_{v_{0}}(\partial D)$. The aim now is to show that we can "simplify" both $\left\{u_{v}\right\}_{v}$ and the tree by eliminating $v_{1}$ (as well as possibly other vertices and edges) and thus produce a new tree $\left(\mathcal{T}^{\prime}, Q^{\prime}\right)$ of lower Maslov number and with $\delta\left(\mathcal{T}^{\prime}, Q^{\prime}\right)<0$ as well as a new element $\left\{u_{v}^{\prime}\right\}_{v \in v_{\text {int }}}\left(\mathcal{T}^{\prime}\right) \in \mathcal{P}_{\left(\mathcal{T}^{\prime}, Q^{\prime}\right)}$ thus arriving at a contradiction.

There are three different cases to consider:

(i) $v_{0}$ and $v_{1}$ are independent, in the sense that they are on different branches of the tree. 
(ii) $v_{0}$ is above $v_{1}$ in the tree, in the sense that by following the tree starting from $v_{0}$ we reach $v_{1}$.

(iii) $v_{1}$ is above $v_{0}$ in the tree.

In the first two cases we obtain the new tree $\mathcal{T}^{\prime}$ by simply taking the branch in the tree above $v_{0}$ but containing $v_{0}$ and pasting it in $\mathcal{T}$ with $v_{0}$ in the place of $v_{1}$. Thus in the tree $\mathcal{T}^{\prime}$ the vertex $v_{1}$ has disappeared and has been replaced with $v_{0}$. To avoid confusion we denote this vertex in $\mathcal{T}^{\prime}$ by $\widehat{v}_{1}$. The corresponding pearly element $\left\{u_{v}^{\prime}\right\}_{v \in v_{\text {int }}\left(\mathcal{T}^{\prime}\right)}$ will satisfy $u_{v}^{\prime}=u_{v}$ for every $v \neq \widehat{v}_{1}$ and $u_{\widehat{v}_{1}}^{\prime}=u_{v_{0}}$. A similar construction can be performed in the third case. Here is a more precise description of this operation in each of the cases (i)-(iii).

In case (i) we first remove from $\mathcal{T}$ the branch $B_{v_{0}}$ of the tree lying above $v_{0}$ (and including $v_{0}$ ). Then we also remove from $\mathcal{T}$ the path going from $v_{0}$ to the branch point below $v_{0}$ which is closest to $v_{0}$. Denote the remaining tree by $\mathcal{T}_{0}$. We define $\mathcal{T}^{\prime}$ by gluing $B_{v_{0}}$ to $\mathcal{T}_{0}$ identifying $v_{0}$ with $v_{1}$. This new vertex will be now denoted by $\widehat{v}_{1}$. We label $\widehat{v}_{1}$ by the homology class of $v_{0}$ and we define $Q^{\prime}$ at $\widehat{v}_{1}$ using the marked points of both $v_{0}$ and $v_{1}$ except of the exit marked point of $v_{0}$ which becomes irrelevant now and is hence dropped. See Figure 4 for an example.
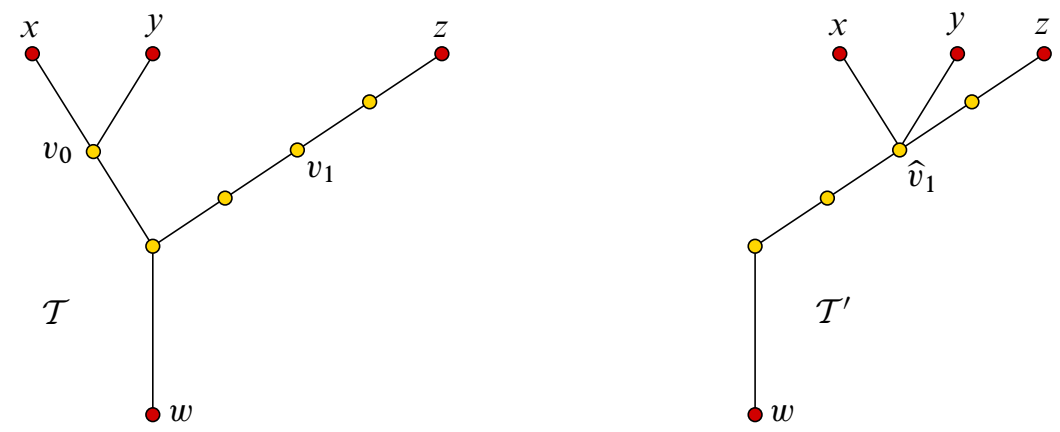

Figure 4: Passing from $\mathcal{T}$ to $\mathcal{T}^{\prime}$ - case (i)

In case (ii), if there is a branch point $\widetilde{v}_{0,1}$ between $v_{0}$ and $v_{1}$ we define $\mathcal{T}^{\prime}$ as follows. We delete from $\mathcal{T}$ the branch $B_{v_{0}}$ as in case (i) above. We also delete from $\mathcal{T}$ the path between $v_{0}$ and $\widetilde{v}_{0,1}$ and denote the remaining tree by $\mathcal{T}_{0}$. We define $\mathcal{T}^{\prime}$ as in case (i) by gluing $B_{v_{0}}$ to $\mathcal{T}_{0}$ identifying $v_{0}$ with $v_{1}$, calling the this new vertex $\hat{v}_{1}$. As in case (i) above, we label $\hat{v}_{1}$ by the homology class of $v_{0}$ and define $Q^{\prime}$ using the marked points of both $v_{0}$ and $v_{1}$, excluding the exit marked point of $v_{0}$. See Figure 5 for an example. To conclude case (ii) we need to describe $\mathcal{T}^{\prime}$ in case there is no branch 

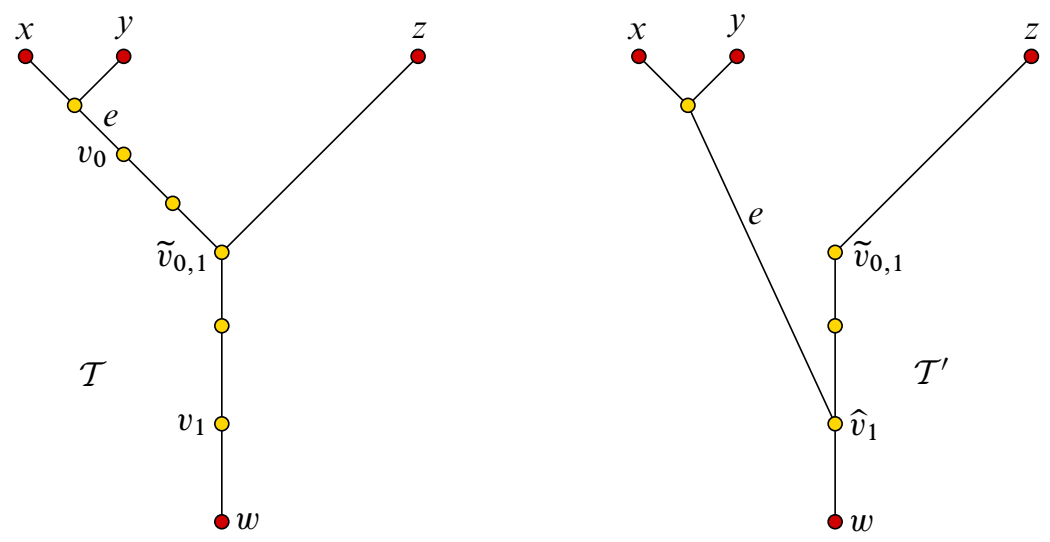

Figure 5: Passing from $\mathcal{T}$ to $\mathcal{T}^{\prime}-$ case (ii)

point between $v_{0}$ and $v_{1}$. In that case, we just define $\mathcal{T}^{\prime}$ by removing the path between $v_{0}$ and $v_{1}$ and identifying $v_{0}$ with $v_{1}$. This new vertex $\widehat{v}_{1}$ is labeled by the label of $v_{0}$ and the marked points now are inherited from $v_{0}$ and $v_{1}$ except of the exiting marked point of $v_{0}$ and the corresponding entering marked point of $v_{1}$ which are now dropped.

Suppose we are now in case (iii), ie $v_{0}$ is lower than $v_{1}$ in the tree. This case is dealt with similarly to (ii). In this case, the tree $\mathcal{T}^{\prime}$ is obtained as before but with the roles of the vertices $v_{0}$ and $v_{1}$ reversed: the branch above $v_{1}$ and containing $v_{1}$ is grafted to the tree in the place of $v_{0}$ and the branch leaving from $v_{1}$ and reaching the first branch point separating $v_{1}$ and $v_{0}$ (or the portion in the tree between $v_{1}$ and $v_{0}$ if no such branch point exists) is omitted. The new vertex (corresponding to $v_{0}$ and $v_{1}$ ) is now called $\hat{v}_{0}$. Again the $J$-curves associated to the vertices of $\mathcal{T}^{\prime}$ are the same as the corresponding curves associated to the vertices of $\mathcal{T}$ except that $u_{\widehat{v}_{0}}=u_{v_{0}}$.

There is yet another point at which care should be taken (in all cases (i)-(iii). It may happen that some of the relevant marked points of $v_{0}$ and of $v_{1}$ coincide (again, we disregard those marked points that are dropped as above), and in this case the description given above for $\left(\mathcal{T}^{\prime}, Q^{\prime}\right)$ is incomplete. If such a coincidence of marked points occurs we need to insert some additional vertices, corresponding to constant $J$-curves, carrying distinguished marked points as well as connecting edges. This modification is straightforward and we will not go into more detail about it.

It now easily follows that the resulting tree $\mathcal{T}^{\prime}$ has a strictly lower Maslov index than $\mathcal{T}$. The dimension verification is also immediate except if $v_{1}$ carries some internal marked points. If there is a single such marked point and $\delta(\mathcal{T}, Q)=0$, then we take $Q^{\prime}=\left\{Q_{s}\right\}_{s \in \text { Int } D}$ (because the internal marked point may now take any value inside $D$ ) and we still have, as in the reduction to simple disks, $\delta\left(\mathcal{T}^{\prime}, Q^{\prime}\right)<0$. If $v_{1}$ 
carries two internal incidence points or if it carries one but $\delta(\mathcal{T}, Q)=1$, then, by the particular choice of the moduli spaces in Section 3.2, $v_{1}$ corresponds to a perturbed $J$-holomorphic disk in contradiction to our starting assumption.

The case $n \leq 2$ is easily reduced to a number of combinatorial problems. The assumptions $n \leq 2, N_{L} \geq 2$ and $\delta(\mathcal{T}, Q) \leq 1$ imply that the total number of $J$-curves is relatively small (for example, there are at most two for the verifications involving the pearl complex) so that combinatorial arguments apply in many of these cases. In fact, it is not hard to use directly Theorem 3.3.1 to deal with trees $\mathcal{T}$ in which the total Maslov index of the vertices represented by $J$-disks is at most 6 (even if there might be additional vertices corresponding to perturbed disks). This covers all the verifications involved with the pearl complex and its invariance, the product and its associativity and invariance, the definition of the module structure and its invariance. This also works for the proof of the relation $(a * b) \circledast x=a \circledast(b \circledast x), a, b \in Q H(M ; \mathcal{R}), x \in Q H(L ; \mathcal{R})$ for $N_{L} \geq 3$. Finally, the remaining case can also be dealt with combinatorially.

\subsection{Compactness and the final step}

The transversality arguments in the previous section show that our moduli spaces are manifolds. We will start here by describing the structure of the compactification of these moduli spaces. For this, besides the transversality results described before, we only need the Gromov compactness theorem (for disks see Frauenfelder [31]). We first remark that given an $\mathcal{F}$-colored tree with marked points $(\mathcal{T}, Q)$ and an associated moduli space $\mathcal{P}_{\mathcal{T}, Q}$ - constructed as described in Section 3.1 - there is a natural Gromov type topology on $\mathcal{P}_{\mathcal{T}}$ as well as a natural compactification $\overline{\mathcal{P}}_{\mathcal{T}}$.

In short, the elements of $\bar{P}_{\mathcal{T}} \backslash P_{\mathcal{T}}$ are modeled on the tree $\mathcal{T}$ and the only modification with respect to our definition in Section 3.1 concerns the points (a), (b), (c), at the end of that section. Specifically, the product $\Pi(\mathcal{T})$ is replaced by its compactification

$$
\bar{\Pi}(\mathcal{T})=\prod_{v \in v_{\text {int }}(\mathcal{T})} \overline{\mathcal{M}}([v], J)
$$

where $\overline{\mathcal{M}}([v], J)$ is the Gromov compactification of $\mathcal{M}([v], J)$ so that, for each internal vertex $v$, the associated geometric object $u_{v} \in \overline{\mathcal{M}}([v], J)$. The points (a), (b), (c), are then replaced by the following variants:

- For each internal edge $e \in e(\mathcal{T})$, the points $u_{e_{-}}\left(q_{-}(e)\right)$ and $u_{e_{+}}\left(q_{+}(e)\right)$ are related by a possibly broken flow line (possibly of 0 -length) of $\gamma^{f_{e}}$.

- For an entry edge, $e$, let $x_{i}$ be the critical point labeling the vertex $e_{-}$. The point $x_{i}$ is related to the point $u_{e_{+}}\left(q_{+}(e)\right)$ by a possibly broken flow line (possibly of 0 -length) of $\gamma f_{e}$. 
- For the exit edge $e$ so that the vertex $e_{+}$is labeled by the critical point $y$ of $f_{e}$, the point $u_{e_{-}}\left(q_{-}(e)\right)$ is related to $y$ by a possibly broken flow line (possibly of 0 -length) of $\gamma^{f_{e}}$.

A remark is needed concerning the marked point selectors. The various marked points which correspond to the same vertex in the configurations described above are again required to be distinct and are given in the same way as that described in Section 3.2. In particular, each time two (or more) such incidence points "merge" a ghost curve needs to be introduced.

From now on we will only focus on $\mathcal{F}$-colored trees that are of virtual dimension $\delta(\mathcal{T}) \leq 1$ and, in view of our transversality results, we may assume that (17) is satisfied so that $\mathcal{P}_{\mathcal{T}}^{*}=\mathcal{P}_{\mathcal{T}}$ (the role of the marked point selector is less crucial in this part and we will omit it from the notation). Under this hypothesis, the first key remark is that each element $\bar{u} \in \overline{\mathcal{P}}_{\mathcal{T}} \backslash \mathcal{P}_{\mathcal{T}}$ contains exactly one configuration among the three types below:

- a flow line broken exactly once,

- a vertex $v_{\bar{u}} \in v(\mathcal{T})$ corresponding to a cusp curve with precisely two components (which can be ghosts),

- a flow line of length 0 .

The reason for this is that if more than a single such configuration occurs we can extract from $\bar{u}$ an object $u^{\prime} \in \mathcal{P}_{\mathcal{T}^{\prime}}$ with $\delta\left(\mathcal{T}^{\prime}\right)<0$ which is impossible because such a moduli space of negative virtual dimension is regular and thus void.

The second important remark is that the condition $N_{L} \geq 2$ insures that no "lateral" bubbling is possible. More explicitly, this means that if the element $\bar{u}$ satisfies condition (ii), then the incidence points associated to the vertex $v_{u}$ are distributed among the two components of the cusp curve so that not all of them are in just one component. This happens because, otherwise, the component which does not carry any of these incidence points can be omitted thus giving rise to an object $u^{\prime}$ which belongs to a moduli space of virtual dimension lower by at least $N_{L}$ than $\delta\left(\mathcal{P}_{\mathcal{T}}\right)$ which again is not possible.

The last step is to use the description of the compactification given above to verify the various relations required to establish the theorem (as described at the points (a) $-(\mathrm{g})$ and (i) in Section 3.2). The technical ingredient for this verification is gluing. Gluing procedures have already appeared for example in [33] and for full details we refer again to [12]. This gluing procedure insures that, when $\delta(\mathcal{T})=1$, each element $\bar{u}$ which is 
modeled on the tree $\mathcal{T}$ and which satisfies exactly one of the properties (i), (ii), (iii) above actually belongs to $\overline{\mathcal{P}}_{\mathcal{T}} \backslash \mathcal{P}_{\mathcal{T}}$ and appears as a boundary element of $\overline{\mathcal{P}}_{\mathcal{T}}$.

Finally, the verification of the relations mentioned involves in an essential way the fact that our algebraic operations are defined by using $\Theta$-admissible trees. The role of the exit rule $\Theta$ (as described at point (v) in Section 3.1) is as follows: for a tree $\mathcal{T}$ with $\delta(\mathcal{T})=1$, if $\bar{u} \in \partial \overline{\mathcal{P}}_{\mathcal{T}}$ satisfies (ii) above, then, due to the fact that "lateral" bubbling is not possible, $\bar{u}$ is also an element of $\partial \overline{\mathcal{P}}_{\mathcal{T}^{\prime}}$ where $\mathcal{T}^{\prime}$ is the tree obtained from $\mathcal{T}$ by replacing the vertex $v_{\bar{u}}$ by two vertices (corresponding to the two components of the cusp curve associated to $v_{\bar{u}}$ ) related by an edge of length 0 whose type is uniquely determined by the exit rule. Moreover, by gluing, each $\bar{u} \in \partial \overline{\mathcal{P}}_{\mathcal{T}}$ satisfying (iii) is an element in the boundary of a moduli space modeled on a tree obtained from $\mathcal{T}$ by replacing the two vertices related by the edge of $0-$ length by a single vertex. Denote by $\partial_{1}\left(\overline{\mathcal{P}}_{\mathcal{T}}\right)$ the parts of the boundary of $\overline{\mathcal{P}}_{\mathcal{T}}$ formed by the points satisfying (i). and fix the symbol $\left(x_{1}, x_{2}, \ldots x_{l}: y\right)$ of $\mathcal{T}$. When summing over all trees (of virtual dimension 1 ) and of fixed symbol we see that the configurations of types (ii) and (iii) cancel (as we will see below, due to the presence of perturbations in some of our moduli spaces, an additional argument is sometimes needed at this point) and so we deduce

$$
\sum_{\operatorname{symb}\left(\mathcal{T}^{\prime}\right)=\left(x_{1}, \ldots x_{l}: y\right)} \# \partial_{1}\left(\overline{\mathcal{P}}_{\mathcal{T}^{\prime}}\right)=0 .
$$

The relations that need to be justified are then obtained by identifying each element $\bar{u} \in \partial_{1}\left(\mathcal{P}_{\mathcal{T}}\right)$ of type (i) with precisely one element of the product $\mathcal{P}_{\mathcal{T}_{1}} \times \mathcal{P}_{\mathcal{T}_{2}}$ where $\mathcal{T}_{1}$ and $\mathcal{T}_{2}$ are the two trees obtained as follows: first, introduce in $\mathcal{T}$ an additional vertex $\widetilde{v}$ on the edge which corresponds to the broken flow line and then let $\mathcal{T}_{1}, \mathcal{T}_{2}$ be the two (sub)-trees which have in common only the vertex $\widetilde{v}$ and whose union gives $\mathcal{T}$.

Clearly, in what concerns the comparison with Floer homology - point (g) in Section 3.2 - the argument above needs to be modified slightly. The required modification is however obvious and we will not discuss it further. However, a more substantial addition to the argument is needed in the case of the perturbations of type (15) which were introduced in the moduli spaces needed to verify that the module action is a chain map and to check some of the related associativity - as at points (c) and (f) in Section 3.2. This happens in precisely two cases. The first - concerning the fact that the module operation is a chain map - has to do with the identification of an element $\bar{u} \in \partial_{1}\left(\mathcal{P}_{\mathcal{T}}\right)$ with an element of the product $\mathcal{P}_{\mathcal{T}_{1}} \times \mathcal{P}_{\mathcal{T}_{2}}$. The problem here is that, by the definition of the relevant 1-dimensional spaces at the end of (c) in Section 3.2 we see that such a $\bar{u}$ can be viewed as product of two configurations modeled on two trees $\mathcal{T}_{1}$ and $\mathcal{T}_{2}$ but one of these configurations contains a vertex of valence three which corresponds to a perturbed curve. At the same time both $\mathcal{P}_{\mathcal{T}_{1}}$ and $\mathcal{P}_{\mathcal{T}_{2}}$ are moduli 
spaces of virtual dimension 0 and so, following the definition of the module action and the pearl differential, they do not contain perturbed curves.

The second case concerns the verification of the associativity type relations involving the module action and it arises if the initial curve leading by bubbling to an element $\bar{u} \in \partial \overline{\mathcal{P}}_{\mathcal{T}}$ is in fact a perturbed curve (satisfying (15)), and carrying 4 marked points, two of which are interior points and each of the two components of the resulting cusp curve carries one interior point. The problem in this case is that just one of the resulting cusp curves satisfies the perturbed equation and the other one is a usual $J$-holomorphic curve (the definition of the marked point selector in this case implies that the lower component in the tree is the perturbed one) and this configuration $\bar{u}$ does not actually appear as an object of type (iii). The reason is that in the relevant moduli spaces all the vertices of valence three correspond to perturbed curves and thus, the configurations of type (iii) in this case contain a cusp curve with both components being perturbed.

The solution to these two issues turns out to be simple: a further analysis of the moduli spaces involved in both cases shows that if the relevant perturbations are small enough - which can be obviously assumed - then the two types of configurations which are compared in each case are in bijection. This is proved by a cobordism argument which is possible because both the perturbed and the unperturbed configurations are regular see again our paper [12] for more details.

\section{Additional tools}

In this section we introduce a number of additional tools which will be useful for the proof of the main theorems and in related computations.

\subsection{Minimal pearls}

As before, we assume here that $L \subset(M, \omega)$ is monotone. Suppose that for some almost complex structure $J$ and Morse function $f: L \rightarrow \mathbb{R}$ the pearl complex $\mathcal{C}^{+}\left(L ; f, \rho_{L}, J\right)$ is defined. It is clear that if $f$ is a perfect Morse function, in the sense that the differential of its Morse complex is trivial, then the pearl complex is most efficient for computations. Clearly, not all manifolds admit perfect Morse functions. However, we will see that, algebraically, we can always reduce the pearl complex to such a minimal form (a similar construction in the cluster set-up has been sketched in [24]).

It is crucial to work here over a "positive" coefficient ring. We will use in this section $\Lambda^{+}=\mathbb{Z}_{2}[t]$. In the algebraic considerations below the fact hat $\operatorname{deg}(t) \leq-2$ plays an important role. 
Let $G$ be a finite dimensional graded $\mathbb{Z}_{2}$-vector space and let $\mathcal{D}=\left(G \otimes \Lambda^{+}, d\right)$ be a chain complex with a differential $d$ which is $\Lambda^{+}$-linear - in other words $\mathcal{D}$ is a $\Lambda^{+}$-chain complex. For an element $x \in G$ let $d(x)=d_{0}(x)+d_{1}(x) t$ with $d_{0}(x) \in G$. In other words $d_{0}$ is obtained from $d(x)$ by treating $t$ as a polynomial variable and putting $t=0$. Clearly $d_{0}: G \rightarrow G, d_{0}^{2}=0$. Similarly, for a chain morphism $\xi$ we denote by $\xi_{0}$ the $d_{0}$-chain morphism obtained by making $t=0$. Let $\mathcal{H}$ be the homology of the complex $\left(G, d_{0}\right)$. We refer to this homology as $d_{0}$-homology in contrast to $d$-homology which is denoted by $H_{*}(\mathcal{D})$.

Proposition 4.1.1 With the notation above there exists a chain complex

$$
\mathcal{D}_{\min }=\left(\mathcal{H} \otimes \Lambda^{+}, \delta\right), \text { with } \delta_{0}=0
$$

and chain maps $\phi: \mathcal{D} \rightarrow \mathcal{D}_{\min }, \psi: \mathcal{D}_{\min } \rightarrow \mathcal{D}$ so that: $\phi \circ \psi=\mathrm{id}, \phi_{0}$ and $\psi_{0}$ induce isomorphisms in $d_{0}$-homology and $\phi$ and $\psi$ induce isomorphisms in $d$-homology. Moreover, the properties above characterize $\mathcal{D}_{\min }$ up to (a generally noncanonical) isomorphism.

Concerning the uniqueness part of the statement see also Section 4.1.1.

Here is an important consequence of Proposition 4.1.1:

Corollary 4.1.2 There exists a complex $\mathcal{C}_{\text {min }}^{+}(L)=\left(H_{*}\left(L ; \mathbb{Z}_{2}\right) \otimes \Lambda^{+}, \delta\right)$, with $\delta_{0}=0$ and so that, for any $(L, f, \rho, J)$ such that $\mathcal{C}^{+}(L ; f, \rho, J)$ is defined, there are chain morphisms $\phi: \mathcal{C}^{+}(L ; f, \rho, J) \rightarrow \mathcal{C}_{\min }^{+}(L)$ and $\psi: \mathcal{C}_{\min }^{+}(L) \rightarrow \mathcal{C}^{+}(L ; f, \rho, J)$ which both induce isomorphisms in quantum homology as well as in Morse homology and satisfy $\phi \circ \psi=\mathrm{id}$. The complex $\mathcal{C}_{\min }^{+}(L)$ with these properties is unique up to (a generally noncanonical) isomorphism.

We call the complex provided by this corollary the minimal pearl complex and the maps $\phi, \psi$ the structure maps associated to $\mathcal{C}^{+}(L ; f, \rho, J)$ (or shorter, to $f$ ). This terminology originates in rational homotopy where a somewhat similar notion is central. There is a slight abuse in this notation as, while any two complexes as provided by the corollary are isomorphic this isomorphism is not canonical. Obviously, in case a perfect Morse function exists on $L$ any pearl complex associated to such a function is already minimal. As mentioned before, in the arguments below it is essential that the differential and morphisms are defined over $\Lambda^{+}$(but the same constructions also work over $\widetilde{\Lambda}^{+}$; see Section 2.1.2 for the various Novikov rings available). In case we need to work over $\Lambda$ we define $\mathcal{C}_{\min }(L)=\mathcal{C}_{\min }^{+}(L) \otimes_{\Lambda^{+}} \Lambda$. 
Remark 4.1.3 (a) An important consequence of the existence of the chain morphisms $\phi$ and $\psi$ is that all the algebraic structures described before (product, module structure etc.) can be transported and computed on the minimal complex. For example, the product is the composition

$$
\begin{aligned}
\mathcal{C}_{\min }^{+}(L) \otimes \mathcal{C}_{\min }^{+}(L) \stackrel{\psi_{1} \otimes \psi_{2}}{\longrightarrow} \mathcal{C}^{+}\left(L ; f_{1}, \rho, J\right) \otimes & \mathcal{C}^{+}\left(L ; f_{2}, \rho, J\right) \\
& \stackrel{*}{\rightarrow} \mathcal{C}^{+}\left(L ; f_{3}, \rho, J\right) \stackrel{\phi_{3}}{\longrightarrow} \mathcal{C}_{\min }^{+}(L)
\end{aligned}
$$

where $\psi_{i}, \phi_{i}$ are the structure maps given by Corollary 4.1.2 and which correspond to the complexes associated to $f_{i}$. There is a cycle in $\mathcal{C}_{\min }^{+}(L)$ equal to $\phi(P)$ where $P$ is the maximum of any Morse function $f$ so that $\mathcal{C}^{+}(L ; f, \rho, J)$ is defined and so that $f$ has a single maximum; $\psi, \phi$ are the associated structure maps. By degree reasons in dimension $n=\operatorname{dim}(L)$ we have $\phi=\phi_{0}$ and $\psi=\psi_{0}$ and so this cycle is independent of the choice of $f$ and of that of the associated structure maps and it coincides with $[L] \otimes 1$ where $[L]$ is the fundamental class of $L$. By a slight abuse of notation we will continue to denote by $[L]$ both the cycle $\phi(P)$ as well as its quantum homology class. In homology, the product defined by (19) has as unity the fundamental class $[L]$. Moreover, with the simplified description of the quantum product given in (13) - where $f_{2}=f_{3}$ we obtain a product so that $[L]$ is the unity at the chain level. It also follows from the fact that $\phi_{0}, \psi_{0}$ induce isomorphisms in Morse homology that the "minimal" product described above is a deformation of the intersection product.

(b) A consequence of point (a) is that $H F(L) \cong Q H(L)=0$ if and only if there is some $x \in \mathcal{C}_{\min }^{+}(L)=H\left(L ; \mathbb{Z}_{2}\right) \otimes \Lambda^{+}$so that $\delta x=[L] t^{k}$. Indeed, suppose that $Q H(L)=0$. Then, as for degree reasons $[L]$ is a cycle in $\mathcal{C}_{\min }(L)$, we obtain that it has to be also a boundary. This means that there exists $a \in \mathcal{C}_{\min }(L)$ so that $\delta a=[L]$. Multiplying $a$ by a large enough positive power $k$ of $t$ gives an element $x=a t^{k}$ which now lies in $\mathcal{C}_{\min }^{+}(L)$ and such that $\delta x=[L] t^{k}$. Conversely, if $\delta x=[L] t^{k}$ then $[L]$ is a boundary in $\mathcal{C}_{\min }(L)$. On the other hand the cycle $[L] \in \mathcal{C}_{\min }(L)$ represents the unity for the product on $H_{*}\left(\mathcal{C}_{\min }(L)\right) \cong Q H_{*}(L)$ just mentioned at point (a) above. Thus the unity is 0 , hence $Q H(L)=0$.

(c) It is also useful to note that there is an isomorphism $Q H(L) \cong H\left(L ; \mathbb{Z}_{2}\right) \otimes \Lambda$ if and only if the differential $\delta$ in $\mathcal{C}_{\min }(L)$ is identically zero.

We now proceed to the proof of the Proposition and of its Corollary.

Proof of Proposition 4.1.1 We start with a useful algebraic property. 
Lemma 4.1.4 Let $\mathcal{D}^{\prime}=\left(G^{\prime} \otimes \Lambda^{+}, d^{\prime}\right)$ and $\mathcal{D}^{\prime \prime}=\left(G^{\prime \prime} \otimes \Lambda^{+}, d^{\prime \prime}\right)$ be two $\Lambda^{+}$-chain complexes. If a chain morphism $\xi: \mathcal{D}^{\prime} \rightarrow \mathcal{D}^{\prime \prime}$ which is $\Lambda^{+}$-linear is so that $\xi_{0}$ induces an isomorphism in $d_{0}$-homology, then $\xi$ induces an isomorphism in $d$-homology.

Proof Recall that the filtration $\mathcal{F}^{k} \Lambda^{+}=t^{k} \Lambda^{+}$induces a filtration, called the degree filtration, on any free $\Lambda^{+}$- module. The resulting spectral sequence induced on any $\Lambda^{+}$-chain complex is called the degree spectral sequence. Clearly, $\xi$ respects the degree filtration and thus it induces a morphism relating the degree spectral sequences of $\mathcal{D}^{\prime}$ and $\mathcal{D}^{\prime \prime}$. We notice that $E^{1}(\xi)$ is identified with the morphism induced by $\xi_{0}$ in $d_{0}$-homology. Therefore, this is an isomorphism. As we work over a field (specifically $\left.\mathbb{Z}_{2}\right)$ this implies that $H_{*}(\xi)$ is an isomorphism.

Remark 4.1.5 (a) Under the assumptions in Lemma 4.1.4, the same spectral sequence argument also shows that the chain morphism

$$
\xi \otimes \operatorname{id}_{\Lambda}: \mathcal{D}^{\prime} \otimes_{\Lambda^{+}} \Lambda \rightarrow \mathcal{D}^{\prime \prime} \otimes_{\Lambda^{+}} \Lambda
$$

induces an isomorphism in homology.

(b) Let $G^{\prime}, G^{\prime \prime}$ be finite dimensional, graded $\mathbb{Z}_{2}$-vector spaces. We claim that a $\Lambda^{+}$-linear morphism

$$
\xi: G^{\prime} \otimes \Lambda^{+} \rightarrow G^{\prime \prime} \otimes \Lambda^{+}
$$

is an isomorphism if and only if $\xi_{0}$ is an isomorphism. Indeed, any such $\xi$ can be viewed as a morphism of chain complexes by assuming that the differentials in the domain and target are trivial. We deduce from Lemma 4.1.4 that, if $\xi_{0}$ is an isomorphism, then $\xi$ is an isomorphism. Conversely, if $\xi$ is an isomorphism, then $t \xi: t\left(G^{\prime} \otimes \Lambda^{+}\right) \rightarrow t\left(G^{\prime \prime} \otimes \Lambda^{+}\right)$is an isomorphism. As $\xi_{0}$ is identified with the quotient morphism

$$
\frac{G^{\prime} \otimes \Lambda^{+}}{t\left(G^{\prime} \otimes \Lambda^{+}\right)} \rightarrow \frac{G^{\prime \prime} \otimes \Lambda^{+}}{t\left(G^{\prime \prime} \otimes \Lambda^{+}\right)}
$$

induced by $\xi$, it follows that $\xi_{0}$ is an isomorphism.

We now return to the proof of Proposition 4.1.1. Start by choosing a basis for the complex $\left(G, d_{0}\right)$ as follows: $G=\mathbb{Z}_{2}\left\langle x_{i}: i \in I\right\rangle \oplus \mathbb{Z}_{2}\left\langle y_{j}: j \in J\right\rangle \oplus \mathbb{Z}_{2}\left\langle y_{j}^{\prime}: j \in J\right\rangle$ so that $d_{0} x_{i}=0, d_{0}\left(y_{j}\right)=0, d_{0} y_{j}^{\prime}=y_{j}, \forall j \in J$. For further use, we denote $B_{X}=\left\{x_{i}: i \in I\right\}, B_{Y}=\left\{y_{j}: j \in J\right\}, B_{Y^{\prime}}=\left\{y_{j}^{\prime}: j \in J\right\}$.

Clearly, $\mathcal{H} \cong \mathbb{Z}_{2}\left\langle x_{i}\right\rangle$ and we will identify further these two vector spaces and denote $\mathcal{D}_{\min }=\mathbb{Z}_{2}\left\langle\tilde{x}_{i}\right\rangle \otimes \Lambda^{+}$where $\tilde{x}_{i}, i \in I$ are of the same degree as the $x_{i}$ 's (the differential on $\mathcal{D}_{\min }$ remains to be defined). We will construct $\phi$ and $\psi$ and $\delta$ so that $\phi_{0}\left(x_{i}\right)=\tilde{x}_{i}$, $\phi_{0}\left(y_{j}\right)=\phi_{0}\left(y_{j}^{\prime}\right)=0, \psi_{0}\left(\tilde{x}_{i}\right)=x_{i}$ and $\delta_{0}=0$. If $\psi_{0}$ and $\phi_{0}$ satisfy these properties, 
then, they induce an isomorphism in $d_{0}$-homology and, by Lemma 4.1.4, $\psi$ and $\phi$ induce isomorphisms in $d$-homology.

The construction is by induction. We fix the following notation: $\mathcal{D}^{k}=\mathbb{Z}_{2}\left\langle x_{i}, y_{j}^{\prime}, y_{j}\right.$ : $\left.\left|x_{i}\right| \geq k,\left|y_{j}^{\prime}\right| \geq k\right\rangle \otimes \Lambda^{+}$. Similarly, we put $\mathcal{D}_{\min }^{k}=\mathbb{Z}_{2}\left\langle\tilde{x}_{i}:\left|x_{i}\right| \geq k\right\rangle \otimes \Lambda^{+}$. Notice that there are some generators in $\mathcal{D}^{k}$ which are of degree $k-1$, namely the $y_{j}$ 's of that degree. With this notation we also see that $\mathcal{D}^{k}$ is a subchain complex of $\mathcal{D}$ (because $d y_{i}^{\prime}=d_{1}\left(y_{i}^{\prime}\right) t$ and so $\left|d_{1}\left(y_{i}^{\prime}\right)\right| \geq\left|y_{i}^{\prime}\right|+1$, the same type of relation holds for $x_{i}$ and for $y_{i}$ we have $\left.d y_{i}=y_{i}^{\prime}+d_{1}\left(y_{i}\right) t\right)$. Assume that $n$ is the maximal degree of the generators in $G$. For the generators of $\mathcal{D}^{n}$ we let $\phi$ be equal to $\phi_{0}$, we put $\delta=0$ on $\mathcal{D}_{\text {min }}^{n}$ and we also let $\psi=\psi_{0}$ on $\mathcal{D}_{\min }^{n}$. To see that $\phi: \mathcal{D}^{n} \rightarrow \mathcal{D}_{\min }^{n}$ is a chain morphism with these definitions it suffices to remark that if $y \in B_{Y},|y|=n-1$, then $y=d_{0} y^{\prime}=d y^{\prime}$ and so $d y=0$.

We now assume $\phi, \delta, \psi$ defined on $\mathcal{D}^{n-s+1}, \mathcal{D}_{\min }^{n-s+1}$ so that $\phi, \psi$ are chain morphisms, they induce isomorphisms in homology and $\phi \circ \psi=\mathrm{id}$. We intend to extend these maps to $\mathcal{D}^{n-s}, \mathcal{D}_{\text {min }}^{n-s}$. We first define $\phi$ on the generators $x \in B_{X}, y^{\prime} \in B_{Y^{\prime}}$ which are of degree $n-s: \phi(x)=\tilde{x}, \phi\left(y^{\prime}\right)=0$. We let $\delta(\tilde{x})=\phi^{n-s+1}(d x)$ (when needed, we use the superscript $(-)^{n-s+1}$ to indicate the maps previously constructed by induction). Here it is important to note that, as $d_{0} x=0$, we have that $d x \in \mathcal{D}^{n-s+1}$. We consider now the generators $y \in B_{Y} \cap \mathcal{D}^{n-s}$ which are of degree $n-s-1$ and we put $\phi(y)=\phi^{n-s+1}\left(y-d y^{\prime}\right)$. This makes sense because $y-d y^{\prime} \in \mathcal{D}^{n-s+1}$. We write $d y^{\prime}=y+y^{\prime \prime}$ and we first see $\phi\left(d y^{\prime}\right)=0=\delta\left(\phi\left(y^{\prime}\right)\right)$ so that, to make sure that $\phi^{n-s}$ is a chain morphism with these definitions, it remains to check that $\delta \phi(y)=\phi(d y)$ for all $y \in B_{Y}$ of degree $n-s-1$. But $\delta \phi(y)=-\delta \phi^{n-s+1}\left(y^{\prime \prime}\right)$ and as $\phi^{n-s+1}$ is a chain morphism, we have $\delta \phi^{n-s+1}\left(y^{\prime \prime}\right)=\phi^{n-s+1} d\left(y^{\prime \prime}\right)$ which implies our identity because $d y^{\prime \prime}+d y=d^{2} y^{\prime}=0$. Clearly, $\phi_{0}^{n-s}$ induces an isomorphism in $d_{0}$-homology and hence in $d$-homology too.

To conclude our induction step it remains to construct the map $\psi$ on the generators $\tilde{x}$ of degree $n-s$. We now consider the difference $d x-\psi^{n-s+1}(\delta \tilde{x})$ and we want to show that there exists $\tau \in \mathcal{D}^{n-s+1}$ so that $d \tau=d x-\psi^{n-s+1}(\delta \tilde{x})$ and $\tau \in$ $\operatorname{ker}\left(\phi^{n-s+1}\right)$. Assuming the existence of this $\tau$ we will put $\psi(\tilde{x})=x-\tau$ and we see that $\psi$ is a chain map and $\phi \circ \psi=$ id. To see that such a $\tau$ exists remark that $w=d x-\psi^{n-s+1}(\delta \tilde{x}) \in \mathcal{D}^{n-s+1}$ and $d w=-d\left(\psi^{n-s+1}(\delta \tilde{x})\right)=-\psi^{n-s+1}(\delta \circ \delta \tilde{x})=0$ (because $\psi^{n-s+1}$ is a chain map). Moreover, $\phi(w)=\phi^{n-s+1}(d x)-\delta \tilde{x}=0$ because $\phi^{n-s+1} \circ \psi^{n-s+1}=\mathrm{id}$. Therefore $w$ is a cycle belonging to $\operatorname{ker}\left(\phi^{n-s+1}\right)$. But $\phi^{n-s+1}$ is a chain morphism which induces an isomorphism in homology and which is surjective. Therefore $H_{*}\left(\operatorname{ker}\left(\phi^{n-s+1}\right)\right)=0$. Thus there exists $\tau \in \operatorname{ker}\left(\phi^{n-s+1}\right)$ so that $d \tau=w$ and this concludes the induction step. 
This construction concludes the first part of the statement and to finish the proof of the proposition we only need to prove the uniqueness result. For this, suppose $\phi^{\prime}: \mathcal{D} \rightarrow \mathcal{D}^{\prime}$ and $\psi^{\prime}: \mathcal{D}^{\prime} \rightarrow \mathcal{D}$ are chain morphisms so that $\phi^{\prime} \circ \psi^{\prime}=$ id with $\mathcal{D}^{\prime}=\left(H \otimes \Lambda^{+}, \delta^{\prime}\right)$, $\delta_{0}^{\prime}=0$ and $H$ some graded, $\mathbb{Z}_{2}$-vector space and $\phi^{\prime}, \psi^{\prime}, \phi_{0}^{\prime}, \psi_{0}^{\prime}$ induce isomorphisms in the respective homologies. We want to show that there exists a chain map $c: \mathcal{D}_{\min } \rightarrow$ $\mathcal{D}^{\prime}$ so that $c$ is an isomorphism. To this end we define $c(u)=\phi^{\prime} \circ \psi(u)$, for all $u \in \mathcal{D}_{\min }$. Now $H_{*}\left(\phi_{0}\right)$ and $H_{*}\left(\phi_{0}^{\prime}\right), H_{*}\left(\psi_{0}\right), H_{*}\left(\psi_{0}^{\prime}\right)$ are all isomorphisms (in $d_{0}$-homology). So $H\left(c_{0}\right)$ is an isomorphism but as $\delta_{0}=0=\delta_{0}^{\prime}$ we deduce that $c_{0}$ is an isomorphism. By Remark 4.1.5 (b), the map $c$ is an isomorphism.

Proof of Corollary 4.1.2 Fix a triple $f^{0}, \rho^{0}, J^{0}$ and assume that $\mathcal{C}^{+}\left(L ; f^{0}, \rho^{0}, J^{0}\right)$ is defined. Apply Proposition 4.1.1 to this complex. Denote by $\left(\mathcal{C}_{\min }^{+}, \phi, \psi\right)$ the resulting minimal complex and the chain morphisms as in the statement of Proposition 4.1.1. The only part of the statement which remains to be proved is that given a different set of data $\left(f^{\prime}, \rho^{\prime}, J^{\prime}\right)$ so that $\mathcal{C}^{+}\left(L ; f^{\prime}, \rho^{\prime}, J^{\prime}\right)$ is defined, there are appropriate morphisms $\phi^{\prime}, \psi^{\prime}$ as in the statement. There are comparison morphisms: $h: \mathcal{C}^{+}\left(L ; f^{\prime}, \rho^{\prime}, J^{\prime}\right) \rightarrow$ $\mathcal{C}^{+}\left(L ; f^{0}, \rho^{0}, J^{0}\right)$ as well as $h^{\prime}: \mathcal{C}^{+}\left(L ; f^{0}, \rho^{0}, J^{0}\right) \rightarrow \mathcal{C}^{+}\left(L ; f^{\prime}, \rho^{\prime}, J^{\prime}\right)$ so that, by construction, both $h$ and $h^{\prime}$ are inverse in homology and both induce isomorphisms in Morse homology (and these two isomorphisms are also inverse). Define $\phi^{\prime}: \mathcal{C}^{+}\left(L ; f^{\prime}, \rho^{\prime}, J^{\prime}\right) \rightarrow \mathcal{C}_{\min }^{+}, \psi^{\prime \prime}: \mathcal{C}_{\min }^{+} \rightarrow \mathcal{C}^{+}\left(L ; f^{\prime}, \rho^{\prime}, J^{\prime}\right)$ by $\phi^{\prime}=\phi \circ h$ and $\psi^{\prime \prime}=h^{\prime} \circ \psi$. It is clear that $\phi^{\prime}, \psi^{\prime \prime}, \phi_{0}^{\prime}$ and $\psi_{0}^{\prime \prime}$ induce isomorphisms in homology. Moreover, as $h_{0}$ and $h_{0}^{\prime}$ are inverse in homology and $\delta_{0}=0$ in $\mathcal{C}_{\min }^{+}$it follows that $\phi_{0}^{\prime} \circ \psi_{0}^{\prime \prime}=\mathrm{id}$. This means by Lemma 4.1.4 that $v=\phi^{\prime} \circ \psi^{\prime \prime}$ is a chain isomorphism so that $v_{0}$ is the identity. We now put $\psi^{\prime}=\psi^{\prime \prime} \circ v^{-1}$ and this satisfies all the needed properties. The uniqueness of $\mathcal{C}_{\min }^{+}(L)$ now follows from the uniqueness part in Proposition 4.1.1.

4.1.1 Further remarks on minimal models While the minimal complex $\mathcal{D}_{\text {min }}$ associated to $\Lambda^{+}$-complex $\mathcal{D}$ is unique (up to isomorphism), this is not the case for the structural maps $\phi$ and $\psi$. For these maps we expect uniqueness in a weaker sense such as uniqueness up to chain homotopy, however we will not pursue this direction here. On the other hand, we will use minimal models in Section 5 quite frequently. In fact, in Section 5 we will have to use the specific choice of the morphisms $\phi, \psi$ (as well as $\left.\phi_{0}, \psi_{0}\right)$ that is constructed in the proof of Proposition 4.1.1. It seems plausible that this can be avoided by axiomatizing more the theory of minimal models, but we will not do this here since we view the minimal model as a purely computational tool. 


\subsection{Geometric criterion for the vanishing of $Q H(L)$}

Let $L \subset(M, \omega)$ be a monotone Lagrangian submanifold. Remark 4.1.3 (b) provides a criterion for the vanishing of $Q H(L)$. We provide here a more geometric such criterion which is useful when $N_{L}=2$ which we will assume in this section.

Let $\partial: H_{2}(M, L ; \mathbb{Z}) \rightarrow H_{1}(L ; \mathbb{Z})$ denote the boundary homomorphism and denote by $\partial_{\mathbb{Z}_{2}}: H_{2}(M, L ; \mathbb{Z}) \rightarrow H_{1}\left(L ; \mathbb{Z}_{2}\right)$ the composition of $\partial$ with the reduction mod 2 , $H_{1}(L ; \mathbb{Z}) \rightarrow H_{1}\left(L ; \mathbb{Z}_{2}\right)$. Given $A \in H_{2}^{D}(M, L)$ and $J \in \mathcal{J}(M, \omega)$ consider the evaluation map

$$
\mathrm{ev}_{A, J}:(\mathcal{M}(A, J) \times \partial D) / G \longrightarrow L, \quad \operatorname{ev}_{A, J}(u, p)=u(p),
$$

where $G=\operatorname{Aut}(D) \cong \operatorname{PSL}(2, \mathbb{R})$ is the group of biholomorphisms of the disk.

For every $J \in \mathcal{J}(M, \omega)$ let $\mathcal{E}_{2}(J)$ be the set of all classes $A \in H_{2}^{D}(M, L)$ with $\mu(A)=2$ for which there exist $J$-holomorphic disks with boundary on $L$ in the class $A$ :

$$
\mathcal{E}_{2}(J)=\left\{A \in H_{2}^{D}(M, L) \mid \mu(A)=2, \mathcal{M}(A, J) \neq \varnothing\right\} .
$$

Define:

$$
\mathcal{E}_{2}=\bigcap_{J \in \mathcal{J}(M, \omega)} \mathcal{E}_{2}(J)
$$

Standard arguments show that:

(1) $\mathcal{E}_{2}(J)$ is a finite set for every $J$.

(2) There exists a second category subset $\mathcal{J}_{\text {reg }} \subset \mathcal{J}(M, \omega)$ such that for every $J \in \mathcal{J}_{\text {reg }}, \mathcal{E}_{2}(J)=\mathcal{E}_{2}$. In other words, for generic $J, \mathcal{E}_{2}(J)$ is independent of $J$.

(3) For every $J \in \mathcal{J}$ and every $A \in \mathcal{E}_{2}(J)$ the space $\mathcal{M}(A, J)$ is compact and all disks $u \in \mathcal{M}(A, J)$ are simple.

(4) For $J \in \mathcal{J}_{\text {reg }}$ and $A \in \mathcal{E}_{2}$, the space $(\mathcal{M}(A, J) \times \partial D) / G$ is a compact smooth manifold without boundary. Its dimension is $n=\operatorname{dim} L$. In particular, for generic $x \in L$, the number of $J$-holomorphic disks $u \in \mathcal{M}(A, J)$ with $u(\partial D) \ni x$ is finite.

(5) For every $A \in \mathcal{E}_{2}$ and $J_{0}, J_{1} \in \mathcal{J}_{\text {reg }}$ the manifolds $\left(\mathcal{M}\left(A, J_{0}\right) \times \partial D\right) / G$ and $\left(\mathcal{M}\left(A, J_{1}\right) \times \partial D\right) / G$ are cobordant via a compact cobordism. Moreover, the evaluation maps ev $\mathrm{ev}_{A, J_{0}}, \mathrm{ev}_{A, J_{1}}$ extend to this cobordism, hence $\operatorname{deg}_{\mathbb{Z}_{2}} \operatorname{ev}_{A, J_{0}}=$ $\operatorname{deg}_{\mathbb{Z}_{2}} \operatorname{ev}{ }_{A, J_{1}}$. In other words $\operatorname{deg}_{\mathbb{Z}_{2}} \operatorname{ev}_{A, J}$ depends only on $A \in \mathcal{E}_{2}$.

(6) In fact, the set $\mathcal{J}_{\text {reg }}$ above can be taken to be the set of all $J \in \mathcal{J}(M, \omega)$ which are regular for all classes $A \in H_{2}^{D}(M, L)$ in the sense that the linearization of the $\bar{\partial}_{J}$ operator is surjective at every $u \in \mathcal{M}(A, J)$. 
Let $J \in \mathcal{J}_{\text {reg }}$ and let $x \in L$ be a generic point. Define a one dimensional $\mathbb{Z}_{2}$-cycle $\delta_{x}(J)$ to be the sum of the boundaries of all $J$-holomorphic disks with $\mu=2$ whose boundaries pass through $x$. Of course, if a disk meets $x$ along its boundary several times we take its boundary in the sum with appropriate multiplicity. Thus the precise definition is

$$
\delta_{x}(J)=\sum_{A \in \mathcal{E}_{2}} \sum_{(u, p) \in \mathrm{ev}_{A, J}^{-1}(x)} u(\partial D) .
$$

By the preceding discussion the homology class $D_{1}=\left[\delta_{x}(J)\right] \in H_{1}\left(L ; \mathbb{Z}_{2}\right)$ is independent of $J$ and $x$. In fact

$$
D_{1}=\sum_{A \in \mathcal{E}_{2}}\left(\operatorname{deg}_{\mathbb{Z}_{2}} \operatorname{ev}_{A, J}\right) \partial_{\mathbb{Z}_{2}} A
$$

Proposition 4.2.1 Let $L \subset(M, \omega)$ be a monotone Lagrangian submanifold with $N_{L}=2$. If $D_{1} \neq 0$ then $Q H_{*}(L)=0$.

Proof Choose a generic $J \in \mathcal{J}(M, \omega)$. Let $f: L \rightarrow \mathbb{R}$ be a generic Morse function with precisely one local maximum at a point $x \in L$ and fix a generic Riemannian metric on $L$. Denote by $\left(C M_{*}(f), \partial_{0}\right),\left(\mathcal{C}_{*}(f, J), d\right)$ the Morse and pearl complexes associated to $f, J$ and the chosen Riemannian metric. As discussed in Section 3.2 (b), $x$ is a cycle in the pearl complex of $f$ and its quantum homology class is the unity.

For degree reasons the restriction of $d$ to $C M_{n-1}(f) \subset \mathcal{C}_{n-1}(f, J)$ is given by $d=\partial_{0}+\partial_{1} t$, where $\partial_{1}: C M_{n-1}(f) \rightarrow C M_{n}(f)=\mathbb{Z}_{2} x$ counts pearly trajectories with holomorphic disks of Maslov index 2. Since $x$ is a maximum of $f$, no $-\nabla f$ trajectories can enter $x$ (ie $W_{x}^{s}(f)=\{x\}$ ). Therefore for every $y \in \operatorname{Crit}_{n-1}(f)$ we have

$$
\partial_{1} y=\#_{\mathbb{Z}_{2}}\left(W_{y}^{u}(f) \cap \delta_{x}(J)\right) x .
$$

Assume now that $D_{1} \neq 0$. By Poincaré duality there exists an $(n-1)$-dimensional cycle $C$ in $L$ such that

$$
\#_{\mathbb{Z}_{2}} C \cap \delta_{x}(J) \neq 0 \text {. }
$$

Let $z \in C M_{n-1}(f)$ be a $\partial_{0}$-cycle representing $[C] \in H_{n-1}\left(L ; \mathbb{Z}_{2}\right)$. Then

$$
d(z)=\partial_{1}(z) t=\#_{\mathbb{Z}_{2}}\left(W_{z}^{u}(f) \cap \delta_{x}(J)\right) x t=\#_{\mathbb{Z}_{2}}\left(C \cap \delta_{x}(J)\right) x t=a x t
$$

for some nonzero scalar $a$. (Of course, $a \neq 0$ is the same as $a=1$ here, since we work over $\mathbb{Z}_{2}$. However we wrote $a x$ to emphasize that the argument works over every field.) It follows that $[x]=0 \in Q H_{n}(L)$. But, as $[x]$ is the unity of $Q H_{*}(L)$, we deduce $Q H_{*}(L)=0$. 


\subsection{Action of the symplectomorphism group}

We now describe a property of our machinery which is very useful in computations when symmetry is present. In this section $\mathcal{R}$ is any of the rings described in Section 2.1.2.

Proposition 4.3.1 Let $\phi: L \rightarrow L$ be a diffeomorphism which is the restriction to $L$ of an ambient symplectic diffeomorphism $\bar{\phi}$ of $M$. Let $f, \rho, J$ be so that the pearl complex $\mathcal{C}(L ; \mathcal{R} ; f, \rho, J)$ is defined. There exists a chain map

$$
\tilde{\phi}: \mathcal{C}(L ; \mathcal{R} ; f, \rho, J) \rightarrow \mathcal{C}(L ; \mathcal{R} ; f, \rho, J)
$$

which respects the degree filtration, induces an isomorphism in homology, and so that the morphism $E^{1}(\widetilde{\phi})$ induced by $\widetilde{\phi}$ at the $E^{1}$ level of the degree spectral sequence coincides with $H_{*}(\phi) \otimes \mathrm{id}_{\Lambda^{+}}$(where $H_{*}(\phi)$ is the isomorphism induced by $\phi$ on singular homology). The map $\bar{\phi} \rightarrow \widetilde{\phi}$ induces a representation

$$
\bar{h}: \operatorname{Symp}(M, L) \rightarrow \operatorname{Aut}\left(Q H_{*}(L ; \mathcal{R})\right)
$$

where $\operatorname{Aut}\left(Q H_{*}(L ; \mathcal{R})\right)$ are the ring automorphisms of $Q H_{*}(L ; \mathcal{R})$ preserving the augmentation and $\operatorname{Symp}(M, L)$ are the symplectomorphisms of $M$ which keep $L$ invariant. The restriction of $\bar{h}$ to $\operatorname{Symp}_{0}(M) \cap \operatorname{Symp}(M, L)$ takes values in the automorphisms of $Q H(L ; \mathcal{R})$ as an algebra over $Q H(M ; \mathcal{R})$ (here $\operatorname{Symp}_{0}(M)$ is the component of the identity in $\operatorname{Symp}(M))$.

Proof To ease notation, we omit the ring $\mathcal{R}$ in the writing of the pearl complexes below.

Assume that $\phi: L \rightarrow L$ is a diffeomorphism which is the restriction to $L$ of the symplectomorphism $\bar{\phi}$ and $f, \rho, J$ are such that the chain complex $\mathcal{C}(L ; f, \rho, J)$ is defined. Let $f^{\phi}=f \circ \phi^{-1}$. There exists a basis preserving isomorphism

$$
h^{\phi}: \mathcal{C}(L ; f, \rho, J) \rightarrow \mathcal{C}\left(L ; f^{\phi}, \rho^{*}, J^{*}\right)
$$

induced by $x \rightarrow \phi(x)$ for all $x \in \operatorname{Crit}(f)$ where $\rho^{*}, J^{*}$ are obtained by the pushforward of $\rho, J$ by means of $\phi$ and the symplectomorphism $\bar{\phi}$. The isomorphism $h^{\phi}$ acts in fact as an identification of the two complexes.

Next, there is also the standard comparison chain morphism, canonical up to chain homotopy

$$
c: \mathcal{C}\left(L ; f^{\phi}, \rho^{*}, J^{*}\right) \rightarrow \mathcal{C}(L ; f, \rho, J) .
$$

We now consider the composition $\tilde{\phi}=c \circ h^{\phi}$. It is clear that this map induces an isomorphism in homology and that it preserves the ring structure and the augmentation 
(as each of its factors does so). We now inspect the Morse theoretic analogue of these morphisms - in the sense that we consider instead of the complexes $\mathcal{C}(L ; f,-,-)$ the respective Morse complexes $C(f,-)$. It is easy to see that the Morse theoretic version of $\widetilde{\phi}$ induces in Morse homology precisely $H_{*}(\phi)$. But this means that at the $E^{1}$ stage of the degree filtration the morphism induced by $\tilde{\phi}$ has the form $H_{*}(\phi) \otimes \mathrm{id}_{\mathcal{R}}$.

We now denote $k=\bar{h}(\bar{\phi})$ and we need to verify that for any two elements $\bar{\phi}, \bar{\psi} \in$ $\operatorname{Symp}(M, L)$ we have $\bar{h}(\bar{\phi} \circ \bar{\psi})=\bar{h}(\phi) \circ \bar{h}(\psi)$. It is easy to see that this is implied by the commutativity of the diagram

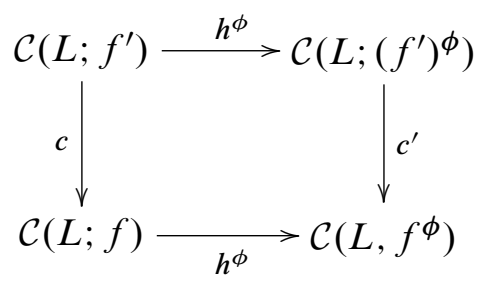

for any two Morse function $f$ and $f^{\prime}$ so that the respective complexes are defined. To verify this commutativity, first we use some homotopy $H$, joining $f$ to $f^{\prime}$, to provide the comparison morphism $c$ and we then use the homotopy $H \circ \phi^{-1}$ to define $c^{\prime}$.

Finally, recall that the module structure of $Q H(L)$ over $Q H(M)$ is defined by using an additional Morse function $F: M \rightarrow \mathbb{R}$. If we put $F^{\bar{\phi}}=F \circ \bar{\phi}^{-1}$ we see easily that the external operations defined by using $f, F, \rho, J$ and $f^{\phi}, F^{\widehat{\phi}}, \rho^{*}, J^{*}$ are identified one to the other via the application $h^{\phi}$ (extended in the obvious way to the critical points of $F$ ). There is a usual comparison map $\bar{c}$ relating the Morse complex of $F^{\bar{\phi}}$ to that of $F$. Together with $c$ the map $\bar{c}$ identifies - in homology - the external product associated to $f^{\phi}, F^{\bar{\phi}}, \rho^{*}, J^{*}$ and the external product associated to $f, F, \rho, J$. At the level of the quantum homology of $M$ the composition $\bar{c} \circ h^{\phi}$ induces $H_{*}(\bar{\phi}) \otimes \mathrm{id}_{\mathcal{R}}$. Therefore, if $\bar{\phi} \in \operatorname{Symp}_{0}(M)$, it follows that this last map is the identity and proves the claim.

Remark 4.3.2 It results from the proof above that for $\bar{h}(\phi)$ to be an algebra automorphism it is sufficient that $\bar{\phi}$ induce the identity at the level of the singular homology of $M$, eg when $\phi$ is homotopic to the identity.

\subsection{Duality}

We start by fixing some algebraic notation and conventions. Let $\mathcal{R}$ be a commutative $\tilde{\Lambda}^{+}$algebra. Suppose that $(\mathcal{C}, \partial)$ is a free $\mathcal{R}$-chain complex. Thus $\mathcal{C}=G \otimes \mathcal{R}$ with $G$ some graded $\mathbb{Z}_{2}$-vector space. We let

$$
\mathcal{C}^{\odot}=\operatorname{hom}_{\mathcal{R}}(\mathcal{C}, \mathcal{R})
$$


graded so that the degree of a morphism $g: \mathcal{C} \rightarrow \mathcal{R}$ is $k$ if $g$ takes $\mathcal{C}_{l}$ to $\mathcal{R}_{l+k}$ for all $l$.

Let $\mathcal{C}^{\prime}=\operatorname{hom}_{\mathbb{Z}_{2}}\left(G, \mathbb{Z}_{2}\right) \otimes \mathcal{R}$ be graded such that if $x$ is a basis element of $G$, then its dual $x^{*} \in \mathcal{C}^{\prime}$ has degree $\left|x^{*}\right|=-|x|$. There is an obvious degree preserving isomorphism $\psi: \mathcal{C}^{\odot} \rightarrow \mathcal{C}^{\prime}$ defined by $\psi(f)=\sum_{i} f\left(g_{i}\right) g_{i}^{*}$ where $\left(g_{i}\right)$ is a basis of $G$ and $\left(g_{i}^{*}\right)$ is the dual basis. We define the differential of $\mathcal{C}^{\odot}, \partial^{*}$, as the adjoint of $\partial$ :

$$
\left\langle\partial^{*} f, x\right\rangle=\langle f, \partial x\rangle, \forall x \in \mathcal{C}, f \in \mathcal{C}^{\odot} .
$$

Clearly, $\mathcal{C}^{\odot}$ continues to be a chain complex (and not a co-chain complex).

An additional algebraic notion will be useful: the co-chain complex $\mathcal{C}^{*}$ associated to $\mathcal{C}$. To define it, for a graded $\mathbb{Z}_{2}$-vector space $V$ let $V^{\text {inv }}$ be the graded vector space obtained by reversing the degree of the elements in $V:$ if $v \in V^{\text {inv }}$, then its degree is $|v|=-\operatorname{deg}_{V}(v)$. Clearly, $(V \otimes W)^{\text {inv }}=V^{\text {inv }} \otimes W^{\text {inv }}$.

For the complex $\mathcal{C}$ as above we let $\mathcal{C}^{*}=\left(\mathcal{C}^{\odot}\right)^{\text {inv }}=\operatorname{hom}_{\mathbb{Z}_{2}}\left(G, \mathbb{Z}_{2}\right)^{\text {inv }} \otimes \mathcal{R}^{\text {inv }}$. The complex $\mathcal{C}^{*}$ is obviously a co-chain complex and its differential is a $\mathcal{R}^{\text {inv }}$-module map. The cohomology of $\mathcal{C}$ is then defined as $H^{k}(\mathcal{C})=H^{k}\left(\mathcal{C}^{*}\right)$. Obviously, there is a canonical isomorphism: $H_{-k}\left(\mathcal{C}^{\odot}\right) \cong H^{k}\left(\mathcal{C}^{*}\right)$.

A particular case of interest here is when $\mathcal{C}=\mathcal{C}(L ; \mathcal{R} ; f, \rho, J)$. In this case we denote

$$
Q H^{k}(L ; \mathcal{R})=H^{k}\left(\mathcal{C}(L ; \mathcal{R} ; f, \rho, J)^{*}\right) .
$$

Notice that the chain morphisms $\eta: \mathcal{C} \rightarrow \mathcal{C}^{\odot}$ of degree $-n$ are in 1-1 correspondence with the chain morphisms of degree $-n$ :

$$
\tilde{\eta}: \mathcal{C} \otimes_{\mathcal{R}} \mathcal{C} \rightarrow \mathcal{R}
$$

via the formula $\tilde{\eta}(x \otimes y)=\eta(x)(y)$. Here the ring $\mathcal{R}$ on the right hand-side is considered as a chain complex with trivial differential.

For $n \in \mathbb{Z}$ and any chain complex $\mathcal{C}$ as before we let $s^{n} \mathcal{C}$ be its $n$-fold suspension. This is a chain complex which coincides with $\mathcal{C}$ but it is graded so that the degree of $x$ in $s^{n} \mathcal{C}$ is $n+$ the degree of $x$ in $\mathcal{C}$. A particular useful case where both duality and suspension appear is in the following sequence of obvious isomorphisms: $H_{k}\left(s^{n} \mathcal{C}^{\odot}\right) \cong$ $H_{k-n}\left(\mathcal{C}^{\odot}\right) \cong H^{n-k}\left(\mathcal{C}^{*}\right)$.

Proposition 4.4.1 Let $n=\operatorname{dim}(L)$. There exists a degree preserving morphism of chain complexes

$$
\eta: \mathcal{C}(L ; \mathcal{R} ; f, \rho, J) \rightarrow s^{n}\left(\mathcal{C}(L ; \mathcal{R} ; f, \rho, J)^{\odot}\right)
$$


which is a morphism of $\mathcal{R}$-modules and induces an isomorphism in homology. In particular, we have an isomorphism: $\eta: Q H_{k}(L ; \mathcal{R}) \rightarrow Q H^{n-k}(L ; \mathcal{R})$. The corresponding $($ degree $-n)$ bilinear map

$$
H\left(\tilde{\eta}: Q H(L ; \mathcal{R}) \otimes_{\mathcal{R}} Q H(L ; \mathcal{R}) \rightarrow \mathcal{R}\right.
$$

coincides with the product described at point (ii) of Theorem A composed with the augmentation $\epsilon_{L}$. When $\mathcal{R}=\Lambda$ the pairing $H(\widetilde{\eta})$ is nondegenerate. Moreover, for any $k \in \mathbb{Z}$ the induced pairing

$$
H(\widetilde{\eta})^{0}: Q H_{k}(L) \otimes_{\mathbb{Z}_{2}} Q H_{n-k}(L) \rightarrow \Lambda_{0}=\mathbb{Z}_{2}
$$

is nondegenerate.

Proof of Proposition 4.4.1 For any two pearl complexes $\mathcal{C}\left(L ; \mathcal{R} ; f, \rho_{L}, J\right)$ and $\mathcal{C}\left(L ; \mathcal{R} ; f^{\prime}, \rho_{L}^{\prime}, J^{\prime}\right)$ the construction at point Section 3.2 (e). provides a comparison chain morphism relating them. There is an alternative way to construct a comparison map

$$
\phi^{f, f^{\prime}}: \mathcal{C}\left(L ; \mathcal{R} ; f, \rho_{L}, J\right) \rightarrow \mathcal{C}\left(L ; \mathcal{R} ; f^{\prime}, \rho_{L}, J^{\prime}\right)
$$

in case $f$ and $f^{\prime}$ are in general position (and, to simplify the argument below, we use the same Riemannian metric $\rho_{L}$ for both $f$ and $f^{\prime}$ ). In homology, this induces the same morphism as the one provided by the map $\phi^{F, \widetilde{\rho}_{L}, \widetilde{J}}$ constructed at point (e) in Section 3.2. This alternative comparison map is useful in the understanding of duality. The definition of this map is

$$
\phi^{f, f^{\prime}}(x)=\sum_{\mathcal{T}^{\prime}} \#\left(\mathcal{P}_{\mathcal{T}^{\prime}}\right) y t^{\mu\left[\mathcal{T}^{\prime}\right] / N_{L}}
$$

where the sum is taken over all the trees $\mathcal{T}^{\prime}$ of symbol $(x: y), x \in \operatorname{Crit}(f), y \in \operatorname{Crit}\left(f^{\prime}\right)$ and $|x|-|y|+\mu\left(\left[\mathcal{T}^{\prime}\right]\right)=0$. We put in this case $f_{1}=f$ and $f_{2}=f^{\prime}$. The exit rule - point (v) in Section 3.1 A - needs to be slightly modified for these trees: in the tree $\mathcal{T}^{\prime}$ there is one special vertex $v_{0}$ so that for all vertices above it the exit rule is $\Theta\left(f_{1}\right)=f_{1}$, for all the vertices below it the exit rule is $\Theta\left(f_{2}\right)=f_{2}$ and at $v_{0}$ the exit rule is $\Theta\left(f_{1}\right)=f_{2}$. Condition (iv) in Section 3.1 A is also slightly modified in the sense that the vertex $v_{0}$ is allowed to satisfy $\omega\left(\left[v_{0}\right]\right)=0$. The marked point selector is as at point Section 3.2 (a). The duality map

$$
\eta: \mathcal{C}(L ; \mathcal{R} ; f, \rho, J) \rightarrow s^{n}\left(\mathcal{C}(L ; \mathcal{R} ; f, \rho, J)^{\odot}\right)
$$

is defined as the composition $\eta=\eta_{f}^{\prime} \circ \phi^{F, \rho_{L}, J}$ where the map $\eta_{f}^{\prime}$ is the canonical identification of chain complexes obtained by "reversing" the flow $\eta_{f}^{\prime}: \mathcal{C}(L ; \mathcal{R} ;-f, \rho, J) \rightarrow$ $s^{n}\left(\mathcal{C}(L ; \mathcal{R} ; f, \rho, J)^{\odot}\right)$ (sending each critical point $x \in \mathrm{Crit}_{k}(-f)$ to $x \in \mathrm{Crit}_{n-k}(f)$ ) and the map $\phi^{F, \rho_{L}, J}$ is the comparison map associated to a Morse homotopy $F$ 
between $f$ and $-f$. To prove the identity $H(\widetilde{\eta})=\epsilon_{L}(-*-)$, let $f^{\prime}$ be another Morse function in generic position with $f$. In homology $\eta_{*}=\phi_{*}^{G, \rho_{L}, J} \circ\left(\eta_{f^{\prime}}^{\prime}\right)_{*} \circ \phi_{*}^{F^{\prime}, \rho_{L}, J}$ where $F^{\prime}$ is a Morse homotopy from $f$ to $-f^{\prime}$ and $G$ is a Morse homotopy from $f^{\prime}$ to $f$. Thus we also have $\eta_{*}=\phi_{*}^{G, \rho_{L}, J} \circ\left(\eta_{f^{\prime}}^{\prime}\right)_{*} \circ \phi_{*}^{f,-f^{\prime}}$. The relation we want to justify follows by comparing the moduli spaces associated to the trees $\mathcal{T}^{\prime}$ of symbol $(x: y)$ with $x \in \operatorname{Crit}(f), y \in \operatorname{Crit}\left(-f^{\prime}\right)$ used in the definition of $\phi^{f,-f^{\prime}}$ and the moduli spaces associated to trees $\mathcal{T}$ of symbol $(x, y: m)$ (with $f=f_{1}, f^{\prime}=f_{2}$ ) where $x \in \operatorname{Crit}(f), y \in \operatorname{Crit}\left(f^{\prime}\right), m \in \operatorname{Crit}_{0}\left(f_{3}\right)$ used in the definition of the product $-*-$ at the point (b) in Section 3.2. Here $m$ is the unique minimum of the function $f_{3}$. Indeed it is immediate to see that the 0 -dimensional such moduli spaces are in bijection and this implies the claimed identity.

It remains to prove that the pairing $H(\widetilde{\eta})^{0}$ (and thus $H(\widetilde{\eta})$ ) is nondegenerate when $\mathcal{R}=\Lambda$. From now on we put $\mathcal{R}=\Lambda$ and omit it from the notation.

Let $\mathcal{C}$ be a finite rank free $\Lambda$-chain complex $(\operatorname{eg} \mathcal{C}=\mathcal{C}(L ; f, \rho, J))$. Consider the following pairing:

$\Theta: H_{k}(\mathcal{C}) \otimes H_{-k}\left(\mathcal{C}^{\odot}\right) \rightarrow \Lambda_{0}=\mathbb{Z}_{2}$,

which is defined as follows. Given two classes $a \in H_{k}(\mathcal{C}), g \in H_{-k}\left(\mathcal{C}^{\odot}\right)$ choose cycles representing them, $a=[\alpha], g=[\varphi]$, and define $\Theta(a \otimes g)=\varphi(\alpha)$. It is easy to see that $\Theta$ is well defined. We will prove below the following.

\section{Lemma 4.4.2 The pairing $\Theta$ is nondegenerate.}

Note that in view of the canonical isomorphisms $Q H_{*}(L) \cong H_{*-n}\left(\mathcal{C}(L ; f, \rho, J)^{\odot}\right)$ the nondegeneracy of $\Theta$ (for $\mathcal{C}=\mathcal{C}(L ; f, \rho, J)$ ) implies that $H(\widetilde{\eta})^{0}$ is nondegenerate.

We now proceed to prove Lemma 4.4.2. Given $l \in \mathbb{Z}$ denote by $\left(\operatorname{hom}_{\Lambda}(H(\mathcal{C}), \Lambda)\right)_{l}$ the space of $\Lambda$-linear morphisms $h: H(\mathcal{C}) \rightarrow \Lambda$ that have degree $l$. Consider now the following canonical map:

$$
\rho: H_{l}\left(\mathcal{C}^{\odot}\right) \rightarrow\left(\operatorname{hom}_{\Lambda}(H(\mathcal{C}), \Lambda)\right)_{l},
$$

defined as follows. Given $g \in H_{l}\left(\mathcal{C}^{\odot}\right)$, choose a cycle $\varphi \in \mathcal{C}_{l}^{\odot}=\left(\operatorname{hom}_{\Lambda}(\mathcal{C}, \Lambda)\right)_{l}$ that represents $g$. Clearly, $\varphi$ descends to a map $H_{*}(\mathcal{C}) \rightarrow \Lambda_{*+l}$ which we define to be $\rho(g)$. It is easy to see that the map $\rho$ is well defined. Note also that we have

$$
\Theta(a \otimes g)=\rho(g)(a), \quad \forall a \in H_{k}(\mathcal{C}), g \in H_{-k}\left(\mathcal{C}^{\odot}\right) .
$$

Lemma 4.4.3 Let $\mathcal{C}$ be as above. Then for every $l \in \mathbb{Z}$ the map $\rho$ is an isomorphism. 
Before proving this lemma let us see how it implies the nondegeneracy of $\Theta$ (hence that of $\left.H(\widetilde{\eta})^{0}\right)$.

Proof that $\Theta$ is nondegenerate Let $0 \neq a \in H_{k}(\mathcal{C})$. Choose a homomorphism $\phi_{k}: H_{k}(\mathcal{C}) \rightarrow \Lambda_{0}=\mathbb{Z}_{2}$ with $\phi_{k}(a) \neq 0$. Extend $\phi_{k}$ to a $\Lambda$-linear homomorphism $\phi: H_{*}(\mathcal{C}) \rightarrow \Lambda_{*-k}$ (this extension can be done by linearity over $\Lambda$ in degrees $*=$ $k+q N_{L}$ and by 0 in all other degrees). Clearly $\Theta\left(a \otimes \rho^{-1}(\phi)\right)=\phi(a) \neq 0$.

Assume now that $0 \neq g \in H_{-k}\left(\mathcal{C}^{\odot}\right)$. Then $\rho(g): H_{*}(\mathcal{C}) \rightarrow \Lambda_{*-k}$ is a nontrivial homomorphism. This means that there exists $j \in \mathbb{Z}$ and $b \in H_{j}(\mathcal{C})$ such that $\rho(g)(b) \neq$ 0 . As $\rho(g)(b) \in \Lambda_{j-k}$ it follows that $N_{L} \mid(j-k)$. Put $a=t^{(j-k) / N_{L}} b \in H_{k}(\mathcal{C})$. Clearly $\rho(g)(a) \neq 0$, which implies that $\Theta(a \otimes g) \neq 0$. This concludes the proof of the nondegeneracy of $\Theta$, modulo the proof of Lemma 4.4.3.

To prove Lemma 4.4.3 we need some more preparation. Let $\mathcal{R}$ be a commutative graded ring and $M$ a graded $\mathcal{R}$-module. Denote by $\pi_{i}: M \rightarrow M_{i}$ the projection on the $i$-th component of $M$. Let $N \subset M$ be a submodule. We say that $N$ is a graded submodule if for every $x \in N$ we have $\pi_{i}(x) \in N$ for every $i \in \mathbb{Z}$. In that case the grading of $M$ induces a grading on $N$ and $N$ becomes a graded $\mathcal{R}$-module by itself. Note that not every submodule of a graded module is graded. However:

Lemma 4.4.4 (i) A submodule $N \subset M$ is a graded submodule if and only if it is generated (over $\mathcal{R}$ ) by a collection $\left\{x_{s}\right\}_{s \in \mathcal{S}}$ of homogeneous elements. In particular, if $N_{1}, N_{2} \subset M$ are graded submodules then so is $N_{1}+N_{2}$.

(ii) Let $\mathcal{R}=\Lambda$. Let $M$ be a free finite rank graded $\Lambda$-module and $N \subset M$ a graded submodule. Then there exists a graded submodule $Q \subset M$ which is a complement of $N$, ie $N \oplus Q=M$.

Proof The proof of statement (i) is straightforward, so we omit it.

We prove (ii). Choose a homogeneous element $x_{1} \in M \backslash N$ (if there are no such elements clearly $N=M$ ). Put $Q^{(1)}=\Lambda x_{1}$. We claim that $N \cap Q^{(1)}=0$. Indeed, assume that $0 \neq \lambda x_{1} \in N$ for some $\lambda \in \Lambda$. As $x_{1}$ is homogeneous and $N$ is a graded submodule, all the homogeneous components of $\lambda x_{1}$ must lie in $N$. In particular there exists $r \in \mathbb{Z}$ such that $t^{r} x_{1} \in N$. As $t^{r}$ is invertible it follows that $x_{1} \in N$. A contradiction.

We now continue the same construction inductively, namely we choose a homogeneous element $x_{2} \in M \backslash\left(N+Q^{(1)}\right)$. We claim that $\Lambda x_{2} \cap\left(N+Q^{(1)}\right)=0$. The argument is similar to the preceding one (for $N \cap \Lambda x_{1}=0$ ). The point is that $N+Q^{(1)}$ is a 
graded submodule. Put $Q^{(2)}=Q^{(1)}+\Lambda x_{2}$. Clearly we have $N \cap Q^{(2)}=0$. Note also that $Q^{(2)}$ and $N+Q^{(2)}$ are both graded submodules of $M$.

Continuing this inductive construction we obtain, after a finite number of steps $v$, the desired complement $Q=Q^{(v)}$ which satisfies $N \oplus Q=M$. It is important here that $M$ is free of finite rank and that $\Lambda$ is a PID. These two conditions assure that every submodule of $M$ is also free with rank $\leq$ the rank of $M$. In particular the process of defining $Q$ concludes in a finite number of steps.

Remark 4.4.5 We remark that the statement at point (ii) does not seem to hold if we replace $\Lambda$ by more general graded rings $\mathcal{R}$. In order for the proof above to work we need that every nontrivial element in each $\mathcal{R}_{j}(\forall j \in \mathbb{Z})$ is invertible. This obviously holds for $\mathcal{R}=\Lambda$, but not for $\mathcal{R}=\Lambda^{+}$for example.

Coming back to a finite rank free $\Lambda$-chain complex $(\mathcal{C}, d)$, denote by $Z=\operatorname{ker} d \subset \mathcal{C}$ the cycles and by $B=d(\mathcal{C}) \subset \mathcal{C}$ the boundaries. Note that both $Z$ and $B$ are graded $\Lambda$-submodules of $\mathcal{C}$. The following Lemma is an immediate consequence of Lemma 4.4.4 (ii).

Lemma 4.4.6 There exist graded $\Lambda$-submodules $E \subset \mathcal{C}$ and $Z^{\prime} \subset Z$ such that $Z$ and $\mathcal{C}$ split as direct sums of graded $\Lambda$-modules:

$$
Z=Z^{\prime} \oplus d(E), \quad \mathcal{C}=Z^{\prime} \oplus d(E) \oplus E .
$$

In particular, the restriction of $d$ to $E, d_{E}=\left.d\right|_{E}: E \rightarrow d(E)$ is an isomorphism and $d(E)=B$. Moreover, $E \oplus d(E)$ is an acyclic complex and $H_{*}(\mathcal{C}) \cong Z_{*}^{\prime}$.

This decomposition is of course not canonical.

Proof of Lemma 4.4.3 We first show that $\rho$ is injective. Suppose that $\rho(g)=0$. Choose a cycle $\varphi: \mathcal{C}_{*} \rightarrow \Lambda_{*+l}$ representing $g$. As $\rho(g)=0$ we have $\left.\varphi\right|_{Z^{\prime}}=0$ and since $\varphi$ is a cycle we also have $\left.\varphi\right|_{d(E)}=0$. Define $\psi: \mathcal{C}_{*} \rightarrow \Lambda_{*+l+1}$ by $\left.\psi\right|_{Z^{\prime}}=\left.\psi\right|_{E}=0$ and $\left.\psi\right|_{d(E)}=\varphi \circ d_{E}^{-1}$. Clearly we have $\psi \circ d=\varphi$ which means that $\varphi$ is a boundary, hence $g=[\varphi]=0$. This shows that $\rho$ is injective.

It remains to show that $\rho$ is surjective. Let $\varphi: H_{*}(\mathcal{C}) \rightarrow \Lambda_{*+l}$ be an element in $\left(\operatorname{hom}_{\Lambda}(H(\mathcal{C}), \Lambda)\right)_{l}$. View $\varphi$ as $\varphi: Z_{*}^{\prime} \rightarrow \Lambda_{*+l}$. Extend $\varphi$ by 0 to $Z^{\prime} \oplus d(E) \oplus E$. Call this extension $\varphi^{\prime}$. Clearly $\varphi^{\prime}$ is a cycle in $\mathcal{C}_{l}^{\odot}$ and $\rho\left[\varphi^{\prime}\right]=\varphi$. This concludes the proof of Lemma 4.4.3 as well as that of Proposition 4.4.1. 
Remark 4.4.7 (a) The relation between the duality above and Poincaré duality is as follows: in case $\mathcal{C}(-)$ in the statement is replaced with the Morse complex $C(f)$ of some Morse function $f: L \rightarrow \mathbb{R}$ (and we take $\mathcal{R}=\mathbb{Z}_{2}$ ) we may define the morphism $\eta: C(f) \rightarrow s^{n}\left(C(f)^{\odot}\right)$ as a composition of two morphisms with the first being the usual comparison morphism $C(f) \rightarrow C(-f)$ and the second $C(-f) \rightarrow s^{n}\left(C(f)^{\odot}\right)$ given by $\operatorname{Crit}(f) \ni x \rightarrow x^{*} \in \operatorname{hom}_{\mathbb{Z}_{2}}\left(C(f), \mathbb{Z}_{2}\right)^{\text {inv }}$. We have the identifications $H_{k}\left(s^{n}\left(C(f)^{\odot}\right)\right)=H_{k-n}\left(C(f)^{\odot}\right)=H^{n-k}(C(f))$ and the morphism $\eta$ described above induces in homology the Poincaré duality map: $H_{k}(L) \rightarrow H^{n-k}(L)$.

(b) Proposition 4.4.1 also shows that $Q H(L)$ together with the bilinear map $\epsilon_{L}(-\circ-)$ is a Frobenius algebra, though not necessarily commutative.

(c) The quantum inclusion, $i_{L}$, the duality map, $\eta$, and the Lagrangian quantum product determine the module structure by the following formula (which extends (6)):

$$
\left\langle h, i_{L}(x \circ y)\right\rangle=\eta(y)(\operatorname{PD}(h) \circledast x)
$$

for $h \in H^{*}\left(M ; \mathbb{Z}_{2}\right), x, y \in Q H_{*}(L ; \mathcal{R})$. Here $\eta(y) \in H_{*}\left(s^{n}\left(\operatorname{hom}_{\mathcal{R}}(\mathcal{C}(L ; \mathcal{R} ; f), \mathcal{R})\right)\right)$ so that it can be evaluated on $Q H_{*}(L ; \mathcal{R})$. As in formula (6), the pairing on the left side is the $\mathcal{R}$-linear extension of the standard Kronecker pairing.

\subsection{Wide Lagrangians and identifications with singular homology}

Let $L \subset(M, \omega)$ be a monotone wide Lagrangian. This means that there exists an isomorphism $Q H_{*}(L) \cong\left(H\left(L ; \mathbb{Z}_{2}\right) \otimes \Lambda\right)_{*}$. However, in general there is no such canonical isomorphism!

To explain this better, denote by $\mathcal{F}=(f, \rho)$ pairs of Morse data. For any two pairs $\mathcal{F}=(f, \rho)$ and $\mathcal{F}^{\prime}=\left(f^{\prime}, \rho^{\prime}\right)$ and any two choices of almost complex structures $J$ and $J^{\prime}$ denote by $\Psi_{\left(\mathcal{F}^{\prime}, J^{\prime}\right),(\mathcal{F}, J)}: H_{*}(\mathcal{C}(L ; \mathcal{F}, J)) \rightarrow H_{*}\left(\mathcal{C}\left(L ; \mathcal{F}^{\prime}, J^{\prime}\right)\right)$ the canonical isomorphism between the pearl homologies (as described at point (e) in Section 3.2). Denote by $\Psi_{\mathcal{F}^{\prime}, \mathcal{F}}^{\text {Morse }}: H_{*}(\mathcal{F}) \rightarrow H_{*}\left(\mathcal{F}^{\prime}\right)$ the canonical isomorphism between the Morse homologies associated to $\mathcal{F}$ and $\mathcal{F}^{\prime}$. From this point of view, $H_{*}\left(L ; \mathbb{Z}_{2}\right) \otimes \Lambda$ is identified with the family of homologies $H_{*}(\mathcal{F}) \otimes \Lambda$ related by the canonical isomorphisms mentioned above. Similarly, the quantum homology $Q H_{*}(L)$ is identified with the family of homologies $H_{*}(\mathcal{C}(L ; \mathcal{F}, J))$ together with the canonical isomorphisms $\Psi_{\left(\mathcal{F}^{\prime}, J^{\prime}\right),(\mathcal{F}, J)}$. Therefore, specifying a map $I: H\left(L ; \mathbb{Z}_{2}\right) \otimes \Lambda \rightarrow Q H(L)$ is equivalent to having a family of maps $I_{(\mathcal{F}, J)}: H(\mathcal{F}) \otimes \Lambda \rightarrow H(\mathcal{C}(L ; \mathcal{F}, J))$ indexed by regular pairs 
$(\mathcal{F}, J)$ such that the following diagram commutes for every two such pairs $(\mathcal{F}, J)$, $\left(\mathcal{F}^{\prime}, J^{\prime}\right)$ :

$$
\begin{aligned}
& (H(\mathcal{F}) \otimes \Lambda)_{*} \quad \stackrel{\Psi_{\mathcal{F}^{\prime}, \mathcal{F}}^{\text {Morse }}}{\longrightarrow} \quad\left(H\left(\mathcal{F}^{\prime}\right) \otimes \Lambda\right)_{*} \\
& I_{(\mathcal{F}, J)} \downarrow \quad \downarrow I_{\left(\mathcal{F}^{\prime}, J^{\prime}\right)} \\
& H_{*}(\mathcal{C}(L ; \mathcal{F}, J)) \stackrel{\Psi_{\left(\mathcal{F}^{\prime}, J^{\prime}\right),(\mathcal{F}, J)}}{\longrightarrow} H_{*}\left(\mathcal{C}\left(L ; \mathcal{F}^{\prime}, J^{\prime}\right)\right)
\end{aligned}
$$

Of course, in order to define such a family of maps it is enough to choose a reference pair $\left(\mathcal{F}_{0}, J_{0}\right)$, define $I_{\left(\mathcal{F}_{0}, J_{0}\right)}$ and then all the other $I_{(\mathcal{F}, J)}$ are uniquely determined.

The point is that, in general, these choices do not lead to a canonical map $I$. To illustrate this, consider for simplicity the case when $L$ admits a perfect Morse function and consider only Morse data $\mathcal{F}=(f, \rho)$ where $f: L \rightarrow \mathbb{R}$ is a perfect Morse function. Write the pearl differential $d$ as $d=d_{0}+d^{\prime}$, where $d_{0}$ is the Morse differential. As $f$ is perfect we have $d_{0}=0$, so that $d=d^{\prime}$. Moreover, since we assume that $L$ is wide, a dimension comparison shows that $d^{\prime}$ must vanish too (for otherwise the rank of $Q H(L)$ would be smaller than that of $H(L) \otimes \Lambda)$. Thus $d=0$ for every pair $(\mathcal{F}, J)$ as above. It follows that

$$
H_{*}(\mathcal{F})=\mathbb{Z}_{2}\left\langle\operatorname{Crit}_{*}(f)\right\rangle, \quad H_{*}(\mathcal{C}(L ; \mathcal{F}, J))=\left(\mathbb{Z}_{2}\langle\operatorname{Crit}(f)\rangle \otimes \Lambda\right)_{*} .
$$

At first glance it seems that a natural isomorphism between the singular and quantum homologies can be defined by $I_{(\mathcal{F}, J)}(x)=x$ for every $x \in \operatorname{Crit}(f)$ for every $(\mathcal{F}, J)$ (with the Morse function in $\mathcal{F}$ being perfect). A more careful inspection shows that if we define the isomorphisms $I_{(\mathcal{F}, J)}$ in this way the diagram (25) might not commute. A close look at the definition of the comparison morphism $\Psi_{\left(\mathcal{F}^{\prime}, J^{\prime}\right),(\mathcal{F}, J)}$ from point (e) in Section 3.2 (see also an alternative description in the proof of Proposition 4.4.1) shows that $\Psi_{\left(\mathcal{F}^{\prime}, J^{\prime}\right),(\mathcal{F}, J)}$ might differ from $\Psi_{\mathcal{F}^{\prime}, \mathcal{F}}^{\text {Morse }}$ by some quantum terms. In fact we have

$$
\Psi_{\left(\mathcal{F}^{\prime}, J^{\prime}\right),(\mathcal{F}, J)}=\Psi_{\mathcal{F}^{\prime}, \mathcal{F}}^{\text {Morse }}+\sum_{i \geq 1} \Phi_{\left(\mathcal{F}^{\prime}, J^{\prime}\right),(\mathcal{F}, J)}^{i} t^{i}
$$

where the term $\Phi^{i}$ maps $\mathbb{Z}_{2}\left\langle\mathrm{Crit}_{*}(f)\right\rangle$ to $\mathbb{Z}_{2}\left\langle\mathrm{Crit}_{*+i N_{L}}\left(f^{\prime}\right)\right\rangle$ and is defined by counting elements in some moduli spaces involving $J$ and $J^{\prime}$-holomorphic disks with total Maslov index $i N_{L}$. (See the precise description in the proof of Proposition 4.4.1 in Section 4.4.) It is not hard to write down examples where some of the quantum terms $\Phi^{i}$ do not vanish (see Biran and Cornea $[13 ; 12]$ ). In fact, this turns out to be the case for the Clifford torus $\mathbb{T}_{\text {clif }} \subset \mathbb{C} P^{n}$ (see Section 6.2). 
Despite the above there are situations in which a canonical isomorphism $Q H(L) \cong$ $H\left(L ; \mathbb{Z}_{2}\right) \otimes \Lambda$ exists, at least in some degrees.

Proposition 4.5.1 Let $L^{n} \subset\left(M^{2 n}, \omega\right)$ be a monotone Lagrangian (not necessarily wide or narrow).

(i) For every $q \geq n-N_{L}+2$ there exists a canonical isomorphism $I: H_{q}\left(L ; \mathbb{Z}_{2}\right) \longrightarrow$ $Q^{+} H_{q}(L)$. Moreover, this isomorphism maps the fundamental class $[L]$ to the unity in $Q^{+} H(L)$.

(ii) If $L$ is not narrow then the isomorphism $I$ from (i) exists also for $q=n-N_{L}+1$.

(iii) If $L$ is wide, the isomorphism $I$ induces a canonical embedding $H_{q}\left(L ; \mathbb{Z}_{2}\right) \otimes$ $\Lambda_{*} \hookrightarrow Q H_{q+*}(L)$ for every $q \geq n-N_{L}+1$. In particular (for wide Lagrangians), if $N_{L} \geq n+1$ we have a canonical isomorphism $\left(H\left(L ; \mathbb{Z}_{2}\right) \otimes \Lambda\right)_{*} \cong$ $Q H_{*}(L)$.

Proof Let $\mathcal{F}=(f, \rho)$ be a pair formed by a Morse function and a Riemannian metric on $L$ and let $J$ be an almost complex structure on $M$ such that the pearl complex $\mathcal{C}^{+}(L ; \mathcal{F}, J)$ as well as the Morse complex $C(\mathcal{F})$ are defined. Throughout the proof we will assume without loss of generality that $f$ has a unique maximum which we denote by $m$.

Write the pearl differential $d$ on $\mathcal{C}^{+}(L ; \mathcal{F}, J)=C(\mathcal{F}) \otimes \Lambda^{+}$as

$$
d=\partial_{0}+\partial_{1} t+\cdots+\partial_{\nu} t^{\nu}
$$

where $\partial_{0}$ is the Morse differential and $\partial_{i}$ is an operator acting as $\partial_{i}: C_{k}(\mathcal{F}) \rightarrow$ $C_{k-1+i N_{L}}(\mathcal{F})$. For degree reasons we have:

$$
C_{\geq n-N_{L}+1}(\mathcal{F})=\mathcal{C}_{\geq n-N_{L}+1}^{+}(L ; f, \rho, J) .
$$

Moreover, $d=\partial_{0}$ on $C_{\geq n-N_{L}+2}(\mathcal{F})$ and $d=\partial_{0}+\partial_{1} t$ on $C_{n-N_{L}+1}(\mathcal{F})$, where $\partial_{1}: C_{n-N_{L}+1}(\mathcal{F}) \rightarrow C_{n}(\mathcal{F})$.

Point (i) now easily follows since $x \in C_{\geq n-N_{L}+2}(\mathcal{F})$ is a $\partial_{0}$-cycle if and only if it is a $d$-cycle and $x$ is a $\partial_{0}$-boundary if and only if it is a $d$-boundary. Therefore, the map

$$
\tilde{I}: C_{q}(\mathcal{F}) \longrightarrow \mathcal{C}_{q}^{+}(L ; \mathcal{F}, J), \quad \tilde{I}(x)=x,
$$

descends to an isomorphism $I$ in homology. As $m$ represents the fundamental class, $I$ clearly sends $[L]$ to the unity of $Q H^{+}(L)$. This completes the proof of (i) except for the canonicity of $I$ which will be proved soon.

We turn to point (ii). We claim again that $x \in C_{n-N_{L}+1}(\mathcal{F})$ is a $\partial_{0}-$ cycle if and only if it is a $d$-cycle and $x$ is a $\partial_{0}$-boundary if and only if it is a $d$-boundary. Indeed, the 
claim is obvious for boundaries since $d=\partial_{0}$ on $C_{n-N_{L}+2}(\mathcal{F})$. It remains to show that $\partial_{0}$ and $d$-cycles coincide on $C_{n-N_{L}+1}(\mathcal{F})$. Let $x \in C_{n-N_{L}+1}(\mathcal{F})$. We have $d(x)=\partial_{0}(x)+\partial_{1}(x) t$. This implies that if $x$ is a $d$-cycle then it is also a $\partial_{0}$-cycle. Suppose now that $x$ is a $\partial_{0}$-cycle. We then have $d(x)=\partial_{0}(x)+\partial_{1}(x) t=\partial_{1}(x) t$. If $d(x) \neq 0$ then $d(x)=m t$ which implies that $m$ is a boundary hence $Q H(L)=0$ and $L$ is narrow, contrary to our assumption. Thus $d(x)=0$ and $x$ is a $d$-cycle.

We can now extend the definition of $\tilde{I}$ to $\tilde{I}: C_{n-N_{L}+1}(\mathcal{F}) \longrightarrow \mathcal{C}_{n-N_{L}+1}^{+}(L ; \mathcal{F}, J)$ by $\widetilde{I}(x)=x$, and as before $\tilde{I}$ descends to an isomorphism $I$ in homology.

To conclude the proofs of points (i), (ii) it remains to show that $I$ is canonical in the sense discussed before the statement of the proposition. To see this, write the map $\tilde{I}$ as $\tilde{I}_{(\mathcal{F}, J)}$ to denote the relation to the data $(\mathcal{F}, J)$. For degree reasons it follows that the maps $\Phi_{\left(\mathcal{F}^{\prime}, J^{\prime}\right),(\mathcal{F}, J)}^{i}$ in (26) vanish on $\mathcal{C}_{q}^{+}$for $q \geq n-N_{L}+1$, hence the squares in (25) commute. This completes the proof of the first point of the proposition.

We now prove (iii). Consider the canonical map $p: Q^{+} H(L) \rightarrow Q H(L)$ induced by the extension of coefficients $\Lambda^{+} \rightarrow \Lambda$. The embedding $H_{q}\left(L ; \mathbb{Z}_{2}\right) \otimes \Lambda_{*} \hookrightarrow Q H_{q+*}(L)$ is induced by the map $p \circ I: H_{q}\left(L ; \mathbb{Z}_{2}\right) \rightarrow Q H_{q}(L)$. So, the proof is reduced to showing that $p \circ I$ is an injection. To see this, let $x \in C_{q}(\mathcal{F})$ be a $\partial_{0}$-cycle with nontrivial Morse homology class $[x]_{\text {Morse }}$, where $q \geq n-N_{L}+1$. By what we have just proved, $x$ is also a $d$-cycle. We have to prove that $x$, when viewed as an element in $\mathcal{C}_{q}(L ; \mathcal{F}, J)$, is not a $d$-boundary. Consider the minimal model $\mathcal{C}_{\text {min }}(L)$ together with the structural map $\phi: \mathcal{C}(L ; \mathcal{F}, J) \rightarrow \mathcal{C}_{\min }(L)$ as constructed in Section 4.1. Recall that by that construction $\phi_{0}(x)=[x]_{\text {Morse }} \neq 0$, hence $\phi(x) \neq 0$. On the other hand, by Remark 4.1.3 (c), the differential of $\mathcal{C}_{\min }(L)$ vanishes because $L$ is wide. As $\phi$ is a chain map it follows that $x$ cannot be a $d$-boundary.

\section{Proofs of the main theorems}

This section is focused on proving the three main theorems of the introduction.

Before we go on with the proof we would like to make a small but useful algebraic observation which will be used many times in the sequel. Consider the graded vector space $H\left(L ; \mathbb{Z}_{2}\right) \otimes \Lambda^{+}$endowed with the grading coming from both factors. Let $a \in\left(H\left(L ; \mathbb{Z}_{2}\right) \otimes \Lambda^{+}\right)_{l}$ be a homogeneous element (of degree $l$ ). Then we can decompose $a$ in a unique way as

$$
a=\sum_{r \geq 0} a_{l+r N_{L}} t^{r}, \quad a_{l+r N_{L}} \in H_{l+r N_{L}}\left(L ; \mathbb{Z}_{2}\right) .
$$


Suppose now that $a_{l+r_{0} N_{L}}=[L] \in H_{n}\left(L ; \mathbb{Z}_{2}\right)$ for some $r_{0}$. In that case we will say that $a$ contains $[L] t^{r_{0}}$. (Note that this can happen only if $l+r_{0} N_{L}=n$.) Then, as $H_{>n}\left(L ; \mathbb{Z}_{2}\right)=0,|t|<0$, the decomposition of $a$ cannot contain terms with $t$ of higher order than $r_{0}$, ie

$$
a=[L] t^{r_{0}}+a_{n-N_{L}} t^{r_{0}-1}+a_{n-2 N_{L}} t^{r_{0}-2}+\cdots .
$$

We will abbreviate this by writing $a=[L] t^{r_{0}}+$ 1.o. $(t)$, where 1.o. $(t)$ stands for terms of lower order in $t$. Similarly, if a homogeneous element $a$ contains $[\mathrm{pt}] t^{l_{0}}$ for some $l_{0} \geq 0$, then we must have $a=[\mathrm{pt}] t^{l_{0}}+$ h.o. $(t)$, where h.o. $(t)$ stands for terms of higher order in $t$.

A similar discussion applies to homogeneous elements in the positive quantum homology $Q H_{*}\left(M ; \Lambda^{+}\right)=\left(H\left(M ; \mathbb{Z}_{2}\right) \otimes \Lambda^{+}\right)_{*}$, as well as in the positive pearl complex $\mathcal{C}^{+}(L ; f, \rho, J)$ in case the function $f$ has a unique maximum and a unique minimum.

\subsection{Proof of Theorem 1.2.2}

The argument is based on the minimal model machinery from Section 4.1. Consider the pearl complex $\mathcal{C}^{+}(f, J)$ and recall from Section 4.1 that there exists a chain complex $\left(\mathcal{C}_{\min }^{+}(L)=H\left(L ; \mathbb{Z}_{2}\right) \otimes \Lambda^{+}, \delta\right)$, unique up to isomorphism, and chain morphisms $\phi: \mathcal{C}^{+}(f, J) \rightarrow \mathcal{C}_{\min }^{+}(L), \psi: \mathcal{C}_{\min }^{+}(L) \rightarrow \mathcal{C}^{+}(f, J)$ so that $\phi \circ \psi=\mathrm{id}, \delta_{0}=0$ (where $\delta_{0}$ is obtained from $\delta$ by putting $t=0$ ) and $\phi, \psi, \phi_{0}, \psi_{0}$ induce isomorphisms in quantum and Morse homologies. By Remark 4.1.3 the quantum product in $\mathcal{C}^{+}(f, J)$ can be transported by the morphisms $\phi$ and $\psi$ to a product $*: \mathcal{C}_{\text {min }}^{+}(L) \otimes \mathcal{C}_{\text {min }}^{+}(L) \rightarrow \mathcal{C}_{\text {min }}^{+}(L)$ which is a chain map and a quantum deformation of the singular intersection product and so that $[L] \in H_{n}\left(L ; \mathbb{Z}_{2}\right)$ is the unity at the chain level (notice though that, as the maps $\phi$ and $\psi$ are not canonical, this product is not canonical either). As discussed before we put $\mathcal{C}_{\text {min }}(L)=\mathcal{C}_{\text {min }}^{+}(L) \otimes_{\Lambda^{+}} \Lambda=H\left(L ; \mathbb{Z}_{2}\right) \otimes \Lambda$. As in the statement of the theorem we assume that $H_{*}\left(L ; \mathbb{Z}_{2}\right)$ is generated by $H_{\geq n-l}\left(L ; \mathbb{Z}_{2}\right)$. In view of Remark 4.1.3 (b) the first point of the theorem reduces to the next lemma.

Lemma 5.1.1 Suppose that $N_{L} \geq l+1$. If $\delta$, the differential of the minimal pearl complex, does not vanish, then $[L]$ is a boundary in $\mathcal{C}_{\min }(L), Q H(L)=0$, and $N_{L}=$ $l+1$.

Proof There are two possibilities: either $\delta=0$ on $H_{n-l}\left(L ; \mathbb{Z}_{2}\right)$, or $\delta \neq 0$ on that homology.

Assume first that $\delta=0$ on $H_{n-l}\left(L ; \mathbb{Z}_{2}\right)$. We claim that $\delta=0$ everywhere. To show this we will prove by induction that $\delta=0$ on $H_{\geq n-l-s}\left(L ; \mathbb{Z}_{2}\right)$ for every $s \geq 0$. 
Indeed, for $s=0$ this is true since $N_{L} \geq l+1$ implies that $\delta=0$ on $H_{\geq n-l+1}\left(L ; \mathbb{Z}_{2}\right)$, and moreover we have assumed that $\delta=0$ on $H_{n-l}\left(L ; \mathbb{Z}_{2}\right)$. Assume now that the assertion is true for some $s \geq 0$ and let $x \in H_{\geq n-l-s-1}\left(L ; \mathbb{Z}_{2}\right)$. By the assumptions of Theorem 1.2.2 we can write $x=\sum_{j} a_{j}$ where each $a_{j}$ is expressed as (classical) intersection products of elements from $H_{\geq n-l}\left(L ; \mathbb{Z}_{2}\right)$. We now claim that $\delta\left(a_{j}\right)=0$ for every $j$. To see this write $a_{1}=x_{1} \cdots x_{r}$ with $x_{i} \in H_{\geq n-l}\left(L ; \mathbb{Z}_{2}\right)$, where $-\cdot-$ is the classical intersection product. We then have $\delta\left(x_{i}\right)=0$ and we write $\delta\left(x_{1} * x_{2} * \cdots * x_{r}\right)=\sum_{i} x_{1} * \cdots \delta\left(x_{i}\right) * \cdots * x_{r}=0$. At the same time

$$
x_{1} * x_{2} * \cdots * x_{r}=a_{1}+\sum_{q>0} z_{q} t^{q},
$$

with $z_{j} \in H_{\geq n-l-s}\left(L ; \mathbb{Z}_{2}\right)$. (Recall that $\left.|t|=-N_{L} \leq-2\right)$. By the induction hypothesis we have $\delta\left(z_{j}\right)=0$, hence $\delta\left(a_{1}\right)=0$. The same argument shows that $\delta\left(a_{j}\right)=0$ for every $j$. It follows that $\delta(x)=0$. This proves that $\delta=0$ on $H_{\geq n-l-s-1}\left(L ; \mathbb{Z}_{2}\right)$ and completes the induction.

We now turn to the second case: $\delta \neq 0$ on $H_{n-l}\left(L ; \mathbb{Z}_{2}\right)$. First note that we must have $N_{L}=l+1$. Indeed, if $N_{L} \geq l+2$ then by degree reasons $\delta=0$ on $H_{n-l}\left(L ; \mathbb{Z}_{2}\right)$ and by what we have just proved we obtain $\delta=0$ everywhere, a contradiction. Thus $N_{L}=l+1$. By degree reasons again it follows that $\delta$ sends $H_{n-l}\left(L ; \mathbb{Z}_{2}\right)$ nontrivially to $H_{n}\left(L ; \mathbb{Z}_{2}\right) t$. Thus there exists $x \in H_{n-l}\left(L ; \mathbb{Z}_{2}\right)$ such that $\delta(x)=[L] t$. This implies that $[L]$ is a boundary. As $[L]$ is the unity of $Q H(L)$ we also obtain $Q H(L)=0$.

We now pursue with the proof of the second point of Theorem 1.2.2. Thus we assume that $L$ is narrow and so $[L]$ is a boundary in $\mathcal{C}_{\min }(L)$ and $N_{L} \leq l+1$. Let $K$ be the constant in the statement of the theorem, $K=\max \left\{l+1, n+1-N_{L}\right\}$ when $N_{L}<l+1$ and $K=l+1$ when $N_{L}=l+1$. Notice that the degree $n$ component of $\mathcal{C}_{\min }^{+}(L)$ is one-dimensional. This implies that, despite the fact that the minimal pearl model is determined only up to a noncanonical isomorphism, the generator in degree $n$ is canonical. It will be denoted (as before) by $[L]$.

In the following lemma we denote the differential of the complex $\mathcal{C}_{\min }^{+}(L)$ by $\delta^{+}$to distinguish it from its extension $\delta=\delta^{+} \otimes 1$ defined on $\mathcal{C}_{\min }(L)=\mathcal{C}_{\min }^{+}(L) \otimes_{\Lambda^{+}} \Lambda$. The main step is:

Lemma 5.1.2 Either there exists some $x \in H_{*}\left(L ; \mathbb{Z}_{2}\right)$ so that $\delta^{+}(x)=[L] t^{q}+$ 1.o.( $(t)$ or there are $y, z \in H_{*}\left(L ; \mathbb{Z}_{2}\right)$ so that $y * z=[L] t^{q}+$ l.o. $(t)$, where in both cases $0<q N_{L} \leq K$.

Proof As $L$ is narrow, the first point of Theorem 1.2.2 implies that $N_{L} \leq l+1$. Assume first that $N_{L}=l+1$. Then by definition $K=l+1$. In this case, as the proof 
of Lemma 5.1.1 shows, there exists $x \in H_{n-l}\left(L ; \mathbb{Z}_{2}\right)$ such that $\delta^{+} x=[L] t$. Thus $x$ satisfies the statement of our lemma with $q=1$.

We will assume from now on that $N_{L}<l+1$, and so $K \geq l+1, n+1-N_{L}$. Let $w \in H_{*}\left(L ; \mathbb{Z}_{2}\right)$ be an element of maximal degree so that $\delta^{+} w$ contains $[L] t^{s}$ for some $s>0$. More precisely, denote by $\rho \in H^{n}\left(L ; \mathbb{Z}_{2}\right)$ the generator (so that $\langle\rho,[L]\rangle=1$ ). We require that $w$ is of maximal degree so that $\left\langle\rho, \delta^{+} w\right\rangle \neq 0 \in \Lambda^{+}$. (Here and in what follows we extend the Kronecker pairing $\langle\cdot, \cdot\rangle$ to $H_{*}\left(L ; \mathbb{Z}_{2}\right) \otimes \Lambda^{+}$by linearity over $\Lambda^{+}$.) Note that such a $w$ must exist, since $L$ is narrow hence $[L] t^{r}$ must be a $\delta^{+}$-boundary for some $r \geq 1$.

If $|w| \geq n-l$, the statement of our lemma is satisfied with $x=w$ and $q=s$ because $q N_{L}=n-\left|\delta^{+} w\right|=n+1-|w| \leq l+1 \leq K$. Therefore we assume from now on that $|w|<n-l$. We know that $H_{\geq n-l}\left(L ; \mathbb{Z}_{2}\right)$ generates $H_{*}\left(L ; \mathbb{Z}_{2}\right)$ as an algebra. In particular, $|w|<n-l$ implies that $w$ is decomposable with respect to the intersection product. We now write

$w=w_{1} \cdot w_{2}=w_{1} * w_{2}+\sum_{i>0} z_{i} t^{i}, \quad$ with $|w|<\left|w_{1}\right|<n,|w|<\left|w_{2}\right|<n,|w|<\left|z_{i}\right|$.

(Of course, $w$ can be a sum of such products but this does not make any difference in the argument and, in terms of notation, it is simpler to assume that just one such monomial appears.) Now

$$
\left\langle\rho, \delta^{+} w\right\rangle=\left\langle\rho,\left(\delta^{+} w_{1}\right) * w_{2}+w_{1} *\left(\delta^{+} w_{2}\right)\right\rangle+\sum_{i>0}\left\langle\rho, z_{i}\right\rangle t^{i}
$$

By the maximality of $|w|$, and the fact that $|w|<\left|z_{i}\right|$, we see that the second term on the right vanishes and we also get that for either $w_{1}$ or $w_{2}$, say $w_{1}$ (the other case is similar) we have $\left\langle\rho,\left(\delta^{+} w_{1}\right) * w_{2}\right\rangle=t^{q^{\prime}}$ for some $q^{\prime}>0$. We now write $\delta^{+} w_{1}=\sum_{i>0} u_{i} t^{i}$ and we deduce that for some $i>0$ we have $\left\langle\rho, u_{i} * w_{2}\right\rangle=t^{q^{\prime}-i}$. Notice that $\left|u_{i}\right|=\left|w_{1}\right|+i N_{L}-1$. We put $q=q^{\prime}-i$ (clearly $q \geq 0$ and we will show below that $q>0$ ). We now get

$$
\begin{aligned}
n-q N_{L}=\left|u_{i} * w_{2}\right|=\left|u_{i}\right|+\left|w_{2}\right|-n & =\left|w_{1}\right|+i N_{L}-1+\left|w_{2}\right|-n \\
& =|w|+i N_{L}-1 \geq N_{L}-1 .
\end{aligned}
$$

Thus, $q N_{L} \leq n-N_{L}+1 \leq K$ and the statement of our lemma will be satisfied with $y=u_{i}$ and $z=w_{2}$.

It remains only to check that $q>0$. Assume by contradiction that $q=0$, or equivalently that $q^{\prime}=i$. This implies that $u_{i} * w_{2}=[L]$. But for degree reasons this cannot happen since $\left|w_{2}\right|<n$. A contradiction. 
To prove the second point of Theorem 1.2.2 we will use Lemma 5.1.2 to show that $L$ is uniruled of order $K$. For this, we fix a generic almost complex structure $J$ as well as a point $P \in L$. Fix a Morse function $f$ and a Riemannian metric $\rho_{L}$ on $L$ so that the pair $\left(f, \rho_{L}\right)$ is Morse-Smale. Moreover, we choose $f$ so that $P$ is its unique maximum. We also pick a second Morse function $f_{1}$ so that the pair $\left(f_{1}, \rho_{L}\right)$ is also Morse-Smale, and $f$ and $f_{1}$ are in general position. We assume that $J$ is generically chosen so that $\mathcal{C}^{+}\left(L ; f, \rho_{L}, J\right)$ and $\mathcal{C}^{+}\left(L ; f_{1}, \rho_{L}, J\right)$ are both defined as well as the relevant product. As above, we let $\mathcal{C}_{\text {min }}^{+}(L)$ be the minimal pearl complex and we fix $\phi, \psi, \phi_{1}, \psi_{1}$, the structure maps associated to $\left(f, \rho_{L}, J\right)$ and, respectively, to $\left(f_{1}, \rho_{L}, J\right)$ as constructed in the proof of Proposition 4.1.1 in Section 4.1.

The following technical result is an easy consequence of the proof of Proposition 4.1.1 and is valid independently of whether $L$ is narrow or not.

Lemma 5.1.3 (i) If there exists $z \in \operatorname{Crit}(f)$ so that $\phi(z)=[L] t^{s}+$ 1.o. $(t), s>0$, then there exists $w \in \operatorname{Crit}(f)$ so that $d w=P t^{s^{\prime}}+$ 1.o. $(t)$ with $0<s^{\prime} \leq s$.

(ii) Let $a \in H_{*}\left(L ; \mathbb{Z}_{2}\right)$ be a homogeneous element such that $\psi(a)=P t^{s}+$ 1.o. $(t)$, $s>0$. Then there exists $w \in \operatorname{Crit}(f)$ such that $d w=P t^{s^{\prime}}+$ 1.o.( $(t)$ with $0<s^{\prime}<s$.

Proof We begin with point (i). As in the proof of Proposition 4.1.1, change the basis in $\mathbb{Z}_{2}\langle\operatorname{Crit}(f)\rangle$ so that the generators forming the new basis are of three types $B_{X}, B_{Y} \subset \operatorname{ker}\left(d_{0}\right)$ and $B_{Y^{\prime}}$ so that $B_{Y}$ and $B_{Y^{\prime}}$ are in bijection and $d_{0}\left(B_{Y^{\prime}}\right)=$ $B_{Y}$ (where $d_{0}$ is the Morse differential). For $y \in B_{Y}$ we denote by $y^{\prime} \in B_{Y^{\prime}}$ the element so that $d_{0}\left(y^{\prime}\right)=y$. As $\mathcal{C}_{n}^{+}(L ; f, \rho, J)=\mathbb{Z}_{2} P$ we have $P \in B_{X}$. The map $\phi: \mathcal{C}^{+}(L ; f, \rho, J) \rightarrow \mathcal{C}_{\min }^{+}(L)$ is defined so that for $x \in B_{X}, \phi(x)=[x]([x]$ is the Morse homology class of $x$ ), for $y^{\prime} \in B_{Y^{\prime}}, \phi\left(y^{\prime}\right)=0$ and for $y \in B_{Y}$, $\phi(y)=\phi\left(y-d y^{\prime}\right)$. Let $u \in B_{Y}$ be a generator of the highest degree among the elements of $B_{Y}$ with the property that there exists $0<s^{\prime} \leq s$ with $\phi(u)=[L] t^{s^{\prime}}+$ l.o. $(t)$ (since $\phi(x)=[x]$ for $x \in B_{X}, \phi\left(y^{\prime}\right)=0$ for $y^{\prime} \in B_{Y^{\prime}}$ and $\phi(z)=[L] t^{s}+$ l.o. $(t)$ with $s>0$, there must be such a $u$ ). Write

$$
u-d u^{\prime}=\sum_{i>0} x_{i} t^{i}+\sum_{j>0} y_{j} t^{j}+\sum_{k>0} y_{k}^{\prime} t^{k}, \quad \text { with } x_{i} \in B_{X}, y_{j} \in B_{Y}, y_{k}^{\prime} \in B_{Y^{\prime}} .
$$

We now have

$$
[L] t^{s^{\prime}}+\text { 1.o. }(t)=\phi(u)=\phi\left(u-d u^{\prime}\right)=\sum_{i>0} \phi\left(x_{i}\right) t^{i}+\sum_{j>0} \phi\left(y_{j}\right) t^{j} .
$$

Note that $\left|y_{j}\right|>|u|$ and therefore by the maximality of $u$ none of the terms $\phi\left(y_{j}\right)$ can contribute an $[L] t^{s^{\prime \prime}}, s^{\prime \prime}>0$ to that sum. Moreover, none of the terms $\phi\left(y_{j}\right)$ 
can contribute $[L]$ to that sum since $\phi$ of an element in $B_{Y}$ is divisible by $t$. It follows that there exists $i_{0}$ such that the term $\phi\left(x_{i_{0}}\right) t^{i_{0}}$ contributes the element $[L] t^{s^{\prime}}$. As $\phi\left(x_{i_{0}}\right)=\left[x_{i_{0}}\right]_{\text {Morse }}$ it follows that $x_{i_{0}}=P$ and $i_{0}=s^{\prime}$. As the degree $n$ part of $B_{X}$ is $P$, and $B_{Y}, B_{Y^{\prime}}$ do not contain elements of degree $n$, it follows that $u-d u^{\prime}=P t^{s^{\prime}}+$ l.o. $(t)$. As $u$ is a linear combination of pure critical points (it doesn't involve $t$ 's) we now obtain that $d u^{\prime}=P t^{s^{\prime}}+$ l.o. $(t)$ (we work here over $\mathbb{Z}_{2}$ so $P=-P$ ). Finally, there must be a critical point $w$ participating in $u^{\prime}$ (which is a linear combination of critical points) so that $d w=P t^{s^{\prime}}+$ l.o. $(t)$. This completes the proof of point (i).

We turn to the proof of (ii). Write

$$
\psi(a)=P t^{s}+z_{s-1} t^{s-1}+\cdots+z_{1} t+z_{0},
$$

with $z_{i} \in \mathbb{Z}_{2}\langle\operatorname{Crit}(f)\rangle$. Note that $z_{0}=\psi_{0}(a)$ and that by the construction of $\phi$ and $\psi$ in the proof of Proposition 4.1.1 in Section 4.1 we also have $\phi\left(z_{0}\right)=a$. Recall also that $\phi \circ \psi=$ id. Using this, and applying $\phi$ to both sides of (29) we obtain

$$
0=[L] t^{s}+\phi\left(z_{s-1}\right) t^{s-1}+\cdots+\phi\left(z_{1}\right) t .
$$

Clearly not all of $\left\langle\rho, \phi\left(z_{1}\right)\right\rangle, \ldots,\left\langle\rho, \phi\left(z_{s-1}\right)\right\rangle$ can vanish (where, as before, $\rho \in$ $H^{n}\left(L ; \mathbb{Z}_{2}\right)$ is the generator). Let $1 \leq j \leq s-1$ be an index such that $\left\langle\rho, \phi\left(z_{j}\right)\right\rangle \neq 0$. We then have $\phi\left(z_{j}\right)=[L] t^{s-j}+$ l.o. $(t)$. By point (i) just proved, there exists $w$ and $0<s^{\prime} \leq s-j<s$ such that $d w=P t^{s^{\prime}}+$ 1.o. $(t)$.

We continue with the proof of point (ii) of Theorem 1.2.2. We begin by analyzing the first possibility resulting from Lemma 5.1.2: $\delta^{+} x=[L] t^{q}+$ 1.o. $(t)$ for some $x \in H_{*}\left(L ; \mathbb{Z}_{2}\right)$ with $0<q N_{L} \leq K$.

Consider the map $\phi: \mathcal{C}^{+}\left(L ; f, \rho_{L}, J\right) \rightarrow \mathcal{C}_{\text {min }}^{+}(L)$. As the degree $n$ part of $B_{X}$ consists of $P$ only, we have $\phi(P)=[L]$. By the definition of $\phi$ there exists $u \in \mathbb{Z}_{2}\left\langle B_{X}\right\rangle$ such that $\phi(u)=x$. Write $d u=\sum_{i \geq 0} a_{i} t^{i}$. We have: $[L] t^{q}+$ l.o. $(t)=\delta^{+} x=\delta^{+} \phi(u)=$ $\phi(d u)=\sum_{i \geq 0} \phi\left(a_{i}\right) t^{i}$. Thus there exists $0 \leq j \leq q$ such that $\phi\left(a_{j}\right) t^{j}=[L] t^{q}+$ l.o. $(t)$. There are two possibilities: either $j=q$ or $j<q$. In case $j=q$ we must have $\phi\left(a_{j}\right)=[L]$ hence $a_{j}=P$ and it follows that $d u=P t^{q}+$ l.o. $(t)$. The element $u$ might not be a single critical point of $f$ but a linear combination of such. However there must be a critical point $w$ participating in the linear combination $u$ such that $d w=P t^{q}+$ l.o. $(t)$. In case $j<q$ we obtain $\phi\left(a_{j}\right)=[L] t^{q-j}+$ l.o. $(t)$ and as $q-j>0$ we deduce from Lemma 5.1.3 that there exists $w \in \operatorname{Crit}(f)$ so that $d w=P t^{q^{\prime}}+$ 1.o.( $(t)$ with $0<q^{\prime} \leq q-j$. Summarizing, we see that in both cases $(j=q$ and $j<q)$ there is $w \in \operatorname{Crit}(f)$ so that $d w=P t^{s}+$ l.o. $(t)$ with $0<s \leq q$. This implies that there exists a nonconstant $J$-disk through $P$ of Maslov index at most $q N_{L}$. 
It remains to discuss the second case: $y * z=[L] t^{q}+$ l.o. $(t)$. The argument is similar. By definition $y * z=\phi\left(\psi_{1}(y) * \psi(z)\right)$. Write $\psi_{1}(y)=\sum_{i \geq 0} y_{i} t^{i}, \psi(z)=\sum_{j \geq 0} z_{j} t^{j}$, with $y_{i} \in \mathbb{Z}_{2}\left\langle\operatorname{Crit}\left(f_{1}\right)\right\rangle, z_{j} \in\langle\operatorname{Crit}(f)\rangle$ being homogeneous elements. The equality $[L] t^{q}+$ l.o. $(t)=\sum_{i, j} \phi\left(y_{i} * z_{j}\right) t^{i+j}$ implies that there exist $i, j \geq 0$ such that $\phi\left(y_{i} * z_{j}\right) t^{i+j}=[L] t^{q}+$ l.o. $(t)$. Write $y_{i} * z_{i}=\sum_{k \geq 0} p_{k} t^{k}$. We get that there exists $k \geq 0$ such that $\phi\left(p_{k}\right) t^{k+i+j}=[L] t^{q}+$ l.o. $(t)$. Now there are two possibilities: either $k+i+j<q$ or $k+i+j=q$.

In the first case $(k+i+j<q)$ we get $\phi\left(p_{k}\right)=[L] t^{q-(k+i+j)}+$ 1.o. $(t)$ and so by Lemma 5.1.3 (i) there exists $w \in \operatorname{Crit}(f)$ such that $d w=P t^{s^{\prime}}+$ 1.o.( $(t)$ with $0<s^{\prime} \leq q-(k+i+j)$. It follows that there exists a nonconstant $J$-disk through $P$ with Maslov index $\leq s^{\prime} N_{L} \leq q N_{L}$.

In the second case $(k+i+j=q)$ we have $\phi\left(p_{k}\right)=[L]$ hence $p_{k}=P$ and $y_{i} * z_{j}=P t^{k}+$ 1.o. $(t)$. If $k>0$ there exists a nonconstant $J$-disk through $P$ with Maslov index $\leq k N_{L} \leq q N_{L}$. In case $k=0$ we have $y_{i} * z_{j}=P$ hence for degree reasons $z_{j}=P$ (and $y_{i}=P_{1}$, where $P_{1}$ is the maximum of $f_{1}$ ). It follows that $\psi(z)=P t^{j}+$ 1.o. $(t)$. We have $j>0$, for otherwise $\psi(z)=P$ so $z=[L]$ which is impossible in view of our starting equality $y * z=[L] t^{q}+$ l.o. $(t)$ with $y \in H_{*}\left(L ; \mathbb{Z}_{2}\right)$ and $q>0$. Thus $\psi(z)=P t^{j}+$ 1.o. $(t)$ with $0<j \leq q$. By Lemma 5.1 .3 (ii) there exists $w \in \operatorname{Crit}(f)$ with $d w=P t^{j^{\prime}}+$ l.o. $(t)$ with $0<j^{\prime}<j \leq q$ and it follows that there exists a nonconstant $J$-disk through $P$ with Maslov index $\leq j^{\prime} N_{L}<q N_{L}$. This concludes the proof of Theorem 1.2.2.

\subsection{Proof of Theorem 1.2.3}

Recall that we now suppose that $M$ is point invertible of order $k$. This means that in the quantum homology of $M$ with coefficients in $\Gamma^{+}=\mathbb{Z}_{2}[s]$ there exists $a \in Q H_{*}\left(M ; \Gamma^{+}\right), a=a_{0}+a_{1} s$ with $0 \neq a_{0} \in H_{*}\left(M ; \mathbb{Z}_{2}\right)$ and $a_{1} \in Q H_{*}\left(M ; \Gamma^{+}\right)$ so that $[\mathrm{pt}] * a=[M] s^{k / 2 C_{M}}$. Recall that here $|s|=-2 C_{M}$. Denote $Q H\left(M ; \Lambda^{+}\right)=$ $Q H(M) \otimes_{\mathbb{Z}_{2}[s]} \Lambda^{+}$. Clearly, we also have in $Q H\left(M ; \Lambda^{+}\right)$, [pt] $* a=[M] t^{k / N_{L}}$.

We start with the point (i) of the theorem. We first notice that the relation [pt] $* a=$ $[M] t^{k / N_{L}}$ implies $|a|-2 n=2 n-k$ and as $a=a_{0}+a_{1} s$ we have $0 \leq|a| \leq 2 n$ and so $k=4 n-|a| \geq 2 n$. We now use the module structure

$$
Q H\left(M ; \Lambda^{+}\right) \otimes Q^{+} H(L) \rightarrow Q^{+} H(L)
$$

to write

(30) $a *([\mathrm{pt}] *[L])=(a *[\mathrm{pt}]) *[L]=([\mathrm{pt}] * a) *[L]=[M] *[L] t^{k / N_{L}}=[L] t^{k / N_{L}}$. 
We need to analyze Equation (30) at the chain level. For this, we fix a Morse function $f: L \rightarrow \mathbb{R}$ with a single maximum $P_{f}$ as well as a Morse function $g: M \rightarrow \mathbb{R}$ with a single maximum $P_{g}$ and a single minimum $m_{g}$. We also fix Riemannian metrics $\rho_{L}$ and $\rho_{M}$ on $L$ and $M$. The Morse complex of $g$ tensored with $\Lambda^{+}$will be denoted by $C^{+}(g)$. We also fix a minimal pearl complex for $L, \mathcal{C}_{\text {min }}^{+}(L)$, together with the two associated structural maps $\phi$ and $\psi$ as in Section 4.1. We use the module operation (on the chain level) in the form

$$
C^{+}(g) \otimes \mathcal{C}_{\min }^{+}(L) \rightarrow \mathcal{C}_{\min }^{+}(L),
$$

by transporting the module operation $C^{+}(g) \otimes \mathcal{C}^{+}\left(L ; f, \rho_{L}, J\right) \rightarrow \mathcal{C}^{+}\left(L ; f, \rho_{L}, J\right)$ via the structural maps $\phi, \psi$, ie for $h \in C^{+}(g), \alpha \in \mathcal{C}_{\text {min }}^{+}(L)$ we define $h * \alpha=\phi(a * \psi(\alpha))$. We write

$$
y=m_{g} *[L]=\sum_{i>0} z_{i} t^{i}, \quad \text { where } z_{i} \in H_{*}\left(L ; \mathbb{Z}_{2}\right) .
$$

Note that there are no classical terms here (ie $i=0$ ) for degree reasons, since $|y|=-n$.

Lemma 5.2.1 There exists $0<i<k / N_{L}$ such that $z_{i} \neq 0$.

Proof We write $y$ as a sum of three terms: $y=S_{1}+z^{\prime} t^{k / N_{L}}+S_{2}$ with

$$
S_{1}=\sum_{i=1}^{k / N_{L}-1} z_{i} t^{i}, \quad S_{2}=\sum_{i \geq k / N_{L}+1} z_{i} t^{i}
$$

and $z_{i}, z^{\prime} \in H_{*}\left(L ; \mathbb{Z}_{2}\right)$. Notice that $S_{2}=0$ because $k \geq 2 n,|y|=-n,\left|z_{i}\right| \leq n$.

Choose a cycle $a^{\prime} \in C^{+}(g)$ which represents $a$. We have

$$
a^{\prime} * y=a^{\prime} * S_{1}+a^{\prime} * z^{\prime} t^{k / N_{L}}
$$

and thus, $a^{\prime} * S_{1}+\left(a^{\prime} * z^{\prime}-[L]\right) t^{k / N_{L}} \in \operatorname{Im}\left(\delta^{+}\right)$.

We now claim that $a^{\prime} * z^{\prime}=0$. To see this, first note that $\left|a^{\prime} * z^{\prime}\right|=|a|+\left|z^{\prime}\right|-2 n=$ $(4 n-k)+(-n+k)-2 n=n$. Write $a^{\prime} * z^{\prime}=\sum_{q \geq 0} b_{q} t^{q}$, with $b_{q} \in H_{*}\left(L ; \mathbb{Z}_{2}\right)$. We have $\left|b_{q}\right|=\left|a^{\prime} * z^{\prime}\right|+q N_{L}=n+q N_{L}$, hence $b_{q}=0$ for every $q \geq 1$. Thus $a^{\prime} * z^{\prime}=b_{0}$. Assume by contradiction that $b_{0} \neq 0$. Then $\left|a^{\prime}\right|=n+2 n-\left|z^{\prime}\right| \geq 2 n$ and so $\left|a^{\prime}\right|=2 n$, hence $a^{\prime}=P_{f}$ and $a=[M]$. This is impossible in view of our assumption that $[\mathrm{pt}] * a=[M] t^{k / N_{L}}$. This proves that $a^{\prime} * z^{\prime}=0$.

We now have

$$
a^{\prime} * S_{1}-[L] t^{k / N_{L}} \in \operatorname{Im}\left(\delta^{+}\right)
$$


From this equality we deduce $S_{1} \neq 0$ and the statement of the Lemma. Indeed, if $S_{1}=0$, then $[L]$ is a boundary in $\mathcal{C}_{\min }(L ; \Lambda)$ which implies that $L$ is narrow, contradicting our assumption.

We continue with the proof of point (i) of Theorem 1.2.3. In view of Lemma 5.2.1 choose the minimal index $0<i_{0}<k / N_{L}$ such that $z_{i_{0}} \neq 0$. We have $m_{g} *[L]=$ $z_{i_{0}} t^{i_{0}}+$ h.o. $(t)$. (h.o. $(t)$ stands for higher order terms in $t$.) We now have: $\phi\left(m_{g} *\right.$ $\psi([L]))=z_{i_{0}} t^{i_{0}}+$ h.o. $(t)$. But $\psi([L])=P_{f}$, hence $\phi\left(m_{g} * P_{f}\right)=z_{i_{0}} t^{i_{0}}+$ h.o. $(t)$. Note that the classical term in $m_{g} * P_{f}$ vanishes and so $m_{g} * P_{f}=u t^{l}+$ h.o. $(t)$ where $0 \neq u \in \mathbb{Z}_{2}\langle\operatorname{Crit}(f)\rangle$ and $l>0$. As $\phi\left(m_{g} * P_{f}\right)=z_{i_{0}} t^{i_{0}}+$ h.o. $(t)$ it follows that $l \leq i_{0}$. By the definition of the moduli spaces giving the module action (in Section 3.2), this implies the claim at point (i) of our theorem: for a generic $J$ there exists a nonconstant $J$-disk $v:(D, \partial D) \rightarrow(M, L)$ with $v(0)=m_{g}$ and such that $\mu([v]) \leq l N_{L} \leq i_{0} N_{L} \leq$ $\left(k / N_{L}-1\right) N_{L}=\left(k-N_{L}\right)$. In particular

$$
w(M \backslash L) \leq i_{0} N_{L} \eta \leq\left(k-N_{L}\right) \eta .
$$

This completes the proof of point (i) of our theorem.

We now turn to the proof of the point (ii) of the theorem. Recall that $S_{1}=\sum_{i=i_{0}}^{k / N_{L}-1} z_{i} t^{i}$ and that $1 \leq i_{0} \leq k / N_{L}-1$. By assumption $L$ is wide so $\delta^{+}=0$, hence by (32) we get $a^{\prime} * S_{1}=[L] t^{k / N_{L}}$. Expanding this equality gives

$$
\sum_{i_{0} \leq r+i \leq k / N_{L}} a_{r}^{\prime} * z_{i} t^{r+i}=[L] t^{k / N_{L}},
$$

where we have written $a^{\prime}=\sum_{r \geq 0} a_{r}^{\prime} t^{r}$ (with $a_{r}^{\prime} \in \mathbb{Z}_{2}\langle$ Crit $(g)\rangle$ ). The key remark is that

$$
\exists r \geq 0, i \geq i_{0} \geq 1, \quad \text { such that }\left(a_{r}^{\prime} * z_{i}\right) t^{r+i}=[L] t^{k / N_{L}}+\text { 1.o. }(t) .
$$

Thus $\phi\left(a_{r}^{\prime} * \psi\left(z_{i}\right)\right) t^{r+i}=[L] t^{k / N_{L}}+$ l.o. $(t)$. Write $\psi\left(z_{i}\right)=\sum_{q \geq 0} x_{q} t^{q}$, where $x_{q} \in \mathbb{Z}_{2}\langle\operatorname{Crit}(f)\rangle$. It follows that there exists $q$ such that $\phi\left(a_{r}^{\prime} * x_{q}\right) t^{q+r+i}=$ $[L] t^{k / N_{L}}+$ l.o. $(t)$. Finally, writing $a_{r}^{\prime} * x_{q}=\sum_{s \geq 0} p_{s} t^{s}$ we deduce that there exists $s$ such that

$$
\phi\left(p_{s}\right) t^{s+q+r+i}=[L] t^{k / N_{L}}+\text { 1.o. }(t) .
$$

Put $\tau=k / N_{L}-(s+q+r+i)$. There are two main cases to be considered: $s+q+r+i<$ $k / N_{L}$ (ie $\tau>0$ ) and $s+q+r+i=k / N_{L}$ (ie $\tau=0$ ). Before considering each case it is important to note that as $i \geq i_{0} \geq 1$ we always have $s, q, r<k / N_{L}$.

Case $1(\tau>0)$ We have $\phi\left(p_{s}\right)=t^{\tau}[L]+$ 1.o. $(t)$ with $\tau>0$ and we deduce from Lemma 5.1.3 that there exists a critical point $w$ such that $d w=P_{f} t^{\tau^{\prime}}+$ 1.o.( $(t)$ with $0<\tau^{\prime} \leq \tau$. It follows that there exists a nonconstant $J$-disk through $P_{f}$ with Maslov 
index $\leq \tau^{\prime} N_{L} \leq \tau N_{L}<k$, which proves the desired uniruling property of $L$. In view of (33) we also have

$w(L)+2 w(M \backslash L) \leq 2 \tau N_{L} \eta+2 i_{0} N_{L} \eta=2\left(\frac{k}{N_{L}}-s-q-r-i+i_{0}\right) N_{L} \eta \leq 2 k \eta$.

Case $2(\tau=0)$ This means that $\phi\left(p_{s}\right)=[L]$, hence $p_{s}=P_{f}$. Therefore

$$
a_{r}^{\prime} * x_{q}=P_{f} t^{s}+\text { 1.o. }(t) \text {. }
$$

There are again two cases: $s>0$ and $s=0$.

Case 2-i $(\tau=0, s>0)$ We obtain from (35) that there exists a nonconstant $J$-disk through $P_{f}$ with Maslov index $\leq s N_{L}<k$. As in case 1 above we also have

$$
w(L)+2 w(M \backslash L) \leq 2 s N_{L} \eta+2 i_{0} N_{L} \leq 2(s+i) N_{L} \eta \leq 2 k \eta .
$$

Case 2-ii $(\tau=0, s=0)$ We will show now that this case is impossible. To see this, first note that by (35) we have that $a_{r}^{\prime} * x_{q}=P_{f}$, hence $a_{r}^{\prime}=P_{g}, x_{q}=P_{f}$. This implies that $a=[M] t^{r}+$ 1.o. $(t)$. Write $a=[M] t^{r}+a_{r-1} t^{r-1}+\cdots+a_{1} t+a_{0}$, where $a_{j} \in H_{*}\left(M ; \mathbb{Z}_{2}\right)$ are homogeneous elements. Recall that $[\mathrm{pt}] * a=[M] t^{k / N_{L}}$. Therefore

$$
[M] t^{k / N_{L}}=[\mathrm{pt}] * a=[\mathrm{pt}] t^{r}+[\mathrm{pt}] * a_{r-1} t^{r-1}+\cdots+[\mathrm{pt}] * a_{1} t+[\mathrm{pt}] * a_{0} .
$$

It follows that there exists $0 \leq j \leq r-1$ such that $\left([\mathrm{pt}] * a_{j}\right) t^{j}=[\mathrm{pt}] t^{r}+$ h.o. $(t)$, hence $\left([\mathrm{pt}] * a_{j}\right)=[\mathrm{pt}] t^{r-j}+$ h.o. $(t)$. Clearly this equality takes place in the image of the inclusion $Q H\left(M ; \Gamma^{+}\right) \rightarrow Q H\left(M ; \Lambda^{+}\right)$defined by $s \rightarrow t^{2 C_{M} / N_{L}}$, therefore we actually have in $Q H\left(M ; \Gamma^{+}\right)$

$$
\left([\mathrm{pt}] * a_{j}\right)=[\mathrm{pt}] s^{(r-j) N_{L} / 2 C_{M}}+\text { h.o. }(s) .
$$

Note also that by the definition of $a_{j}$ we have $a_{j} \neq[M]$. We will now show that such a relation is impossible in quantum homology. To see this note that $r-j>0$ since $r-j=0$ would give [pt] $* a_{j}=[\mathrm{pt}]$ which is possible only if $a_{j}=[M]$ which is not the case. As $r-j>0$, the relation (36) implies that there exists a homology class $A \in H_{2}^{S}(M)$ with $2 c_{1}(A)=(r-j) N_{L}$ such that $\mathrm{GW}\left([\mathrm{pt}], a_{j},[M] ; A\right) \neq 0$. In particular, for generic $J$, the moduli space of (simple) $J$-holomorphic rational curves $u: \mathbb{C} P^{1} \rightarrow M$ in the class $A$ which pass through a given point in $M$ and intersect a cycle representing $a_{j}$ is not empty. To estimate the dimension of this space denote by $\mathcal{M}(A, J)$ the space of simple rational curves in the class $A$ and by $G=\operatorname{Aut}\left(\mathbb{C} P^{1}\right) \approx P S L(2, \mathbb{C})$ the group of biholomorphisms of $\mathbb{C} P^{1}$. Consider the evaluation map

$$
\text { ev: }\left(\mathcal{M}(A, J) \times \mathbb{C} P^{1} \times \mathbb{C} P^{1}\right) / G \rightarrow M \times M, \quad \operatorname{ev}\left(u, z_{1}, z_{2}\right)=\left(u\left(z_{1}\right), u\left(z_{2}\right)\right) .
$$


The moduli space in question is $\mathrm{ev}^{-1}\left(\mathrm{pt} \times W_{a_{j}^{\prime}}^{u}\right)$, where we recall that $a_{j}^{\prime} \in \mathbb{Z}_{2}\langle\operatorname{Crit}(g)\rangle$ is a Morse cycle representing $a_{j}$ and $W_{a_{j}^{\prime}}^{u}$ stands for the unstable submanifolds associated to the critical points in $a_{j}^{\prime}$. By transversality we obtain the following dimension formula:

$\operatorname{dimev}{ }^{-1}\left(\operatorname{pt} \times W_{a_{j}^{\prime}}^{u}\right)=2 n+2 c_{1}(A)+2+2-6+\left|a_{j}\right|-4 n=-2 n+(r-j) N_{L}-2+\left|a_{j}\right|$.

On the other hand, $\left|a_{j}\right|=2 n-(r-j) N_{L}$ by (36). Putting this into the dimension formula we get $\operatorname{dim~ev}^{-1}\left(\mathrm{pt} \times W_{a_{j}^{\prime}}^{u}\right)=-2$, contradicting the fact that this space is not empty. This rules out Case 2-ii and concludes the proof of Theorem 1.2.3.

\subsection{Proof of Theorem 1.2.7}

We first recall the definition of the spectral invariants as well as some other basic facts and we fix some conventions.

Consider a generic pair $(H, J)$ consisting of a 1-periodic Hamiltonian $H: M \times S^{1} \rightarrow$ $\mathbb{R}$ and an almost complex structure $J$ so that the Floer complex $C F_{*}(H, J)$ is well defined. (Here, $C F(H, J)$ is the Floer complex for periodic orbits Floer homology.) Let $I=\{\bar{\gamma}=(\gamma, \hat{\gamma})\} / \sim$ where $x$ is a contractible 1-periodic orbit of the Hamiltonian flow of $H, \hat{\gamma}: D \rightarrow M$ is a disk-capping of $\gamma$ (ie $\left.\hat{\gamma}\right|_{\partial D}=\gamma$ ) and the equivalence relation $\sim$ is $\bar{\gamma} \sim \bar{\gamma}^{\prime}$ if $\gamma=\gamma^{\prime}$ and $\omega(\hat{\gamma})=\omega\left(\hat{\gamma}^{\prime}\right)$. Notice that $I$ is a $\Gamma$-module (we recall that $\Gamma=\mathbb{Z}_{2}\left[s^{-1}, s\right]$ ), the elements of $\Gamma$ acting by changing the capping: $s \cdot(\gamma, \hat{\gamma})=\left(\gamma, \hat{\gamma}_{1}\right)$, where $\omega\left(\hat{\gamma}_{1}\right)=\omega(\hat{\gamma})-2 C_{M} \eta$. As $\Lambda$ is a $\Gamma$-module we will define the Floer complex of interest here as: $C F(H, J ; \Lambda)=\mathbb{Z}_{2}\langle I\rangle \otimes_{\Gamma} \Lambda$ endowed with the usual Floer differential.

Fix also a Morse function $f: L \rightarrow \mathbb{R}$ as well as a Riemannian metric $\rho$ on $L$ so that the pearl complex $\mathcal{C}^{+}(L ; f, \rho, J)$ is well defined.

We need to provide a Floer-theoretic description of our module operation $\circledast$ which involves the two complexes above. This is based on moduli spaces $\mathcal{P}_{\mathcal{T}}^{\prime}$ similar to the ones used in Section 3.2 (c) except that the vertex of valence three in the string of pearls is now replaced by a half-tube with boundary on $L$ and with the $-\infty$ end on an element $\bar{\gamma} \in I$. The symbol of the tree is $(\bar{\gamma}, x: y)$. The total homotopy class $\lambda$ of the configuration obtained in this way is computed by using the capping associated to $\gamma$ to close the semi-tube to a disk and adding up the homotopy class of this disk to the homotopy classes of the other disks in the string of pearls. More explicitly, a half tube as before is a solution

$$
u:(-\infty, 0] \times S^{1} \rightarrow M
$$

of Floer's equation

$$
\partial u / \partial s+J \partial u / \partial t+\nabla H(u, t)=0
$$


with the boundary conditions

$$
u\left(\{0\} \times S^{1}\right) \subset L \lim _{s \rightarrow-\infty} u(s, t)=\gamma(t) .
$$

The marked points on the "exceptional" vertex which corresponds to $u$ are so that the point $u(0,1)$ is an exit point for a flow line and $u(0,-1)$ is the entry point. See Figure 6. Both compactification and bubbling analysis for these moduli spaces are similar to what has been discussed before to which is added the study of transversality and bubbling for the spaces of half-tubes as described by Albers in [2]. As described in [2], an additional assumption is needed for this part: $H$ is assumed to be such that no periodic orbit of $X^{H}$ is completely included in $L$.

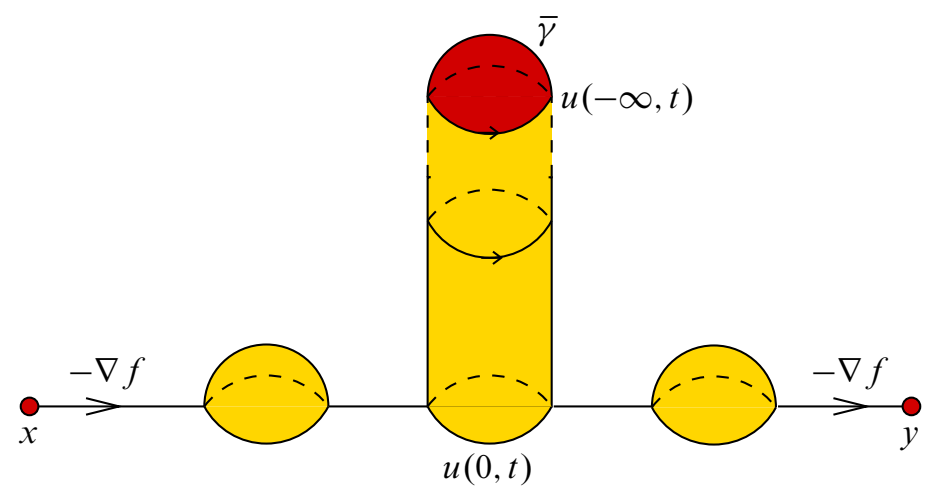

Figure 6: An element $v \in \mathcal{P}_{\mathcal{T}}^{\prime}$

Counting elements in these moduli spaces defines an operation:

$\circledast_{F}: C F(H, J ; \Lambda) \otimes_{\Lambda} \mathcal{C}(L ; f, \rho, J) \rightarrow \mathcal{C}(L ; f, \rho, J)$.

Fix a Morse-Smale pair $\left(g, \rho_{M}\right)$ on $M$ and let $C^{+}(g)$ be the corresponding Morse complex tensored with $\Lambda^{+}$. Recall the module action defined in Section 3.2 (c):

$$
\circledast: C^{+}(g) \otimes_{\Lambda^{+}} \mathcal{C}^{+}(L ; f, \rho, J) \rightarrow \mathcal{C}^{+}(L ; f, \rho, J) .
$$

There are maps induced by the inclusion $\Lambda^{+} \rightarrow \Lambda$

$$
C^{+}(g) \rightarrow C^{+}(g) \otimes_{\Lambda^{+}} \Lambda=C(g) \quad \text { and } \quad \mathcal{C}^{+}(L ; f, \rho, J) \rightarrow \mathcal{C}(L ; f, \rho, J)
$$

which we will denote in both cases by $p$.

We will now use the Hamiltonian version of the Piunikin-Salamon-Schwarz homomorphism [48]: $\widetilde{\mathrm{PSS}}: C(g) \rightarrow C F(H, J ; \Lambda)$. Standard arguments show that there is a 
chain homotopy $\xi: C^{+}(g) \otimes_{\Lambda^{+}} \mathcal{C}^{+}(L ; f, \rho, J) \rightarrow \mathcal{C}(L ; f, \rho, J)$ which satisfies

$$
\widetilde{\mathrm{PSS}}(p(x)) \circledast_{F} p(y)-p(x \circledast y)=d \xi(x \otimes y)-\xi(d(x \otimes y)),
$$

for every $x \in C^{+}(g), y \in \mathcal{C}^{+}(L ; f, \rho, J)$.

The Floer complex $C F_{*}(H, J)$ is filtered by the values of the action functional

$$
\mathcal{A}_{H}(\bar{\gamma})=\int_{S^{1}} H(\gamma(t), t) d t-\int_{D} \hat{\gamma}^{*} \omega
$$

where $\bar{\gamma}=(\gamma, \hat{\gamma})$, with $\gamma$ a contractible $C^{\infty}$-loop in $M$ and $\hat{\gamma}$ a cap of this loop. This action is compatible with the action of $\Gamma$ and we extend it on the generators of $C F(H, J ; \Lambda)=\mathbb{Z}_{2}\langle I\rangle \otimes_{\Gamma} \Lambda$ by: $\mathcal{A}_{H}\left(\bar{\gamma} \otimes t^{k}\right)=\mathcal{A}_{H}(\bar{\gamma})-k \eta N_{L}$ (where $\eta$ is the monotonicity constant). The filtration of order $v \in \mathbb{R}$ of the Floer complex, $C F^{\leq v}$, is the graded $\mathbb{Z}_{2}$-vector space generated by all the elements $\bar{x} \otimes \lambda$ of action at most $\nu$.

We emphasize that all the homology and cohomology classes to be considered below are homogeneous. We now recall the definition of spectral invariants following Schwarz [49] and Oh [47]. Fix $\alpha \in Q H_{*}(M ; \Lambda)=\left(H\left(M ; \mathbb{Z}_{2}\right) \otimes \Lambda\right)_{*}$ and define the spectral invariant $\sigma(\alpha, H)$ of $\alpha$ by

$$
\sigma(\alpha, H)=\inf \left\{v: \operatorname{PSS}(\alpha) \in \operatorname{Image}\left(H\left(C F^{\leq v}\right) \rightarrow H F(H, J ; \Lambda)\right)\right\},
$$

where PSS: $Q H_{*}(M ; \Lambda) \rightarrow H F_{*}(H, J ; \Lambda)$ is the morphism induced in homology by $\widetilde{\text { PSS }}$. Notice that by convention we have $\sigma(0 ; H)=-\infty$. Assuming that $H$ is normalized, it is well known that $\sigma(\alpha, H)$ depends only on the class $\left[\phi^{H}\right] \in \widetilde{\operatorname{Ham}}(M)$ and on $\alpha$, and is therefore denoted by $\sigma\left(\alpha, \phi^{H}\right)$. We refer the reader to Oh [47; 45; 46; 41], Schwarz [49] and McDuff and Salamon [40] for the foundations of the theory of spectral invariants. See also Viterbo [53] for an earlier approach to the subject.

Let $L \subset M$ be a monotone Lagrangian submanifold. Theorem 1.2.7 is an immediate consequence of the first part of Lemma 5.3.1 below. To state it we fix some more notation. As discussed before, the inclusion $\Lambda^{+} \rightarrow \Lambda$ induces a map $p: \mathcal{C}^{+}(L ; f, \rho, J) \rightarrow \mathcal{C}(L ; f, \rho, J)$ which is canonical in homology. We continue to denote the induced map in homology by $p$ too. Denote by $I Q^{+}(L)$ the image of $p: Q^{+} H(L) \rightarrow Q H(L)$ and notice that $I Q^{+}(L)$ is a $\Lambda^{+}$module so that it makes sense to say whether a class $z \in I Q^{+}(L)$ is divisible by $t$ in $I Q^{+}(L)$ : this means that there is some $z^{\prime} \in I Q^{+}(L)$ so that $z=t z^{\prime}$.

Lemma 5.3.1 $\quad$ (i) Assume that $\alpha \in Q H_{*}\left(M ; \Lambda^{+}\right), x, y \in Q^{+} H_{*}(L)$ are so that $p(y)$ is not divisible by $t$ in $I^{+}(L)$ and $\alpha * x=y t^{s}$. Then we have the following inequality for every $\phi \in \widetilde{\operatorname{Ham}}(M)$ :

$$
\sigma(\alpha ; \phi) \geq \operatorname{depth}_{L}(\phi)-s N_{L} \eta .
$$


(ii) Let $x \in Q^{+} H_{*}(L)$ and let $\phi \in \widetilde{\operatorname{Ham}}(M)$. Then

$$
\sigma\left(i_{L}(x) ; \phi\right) \leq \text { height }_{L}(\phi)
$$

where $i_{L}: Q H(L) \rightarrow Q H(M ; \Lambda)$ is the quantum inclusion from Theorem A (iii).

The second point of the lemma is an extension of a result of Albers [2].

Before proving Lemma 5.3.1, we show how it implies Theorem 1.2.7. Indeed, if $L \subset M$ is not narrow, then $[L] \in Q H(L)$ is not trivial and we have $[M] *[L]=[L]$ which implies the first point of Theorem 1.2.7 because, for degree reasons, $p([L])$ is not divisible by $t$ in $I Q^{+}(L)$. Moreover, if $M$ is point invertible of order $k$, then there is $a \in Q H\left(M ; \Lambda^{+}\right)$so that $[\mathrm{pt}] * a=[M] t^{k / N_{L}}$. Therefore, setting $a^{\prime}=a *[L] \in Q^{+} H(L)$ we get $[\mathrm{pt}] * a^{\prime}=[L] t^{k / N_{L}}$ and by applying the lemma for $\alpha=[\mathrm{pt}], x=a^{\prime}, y=[L]$ we deduce Theorem 1.2.7 (ii).

Proof of Lemma 5.3.1 (i) We fix $\phi \in \widetilde{\operatorname{Ham}}(M)$. By inspecting the definition of depth in Section 1.2.3 we see that the inequality we need to prove is reduced to showing that for every normalized Hamiltonian $H$ with $[H]=\phi$ there exists a loop $\gamma: S^{1} \rightarrow L$ such that

$$
\sigma(\alpha, \phi)-\int_{S^{1}} H(\gamma(t), t) d t+s \eta N_{L} \geq 0
$$

By a small perturbation of $H$ we may assume that no closed orbit of $H$ is contained in $L$.

Given any $\epsilon>0$, in view of the definition of $\sigma(\alpha, H)$, we may find in $C F^{\leq \sigma(\alpha, H)+\epsilon}$ a cycle $\zeta$ with $[\zeta]=\operatorname{PSS}(\alpha) \in H F(H, J ; \Lambda)$. Write $\zeta=\sum \overline{\gamma_{i}} \otimes t^{k_{i}}$ where $\overline{\gamma_{i}}$ are generators of $C F(H, J)$ and $t^{k_{i}} \in \Lambda, k_{i} \in \mathbb{Z}$. Represent also $x$ as a cycle in $\mathcal{C}^{+}(L ; f, \rho, J)$, $x=\left[x^{\prime}\right]$ with $x^{\prime}=\sum_{i \geq 0} x_{i} t^{i}, x_{i} \in \mathbb{Z}_{2}\langle\operatorname{Crit}(f)\rangle$. Similarly, represent also $y$ by a cycle $y^{\prime}$ in $\mathcal{C}^{+}(L ; f, \rho, J)$. From Equation (38) we deduce that $\zeta \circledast_{F} x^{\prime}-y^{\prime} t^{s} \in \operatorname{Im}(d)$, where $d$ is the differential in $\mathcal{C}(L ; f, \rho, J)$. Write $\zeta \circledast_{F} x^{\prime}=\sum_{i} z_{i} t^{i}$ with $i \in \mathbb{Z}$, $z_{i} \in \mathbb{Z}_{2}\langle\operatorname{Crit}(f)\rangle$ (note that here we cannot assume anymore that $i \geq 0$ only). The fact that $y^{\prime}$ is not divisible by $t$ in $I Q^{+}(L)$ implies that there is some $z_{r} \neq 0$ with $r \leq s$. But this means that there are $\gamma_{i}$ and $x_{j}$ so that $\left(\overline{\gamma_{i}} \otimes t^{k_{i}}\right) \circledast_{F} x_{j} t^{j}=z_{r} t^{r}+\cdots$ (where $\cdots$ stands for other terms). This means that there are critical points $x_{j}^{0}, z_{r}^{0} \in \operatorname{Crit}(f)$ (participating in $x_{j}$ and $z_{r}$ ) so that the moduli space $\mathcal{P}_{\mathcal{T}}^{\prime}$ (described at the beginning of the section), of symbol $\left(\bar{\gamma}_{i}, x_{j}^{0}: z_{r}^{0}\right)$ and with $\mu(\mathcal{T})=\left(r-j-k_{i}\right) N_{L}$, is not void. We now consider an element $v \in \mathcal{P}_{\mathcal{T}}^{\prime}$ and we focus on the corresponding half-tube $u$ 
(which is part of $v$ ). The usual energy estimate for this half-tube gives

$$
\begin{aligned}
0 & \leq \int_{-\infty}^{0} \int_{S^{1}}\|\partial u / \partial s\|^{2} d t d s \\
& =\int_{(-\infty, 0] \times S^{1}} u^{*} \omega+\int_{S^{1}} H\left(\gamma_{i}(t), t\right) d t-\int_{S^{1}} H(u(0, t), t) d t,
\end{aligned}
$$

hence: $\quad \int_{S^{1}} H(u(0, t), t) d t \leq \int_{(-\infty, 0] \times S^{1}} u^{*} \omega+\int_{S^{1}} H\left(\gamma_{i}(t), t\right) d t$.

We now claim that:

$$
\mathcal{A}_{H}\left(\gamma_{i}\right)+\left(r-j-k_{i}\right) \eta N_{L} \geq \int_{(-\infty, 0] \times S^{1}} u^{*} \omega+\int_{S^{1}} H\left(\gamma_{i}(t), t\right) d t .
$$

Indeed, $\eta \mu(\mathcal{T})$ equals the symplectic area of all the disks in $v+$ the area of the tube $u+$ the area of the cap $\widehat{\gamma}_{i}$ corresponding to $\overline{\gamma_{i}}$. The inequality (41) now follows because the disks in $v$ are $J$-holomorphic hence their area is nonnegative. But now $\sigma(\alpha, H)+\epsilon \geq \mathcal{A}_{H}\left(\overline{\gamma_{i}} \otimes t^{k_{i}}\right)=\mathcal{A}_{H}\left(\overline{\gamma_{i}}\right)-k_{i} \eta N_{L}$ and as $j \geq 0, s \geq r$ we obtain

$$
\sigma(\alpha, H)+\epsilon+s \eta N_{L} \geq \int_{(-\infty, 0] \times S^{1}} u^{*} \omega+\int_{S^{1}} H\left(\gamma_{i}(t), t\right) d t
$$

so that by taking $\gamma(t)=u(0, t)$ we deduce inequality (40).

(ii) Given a Hamiltonian $H$ with $\phi=\phi^{H}$, a Morse function $f$, a generic metric $\rho$ and a generic almost complex structure $J$ we will define a chain map

$$
\widetilde{i}_{L}: \mathcal{C}(L ; f, \rho, J) \rightarrow C F(H, J ; \Lambda)
$$

so that the maps induced in homology by $\widetilde{\mathrm{PSS}} \circ i_{L}$ and by $\tilde{i}_{L}$ are equal. To describe this map, fix a particular capping $\widehat{\gamma}^{\prime}$ for each contractible 1 -periodic orbit $\gamma$ of the Hamiltonian vector field $X^{H}$ of $H$. We denote these pairs by $\tilde{\gamma}=\left(\gamma, \hat{\gamma}^{\prime}\right)$.

For a critical point $p \in \operatorname{Crit}(f)$ we define

$$
\tilde{i}_{L}(p)=\sum_{\mathcal{T}, \gamma} \#_{2}\left(\mathcal{P}_{\mathcal{T}}^{\prime \prime}\right) \tilde{\gamma} \otimes t^{\mu(\mathcal{T}) / N_{L}}
$$

where the moduli spaces $\mathcal{P}_{\mathcal{T}}^{\prime \prime}$ are similar to the ones used in Section 3.2 (d) except that the last (exceptional) vertex there as well as its exiting edge are replaced here by a Floer semi-tube; the Maslov index $\mu(\mathcal{T})$ is the sum of the Maslov indices of the disks in the chain of pearls summed with the Maslov index of the tube glued to the disk $\hat{\gamma}^{\prime}$ with reversed orientation. More precisely, the moduli spaces $\mathcal{P}_{\mathcal{T}}^{\prime \prime}$ used here correspond 
to trees $\mathcal{T}$ of symbol $(p: \tilde{\gamma})$. An element $v \in \mathcal{P}_{\mathcal{T}}^{\prime \prime}$ consists of a pair $\left(u^{\prime}, u^{\prime \prime}\right)$ where $u^{\prime \prime}$ is a Floer semi-tube

$$
u^{\prime \prime}:[0, \infty) \times S^{1} \rightarrow M
$$

satisfying Floer's Equation (37) with the boundary conditions

$$
u^{\prime \prime}\left(\{0\} \times S^{1}\right) \subset L, \quad \lim _{s \rightarrow \infty} u^{\prime \prime}(s, t)=\gamma(t)
$$

and $u^{\prime}$ is a string of pearls $u^{\prime}=\left(u_{1}, \ldots, u_{k}\right)$ in $M$ associated to $f$, starting at the critical point $p \in \operatorname{Crit}(f)$ and so that the last incidence condition is

$$
\exists t>0, \gamma_{t}^{f}\left(u_{k}(1)\right)=u^{\prime \prime}(0,-1) .
$$

In other words, $u^{\prime}$ is an element as of a moduli space as those considered in the construction of the pearl differential Section 3.2 (a) except that the endpoint is not $\in \operatorname{Crit}(f)$ but $u^{\prime \prime}(0,-1)$. The Maslov index is given by $\mu(\mathcal{T})=\mu\left(u^{\prime}\right)+\mu\left(u^{\prime \prime} \#\left(\hat{\gamma}^{\prime}\right)^{-1}\right)$ where $\left(\widehat{\gamma}^{\prime}\right)^{-1}$ is the disk with the opposed orientation compared to $\hat{\gamma}^{\prime}$, and $u^{\prime \prime} \#\left(\widehat{\gamma}^{\prime}\right)^{-1}$ indicates the surface obtained by gluing the tube $u^{\prime \prime}$ and the capping disk $\left(\widehat{\gamma}^{\prime}\right)^{-1}$ along $\gamma$. The sum in (42) is taken over all $(\mathcal{T}, \gamma)$ such that $|p|-\mu(\tilde{\gamma})+\mu(\mathcal{T})=0$. It is easy to see that the definition of $\tilde{i}_{L}$ does not depend on the specific choice of the cappings $\hat{\gamma}^{\prime}$ associated to each $\gamma$.

The regularity issues for the moduli spaces $\mathcal{P}_{\mathcal{T}}^{\prime \prime}$ are similar to those discussed before. Finally, standard arguments show that by extending this definition by linearity over $\Lambda$ we obtain a chain map and that, the map induced in homology by $\widetilde{i}_{L}$ coincides with PSS $\circ i_{L}$.

The next step is to establish an action estimate for the configurations $v=\left(u^{\prime}, u^{\prime \prime}\right) \in \mathcal{P}_{\mathcal{T}}^{\prime \prime}$ considered above. We recall that if $\tilde{\gamma}$ is a capped orbit as above, the element $\tilde{\gamma} \otimes t^{k}$ is a generator of $C F(H, J ; \Lambda)$ and its action is $\mathcal{A}_{H}(\tilde{\gamma})-k \eta N_{L}$. The energy estimate associated to $u^{\prime \prime}$ gives

$$
0 \leq \int_{S^{1}} H\left(u^{\prime \prime}(0, t), t\right) d t-\int_{S_{1}} H(\gamma(t), t) d t+\int_{[0, \infty) \times S^{1}}\left(u^{\prime \prime}\right)^{*} \omega,
$$

and so

$$
\mathcal{A}_{H}(\tilde{\gamma})=-\int_{D}\left(\hat{\gamma}^{\prime}\right)^{*} \omega+\int_{S^{1}} H(\gamma(t), t) d t \leq \int_{S^{1}} H\left(u^{\prime \prime}(0, t), t\right) d t+\omega\left(\left[u^{\prime \prime} \#\left(\hat{\gamma}^{\prime}\right)^{-1}\right]\right) \text {. }
$$

Clearly, $\omega\left(\left[u^{\prime \prime} \#\left(\hat{\gamma}^{\prime}\right)^{-1}\right]\right)=\mu(\mathcal{T}) \eta-\omega\left(\left[u^{\prime}\right]\right)$ and as $\omega\left(\left[u^{\prime}\right]\right) \geq 0$ we deduce

$$
\mathcal{A}_{H}\left(\tilde{\gamma} \otimes t^{\mu(\mathcal{T}) / N_{L}}\right) \leq \text { height }_{L}(\phi) .
$$

Let $x \in Q^{+} H(L)$ and $x^{\prime}=\sum_{i \geq 0} x_{i} t^{i} \in \mathcal{C}^{+}(L ; \underset{\sim}{f}, \rho, J), x_{i} \in \mathbb{Z}_{2}\langle\operatorname{Crit}(f)\rangle$ be a pearl cycle that represents $x$. Denote $\sum_{i} \tilde{\gamma}_{i} \otimes t^{k_{i}}$ by $\widetilde{i}_{L}\left(x^{\prime}\right)$. Consider any of the terms in 
this sum, say $\tilde{\gamma}_{j} \otimes t^{k_{j}}$. There exists $r \geq 0$ and a critical point $x_{r}^{0}$ participating in $x_{r}$, so that $\widetilde{i}_{L}\left(x_{r}^{0} t^{r}\right)$ contains $\tilde{\gamma}_{j} \otimes t^{k_{j}}$. As $\widetilde{i}_{L}$ is $\Lambda$-linear this means that $\widetilde{i}_{L}\left(x_{r}^{0}\right)$ contains $\tilde{\gamma}_{j} \otimes t^{k_{j}-r}$. From (43) we now obtain

$$
\mathcal{A}\left(\tilde{\gamma}_{j} t^{k_{j}}\right)=\mathcal{A}\left(\tilde{\gamma}_{j} t^{\left(k_{j}-r\right)}\right)-r N_{L} \eta \leq \operatorname{height}_{L}(\phi)-r N_{L} \eta \leq \operatorname{height}_{L}(\phi) .
$$

Finally, since $\tilde{i}_{L}$ and PSS $\circ i_{L}$ coincide in homology we can represent $\operatorname{PSS}\left(i_{L}(x)\right)$ as a linear combination of generators of $C F(H, J ; \Lambda)$ each of action at most height ${ }_{L}(\phi)$ which implies our claim.

Remark 5.3.2 (a) Sometimes the point (ii) of Lemma 5.3.1 can be used to estimate from above spectral invariants of homology classes $\alpha \in H_{*}(M)$. For example, it is easy to see that $i_{L}([L])=\operatorname{inc}([L])$, where $\operatorname{inc}_{*}: H_{*}\left(L ; \mathbb{Z}_{2}\right) \rightarrow H_{*}\left(M ; \mathbb{Z}_{2}\right)$ is the map induced by the inclusion $L \subset M$. Therefore whenever inc $*([L]) \neq 0$ we obtain $\sigma\left(\operatorname{inc}_{*}([L]), \phi\right) \leq \operatorname{height}_{L}(\phi)$ for any $\phi \in \widetilde{\operatorname{Ham}}(M)$.

(b) In a point invertible manifold the first part of Lemma 5.3.1 provides an estimate from below of $\sigma([\mathrm{pt}], \phi)$ and so, in view of the proofs of the intersection results discussed in Corollaries 2.4.1 and 1.2.8, it is particularly important to get also an estimate from the above. The natural idea is to write $[\mathrm{pt}]=i_{L}(x)$ for some class $x$. However, there are cases when [pt] is not in the image of this map $i_{L}$ - see for example the case of the quadric $Q^{2 n}$ described in Section 6.3.3.

(c) In case $\Lambda=\Gamma$ we have $C F(H, J ; \Lambda)=C F(H, J)=\mathbb{Z}_{2}\langle I\rangle$, where $I$ is the set of contractible 1-periodic orbits of $X^{H}$ together with all possible cappings (modulo the usual identifications) $I=\{\bar{\gamma}=(\gamma, \hat{\gamma})\} / \sim$. In this case the map $\tilde{i}_{L}$ can be written as $\widetilde{i}_{L}(p)=\sum_{\mathcal{T}, \bar{\gamma}} \#_{2}\left(\mathcal{P}_{\mathcal{T}}^{\prime \prime \prime}\right) \bar{\gamma}$ where the moduli space $\mathcal{P}_{\mathcal{T}}^{\prime \prime \prime}$ contains configurations as those in $\mathcal{P}_{\mathcal{T}}^{\prime \prime}$ but with the additional condition that $\mu(\mathcal{T})=0$. Indeed, as $\Lambda=\Gamma$ any element $\tilde{\gamma} \otimes t^{k}$ can be written uniquely as some $\bar{\gamma}$.

If additionally, we have $N_{L}>n+1$, then a dimension count shows that for the configurations $v=\left(u^{\prime}, u^{\prime \prime}\right)$ used to define $\widetilde{i}_{L}$ we have $\mu\left(u^{\prime}\right)=0$ and so there are no $J$-disks present in the definition of $\widetilde{i}_{L}$. Under these assumptions $\widetilde{i}_{L}$ coincides with a map introduced by Albers in [2].

(d) It is possible to define a pseudo-valuation $v: Q H(L) \rightarrow \mathbb{Z} \cup\{\infty\}$ as follows. Notice first that for any $a \in Q H(L)$ there exists $k \in \mathbb{Z}$ so that $t^{k} a \in I Q^{+}(L)$. Define

$$
v(a)=\max \left\{s \in \mathbb{Z} \mid t^{-s} a \in I Q^{+}(L)\right\} \in \mathbb{Z} \cup\{\infty\} .
$$

It is easy to see that $v$ is well defined, and that it satisfies $v(a) \geq 0$ if and only if $a \in I Q^{+}(L), v(a)=\infty$ if and only if $a=0, v(a+b) \geq \min \{v(a), v(b)\}, v(a * b) \geq$ $v(a)+v(b)$, and $v(t a)=v(a)+1$. A similar function to $v$ has already been considered 
by Entov and Polterovich [30] in the context of ambient quantum homology. The inequality at point (i) of Lemma 5.3.1 can now be reformulated as:

$$
\sigma(\alpha, \phi)-\operatorname{depth}_{L}(\phi) \geq(v(x)-v(\alpha * x)) N_{L} \eta, \quad \forall \alpha \in Q H(M ; \Lambda), x \in Q H(L) .
$$

\subsection{Proof of Theorem 2.4.1}

Recall the setting of this theorem. Given $L_{0}, L_{1} \subset M$, monotone Lagrangian submanifolds we have the two associated rings $\Lambda_{0}=\mathbb{Z}_{2}\left[t_{0}^{-1}, t_{0}\right], \Lambda_{1}=\mathbb{Z}_{2}\left[t_{1}^{-1}, t_{1}\right]$ graded by $\operatorname{deg} t_{0}=-N_{L_{0}}$ and $\operatorname{deg} t_{1}=N_{L_{1}}$ as well as the ring $\Lambda_{0,1}=\Lambda_{0} \otimes_{\Gamma} \Lambda_{1}$ where $\Gamma=\mathbb{Z}_{2}\left[s^{-1}, s\right],|s|=-2 C_{\min }$. Recall also the two canonical maps: the quantum inclusion $i_{L_{0}}: Q H_{*}\left(L_{0} ; \Lambda_{0,1}\right) \rightarrow Q H_{*}\left(M ; \Lambda_{0,1}\right)$ and $j_{L_{1}}: Q H_{*}\left(M ; \Lambda_{0,1}\right) \rightarrow$ $Q H_{*-n}\left(L_{1} ; \Lambda_{0,1}\right)$, defined by $j_{L_{1}}(a)=a *\left[L_{1}\right]$. The claim of the theorem is that if the composition

$$
j_{L_{1}} \circ i_{L_{0}}: Q H_{*}\left(L_{0} ; \Lambda_{0,1}\right) \longrightarrow Q H_{*-n}\left(L_{1} ; \Lambda_{0,1}\right) .
$$

does not vanish, then $L_{0}$ and $L_{1}$ intersect.

We start the proof with a little more preparation. First note that since $\Lambda_{0,1}$ is a $\Gamma-$ module we can naturally extend the definition of periodic orbit Floer homology to coefficients in $\Lambda_{0,1}$ as the homology of the complex $C F\left(H, J ; \Lambda_{0,1}\right)=C F(H, J) \otimes_{\Gamma} \Lambda_{0,1}$. We denote this homology by $H F\left(H, J ; \Lambda_{0,1}\right)$. Moreover, the PSS isomorphism naturally extends to this case and we get an isomorphism PSS: $H F_{*}\left(H, J ; \Lambda_{0,1}\right) \rightarrow$ $Q H_{*}\left(M ; \Lambda_{0,1}\right)$. Similarly, we can extend the action functional to the generators of $C F\left(H, J ; \Lambda_{0,1}\right)$ by defining: $\mathcal{A}_{H}\left(\bar{x} \otimes t_{0}^{k_{0}} \otimes t_{1}^{k_{1}}\right)=\mathcal{A}_{H}(\bar{x})-k_{0} \eta_{0} N_{L_{0}}-k_{1} \eta_{1} N_{L_{1}}$. Here $\eta_{i}=\left.(\omega / \mu)\right|_{H_{2}^{D}\left(M, L_{i}\right)}, i=0,1$, are the monotonicity constants of the Lagrangians. (Clearly, $\eta_{0}=\eta_{1}$, unless $\left.\omega\right|_{\pi_{2}(M)}=0$ in which case we anyway have $C_{M}=\infty, \Gamma=\mathbb{Z}_{2}$ hence $C F\left(H, J ; \Lambda_{0,1}\right)=C F(H, J) \otimes \Lambda_{0} \otimes \Lambda_{1}$. $)$ It is easy to see that this extension of the action is well defined. With these conventions we have as before a filtration on $H F\left(H, J ; \Lambda_{0,1}\right)$ by action and we can define spectral numbers $\sigma_{\Lambda_{0,1}}(\alpha, \phi)$ for every $\alpha \in Q H\left(M ; \Lambda_{0,1}\right), \phi \in \widetilde{\operatorname{Ham}}(M)$, in a standard way. A straightforward algebraic argument shows that for classes $\alpha \in Q H(M) \subset Q H\left(M ; \Lambda_{0,1}\right)$ (as well as $\left.\alpha \in Q H\left(M ; \Lambda_{i}\right), i=0,1\right)$ these "new" spectral numbers coincide with the usual ones, ie $\sigma_{\Lambda_{0,1}}(\alpha, \phi)=\sigma(\alpha, \phi)$. (The point is that $\Lambda_{0,1}$ is a free module over $\Gamma$.) We will also need the ring $\Lambda_{0,1}^{+}=\Lambda_{0}^{+} \otimes_{\Gamma^{+}} \Lambda_{1}^{+}$. As before we have

$$
\Lambda_{0,1}^{+} \cong \mathbb{Z}_{2}\left[t_{0}, t_{1}\right] /\left\{t_{0}^{2 C_{M} / N_{L_{0}}}=t_{1}^{2 C_{M} / N_{L_{1}}}\right\}
$$

Next we remark that Lemma 5.3.1 continues to hold if we replace $L$ by one of the $L_{i}$ 's, say $L_{0}$, replace $\Lambda$ by $\Lambda_{0,1}, \Lambda^{+}$by $\Lambda_{0,1}^{+}$and the condition that " $p(y)$ is not divisible 
by $t$ in $I Q^{+}(L)$ " by " $p(y)$ is not divisible by $t_{0}$ in the image of the map $p$ " with $p$ the canonical "change of coefficients" map $p: Q H\left(L_{0} ; \Lambda_{0,1}^{+}\right) \rightarrow Q H\left(L_{0} ; \Lambda_{0,1}\right)$. The proof of the lemma carries out to this case without any essential modifications.

Since $j_{L_{1}} \circ i_{L_{0}} \neq 0$ there exists $x \in Q H\left(L ; \Lambda_{0,1}^{+}\right)$so that $j_{L_{1}} \circ i_{L_{0}}(x) \neq 0$. From the modified version of Lemma 5.3.1 discussed above, we deduce that for some constant $K$ depending only on $j_{L_{1}} \circ i_{L_{0}}(x)$ and for any $\phi \in \widetilde{\operatorname{Ham}}(M)$ we have

$$
\operatorname{depth}_{L_{1}}(\phi)-K \leq \sigma\left(i_{L_{0}}(x)\right) \leq \text { height }_{L_{0}}(\phi) .
$$

Now assume by contradiction that $L_{0} \cap L_{1}=\varnothing$. Pick a normalized Hamiltonian $H$ which is constant equal to $C_{0}$ on $L_{0}$ and constant equal to $C_{1}$ on $L_{1}$ with $C_{1}>C_{0}+K$. This immediately leads to a contradiction and concludes the proof of Theorem 2.4.1. $\square$

We now pass to the proof of Corollary 2.4.3. Put $L_{1}=L$ and let $L_{0} \subset M$ be a non-narrow monotone Lagrangian. The claim follows if we show that if $[\mathrm{pt}] *\left[L_{1}\right]$ is not divisible by $t^{2 C_{M} / N_{L_{1}}}$ in $I Q^{+}\left(L_{1}\right)$, then $j_{L_{1}} \circ i_{L_{0}} \neq 0$. We first fix a Morse function $f_{0}: L_{0} \rightarrow \mathbb{R}$ and a metric $\rho_{0}$ on $L_{0}$ as well as an almost complex structure $J$ on $M$ so that the pearl complex $\mathcal{C}\left(L_{0} ; \Lambda_{0} ; f_{0}, \rho_{0}, J\right)$ is defined. We assume that $f_{0}$ has a unique minimum $m_{0}$. To simplify the notation, we put $c_{i}=2 C_{M} / N_{L_{i}}$.

By the nondegeneracy part in Proposition 4.4.1 there exists a class $\alpha \in Q H_{0}\left(L_{0} ; \Lambda_{0}\right)$ which is nonzero and is represented by a pearl cycle of the form $m_{0}+\sum_{i>0} x_{i} t_{0}^{i}$ with $x_{i} \in \operatorname{Crit}\left(f_{0}\right)$. A priori this cycle belongs to $\mathcal{C}\left(L_{0} ; f_{0}, \rho_{0}, J\right)$, but as $\left|m_{0}\right|=0$ and $\left|t_{0}\right|<0$ all the powers of $t_{0}$ in this cycle must be nonnegative. Thus, in fact this cycle is in $\mathcal{C}^{+}\left(L_{0} ; f_{0}, \rho_{0}, J\right)$ and $\alpha \in I Q^{+}\left(L_{0}\right)$. In view of the coefficients extension morphisms $Q H\left(L_{0} ; \Lambda_{0}^{+}\right) \rightarrow Q H\left(L ; \Lambda_{0,1}^{+}\right) \rightarrow Q H\left(L ; \Lambda_{0,1}\right)$ we will view from now on $\alpha$ as an element of the image of these maps ie $\alpha \in I Q^{+}\left(L_{0} ; \Lambda_{0,1}\right) \subset Q H\left(L_{0} ; \Lambda_{0,1}\right)$. Here we have used again the ring $\Lambda_{0,1}^{+}=\Lambda_{0}^{+} \otimes_{\Gamma^{+}} \Lambda_{1}^{+} \cong \mathbb{Z}_{2}\left[t_{0}, t_{1}\right] /\left\{t_{0}^{c_{0}}=t_{1}^{c_{1}}\right\}$ and the coefficients extension morphisms induced by the obvious inclusions $\Lambda_{0}^{+} \rightarrow \Lambda_{0,1}^{+} \rightarrow$ $\Lambda_{0,1}$.

As $i_{L_{0}}$ extends (at the chain level) the inclusion in singular homology we can write $i_{L_{0}}(\alpha)=[\mathrm{pt}]+\sum_{j>0} a_{j} t_{0}^{j}$ with $a_{j} \in H_{*}\left(M ; \mathbb{Z}_{2}\right)$. Notice that $Q H\left(L_{1} ; \Lambda_{0,1}\right)=$ $Q H\left(L_{1} ; \Lambda_{1}\right) \otimes_{\Gamma} \Lambda_{0}$ as $\mathcal{C}\left(L_{1} ; \Lambda_{0,1} ; f_{1}, \rho_{1}, J\right)=\left(\mathcal{C}\left(L_{1} ; \Lambda_{1} ; f_{1}, \rho_{1}, J\right) \otimes_{\Gamma} \Lambda_{0}, d_{\Lambda_{1}} \otimes\right.$ id) and $\Lambda_{0,1}$ is a free $\Gamma$-module. Taking this into account, we now apply $j_{L_{1}}$ to $i_{L_{0}}(\alpha)$ and we obtain

$$
\left(j_{L_{1}} \circ i_{L_{0}}\right)(\alpha)=y+\sum_{j>0} y_{j} t_{0}^{j},
$$

where we have denoted $y=[\mathrm{pt}] *\left[L_{1}\right] \in Q H\left(L_{1} ; \Lambda_{1}\right) \otimes 1$ and $y_{j}=a_{j} *\left[L_{1}\right] \in$ $Q H\left(L_{1} ; \Lambda_{1}\right) \otimes 1$. It is important to notice that in fact $y, y_{j} \in I Q^{+}\left(L_{1}\right) \otimes 1 \subset$ 
$I Q^{+}\left(L_{1} ; \Lambda_{0,1}\right) \subset Q H\left(L_{1} ; \Lambda_{0,1}\right)$. Now suppose by contradiction that $j_{L_{1}} \circ i_{L_{0}}(\alpha)=0$. As $y \in I Q^{+}\left(L_{1}\right) \otimes 1$ identity (44) implies that the second term on its right-hand side belongs to $I Q^{+}\left(L_{1}\right) \otimes 1$. This can only happen if for every $j$ with $y_{j} \neq 0$ we have $c_{0} \mid j$, so that $t_{0}^{j}=\left(t_{1}^{c_{1}}\right)^{j / c_{0}}$. It now follows that $y$ is divisible by $t_{1}^{c_{1}}$, and obviously this divisibility property continues to hold also in $I^{+}\left(L_{1}\right)$. A contradiction.

\section{Various examples and computations}

The first three subsections below contain the proofs of the computational theorems in Section 2.3 and of their corollaries from Section 1.2.5. The last subsection contains the justification of Example 1.2.10.

\subsection{Lagrangians in $\mathbb{C P}^{n}$ with $2 H_{1}(L ; \mathbb{Z})=0$}

Here we prove Theorem 2.3.1 and its Corollary 1.2.11.

We recall our notation: we denote by $h=\left[\mathbb{C} P^{n-1}\right] \in H_{2 n-2}\left(\mathbb{C} P^{n} ; \mathbb{Z}_{2}\right)$ the class of a hyperplane so that in the quantum homology $Q H\left(\mathbb{C} P^{n}\right)$ we have

$$
h^{* j}= \begin{cases}h^{\cap j}, & 0 \leq j \leq n, \\ {\left[\mathbb{C} P^{n}\right] s,} & j=n+1 .\end{cases}
$$

We will use quantum homology with coefficients in $\Lambda=\mathbb{Z}_{2}\left[t^{-1}, t\right]$ and so we recall that $Q H\left(\mathbb{C} P^{n} ; \Lambda\right)=Q H\left(\mathbb{C} P^{n}\right) \otimes_{\Gamma} \Lambda$, where $\Gamma=\mathbb{Z}_{2}\left[s^{-1}, s\right], \operatorname{deg} s=-(2 n+2)$, and $\Lambda$ becomes a $\Gamma$-module by $s \rightarrow t^{(2 n+2) / N_{L}}$. Obviously, $h$ is invertible in $Q H\left(\mathbb{C} P^{n}\right)$ so that the existence of the module action claimed in Theorem A directly implies the first part of:

Lemma 6.1.1 Let $L \subset \mathbb{C} P^{n}$ be a monotone Lagrangian with $N_{L} \geq 2$. Then $Q H_{*}(L)$ is 2-periodic, ie $Q H_{i}(L) \cong Q H_{i-2}(L)$ for every $i \in \mathbb{Z}$ and the homomorphism $Q H_{i}(L) \rightarrow Q H_{i-2}(L)$ given by $\alpha \mapsto h * \alpha$ is an isomorphism for every $i \in \mathbb{Z}$. Moreover, $H_{1}(L ; \mathbb{Z}) \neq 0$.

Proof The only part that still needs to be justified is that $H_{1}(L ; \mathbb{Z}) \neq 0$. But if $H_{1}(L ; \mathbb{Z})=0$, then $N_{L}=2 C_{\mathbb{C} P^{n}}=2 n+2$ and by Theorem 1.2.2 (i) we deduce that $L$ is wide (take $l=n$ in that theorem). The first part of the lemma implies in this case that $Q H_{*}(L) \cong\left(H\left(L ; \mathbb{Z}_{2}\right) \otimes \Lambda\right)_{*}$ is 2-periodic which is impossible by degree reasons. Indeed, $\left(H\left(L ; \mathbb{Z}_{2}\right) \otimes \Lambda\right)_{n} \neq 0$ but as $|t|=-2 n-2$ we have $\left(H\left(L ; \mathbb{Z}_{2}\right) \otimes \Lambda\right)_{n+2} \cong H_{n+2}\left(L ; \mathbb{Z}_{2}\right)=0$. 
Remark 6.1.2 The first part of Lemma 6.1.1 was proved before by Seidel using the theory of graded Lagrangian submanifolds [51]. The 2-periodicity in [51] follows from the fact that $\mathbb{C} P^{n}$ admits a Hamiltonian circle action which induces a shift by 2 on graded Lagrangian submanifolds. Note that this is compatible with our perspective since that $S^{1}$-action gives rise to an invertible element in $Q H\left(\mathbb{C} P^{n}\right)$ (the Seidel element [50; 40]) whose degree is exactly $2 n$ minus the shift induced by the $S^{1}$-action. In our case the Seidel element turns out to be $h$.

We now focus on our main object of interest in the subsection.

Lemma 6.1.3 Let $L$ be a Lagrangian submanifold in $\mathbb{C} P^{n}$. If $2 H_{1}(L ; \mathbb{Z})=0$ then $L$ is monotone, $N_{L}=n+1, L$ is wide and as a graded vector space we have $H_{*}\left(L ; \mathbb{Z}_{2}\right) \cong H_{*}\left(\mathbb{R} P^{n} ; \mathbb{Z}_{2}\right)$. Moreover, $Q H_{i}(L) \cong \mathbb{Z}_{2}$ for every $i \in \mathbb{Z}$.

Proof Since $2 H_{1}(L ; \mathbb{Z})=0$ it is easy to see that $L$ is monotone. Moreover, a simple computation shows that the minimal Maslov number of $L$ is $N_{L}=k(n+1)$ with $k \in\{1,2\}$. We already know from Lemma 6.1 .1 that $H_{1}\left(L ; \mathbb{Z}_{2}\right) \neq 0$ so that $H_{*}\left(L ; \mathbb{Z}_{2}\right)$ is generated as an algebra by $H_{\geq 1}\left(L ; \mathbb{Z}_{2}\right)$. Thus, by Theorem 1.2.2 (i), $L$ is wide so that, again by Lemma 6.1.1, we deduce that $\left(H\left(L ; \mathbb{Z}_{2}\right) \otimes \Lambda\right)_{*}$ is 2 -periodic. This 2-periodicity implies (for degree reasons) that $N_{L}$ cannot be $2(n+1)$, hence $k=1$ and $N_{L}=n+1$. Moreover the 2-periodicity implies that $H_{2 i}\left(L ; \mathbb{Z}_{2}\right) \cong H_{0}\left(L ; \mathbb{Z}_{2}\right)=\mathbb{Z}_{2}$ for every $0 \leq 2 i \leq n$. Similarly we have: $H_{1}\left(L ; \mathbb{Z}_{2}\right) \cong Q H_{1}(L) \cong Q H_{1}(L) t^{-1}=$ $Q H_{n+2}(L) \cong Q H_{n}(L) \cong H_{n}\left(L ; \mathbb{Z}_{2}\right)=\mathbb{Z}_{2}$. Applying the 2 -periodicity again we obtain $H_{2 i+1}\left(L ; \mathbb{Z}_{2}\right) \cong \mathbb{Z}_{2}$ for every $1 \leq 2 i+1 \leq n$. Summing up we see that $H_{j}\left(L ; \mathbb{Z}_{2}\right) \cong \mathbb{Z}_{2} \cong H_{j}\left(\mathbb{R} P^{n} ; \mathbb{Z}_{2}\right)$ for every $0 \leq j \leq n$.

As for the last statement regarding $Q H_{i}(L)$, we have

$$
\begin{aligned}
Q H_{2 j}(L) & \cong Q H_{0}(L) \cong H_{0}\left(L ; \mathbb{Z}_{2}\right)=\mathbb{Z}_{2}, \\
Q H_{2 j+1}(L) & \cong Q H_{1}(L) \cong H_{1}\left(L ; \mathbb{Z}_{2}\right) \cong \mathbb{Z}_{2} .
\end{aligned}
$$

Lemma 6.1.4 There is a map $\phi: L \rightarrow \mathbb{R} P^{n}$ inducing an isomorphism in $\mathbb{Z}_{2}$-singular homology. In particular $H_{*}\left(L ; \mathbb{Z}_{2}\right)$ is isomorphic to $H_{*}\left(\mathbb{R} P^{n} ; \mathbb{Z}_{2}\right)$ as an algebra. Moreover, the isomorphism $\phi_{*}$ identifies the classical external product $H_{*}\left(\mathbb{C} P^{n} ; \mathbb{Z}_{2}\right) \otimes$ $H_{*}\left(L ; \mathbb{Z}_{2}\right) \rightarrow H_{*}\left(L ; \mathbb{Z}_{2}\right)$ with the corresponding action for $\mathbb{R} P^{n} \subset \mathbb{C} P^{n}$.

Proof Let $\alpha_{i} \in Q H_{i}(L) \cong \mathbb{Z}_{2}$ be the generator. In view of the canonical isomorphism $Q H_{*}(L) \cong\left(H\left(L ; \mathbb{Z}_{2}\right) \otimes \Lambda\right)_{*}$ we have $H_{j}\left(L ; \mathbb{Z}_{2}\right) \cong Q H_{j}(L)$ for every $0 \leq j \leq n$. Therefore we will view $\alpha_{j}, 0 \leq j \leq n$, also as elements of $H_{j}\left(L ; \mathbb{Z}_{2}\right)$. 
We first claim that $\alpha_{n-1} * \alpha_{n-1}=\alpha_{n-1} \cdot \alpha_{n-1}=\alpha_{n-2}$ (where $-\cdot-$ is the classical intersection product). For degree reasons this is equivalent to $\alpha_{n-1} \cdot \alpha_{n-1} \neq 0$. In turn, this is equivalent to showing that $\alpha^{1} \cup \alpha^{1} \neq 0$ in $H^{2}\left(L ; \mathbb{Z}_{2}\right)$ where $\alpha^{1} \in H^{1}\left(L ; \mathbb{Z}_{2}\right)$ is the generator (and so is Poincaré dual to $\left.\alpha_{n-1}\right)$. From the fact that $H^{1}\left(L ; \mathbb{Z}_{2}\right)=\mathbb{Z}_{2}$ and $H_{1}(L ; \mathbb{Z})$ is 2 -torsion we obtain that the Bockstein homomorphism, $\beta: H^{1}\left(L ; \mathbb{Z}_{2}\right) \rightarrow$ $H^{2}\left(L ; \mathbb{Z}_{2}\right)$, associated to the exact sequence $0 \rightarrow \mathbb{Z}_{2} \rightarrow \mathbb{Z}_{4} \rightarrow \mathbb{Z}_{2} \rightarrow 0$ is not trivial. But $\beta=\mathrm{Sq}^{1}$, the first Steenrod square, which in this degree coincides with the square cup-product, so that $\alpha^{1} \cup \alpha^{1} \neq 0$. This proves that $\alpha_{n-1} * \alpha_{n-1}=\alpha_{n-1} \cdot \alpha_{n-1}=\alpha_{n-2}$.

In view of the first part of Lemma 6.1.1 we know that $h * \alpha_{i}=\alpha_{i-2}$ for all $i$. As $\alpha_{n-1} \cdot \alpha_{n-1}=\alpha_{n-2}$ it follows that the $\mathbb{Z}_{2}$-singular homology of $L$ coincides as an algebra with that of $\mathbb{R} P^{n}$. Let $\bar{\phi}: L \rightarrow \mathbb{R} P^{\infty}$ be the classifying map associated to $\alpha^{1}$. As $\operatorname{dim}(L)=n$ we deduce that $\bar{\phi}$ factors via a map $\phi: L \rightarrow \mathbb{R} P^{n}$ and as the induced map in cohomology $H^{1}(\phi): H^{1}\left(\mathbb{R} P^{n} ; \mathbb{Z}_{2}\right) \rightarrow H^{1}\left(L ; \mathbb{Z}_{2}\right)$ is an isomorphism it follows that $\phi$ induces an isomorphism in homology in all degrees. Moreover, using the relation $h * \alpha_{i}=\alpha_{i-2}$ again, we deduce that the classical external product coincides with that for $\mathbb{R} P^{n}$.

We now turn to the proof of Theorem 2.3.1. Point (ii) has already been proved (in the proof of Lemma 6.1.4). Before we go on, recall that we have denoted by $\alpha_{i} \in Q H_{i}(L) \cong \mathbb{Z}_{2}$ the generator. Clearly we have $\alpha_{i-r(n+1)}=\alpha_{i} t^{r}$ for every $i, r \in \mathbb{Z}$.

Another important fact we will need below is the following. By Theorem A the quantum inclusion $i_{L}: Q H(L) \rightarrow Q H(M ; \Lambda)$ is determined by the module action and the augmentation $\epsilon_{L}$ via the formula

$$
\left\langle\mathrm{PD}(y), i_{L}(x)\right\rangle=\epsilon_{L}(y * x) .
$$

We are now ready to prove points (iii) and (iv) of Theorem 2.3.1. Assume first that $n$ is even, $n=2 l$. Denote by $h_{2 r} \in H_{2 r}\left(\mathbb{C} P^{n} ; \mathbb{Z}_{2}\right)$ the generator, so that $h_{2 n-2}=h$ and $h_{2 r}=h^{*(n-r)}$ for every $0 \leq r \leq n$. Fix $0 \leq 2 k \leq n$. For degree reasons we have $i_{L}\left(\alpha_{2 k}\right)=e h_{2 k}$ for some $e \in \mathbb{Z}_{2}$. Applying (45) with $x=\alpha_{2 k}$ and $y=h_{2 n-2 k}$ we obtain

$$
e=\epsilon_{L}\left(h_{2 n-2 k} * \alpha_{2 k}\right)=\epsilon_{L}\left(h^{* k} * \alpha_{2 k}\right)=\epsilon_{L}\left(\alpha_{0}\right)=1 .
$$

Now fix $1 \leq 2 k+1 \leq n-1$. For degree reasons, $i_{L}\left(\alpha_{2 k+1}\right)=f h_{2 k+n+2} t$ for some $f \in \mathbb{Z}_{2}$. Applying (45) with $x=\alpha_{2 k+1}, y=h_{n-2 k-2}$ we obtain $f t=\epsilon_{L}\left(h_{n-2 k-2} * \alpha_{2 k+1}\right)=\epsilon_{L}\left(h^{*(k+1+l)} * \alpha_{2 k+1}\right)=\epsilon_{L}\left(\alpha_{-2 l-1}\right)=\epsilon_{L}\left(\alpha_{0} t\right)=t$,

hence $f=1$. This concludes the proof for even $n$. The case $n=$ odd is very similar, so we omit the details. 
It remains to prove point (i) of Theorem 2.3.1. For this end, first notice that since $[L]=\alpha_{n}$ we have $\alpha_{n-2}=h *[L]$. As both $[L] \in Q H(L)$ and $h \in Q H\left(\mathbb{C} P^{n} ; \Lambda\right)$ are invertible (each in its respective ring) it follows that $\alpha_{n-2}$ is invertible too. By the proof of Lemma 6.1.4 we have $\alpha_{n-2}=\alpha_{n-1} * \alpha_{n-1}$, hence $\alpha_{n-1}$ is invertible too. It follows that $\left(\alpha_{n-1}\right)^{*(n-i)} \neq 0 \in Q H_{i}(L)$, hence $\alpha_{i}=\left(\alpha_{n-1}\right)^{*(n-i)}$. As this is true for every $i \in \mathbb{Z}$ the claim at point (i) of Theorem 2.3.1 readily follows. This concludes the proof of all the statements of Theorem 2.3.1

We now turn to proving Corollary 1.2.11. We begin with point (iv). This follows easily from points (iii) and (iv) of Theorem 2.3.1 by looking at the classical part of the quantum inclusion $Q H_{*}(L) \rightarrow Q H_{*}\left(\mathbb{C} P^{n} ; \Lambda\right)$. Point (iii) follows in a similar way from the fact that $h *[L]=\alpha_{n-2}$.

As point (i) and (ii) of Corollary 1.2.11 has already been proved it now remains to prove points (v), (vi) and (vii) of that corollary. We group these in the next lemma.

Lemma 6.1.5 For a Lagrangian $L$ in $\mathbb{C} P^{n}$ with $2 H_{1}(L ; \mathbb{Z})=0$ we have:

- $\left(\mathbb{C} P^{n}, L\right)$ is $(1,0)$-uniruled of order $n+1$.

- $L$ is 2 -uniruled of order $n+1$. Moreover, given two distinct points $x, y \in L$, for a generic $J$ there is an even but nonvanishing number of disks of Maslov index $n+1$ whose boundary passes through these two points.

- For $n=2,\left(\mathbb{C} P^{2}, L\right)$ is $(1,2)$-uniruled of order 6 .

Proof Fix a Morse function $f: L \rightarrow \mathbb{R}$ with a single minimum and a single maximum and fix also a perfect Morse function $g: \mathbb{C} P^{n} \rightarrow \mathbb{R}$. Fix also Riemannian metrics $\rho_{L}$ on $L$ and $\rho_{M}$ on $M=\mathbb{C} P^{n}$ as well as an almost complex structure $J$ so that the pearl complex $\mathcal{C}(f)=\mathcal{C}\left(L ; \Lambda ; f, \rho_{L}, J\right)$ and the Morse complex (tensored with $\Lambda$ ) $C(g)$ are defined as well as the module product

$$
C(g) \otimes \mathcal{C}(f) \rightarrow \mathcal{C}(f) .
$$

Let $f^{\prime}: L \rightarrow \mathbb{R}$ be a second Morse function (again with a single minimum and maximum) and assume that the pearl complex $\mathcal{C}\left(f^{\prime}\right)=\mathcal{C}\left(L ; \Lambda ; f^{\prime}, \rho_{L}, J\right)$ is defined as well as the quantum product:

$$
\mathcal{C}\left(f^{\prime}\right) \otimes \mathcal{C}(f) \rightarrow \mathcal{C}(f) .
$$

We now prove point (i). We have the relation

$$
[\mathrm{pt}] * \alpha_{n}=h^{*_{n}} * \alpha_{n}=\alpha_{-n}=\alpha_{1} t \in Q H(L)
$$

where, as before, $h \in H_{2 n-2}\left(\mathbb{C} P^{n} ; \mathbb{Z}_{2}\right)$ is the generator. Denote by $w$ the maximum of $f$ and by $p$ the minimum of $g$. The critical point $w$ is a cycle in $\mathcal{C}(f)$ and $[w]=\alpha_{n}$. 
Thus, in view of relation (46) we have $p * w \neq 0 \in \mathcal{C}_{-n}(f)$. As $\mathcal{C}_{-n}(f)=\mathcal{C}_{1}(f) t=$ $\mathbb{Z}_{2}\langle\operatorname{Crit}(f)\rangle t$ (the last equality being true for degree reasons) we obtain that $p * w$ has a summand which is of the type $y t$, where $y \in \operatorname{Crit}_{1}(f)$. Given the definition of the module action in Section 3.2 (c) this means that there is a $J$-disk of Maslov index $n+1$ through the point $p$. As we may choose $g$ so that the point $p$ is anywhere desired in $\mathbb{C} P^{n} \backslash L$ this implies point (i).

For point (ii) we will use the relation

$$
\alpha_{n-1} * \alpha_{0}=\alpha_{n} t
$$

To exploit this we denote by $m$ the minimum of $f$ and we let $c$ be a cycle in $\mathcal{C}\left(f^{\prime}\right)$ which represents $\alpha_{n-1}$. Because $L$ is wide, $m$ is a Morse cycle and $N_{L}=n+1$, we deduce that $m$ is also a cycle in $\mathcal{C}(f)$ so that $[m]=\alpha_{0}$. Thus we have, at the chain level, $c * m=w t$. In view of the definition of the quantum product in Section $3.2(\mathrm{~b})$, we deduce that for generic $J$ there exists a $J$-disk of Maslov index $n+1$ through both $w$ and $m$. To finish with this point we need now to remark that the number $n(m, w)$ of such disks is even. Indeed, if $d$ is the differential of the pearl complex $\mathcal{C}(f)$, notice that for degree reasons the differential of $m$ has the form $d m=\epsilon w t$ where $\epsilon \in \mathbb{Z}_{2}$ is the parity of $n(m, w)$. But, as mentioned above, $L$ is wide and so $\epsilon=0$.

For the third point we use the relation

$$
[\mathrm{pt}] * \alpha_{0}=\alpha_{2} t^{2}
$$

and the fact that, when $n=2, \alpha_{2}=[w]$. At the chain level (48) becomes $p * m=w t^{2}$. By interpreting this relation in terms of the moduli spaces used in Section 3.2 (c) to define the module product we deduce that there is a "chain of pearls" of one of the following types:

- two disks $u_{1}, u_{2}$ joined by a flow line of $-\nabla f$ so that $m \in u_{1}(\partial D), w \in u_{2}(\partial D)$, $\mu\left(u_{1}\right)=\mu\left(u_{2}\right)=3$ and $p$ belongs to the image of one of the $\left.u_{i}\right|_{\text {Int } D}$ 's,

- a single disk $u$ of Maslov index $2 n+2=6$ whose interior goes through $p$ and with $m, w \in u(\partial D)$.

Notice that given two points $k \in \mathbb{C} P^{n} \backslash L$, and $k^{\prime} \in L$, for a generic $J$, there is no disk of Maslov index $n+1$ passing through both $k$ and $k^{\prime}$ because the virtual dimension of the moduli spaces of such disks equals -1 . Thus generically, case (a) is not possible and so we are left with case (b) which proves claim (iii) of the lemma. 


\subsection{The Clifford torus}

This subsection consists of a sequence of results in which we prove all the properties claimed in Theorem 2.3.2 and Corollary 1.2.12.

\section{Lemma 6.2.1 The Clifford torus $\mathbb{T}_{\text {clif }}^{n} \in \mathbb{C} P^{n}$ is wide and $N_{\mathbb{T}_{\text {clif }}^{n}}=2$.}

This Lemma was first proved by Cho [21] by a direct computation. Below we give a somewhat different proof.

Proof We first notice that by Theorem 1.2.2 any Lagrangian torus $L$ is narrow or wide and if $N_{L} \geq 3$, then it is wide. In the case of the Clifford torus, $\mathbb{T}_{\text {clif }}^{n}=\left\{\left[z_{0}: \cdots: z_{n}\right] \in\right.$ $\left.\mathbb{C} P^{n}|| z_{0}|=\cdots=| z_{n} \mid\right\} \subset \mathbb{C} P^{n}$, a simple computation shows that it is monotone and that $N_{\mathbb{T}_{\text {clif }}}=2$. Moreover (see Cho [21]), with the standard complex structure on $\mathbb{C} P^{n}$ there are exactly $n+1$ families of disks of Maslov index 2 with boundary on $\mathbb{T}_{\text {clif }}^{n}$, $\gamma_{0}, \gamma_{1}, \ldots, \gamma_{n}$ so that for any point $x \in \mathbb{T}_{\text {clif }}^{n}$ there is precisely one disk $\Delta_{i}(x)$ from the family $\gamma_{i}$ passing through $x$. In fact we can describe these disks explicitly as follows. Write $x=\left[x_{0}: \cdots: x_{n}\right] \in \mathbb{T}_{\text {clif }}^{n}$ with $\left|x_{i}\right|=1$ for every $i$. Then the disk $\Delta_{i}(x)$ is given by $D \ni z \mapsto\left[x_{0}: \cdots: x_{i-1}: z: x_{i+1}: \cdots: x_{n}\right] \in \mathbb{C} P^{n}$.

It is proved in [21] that these disks are regular and we can choose a basis of $H_{1}\left(\mathbb{T}_{\text {clif }}^{n} ; \mathbb{Z}\right)$ represented by the curves $c_{i}=\partial\left(\Delta_{i}(x)\right), 1 \leq i \leq n$. In this basis, $c_{0}=\partial\left(\Delta_{0}(x)\right) \simeq$ $-c_{1}-c_{2}-\cdots-c_{n}$. Using the criterion for the vanishing of Floer homology in Proposition 4.2.1 we see that the cycle $D_{1}$ defined there is null-homologous and so $\mathbb{T}_{\text {clif }}^{n}$ is wide.

For the 2-dimensional Clifford torus we now pass to verifying the properties of the quantum product as stated in Theorem 2.3.2. Before we go into these computations recall from Section 4.5 that although $\mathbb{T}_{\text {clif }}^{2}$ is wide there might not be a canonical isomorphism $H\left(\mathbb{T}_{\text {clif }}^{2} ; \mathbb{Z}_{2}\right) \otimes \Lambda \cong Q H\left(\mathbb{T}_{\text {clif }}^{2}\right)$. This turns out to be indeed the case (see Biran and Cornea $[13 ; 12])$. However, by Proposition 4.5 .1 we have canonical embeddings $H_{1}\left(\mathbb{T}_{\text {clif }}^{2} ; \mathbb{Z}_{2}\right) \otimes \Lambda_{*} \hookrightarrow Q H_{1+*}\left(\mathbb{T}_{\text {clif }}^{2}\right)$ and $H_{2}\left(\mathbb{T}_{\text {clif }}^{2} ; \mathbb{Z}_{2}\right) \otimes \Lambda_{*} \hookrightarrow Q H_{2+*}\left(\mathbb{T}_{\text {clif }}^{2}\right)$. This implies, for degree reasons, that

$$
Q H_{1}\left(\mathbb{T}_{\text {clif }}^{2}\right) \cong H_{1}\left(\mathbb{T}_{\text {clif }}^{2} ; \mathbb{Z}_{2}\right), \quad Q H_{0}\left(\mathbb{T}_{\text {clif }}^{2}\right) \cong H_{0}\left(\mathbb{T}_{\text {clif }} ; \mathbb{Z}_{2}\right) \oplus\left[\mathbb{T}_{\text {clif }}^{2}\right] t,
$$

where the first isomorphism is canonical and the second isomorphism is not canonical but the second summand on its right-hand side (involving the fundamental class $\left[\mathbb{T}_{\text {clif }}^{2}\right] t$ ) is canonical.

In view of (49), let $w=\left[\mathbb{T}_{\text {clif }}^{2}\right] \in H_{2}\left(\mathbb{T}_{\text {clif }}^{2} ; \mathbb{Z}_{2}\right)$ be the fundamental class and let $a=$ $\left[c_{1}\right], b=\left[c_{2}\right] \in H_{1}\left(\mathbb{T}_{\text {clif }}^{2} ; \mathbb{Z}_{2}\right) \cong Q H_{1}\left(\mathbb{T}_{\text {clif }}^{2}\right)$. By the preceding discussion $w, a, b$ can be viewed as well defined elements of $Q H\left(\mathbb{T}_{\text {clif }}^{2}\right)$. 
Lemma 6.2.2 There is an element $m \in Q H_{0}\left(\mathbb{T}_{\text {clif }}^{2}\right)$ which together with $w t$ generates $Q H_{0}\left(\mathbb{T}_{\text {clif }}^{2}\right)$ so that we have $a * b=m+w t, b * a=m, a * a=b * b=w t$, $m * m=m t+w t^{2}$.

Proof We consider a perfect Morse function $f: \mathbb{T}_{\text {clif }}^{2} \rightarrow \mathbb{R}$ and, by a slight abuse in notation, we denote its minimum by $m$. Similarly, we denote its maximum by $w$ and we let the two critical points of index 1 be denoted by $a^{\prime}$ and $b^{\prime}$. We pick $f$ so that the closure of the unstable manifold of $a^{\prime}$ represents $a \in H_{1}\left(\mathbb{T}_{\text {clif }}^{2} ; \mathbb{Z}_{2}\right)$ and the unstable manifold of the critical point $b^{\prime}$ represents $b$.

To simplify notation we denote the disk $\Delta_{i}(w)$ by $d_{i}$. See Figure 7 . By possibly

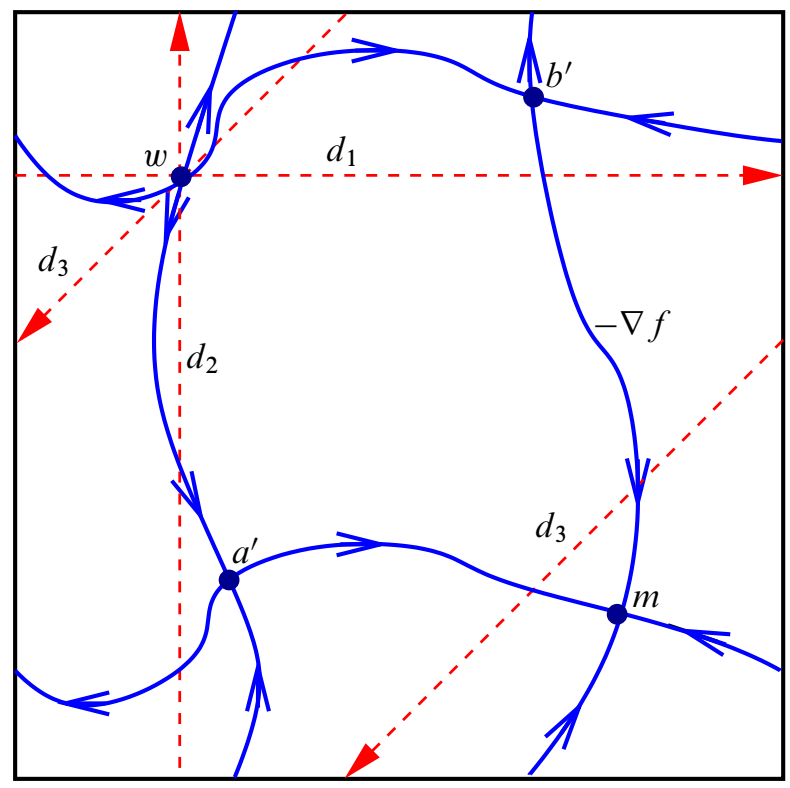

Figure 7: Trajectories of $-\nabla f$ and holomorphic disks on $\mathbb{T}_{\text {clif }}^{2}$

perturbing the function $f$ slightly we may assume that the unstable manifold of $a^{\prime}$ intersects $d_{2}$ and $d_{3}$ in a single point and is disjoint from $d_{1}$. Similarly, we may assume that the unstable manifold of $b^{\prime}$ intersects $d_{1}$ and $d_{3}$ at a single point and that this unstable manifold is disjoint from $d_{2}$. With these choices the pearl complex $(\mathcal{C}(f, J, \rho), d)$ is well defined. Here we take $J$ to be the standard complex structure of $\mathbb{C} P^{2}$, or a generic small perturbation of it and $\rho$ a generic small perturbation of the flat metric on $\mathbb{T}_{\text {clif }}^{2}$. As $f$ is perfect and $\mathbb{T}_{\text {clif }}^{2}$ is wide, the differential in $\mathcal{C}(f, J, \rho)$ vanishes. From now on we will view $m, a^{\prime}, b^{\prime}, w$ as generators (over $\Lambda$ ) of $Q H_{*}\left(\mathbb{T}_{\text {clif }}^{2}\right.$ ). Recall that $m$ depends on the choice of $f$ in the sense that if we take another perfect 
Morse function $\tilde{f}$ with minimum $\tilde{m}$, then $\tilde{m}$ might give an element of $Q H_{0}\left(\mathbb{T}_{\text {clif }}^{2}\right)$ which is different from $m$. On the other hand $a^{\prime}, b^{\prime}, w \in Q H$ are canonical.

In order to compute the various products of $a^{\prime}$ and $b^{\prime}$ we use another perfect Morse function $g: \mathbb{T}_{\text {clif }}^{2} \rightarrow \mathbb{R}$ with critical points $a^{\prime \prime}, b^{\prime \prime}, m^{\prime \prime}, w^{\prime \prime}$. We may choose $g$ to be a small perturbation of $f$ so that the unstable and stable manifolds of $a^{\prime \prime}, b^{\prime \prime}$ become "parallel" copies of those of the corresponding points of $f$ (see Figure 8). Moreover, by taking $g$ to be close enough to $f$ (and keeping $J$ and $\rho$ fixed) we may assume that the comparison chain map $\Psi^{\mathrm{prl}}=\Psi_{(f, \rho, J),(g, \rho, J)}: \mathcal{C}(L ; f, \rho, J) \rightarrow \mathcal{C}(L ; g, \rho, J)$ coincides with the Morse comparison chain map $\Psi_{(f, \rho),(g, \rho)}^{\mathrm{Morse}}$, namely,

$$
\Psi^{\mathrm{prl}}\left(a^{\prime}\right)=a^{\prime \prime}, \quad \Psi^{\mathrm{prl}}\left(b^{\prime}\right)=b^{\prime \prime}, \quad \Psi^{\mathrm{prl}}(m)=m^{\prime \prime}, \quad \Psi^{\mathrm{prl}}(w)=w^{\prime \prime} .
$$

See point (e) in Section 3.2 as well as the proof of Proposition 4.4.1 for various descriptions of the comparison map $\Psi^{\mathrm{prl}}$ (this map was denoted in the proof of Proposition 4.4.1 by $\left.\phi^{f, f^{\prime}}\right)$.

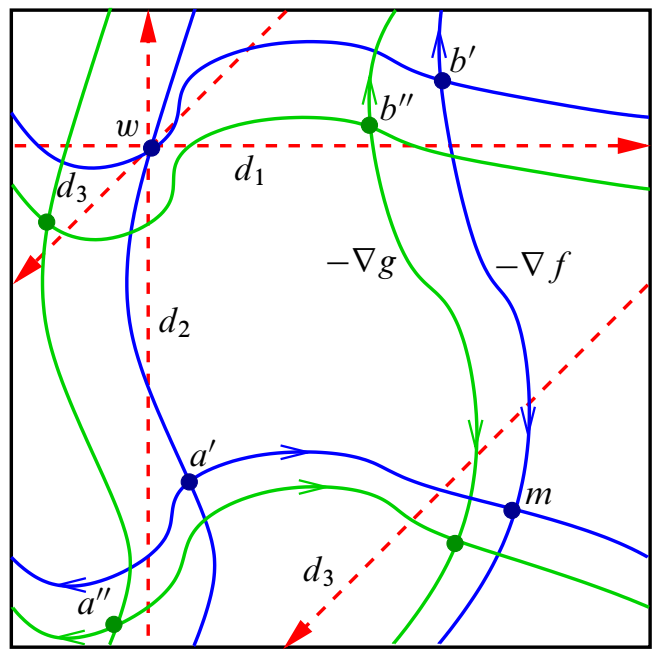

Figure 8: Trajectories of $-\nabla f,-\nabla g$ and holomorphic disks on $\mathbb{T}_{\text {clif }}^{2}$

We now compute the product (on the chain level)

$$
*: \mathcal{C}(L ; f, \rho, J) \otimes \mathcal{C}(L ; g, \rho, J) \rightarrow \mathcal{C}(L ; f, \rho, J) .
$$

For degree reasons we have

$$
a^{\prime} * b^{\prime \prime}=m+\epsilon w t, \quad b^{\prime} * a^{\prime \prime}=m+\epsilon^{\prime} w t, \quad \text { for some } \epsilon, \epsilon^{\prime} \in \mathbb{Z}_{2} .
$$


By the definition of the quantum product, $\epsilon$ is the number modulo 2 of $J$-disks with $\mu=2$ going - in clockwise order ! - through the following points: one point in the unstable manifold of $a^{\prime}$ then $w$ and, finally one point in the unstable manifold of $b^{\prime \prime}$. Similarly, $\epsilon^{\prime}$ is the number modulo 2 of disks with $\mu=2$ going in order through a point in the unstable manifold of $b^{\prime}, w$ and then a point in the unstable manifold of $a^{\prime \prime}$. There is a single disk through $w$ which also intersects both the unstable manifolds of $a^{\prime}$ and $b^{\prime}-$ the disk $d_{3}$. However, the order in which the three types of points appear on the boundary of this disk implies that precisely one of $\epsilon$ and $\epsilon^{\prime}$ is nonzero. Looking at Figure 8 we see that for our choices of Morse data and $J$ we actually have $\epsilon=1, \epsilon^{\prime}=0$. Thus $a^{\prime} * b^{\prime \prime}=m+w t, b^{\prime} * a^{\prime \prime}=m$, hence in $Q H\left(\mathbb{T}_{\text {clif }}^{2}\right)$ we have $a * b=m+w t$ and $b * a=m$.

Next we compute $a * a$ and $b * b$ via $a^{\prime} * a^{\prime \prime}$ and $b^{\prime} * b^{\prime \prime}$. To this end first note that $a^{\prime} * a^{\prime \prime}=\delta w t$ with $\delta \in\{0,1\}$ (the classical term vanishes here since in singular homology we have $a \cdot a=0$ ). There are precisely two pseudo-holomorphic disks that go through $w$ as well as through both unstable manifolds of $a^{\prime}$ and of $a^{\prime \prime}$ : the disks $d_{2}$ and $d_{3}$. It is at this point that we use the fact that $\left[d_{2}\right]=b,\left[d_{3}\right]=-a-b$. Indeed, this means that the order in which these three points lie on the boundary of each of these two disks is opposite. Thus, exactly one of these disks will contribute to $\delta$ and so $\delta=1$. (In fact, looking at Figure 8 we see that the relevant disk is $d_{2}$.) A similar argument shows $b^{\prime} * b^{\prime \prime}=w t$. The formula for $m * m$ follows now from the associativity of the product. Indeed

$$
m * m=(a * b+w t) *(b * a)=a *(b * b) * a+b * a t=m t+w t^{2} .
$$

(Recall that we are working over $\mathbb{Z}_{2}$.)

Remark 6.2.3 For the $n$-dimensional Clifford torus, $\mathbb{T}_{\text {clif }}^{n} \subset \mathbb{C} P^{n}$, let $t_{1}, \ldots, t_{n}$ be a basis of $H_{n-1}\left(\mathbb{T}_{\text {clif }}^{n} ; \mathbb{Z}_{2}\right)$ dual to the basis $\left[c_{1}\right], \ldots,\left[c_{n}\right] \in H_{1}\left(\mathbb{T}_{\text {clif }}^{n} ; \mathbb{Z}_{2}\right)$, with respect to the (classical) intersection product. The same argument as that giving the product $a * b, b * a$ in the proof of the lemma above shows that for $i \neq j, t_{i} * t_{j}+t_{j} * t_{i}=w t$ where $w$ represents the fundamental class.

We now turn to determining the quantum module structure (points (ii) and (iii) in Theorem 2.3.2). We recall that $h \in H_{2}\left(\mathbb{C} P^{2} ; \mathbb{Z}_{2}\right)$ is the class of a hyperplane, hence in this case of a projective line $\mathbb{C} P^{1} \subset \mathbb{C} P^{2}$.

Lemma 6.2.4 With the notation above we have:

- $h * a=a t, h * b=b t, h * w=w t, h * m=m t$.

- $i_{L}(m)=[\mathrm{pt}]+h t+\left[\mathbb{C} P^{2}\right] t^{2}, i_{L}(a)=i_{L}(b)=i_{L}(w)=0$. 
Proof We will make use of a second geometric fact concerning the Clifford torus: there is a symplectomorphism homotopic to the identity, $\bar{\phi}: \mathbb{C} P^{2} \rightarrow \mathbb{C} P^{2}$, whose restriction to $\mathbb{T}_{\text {clif }}^{2}$ is the permutation of the two factors in $\mathbb{T}_{\text {clif }}^{2} \approx S^{1} \times S^{1}$. We now determine what is the map

$$
\tilde{\phi}: Q H_{*}\left(\mathbb{T}_{\text {clif }}^{2}\right) \rightarrow Q H_{*}\left(\mathbb{T}_{\text {clif }}^{2}\right)
$$

which is induced by $\bar{\phi}$. For degree reasons we have $\widetilde{\phi}(w)=w, \widetilde{\phi}(a)=b, \widetilde{\phi}(b)=a$ and, by Proposition 4.3.1, we know that $\tilde{\phi}$ is a morphism of algebras (from this it also follows immediately that $\tilde{\phi}(m)=m+w t)$.

We now compute $h * a$ and $h * b$. We have, $h * a=h * \tilde{\phi}(b)=\tilde{\phi}(h * b)$. Now $h * a=\left(u_{1} a+u_{2} b\right) t$ with $u_{1}, u_{2} \in \mathbb{Z}_{2}$ which implies that $h * b=\left(u_{1} b+u_{2} a\right) t$. As in Lemma 6.1.1 we also have that $h *(-): H_{1}\left(\mathbb{T}_{\text {clif }}^{2} ; \mathbb{Z}_{2}\right) \rightarrow H_{1}\left(\mathbb{T}_{\text {clif }}^{2} ; \mathbb{Z}_{2}\right) t$ is an isomorphism. This implies that precisely one of $u_{1}, u_{2}$ is non zero. Assume first that $u_{1}=0$ and $u_{2}=1$. Then $h * a=b t, h *(h * a)=a t^{2}$ and $h *(h *(h * a))=b t^{3}$ which is not possible because $h^{* 3}=\left[\mathbb{C} P^{2}\right] t^{3}$ (where, $\left[\mathbb{C} P^{2}\right]$ denotes the fundamental class of $\mathbb{C} P^{2}$ ) and $\left[\mathbb{C} P^{2}\right] * a=a$. Thus we are left with $u_{1}=1, u_{2}=0$ as claimed.

To compute $h * w$ write $h * w t=h *(a * a)=(h * a) * a=(a * a) t=w t^{2}$. Similarly $h * m=h *(b * a)=(h * b) * a=m t$.

Finally, point (ii) is an immediate consequence of the first point and of formula (6) in Theorem A (iii).

Finally, we need to justify the uniruling properties of the Clifford torus as described in Corollary 1.2.12.

Lemma 6.2.5 For $n \geq 2,\left(\mathbb{C} P^{n}, \mathbb{T}_{\text {clif }}^{n}\right)$ is $(1,0)$-uniruled of order $2 n$ and $\mathbb{T}_{\text {clif }}^{n}$ is uniruled of order 2 . For $n=2,\left(\mathbb{C} P^{2}, \mathbb{T}_{\text {clif }}^{2}\right)$ is $(1,1)$-uniruled of order 4 .

Proof As $\mathbb{T}_{\text {clif }}^{n}$ is wide of minimal Maslov number 2 and $\mathbb{C} P^{n}$ is point invertible of order $2 n+2$ we deduce from Theorem 1.2.3 that $\left(\mathbb{C} P^{n}, \mathbb{T}_{\text {clif }}^{n}\right)$ is uniruled of order (at most) $2 n$. The fact that $\mathbb{T}_{\text {clif }}^{n}$ is uniruled of order 2 follows immediately from the relation $t_{i} * t_{j}+t_{j} * t_{i}=w t$ from Remark 6.2.3. Indeed, this relation implies the existence of a disk of Maslov index 2 through $w$ (for generic $J$ ). There is also a direct proof of this, based on the fact that the families of $J$-disks $\gamma_{i}$ are regular and thus, being of minimal possible area, they persist under generic deformations of $J$. Finally, for $n=2$, with the notation in Lemma 6.2.4 we have the relation [pt] $* m=m t^{2}$ where $[\mathrm{pt}]=h^{* 2}$. We consider a Morse function $g: \mathbb{C} P^{2} \rightarrow \mathbb{R}$ which is perfect and we denote its minimum by $p$. The previous relation gives (at the chain level): $p * m=m t^{2}$ where $m$ is the minimum of a perfect Morse function $f: \mathbb{T}_{\text {clif }}^{2} \rightarrow \mathbb{R}$ (so that the respective 
pearl complex and all the relevant operations are defined). This means that there is a configuration consisting of one of the following:

(a) one $J$-disk with $\mu=4$ through $p$, whose boundary is on $\mathbb{T}_{\text {clif }}^{2}$ and contains $m$,

(b) two $J$-disks, each with $\mu=2$, related by a negative gradient flow line of $f$ so that one of these two disks goes through $p$ and the boundary of the other contains $m$.

To prove our claim we only have to notice that possibility (b) cannot arise for a generic $J$. Indeed, generically, the set of points in $\mathbb{C} P^{2}$ which lie in the image of some $J$-disk of Maslov index 2 is only 3 -dimensional and so, generically, these disks avoid $p$.

\subsection{Lagrangians in the quadric}

Here we prove Theorem 2.3.4 and Corollary 1.2.13.

Let $Q \subset \mathbb{C} P^{n+1}$ be a smooth complex $n$-dimensional quadric, where $n \geq 2$. More specifically we can write $Q$ as the zero locus $Q=\left\{z \in \mathbb{C} P^{n+1} \mid q(z)=0\right\}$ of a homogeneous quadratic polynomial $q$ in the variables $\left[z_{0}: \cdots: z_{n+1}\right] \in \mathbb{C} P^{n+1}$, where $q$ defines a quadratic form of maximal rank. We endow $Q$ with the symplectic structure induced from $\mathbb{C} P^{n+1}$. (Recall that we use the normalization that the symplectic structure $\omega_{\mathrm{FS}}$ of $\mathbb{C} P^{n+1}$ satisfies $\int_{\mathbb{C} P^{1}} \omega_{\mathrm{FS}}=1$.) When $n \geq 3$ we have by Lefschetz theorem $H^{2}(Q ; \mathbb{R}) \cong \mathbb{R}$, therefore by Moser argument all Kähler forms on $Q$ are symplectically equivalent up to a constant factor. When $n=2, Q \subset \mathbb{C} P^{3}$ is symplectomorphic to $\left(\mathbb{C} P^{1} \times \mathbb{C} P^{1}, \omega_{F S} \oplus \omega_{F S}\right)$. Also note that the symplectic structure on $Q$ (in any dimension) does not depend (up to symplectomorphism) on the specific choice of the defining polynomial $q$ (this follows from Moser argument too since the space of smooth quadrics is connected).

6.3.1 Topology of the quadric The quadric has the following homology:

$$
H_{i}(Q ; \mathbb{Z}) \cong \begin{cases}0 & \text { if } i=\text { odd } \\ \mathbb{Z} & \text { if } i=\text { even } \neq n\end{cases}
$$

Moreover, when $n=$ even, $H_{n}(Q ; \mathbb{Z}) \cong \mathbb{Z} \oplus \mathbb{Z}$. To see the generators of $H_{n}(Q ; \mathbb{Z})$, write $n=2 k$. There exist two families $\mathcal{F}, \mathcal{F}^{\prime}$ of complex $k$-dimensional planes lying in $Q$ (see Griffiths and Harris [34]). Let $P \in \mathcal{F}, P^{\prime} \in \mathcal{F}^{\prime}$ be two such planes belonging to different families. Put $a=[P], b=\left[P^{\prime}\right]$. Then $H_{n}(Q ; \mathbb{Z})=\mathbb{Z} a \oplus \mathbb{Z} b$ and $h^{\bullet k}=a+b$. Moreover, we have:

$$
\begin{aligned}
& \text { for } k=\text { odd : } \quad a \cdot b=[\mathrm{pt}], a \cdot a=b \cdot b=0, \\
& \text { for } k=\text { even : } \quad a \cdot b=0, a \cdot a=b \cdot b=[\mathrm{pt}] .
\end{aligned}
$$


Here and in what follows we have denoted by $\cdot$ the intersection product in singular homology.

6.3.2 Quantum homology of the quadric Let $h \in H_{2 n-2}(Q ; \mathbb{Z})$ be the class of a hyperplane section (coming from the embedding $Q \subset \mathbb{C} P^{n+1}$ ), $p \in H_{0}(Q ; \mathbb{Z})$ the class of a point and $u \in H_{2 n}(Q ; \mathbb{Z})$ the fundamental class. We will first describe the quantum cohomology over $\mathbb{Z}$. Define $\Lambda^{\mathbb{Z}}=\mathbb{Z}\left[t, t^{-1}\right]$ where $\operatorname{deg} t=-N_{L}$. Here $N_{L}$ is the minimal Maslov number of a Lagrangian submanifold that will appear later on. Note that $c_{1}(Q)=n \operatorname{PD}(h)$, hence $N_{L} \mid 2 n$. Let $Q H\left(Q ; \Lambda^{\mathbb{Z}}\right)=H(Q ; \mathbb{Z}) \otimes \Lambda^{\mathbb{Z}}$ be the quantum homology endowed with the quantum product $*$.

Proposition 6.3.1 (See Beauville [6].) The quantum product satisfies the following identities:

$$
\begin{aligned}
h^{* j}=h^{\bullet j} \forall 0 \leq j \leq n-1, & h^{* n}=2 p+2 u t^{2 n / N_{L}}, \quad h^{*(n+1)}=4 h t^{2 n / N_{L}}, \\
& p * p=u t^{4 n / N_{L}} .
\end{aligned}
$$

When $n=$ even we have the following additional identities:

(i) $h * a=h * b$.

(ii) If $n / 2=$ odd then $a * b=p, a * a=b * b=u t^{2 n / N_{L}}$.

(iii) If $n / 2=$ even then $a * a=b * b=p, a * b=u t^{2 n / N_{L}}$.

Proof The first three identities and the fact that $h * a=h * b$ are proved in [6]. To prove the remaining two identities write $n=2 k$. Recall from [6] that

$(a-b) *(a-b)=((a-b) \cdot(a-b)) \frac{1}{2}\left(h^{* n}-4 u t^{2 n / N_{L}}\right)=((a-b) \cdot(a-b))\left(p-u t^{2 n / N_{L}}\right)$ (where $-\cdot-$ is the classical intersection product). Substituting (50) in this we obtain

$$
(a-b) *(a-b)=(-1)^{k} 2\left(p-u t^{2 n / N_{L}}\right) .
$$

On the other hand we have $h^{* k}=h^{\bullet k}=a+b$, hence

$$
(a+b) *(a+b)=h^{* n}=2 p+2 u t^{2 n / N_{L}} .
$$

Next we claim that $a * a=b * b$. Indeed $a * a-b * b=(a+b) *(a-b)=h^{* k} *(a-b)=0$. The desired identities follow from this together with (51), (52). 


\subsubsection{Quantum structures for Lagrangian submanifolds of the quadric The quad-} ric $Q$ has Lagrangian spheres. To see this write $Q$ as $Q=\left\{z_{0}^{2}+\cdots+z_{n}^{2}=z_{n+1}^{2}\right\} \subset$ $\mathbb{C} P^{n+1}$. Then $L=\left\{\left[z_{0}: \cdots: z_{n+1}\right] \in Q \mid z_{i} \in \mathbb{R}, \forall i\right\}$ is a Lagrangian sphere. We assume from now on that $n \geq 2$.

Lemma 6.3.2 Let $L \subset Q$ be a Lagrangian submanifold with $H_{1}(L ; \mathbb{Z})=0$. Then, $N_{L}=2 n, L$ is wide and there is a canonical isomorphism $Q H(L) \cong H\left(L ; \mathbb{Z}_{2}\right) \otimes \Lambda$. Moreover, if we denote by $\alpha_{0} \in Q H_{0}(L)$ the class of a point, by $\alpha_{n} \in Q H_{n}(L)$ the fundamental class and similarly by $p \in Q H_{0}(Q)$ the class of the point and by $u \in \mathrm{QH}_{2 n}(Q)$ the fundamental class, then we have:

(i) $p * \alpha_{0}=\alpha_{0} t, p * \alpha_{n}=\alpha_{n} t$.

(ii) $i_{L}\left(\alpha_{0}\right)=p+u t$.

(iii) If $n$ is even then $\alpha_{0} * \alpha_{0}=\alpha_{n} t$.

Remark 6.3.3 Suppose that $L$ is a monotone Lagrangian which is orientable and relative spin (see Fukaya, Oh, Ohta and Ono [33] for the definition). In that case, it is possible to coherently orient the moduli spaces of pseudo-holomorphic disks with boundary on $L$ using the theory of [33]. It seems very likely that these orientations are compatible with the quantum operations based on our pearly moduli spaces, hence we expect our theory to work over $\mathbb{Z}$. Assuming this, let $L$ be a Lagrangian as in Lemma 6.3.2 and suppose in addition that $L$ is relative spin $\left(H_{1}(L ; \mathbb{Z})=0\right.$ automatically implies orientability). Then we expect the formulae in (i) and (ii) to become:

(i') $p * \alpha_{0}=-\alpha_{0} t, p * \alpha_{n}=-\alpha_{n} t$.

(ii') $i_{L}\left(\alpha_{0}\right)=p-u t$

Proofs of Lemma 6.3.2 and Remark 6.3.3 Following Remark 6.3.3 we will carry out the proof over the ring $\mathbb{K}$ which is either $\mathbb{Z}_{2}$ or $\mathbb{Z}$. In the latter case the proof is not $100 \%$ rigorous in the sense that it depends on the verification that our theory indeed works over $\mathbb{Z}$. We remark that for $\mathbb{K}=\mathbb{Z}_{2}$ the proof below is completely rigorous (and in this case we may also drop the assumptions that $L$ is orientable and relative spin). We will use the ring $\Lambda=\mathbb{K}\left[t^{-1}, t\right]$ with the same grading as before, ie $\operatorname{deg} t=-N_{L}$.

Due to $H_{1}(L ; \mathbb{Z})=0$ and $C_{Q}=n$ we see that $N_{L}=2 n$. By Theorem 1.2.2 we deduce that $L$ is wide. Moreover, by Proposition 4.5.1 there is a canonical isomorphism $Q H_{*}(L) \cong(H(L ; \mathbb{K}) \otimes \Lambda)_{*}$.

We first prove the lemma and the remark under the additional assumption that $n=$ $\operatorname{dim} L \geq 3$. The case $n=2$ will be treated separately at the end of the proof. 
We start with the statement at point (ii'). It easily follows from the definition of the quantum inclusion map that $i_{L}\left(\alpha_{0}\right)=p+e u t$, for some $e \in \mathbb{K}$. Clearly $h * \alpha_{0}=0$ since $h * \alpha_{0}$ belongs to $Q H_{-2}(L) \cong Q H_{2 n-2}(L)=0$ (since $2 n-2>n$ ). Therefore we have

$$
0=i_{L}\left(h * \alpha_{0}\right)=h *(p+e u t)=h * p+e h t .
$$

On the other hand a simple computation based on the identities of Proposition 6.3.1 gives $h * p=h t$. It follows that $e=-1$. This proves point (ii').

We turn to proving point (i'). By Proposition 6.3.1 $p \in Q H_{0}(Q ; \Lambda)$ is an invertible element, hence $p *(-): Q H_{i}(L) \rightarrow Q H_{i-2 n}(L)$ is an isomorphism for every $i$. But $Q H_{0}(L) \cong \mathbb{K} \alpha_{0}$ and $Q H_{-2 n}(L) \cong \mathbb{K} \alpha_{0} t$. Therefore $p * \alpha_{0}=\epsilon \alpha_{0} t$, where $\epsilon= \pm 1$. It remains to determine the precise sign of $\epsilon$. Using the formula in (ii') we obtain

$$
i_{L}\left(p * \alpha_{0}\right)=i_{L}\left(\epsilon \alpha_{0} t\right)=\epsilon\left(p t-u t^{2}\right) .
$$

On the other hand we have

$$
i_{L}\left(p * \alpha_{0}\right)=p * i_{L}\left(\alpha_{0}\right)=p *(p-u t)=u t^{2}-p t .
$$

Comparing this to (53) immediately shows that $\epsilon=-1$. The proof of the identity $p * \alpha_{n}=-\alpha_{n} t$ is similar. This concludes the proof of point (i').

We now turn to the proof in case $n=2$. In this case $Q \approx S^{2} \times S^{2}$ endowed with the split symplectic form $\omega \oplus \omega$ with both $S^{2}$ factors having the same area. Put $a=\left[S^{2} \times \mathrm{pt}\right]$, $b=\left[\mathrm{pt} \times S^{2}\right] \in H_{2}(Q ; \mathbb{Z})$ and denote by $\operatorname{inc}_{*}: H_{*}(L ; \mathbb{Z}) \rightarrow H_{*}(Q ; \mathbb{Z})$ the (classical) map induced by the inclusion $L \subset Q$. Note that $L$ must be a Lagrangian sphere, hence $\int_{L} \omega=0$ and $\operatorname{inc}_{*}([L]) \cdot \operatorname{inc}_{*}([L])=-2$. It follows that inc $*([L])= \pm(a-b)$. Finally, in this dimension the hyperplane class $h$ satisfies $h=a+b$.

As $n=2$ we have $N_{L}=4$ and so $\operatorname{deg} t=-4$. As before, since $p$ is invertible we can write $p * \alpha_{0}=\epsilon \alpha_{0} t$, where $\epsilon= \pm 1$, and $i_{L}\left(\alpha_{0}\right)=p+e u t$ with $e \in \mathbb{Z}$. It follows that

$$
i_{L}\left(p * \alpha_{0}\right)=p *(p+e u t)=u t^{2}+e p t .
$$

On the other hand we also have:

$$
i_{L}\left(p * \alpha_{0}\right)=i_{L}\left(\epsilon \alpha_{0} t\right)=\epsilon t(p+e u t)=\epsilon e u t^{2}+\epsilon p t .
$$

It follows that $\epsilon e=1$, hence $e=\epsilon= \pm 1$. This proves formulas (i) and (ii) over $\mathbb{Z}_{2}$ (that $p * \alpha_{2}= \pm \alpha_{2} t$ follows immediately from the fact that $p$ is invertible).

It remains to determine the sign of $e$ and $\epsilon$, so we now work over $\mathbb{Z}$. For this end write $h * \alpha_{0}=r \alpha_{2}$ with $r \in \mathbb{Z}$. Note that $\alpha_{2}=[L]$ so

$$
i_{L}\left(h * \alpha_{0}\right)=i_{L}\left(r \alpha_{2} t\right)=r \operatorname{inc}_{*}([L]) t= \pm r(a-b) t .
$$


(Here we have used the fact that for the fundamental class $[L]$ we have $i_{L}([L])=$ $\operatorname{inc}_{*}([L])$.) On the other hand

$i_{L}\left(h * \alpha_{0}\right)=h * i_{L}\left(\alpha_{0}\right)=h *(p+e u t)=h t+e h t=(1+e) h t=(1+e)(a+b) t$.

It follows that $(1+e)(a+b) t= \pm r(a-b) t$. This implies $r=1+e=0$, hence $e=-1$. The proof of formulae (i), (i'), (ii), (ii') is now complete for every $n \geq 2$.

Finally, we prove (iii) (only over $\mathbb{Z}_{2}$ ). By Proposition 6.3 .1 when $n=$ even the element $a \in Q H_{n}(Q ; \Lambda)$ is invertible (even if we work with coefficients in $\mathbb{Z}_{2}$ ). Therefore $a * \alpha_{n}=\alpha_{0}$ and $a * \alpha_{0}=\alpha_{n} t$. It follows that

$$
\alpha_{0} * \alpha_{0}=\left(a * \alpha_{n}\right) * \alpha_{0}=a *\left(\alpha_{n} * \alpha_{0}\right)=a * \alpha_{0}=\alpha_{n} t .
$$

The following result shows that for $n=$ even, at least homologically, spheres are the only type of Lagrangian in $Q$ with $H_{1}(L ; \mathbb{Z})=0$.

Theorem 6.3.4 Assume $n=\operatorname{dim}_{\mathbb{C}} Q=$ even. Let $L \subset Q$ be a Lagrangian submanifold with $H_{1}(L ; \mathbb{Z})=0$. Then $H_{*}\left(L ; \mathbb{Z}_{2}\right) \cong H_{*}\left(S^{n} ; \mathbb{Z}_{2}\right)$.

Proof In view of the isomorphism $Q H_{*}(L) \cong\left(H\left(L ; \mathbb{Z}_{2}\right) \otimes \Lambda\right)_{*}$, for every $q \in \mathbb{Z}$, $0 \leq r<2 n$ we have

$$
Q H_{2 n q+r}(L) \cong \begin{cases}H_{r}\left(L ; \mathbb{Z}_{2}\right) & \text { if } 0 \leq r \leq n \\ 0 & \text { if } n+1 \leq r \leq 2 n-1\end{cases}
$$

Reducing modulo 2 the identities from Proposition 6.3.1 it follows that $a \in Q H_{n}(Q ; \Lambda)$ is an invertible element. Thus $a *(-): Q H_{i}(L) \rightarrow Q H_{i-n}(L)$ is an isomorphism for every $i \in \mathbb{Z}$. It now easily follows from $(54)$ that $H_{i}\left(L ; \mathbb{Z}_{2}\right)=0$ for every $0<i<n$. $\square$

We are not aware of the existence of a Lagrangian submanifold in $Q$ with $H_{1}(L ; \mathbb{Z})=0$ which is not diffeomorphic to a sphere, and it is tempting to conjecture that spheres are indeed the only examples.

Remark 6.3.5 Theorem 6.3.4 can be also proved by Seidel's method of graded Lagrangian submanifolds [51]. Indeed for $n$ even the quadric has a Hamiltonian $S^{1}$-action which induces a shift by $n$ on $Q H_{*}(L)$. To see this write $n=2 k$ and write $Q$ as $Q=\left\{\sum_{j=0}^{k} z_{j} z_{j+1+k}=0\right\}$. Then $S^{1}$ acts by sending $t \cdot\left[z_{0}: \cdots: z_{2 k+1}\right]$ to $\left[t z_{0}: \cdots: t z_{k}: z_{k+1}: \cdots: z_{2 k+1}\right]$. A simple computation of the weights of the action at a fixed point gives a shift of $n$ on graded Lagrangian submanifolds in the sense of [51]. When $n=$ odd our methods (as well as those of [51]) do not seem to yield a result similar to Theorem 6.3.4. However the works of Buhovsky [17] and of Seidel [52] may provide evidence that such a result should hold. 
Denote $\mathcal{J}$ the space of almost complex structures compatible with the symplectic structure of $Q$. The next result is a straightforward consequence of Lemma 6.3.2 and it concludes the proofs of the properties claimed in Theorem 2.3.4 and Corollary 1.2.13.

Lemma 6.3.6 Let $L \subset Q$ be a Lagrangian submanifold with $H_{1}(L ; \mathbb{Z})=0$. Assume $n=\operatorname{dim}_{\mathbb{C}} Q \geq 2$. Then the following holds:

(i) Let $x \in L$ and $z \in Q \backslash L$. Then for every $J \in \mathcal{J}$ there exists a $J$-holomorphic disk $u:(D, \partial D) \rightarrow(Q, L)$ with $u(-1)=x, u(0)=z$ and $\mu([u])=2 n$.

(ii) Assume that $n=$ even. Let $x^{\prime}, x^{\prime \prime}, x^{\prime \prime \prime} \in L$. Then for every $J \in \mathcal{J}$ there exists a $J$-holomorphic disk $u:(D, \partial D) \rightarrow(Q, L)$ with $u\left(e^{2 \pi i / 3}\right)=x^{\prime}, u(1)=x^{\prime \prime}$, $u\left(e^{4 \pi i / 3}\right)=x^{\prime \prime \prime}$ and $\mu([u])=2 n$.

Proof The first point follows as usual by considering a Morse function $f: L \rightarrow \mathbb{R}$ with a single maximum and a single minimum as well as a perfect Morse function $h: Q \rightarrow \mathbb{R}$. We let the minimum of $h$ be denoted by $p$ (by a slight abuse in notation we identify the critical points of $h$ and the corresponding singular homology classes) and we denote the minimum of $f$ by $m$ and its maximum by $w$. As $L$ is wide both $m$ and $w$ are cycles in the associated pearl complex.

Point (i) in Lemma 6.3.2 gives, at the chain level, $p * m=m t$ which implies the first point of our lemma. The second point is proved by considering a second Morse function $f^{\prime}: L \rightarrow \mathbb{R}$ with a unique minimum $m^{\prime}$. Relation (iii) in Lemma 6.3.2 now gives (on the chain level) $m * m^{\prime}=w t$, which proves the needed statement.

\subsection{Narrow Lagrangians in $\mathbb{C P}^{n}$}

The purpose of this section is to construct the monotone narrow Lagrangians mentioned in Example 1.2.10. The construction is based on the decomposition technique developed in [7] and on the Lagrangian circle bundle construction from [8].

Let $\left(M^{2 n}, \omega\right)$ be a symplectic manifold for which $[\omega] \in H^{2}(M ; \mathbb{R})$ admits an integral lift in $H^{2}(M ; \mathbb{Z})$. Fix such a lift $a_{\omega}$. Let $\Sigma^{2 n-2} \subset M^{2 n}$ be a symplectic hyperplane section in the sense that $\Sigma$ is a symplectic submanifold whose homology class is dual to a positive multiple of $a_{\omega}$, ie $\operatorname{PD}[\Sigma]=k a_{\omega} \in H^{2}(M ; \mathbb{Z})$ for some integer $k>0$. By rescaling $\omega$ we will assume from now on, without loss of generality, that $k=1$.

Assume further that $M$ is a complex manifold, that $\omega$ is a Kähler form and that $\Sigma \subset M$ is a complex submanifold (so that $\Sigma \subset M$ is a smooth ample divisor). Put $\omega_{\Sigma}=\left.\omega\right|_{\Sigma}$ and $a_{\Sigma}=\left.a_{\omega}\right|_{\Sigma} \in H^{2}(\Sigma ; \mathbb{Z})$. Let $\pi: P \rightarrow \Sigma$ be a circle bundle with Euler class $a_{\Sigma}$ and $\alpha$ a connection $1-$ form on $P$ normalized so that $d \alpha=-\pi^{*} \omega_{\Sigma}$. 
Denote by $E_{\Sigma} \rightarrow \Sigma$ the associated unit disk bundle, $E_{\Sigma}=(P \times[0,1)) / \sim$, where $\left(p^{\prime}, 0\right) \sim\left(p^{\prime \prime}, 0\right)$ if and only if $\pi\left(p^{\prime}\right)=\pi\left(p^{\prime \prime}\right)$. We endow $E_{\Sigma}$ with the following symplectic structure: $\omega_{\text {can }}=\pi^{*} \omega_{\Sigma}+d\left(r^{2} \alpha\right)$, where $r$ is the second coordinate on $P \times[0,1)$. Note that with our normalization $\left.\omega_{\text {can }}\right|_{\Sigma}=\omega_{\Sigma}$ and the area of each fibre of $E_{\Sigma}$ with respect to $\omega_{\text {can }}$ is 1 .

By the results of [7] there exists a compact isotropic CW-complex $\Delta \subset M \backslash \Sigma$ and a symplectomorphism $F:\left(E_{\Sigma}, \omega_{\text {can }}\right) \longrightarrow(M \backslash \Delta, \omega)$. Moreover, for every $x \in \Sigma \subset E_{\Sigma}$ we have $F(x)=x$. In most cases $\Delta$ is a Lagrangian CW-complex, ie $\operatorname{dim} \Delta=$ $\frac{1}{2} \operatorname{dim} M-$ this is called the critical case. In special situations it may happen that $\operatorname{dim} \Delta<\frac{1}{2} \operatorname{dim} M$, which we call the subcritical case. The dimension of $\Delta$ is in fact determined by the critical points of a plurisubharmonic function $\varphi: M \backslash \Sigma \rightarrow \mathbb{R}$ canonically determined by $\Sigma$ and the complex structure of $M$. The CW-complex $\Delta$ is called the isotropic (or sometimes Lagrangian) skeleton. We refer the reader to Biran [7] for more details on this type of decompositions. See also Eliashberg and Gromov [28] and Eliashberg [27] for the foundations of symplectic geometry of Stein manifolds, as well as Biran and Cieliebak [10;9] and Biran [8] for applications of these concepts to questions on Lagrangian submanifolds. We will identify from now on $(M \backslash \Delta, \omega)$ with $\left(E_{\Sigma}, \omega_{\text {can }}\right)$ via the map $F$.

Let $L \subset\left(\Sigma, \omega_{\Sigma}\right)$ be a Lagrangian submanifold. Fix $0<r_{0}<1$. Put

$$
\Gamma_{L}=\pi^{-1}(L) \times\left\{r_{0}\right\} \subset E_{\Sigma} \approx M \backslash \Delta .
$$

Note that $\pi: \Gamma_{L} \rightarrow L$ is a circle bundle isomorphic to the restriction of $P \rightarrow \Sigma$ to $L$. A simple computation shows that $\Gamma_{L}$ is Lagrangian with respect to $\omega$. We will view $\Gamma_{L}$ as a Lagrangian submanifold of $M$, but it is important to note that $\Gamma_{L}$ is disjoint from $\Delta$. We remark also that $\Gamma_{L}$ depends on the value of $r_{0}$. In fact, different values of $r_{0}$ give rise to Lagrangians $\Gamma_{L}$ with different area classes. Below we will make a specific choice of $r_{0}$ and call $\Gamma_{L}$ the Lagrangian circle bundle over $L$. We refer the reader to [8] for more details on the subject.

Suppose now that $L \subset \Sigma$ is monotone with proportionality constant $\eta=\omega / \mu$.

Proposition 6.4.1 Assume that $\operatorname{dim} M \geq 6$, or that $\operatorname{dim} M=4$ and $\Delta$ is subcritical. Let $r_{0}^{2}=2 \eta /(2 \eta+1)$. Then the Lagrangian $\Gamma_{L} \subset M$ is monotone. It has minimal Maslov number $N_{\Gamma_{L}}=2$ and proportionality constant $\hat{\eta}=\eta /(2 \eta+1)$.

Proof Fix $A \in \pi_{2}\left(M, \Gamma_{L}\right)$ and let $u:(D, \partial D) \rightarrow\left(M, \Gamma_{L}\right)$ be a representative of $A$. As $\operatorname{dim} \Delta+2<\operatorname{dim} M$ we may assume by transversality that the image of $u$ is disjoint from $\Delta$, hence lies in $E_{\Sigma}$. Denote by $x_{1}, \ldots, x_{k}$ the intersection points of $u$ with $\Sigma$ and assume that they are all transverse. Moreover, we may assume 
that each $x_{i}$ corresponds to a single interior point $z_{i} \in D$, so that $u^{-1}\left(x_{i}\right)=\left\{z_{i}\right\}$. After a suitable homotopy of $u($ rel $\partial D)$ we may assume that the points $x_{i}$ all lie in $L$. Denote by $D_{x_{i}} \subset E_{\Sigma}$ the disk of radius $r_{0}$ lying in the fibre over $x_{i}$ (ie $\left.D_{x_{i}}=\left(\pi^{-1}\left(x_{i}\right) \times\left[0, r_{0}\right]\right) / \sim\right)$. Note that the boundary of $D_{x_{i}}$ lies in $\Gamma_{L}$. After a further homotopy of $u$ we may assume that there exist small disks $B_{i} \subset D$ around each $z_{i}$ such that $u$ maps each $B_{i}$ to $\pm D_{x_{i}}$. Here, \pm stands for the two possible orientations on $D_{x_{i}}$, according to whether $\left.u\right|_{B_{i}}: B_{i} \rightarrow D_{x_{i}}$ preserves or reverses orientation. Put $S=D \backslash\left(\bigcup_{i=1}^{k}\right.$ Int $\left.B_{i}\right)$. Put $v=\left.u\right|_{S}$. Clearly the image of $v$ is disjoint from $\Sigma$ and moreover $v$ maps the boundary of $S$ to $\Gamma_{L}$. After another homotopy of $v$, rel $\partial S$ we may also assume that the image of $v$ lies in $P \times\left\{r_{0}\right\} \subset E_{\Sigma}$. Note that

$$
\left.\omega_{\mathrm{can}}\right|_{P \times\left\{r_{0}\right\}}=\left.\left(\pi^{*} \omega_{\Sigma}+2 r d r \wedge \alpha+r^{2} d \alpha\right)\right|_{P \times\left\{r_{0}\right\}}=\left(1-r_{0}^{2}\right) \pi^{*} \omega_{\Sigma},
$$

hence we have

$$
\int_{S} v^{*} \omega=\left(1-r_{0}^{2}\right) \int_{S}(\pi \circ v)^{*} \omega_{\Sigma}
$$

Denote by $\epsilon_{i} \in\{-1,1\}$ the intersection index of $\left.u\right|_{B_{i}}$ with $\Sigma$. We have

$$
\begin{aligned}
\omega(A) & =\int_{D} u^{*} \omega=\sum_{i=1}^{k} \int_{B_{i}} u^{*} \omega+\int_{S} v^{*} \omega \\
& =\left(\sum_{i=1}^{k} \epsilon_{i}\right) r_{0}^{2}+\left(1-r_{0}^{2}\right) \int_{S}(\pi \circ v)^{*} \omega_{\Sigma}, \\
\mu(A) & =\sum_{i=1}^{k} \mu\left(\left[\left.u\right|_{B_{i}}\right]\right)+\mu([v])=2\left(\sum_{i=1}^{k} \epsilon_{i}\right)+\mu([v]) .
\end{aligned}
$$

Denote by $\mu_{L}: H_{2}(\Sigma, L) \rightarrow \mathbb{Z}$ the Maslov index of $L \subset \Sigma$. A simple computation shows that $\mu([v])=\mu_{L}([\pi \circ v]$ ) (see Proposition 4.1.A in [8] and its proof.) Next, note that $[\pi \circ v]$ in fact lies in the image of $\pi_{2}(\Sigma, L) \rightarrow H_{2}(\Sigma, L)$. By the monotonicity of $L$ we now get: $\int_{S}(\pi \circ v)^{*} \omega_{\Sigma}=\eta \mu_{L}([\pi \circ v])$. Using this and (55) we deduce that $\Gamma_{L} \subset M$ will be monotone if $r_{0}^{2} / 2=\left(1-r_{0}^{2}\right) \eta$. Solving this equation gives $r_{0}^{2}=2 \eta /(2 \eta+1)$.

Remark The Lagrangian $\Gamma_{L}$, when viewed as a submanifold of $M \backslash \Sigma$, is obviously monotone too (in fact, for every value of $r_{0}$ ). Its minimal Maslov number (as a Lagrangian in $M \backslash \Sigma), N_{\Gamma_{L}}^{\prime}$, satisfies $N_{\Gamma_{L}}^{\prime}=N_{L}$. See Biran [8] for more details.

Based on the above we can construct examples of narrow Lagrangians in $\mathbb{C} P^{n}$. 
6.4.1 Narrow Lagrangians in $\mathbb{C} P^{n}$ Consider $M=\mathbb{C} P^{n}, n \geq 3$, endowed with the following normalization of the standard symplectic structure $\omega_{\mathrm{FS}}^{\prime}=2 \omega_{\mathrm{FS}}$. (The normalization here is made so that $\left[\omega_{\mathrm{FS}}^{\prime}\right] \in H^{2}\left(\mathbb{C} P^{n} ; \mathbb{Z}\right)$ is 2 times the generator.) Let $\Sigma=Q^{2 n-2} \subset \mathbb{C} P^{n}$ be the smooth complex quadric hypersurface, given for example by $Q=\left\{z_{0}^{2}+\cdots+z_{n}^{2}=0\right\}$. The Lagrangian skeleton in this case is $\Delta=\mathbb{R} P^{n}=\left\{\left[z_{0}: \cdots: z_{n}\right] \mid z_{i} \in \mathbb{R}, \forall i\right\}$. See Biran [7] for the computation.

Let $L \subset Q^{2 n-2}$ be any monotone Lagrangian (eg a Lagrangian sphere), and consider $\Gamma_{L} \subset \mathbb{C} P^{n}$ constructed as above. By construction, $\Gamma_{L} \cap \mathbb{R} P^{n}=\varnothing$. By Corollary 1.2.11 $\mathbb{R} P^{n}$ is wide. It follows from Corollary 1.2.8 that $\Gamma_{L}$ is narrow.

The same construction actually works also for $M=\mathbb{C} P^{2}$, although $\Delta$ is not subcritical. In this case $Q \approx S^{2}$ and we can take $L \subset S^{2}$ to be a circle which divides $S^{2}$ into two disks of equal areas. The corresponding Lagrangian circle bundle $\Gamma_{L}$ is a 2-dimensional torus in $\mathbb{C} P^{2}$. The fact that $\Gamma_{L}$ is monotone follows from a direct computation of Maslov indices and areas for each of the three generators of $\pi_{2}\left(\mathbb{C} P^{2}, L\right) \cong \mathbb{Z}^{\oplus 3}$. Thus we obtain a narrow Lagrangian torus $\Gamma_{L} \subset \mathbb{C} P^{2}$. We remark that $\Gamma_{L}$ is not symplectically equivalent to the Clifford torus $\mathbb{T}_{\text {clif }}^{2} \subset \mathbb{C} P^{2}$ since the latter is wide. On the other hand, these two tori, $\mathbb{T}_{\text {clif }}$ and $\Gamma_{L}$ turn out to be Lagrangian isotopic one to the other. It would be interesting to understand the relation of this example with Chekanov's exotic torus [18] as well as with the works Eliashberg and Polterovich [29] and Blechman and Polterovich [14].

6.4.2 More examples One can iterate the Lagrangian circle bundle construction by looking at hyperplane sections of hyperplane sections $\Sigma^{\prime} \subset \Sigma \subset M$ etc. (with different choices of $\Sigma$ 's as well as different choices of $L$ 's) and obtain many examples of narrow monotone tori in $\mathbb{C} P^{n}$. It would be interesting to figure out how many of them are symplectically nonequivalent. It would also be interesting to understand the relation of these tori to the recent series of pairwise nonequivalent Lagrangian tori constructed by Chekanov and Schlenk [19].

\section{Open questions}

Traditionally, the class of monotone Lagrangians has been of interest because it provides a context in which Floer homology remains reasonably simple to define and, simultaneously, is sufficiently rich so as to provide a wide variety of examples. However, the structural rigidity properties discussed in this paper indicate that this class is also interesting in itself. We remark that wide monotone Lagrangians also satisfy a form of numerical (or arithmetic) rigidity (some results on this can be found in [12]). 
Of course, many questions remain open at this time. An obvious issue is whether higher order operations - beyond the module and product structures, in particular - can be used to produce further extensions of the results proved here. A considerable amount of additional technical complications are involved in setting up the machinery needed to deal with that degree of generality so we have not pursued this avenue here. In a different direction, it is clearly possible to further pursue relative packing computations as well as various Gromov radius estimates.

Another obvious problem is to establish the theory described here with coefficients in $\mathbb{Z}$. As already mentioned in Section 6.3.3 Remark 6.3.3, we expect our theory to work over $\mathbb{Z}$ however we have not rigorously checked the needed compatibility with orientations. Still, it is instructive to see an example showing that this issue is important for certain applications.

Let $Q \subset \mathbb{C} P^{n+1}$ be a smooth complex quadric hypersurface endowed with the symplectic structure induced from $\mathbb{C} P^{n+1}$. The following corollary shows that the composition $j_{L_{1}} \circ i_{L_{0}}$ introduced in Section 2.4 does not vanish for a class of Lagrangians in the quadric, provided that we work with $\mathbb{Z}$ (rather than $\mathbb{Z}_{2}$ ) as the ground ring of coefficients and so - by Theorem 2.4.1 (again with $\mathbb{Z}$-coefficients) - any two Lagrangians in this class intersect. We mark the Corollary with a $*$ to indicate that its proof is not $100 \%$ rigorous.

Corollary* 7.0.2 Let $L_{0}, L_{1} \subset Q$ be two Lagrangians with $H_{1}\left(L_{i} ; \mathbb{Z}\right)=0, i=0,1$ and assume in addition that $L_{0}, L_{1}$ are relative spin (see Fukaya et al [33] for the definition). (For example, $L_{0}$ and $L_{1}$ are two Lagrangian spheres). Then, over $\mathbb{Z}$, the composition $j_{L_{1}} \circ i_{L_{0}}$ does not vanish. In particular $L_{0} \cap L_{1} \neq \varnothing$.

Proof* As $H_{1}\left(L_{i} ; \mathbb{Z}\right)=0$, the Lagrangians $L_{0}, L_{1}$ are orientable, hence in view of the relative spin condition we can orient all the moduli spaces of disks following [33]. The condition $H_{1}\left(L_{i} ; \mathbb{Z}\right)=0$ implies that $N_{L_{0}}=N_{L_{1}}=2 C_{Q}=2 n$. Therefore in the ring $\Lambda_{0,1}$ (from Section 2.4) we have $t_{0}=t_{1}$ or in other words $\Lambda_{0,1} \cong \Lambda_{0} \cong$ $\Lambda_{1} \cong \mathbb{Z}\left[t^{-1}, t\right]$, with $\operatorname{deg} t=-2 n$. (Note again, we are using $\mathbb{Z}$ as the ground ring.)

We will now use the notation from Section 6.3.3, Lemma 6.3.2 and Remark 6.3.3. Recall that by this Lemma and this Remark we have $i_{L_{0}}\left(\alpha_{0}\right)=p-u t$, where $\alpha_{0} \in Q H_{0}\left(L_{0}\right)$ is the generator, $p \in Q H_{0}(Q)$ is the class of a point and $u \in Q H_{2 n}(Q)$ is the fundamental class. Denoting by $\alpha_{n}^{\prime} \in Q H_{n}\left(L_{1}\right)$ the fundamental class we now have by the same lemma and remark (now applied to $L_{1}$ ),

$$
j_{L_{1}} \circ i_{L_{0}}\left(\alpha_{0}\right)=(p-u t) * \alpha_{n}^{\prime}=-\alpha_{n}^{\prime} t-\alpha_{n}^{\prime} t=-2 \alpha_{n}^{\prime} t \neq 0 .
$$

By Theorem 2.4.1, $L_{0} \cap L_{1} \neq \varnothing$. 
We conclude with two conjectures which, we believe, have a significant structural significance for the understanding of the subject so that we want to make them explicit here. We recall that here, as all along the paper, we include in the definition of a monotone Lagrangian submanifold the condition $N_{L} \geq 2$.

Conjecture 1 Any monotone Lagrangian submanifold is either narrow or wide.

Conjecture 2 In a point invertible manifold, if two monotone Lagrangian submanifolds do not intersect, then at least one of them is narrow.

Remark (a) As shown in Theorem 1.2.2 the dichotomy narrow-wide can be established in many relevant cases and we can prove it in a few more. It is true, for example, for $n=\operatorname{dim} L \leq 3$ (at least when $L$ admits a perfect Morse function).

There is an equivalent statement of the conjecture which is worth indicating here. Recall the map $p_{*}: Q^{+} H(L) \rightarrow Q H(L)$ induced by the change of coefficients $\Lambda^{+} \rightarrow \Lambda$ and that we denote by $I Q^{+}(L)$ its image. It is easy to see that the kernel of $p_{*}$ consists precisely of the torsion ideal $T^{+}(L)$ of $Q^{+} H(L)$,

$$
T^{+}(L)=\left\{z \in Q^{+} H(L): \exists m \in \mathbb{N}, t^{m} z=0\right\} .
$$

It is a simple exercise to see that $L$ is wide if and only if $T^{+}(L)=0$ and $L$ is narrow if and only if $T^{+}(L)=Q^{+} H(L)$. Thus the wide-narrow conjecture is equivalent to showing that the torsion ideal of any monotone Lagrangian can only be 0 or coincide with the entire ring.

(b) The difficulty in proving the second conjecture is caused by the following phenomenon (see also Theorem 2.4.1). First, notice that the result immediately follows if one can show that there is a constant $C$ and a class $\alpha \in Q H(M ; \Lambda)$ (with $M$ the ambient symplectic manifold) so that for any monotone, non-narrow Lagrangian $L \subset M$ and any $\phi \in \widetilde{\operatorname{Ham}}(M)$ one has

$$
\operatorname{depth}_{L}(\phi)-C \leq \sigma(\alpha, \phi) \leq \operatorname{height}_{L}(\phi)+C .
$$

By Lemma 5.3.1 (i), if $\alpha$ is invertible (for example, $\alpha=[\mathrm{pt}]$ for a point invertible manifold) the left inequality (56) follows because $\alpha$ acts nontrivially on $Q H(L)$. The second inequality is implied by the second point of the same Lemma if one can show $\alpha \in \operatorname{Im}\left(i_{L}\right)$. Finding a class $\alpha$ which satisfies both properties is however quite nontrivial. Notice that in a point invertible manifold of order $k$ not only is the left inequality in (56) satisfied for $\alpha=$ pt but we can also deduce the estimate $\sigma^{*}\left(\left[\omega^{n}\right], \phi\right):=\inf \left\{\sigma\left([M]+s^{-1} x, \phi\right) \mid x \in Q H(M ; \Lambda)\right\} \leq$ height $_{L}(\phi)+k$ where $x \in Q^{+} H(M), s$ is the Novikov variable in $\Gamma=\mathbb{Z}_{2}\left[s^{-1}, s\right]$, and $\sigma^{*}\left(\omega^{n}, \phi\right)$ is by 
definition the infimum given above (this notation is justified because it coincides with the cohomological spectral invariant of the class $\left.\left[\omega^{n}\right]\right)$. It is clear from the "triangle inequality" that $\sigma([M], \phi) \geq \sigma([\mathrm{pt}], \phi)$ but it is in general not easy to show that $\sigma^{*}\left(\left[\omega^{n}\right], \phi\right) \geq \sigma([\mathrm{pt}], \phi)$.

\section{References}

[1] P Albers, A Lagrangian Piunikhin-Salamon-Schwarz morphism and two comparison homomorphisms in Floer homology arXiv:math/0512037

[2] P Albers, On the extrinsic topology of Lagrangian submanifolds, Int. Math. Res. Not. (2005) 2341-2371 MR2180810

[3] M Audin, F Lalonde, L Polterovich, Symplectic rigidity: Lagrangian submanifolds, from: "Holomorphic curves in symplectic geometry", (M Audin, J Lafontaine, editors), Progr. Math. 117, Birkhäuser, Basel (1994) 271-321 MR1274934

[4] J-F Barraud, O Cornea, Homotopic dynamics in symplectic topology, from: "Morse theoretic methods in nonlinear analysis and in symplectic topology", (P Biran, O Cornea, F Lalonde, editors), NATO Sci. Ser. II Math. Phys. Chem. 217, Springer, Dordrecht (2006) 109-148 MR2276950

[5] J-F Barraud, O Cornea, Lagrangian intersections and the Serre spectral sequence, Ann. of Math. (2) 166 (2007) 657-722 MR2373371

[6] A Beauville, Quantum cohomology of complete intersections, Mat. Fiz. Anal. Geom. 2 (1995) 384-398 MR1484335

[7] P Biran, Lagrangian barriers and symplectic embeddings, Geom. Funct. Anal. 11 (2001) 407-464 MR1844078

[8] P Biran, Lagrangian non-intersections, Geom. Funct. Anal. 16 (2006) 279-326 MR2231465

[9] P Biran, K Cieliebak, Symplectic topology on subcritical manifolds, Comment. Math. Helv. 76 (2001) 712-753 MR1881704

[10] P Biran, K Cieliebak, Lagrangian embeddings into subcritical Stein manifolds, Israel J. Math. 127 (2002) 221-244 MR1900700

[11] P Biran, O Cornea, in preparation

[12] P Biran, O Cornea, Quantum structures for Lagrangian submanifolds arXiv: 0708.4221

[13] P Biran, O Cornea, A Lagrangian quantum homology, from: "New perspectives and challenges in symplectic field theory", (M Abreu, F Lalonde, L Polterovich, editors), CRM Proc. Lecture Notes, Amer. Math. Soc. (2009) to appear

[14] L Blechman, L Polterovich, private communication 
[15] L Buhovsky, Multiplicative structures in Lagrangian Floer homology arXiv: math.SG/0608063

[16] L Buhovsky, One explicit construction of a relative packing arXiv:0803.2774v3

[17] L Buhovsky, Homology of Lagrangian submanifolds in cotangent bundles, Israel J. Math. 143 (2004) 181-187 MR2106982

[18] Y V Chekanov, Lagrangian tori in a symplectic vector space and global symplectomorphisms, Math. Z. 223 (1996) 547-559 MR1421954

[19] Y V Chekanov, F Schlenk, Monotone Lagrangian tori in $\mathbb{R}^{2 n}, \mathbb{C} P^{n}$ and products of spheres, in preparation

[20] R Chiang, New Lagrangian submanifolds of $\mathbb{C P}^{n}$, Int. Math. Res. Not. (2004) 24372441 MR2076100

[21] C-H Cho, Holomorphic discs, spin structures, and Floer cohomology of the Clifford torus, Int. Math. Res. Not. (2004) 1803-1843 MR2057871

[22] C-H Cho, Products of Floer cohomology of torus fibers in toric Fano manifolds, Comm. Math. Phys. 260 (2005) 613-640 MR2183959

[23] C-H Cho, Y-G Oh, Floer cohomology and disc instantons of Lagrangian torus fibers in Fano toric manifolds, Asian J. Math. 10 (2006) 773-814 MR2282365

[24] O Cornea, F Lalonde, Cluster homology arXiv:math/0508345

[25] O Cornea, F Lalonde, Cluster homology: an overview of the construction and results, Electron. Res. Announc. Amer. Math. Soc. 12 (2006) 1-12 MR2200949

[26] O Cornea, A Ranicki, Rigidity and gluing for Morse and Novikov complexes, J. Eur. Math. Soc. (JEMS) 5 (2003) 343-394 MR2017851

[27] Y Eliashberg, Topological characterization of Stein manifolds of dimension > 2, Internat. J. Math. 1 (1990) 29-46 MR1044658

[28] Y Eliashberg, M Gromov, Convex symplectic manifolds, from: "Several complex variables and complex geometry, Part 2 (Santa Cruz, CA, 1989)”, (E Bedford, J P D’Angelo, R E Greene, S G Krantz, editors), Proc. Sympos. Pure Math. 52, Amer. Math. Soc. (1991) 135-162 MR1128541

[29] Y Eliashberg, L Polterovich, The problem of Lagrangian knots in four-manifolds, from: "Geometric topology (Athens, GA, 1993)", (W H Kazez, editor), AMS/IP Stud. Adv. Math. 2, Amer. Math. Soc. (1997) 313-327 MR1470735

[30] M Entov, L Polterovich, Rigid subsets of symplectic manifolds, Compos. Math. 145 (2009) 773-826 MR2507748

[31] U Frauenfelder, Gromov convergence of pseudoholomorphic disks, J. Fixed Point Theory Appl. 3 (2008) 215-271 MR2434448 
[32] K Fukaya, Morse homotopy and its quantization, from: "Geometric topology (Athens, GA, 1993)", (W H Kazez, editor), AMS/IP Stud. Adv. Math. 2, Amer. Math. Soc. (1997) 409-440 MR1470740

[33] K Fukaya, Y-G Oh, H Ohta, K Ono, Lagrangian intersection Floer theory - anomaly and obstruction, Preprint

[34] P Griffiths, J Harris, Principles of algebraic geometry, Pure and Applied Math., Wiley-Interscience, New York (1978) MR507725

[35] M Gromov, Pseudoholomorphic curves in symplectic manifolds, Invent. Math. 82 (1985) 307-347 MR809718

[36] D Kwon, Y-G Oh, Structure of the image of (pseudo)-holomorphic discs with totally real boundary condition, Comm. Anal. Geom. 8 (2000) 31-82 MR1730896 Appendix 1 by J-P Rosay

[37] L Lazzarini, Relative frames on J-holomorphic curves, to appear in J. Fixed Point Theory Appl. Available at http://www.math.jussieu.fr/ lazzarin/ articles.html

[38] L Lazzarini, Existence of a somewhere injective pseudo-holomorphic disc, Geom. Funct. Anal. 10 (2000) 829-862 MR1791142

[39] D McDuff, Hamiltonian $S^{1}$-manifolds are uniruled arXiv:0706.0675v2

[40] D McDuff, D Salamon, J-holomorphic curves and symplectic topology, Amer. Math. Soc. Colloq. Publ. 52, Amer. Math. Soc. (2004) MR2045629

[41] Y-G Oh, Mini-max theory, spectral invariants and geometry of the Hamiltonian diffeomorphism group arXiv:math/0206092

[42] Y-G Oh, Floer cohomology of Lagrangian intersections and pseudo-holomorphic disks. I, Comm. Pure Appl. Math. 46 (1993) 949-993 MR1223659

[43] Y-G Oh, Floer cohomology, spectral sequences, and the Maslov class of Lagrangian embeddings, Internat. Math. Res. Notices (1996) 305-346 MR1389956

[44] Y-G Oh, Relative Floer and quantum cohomology and the symplectic topology of Lagrangian submanifolds, from: "Contact and symplectic geometry (Cambridge, 1994)", (C B Thomas, editor), Publ. Newton Inst. 8, Cambridge Univ. Press (1996) 201-267 MR1432465

[45] Y-G Oh, Construction of spectral invariants of Hamiltonian paths on closed symplectic manifolds, from: "The breadth of symplectic and Poisson geometry", (J E Marsden, T S Ratiu, editors), Progr. Math. 232, Birkhäuser, Boston (2005) 525-570 MR2103018

[46] Y-G Oh, Spectral invariants, analysis of the Floer moduli space, and geometry of the Hamiltonian diffeomorphism group, Duke Math. J. 130 (2005) 199-295 MR2181090 
[47] Y-G Oh, Lectures on Floer theory and spectral invariants of Hamiltonian flows, from: "Morse theoretic methods in nonlinear analysis and in symplectic topology", (P Biran, O Cornea, F Lalonde, editors), NATO Sci. Ser. II Math. Phys. Chem. 217, Springer, Dordrecht (2006) 321-416 MR2276955

[48] S Piunikhin, D Salamon, M Schwarz, Symplectic Floer-Donaldson theory and quantum cohomology, from: "Contact and symplectic geometry (Cambridge, 1994)", (C B Thomas, editor), Publ. Newton Inst. 8, Cambridge Univ. Press (1996) 171-200 MR1432464

[49] M Schwarz, On the action spectrum for closed symplectically aspherical manifolds, Pacific J. Math. 193 (2000) 419-461 MR1755825

[50] P Seidel, $\pi_{1}$ of symplectic automorphism groups and invertibles in quantum homology rings, Geom. Funct. Anal. 7 (1997) 1046-1095 MR1487754

[51] P Seidel, Graded Lagrangian submanifolds, Bull. Soc. Math. France 128 (2000) 103149 MR1765826

[52] P Seidel, Exact Lagrangian submanifolds in $T^{*} S^{n}$ and the graded Kronecker quiver, from: "Different faces of geometry", (S Donaldson, Y Eliashberg, M Gromov, editors), Int. Math. Ser. 3, Kluwer/Plenum, New York (2004) 349-364 MR2103000

[53] C Viterbo, Symplectic topology as the geometry of generating functions, Math. Ann. 292 (1992) 685-710 MR1157321

School of Mathematical Sciences, Tel-Aviv University

Ramat-Aviv, Tel-Aviv 69978, Israel

Department of Mathematics and Statistics, University of Montreal

C.P. 6128 Succ. Centre-Ville Montreal, QC H3C 3J7, Canada

biran@math.tau.ac.il, cornea@dms.umontreal.ca

Proposed: Leonid Polterovich

Seconded: Yasha Eliashberg, Danny Calegari
Received: 5 November 2008

Revised: 22 July 2009 